\title{
Exocomets: A spectroscopic survey ${ }^{\star}$
}

I. Rebollido ${ }^{1}$, C. Eiroa ${ }^{1,2}$, B. Montesinos ${ }^{3}$, J. Maldonado ${ }^{4}$, E. Villaver ${ }^{1}$, O. Absil ${ }^{5}$, A. Bayo ${ }^{6,7}$, H. Canovas ${ }^{8}$, A. Carmona ${ }^{9}$, Ch. Chen $^{10}$, S. Ertel ${ }^{11}$, Th. Henning ${ }^{12}$, D. P. Iglesias ${ }^{6,7}$, R. Launhardt ${ }^{12}$, R. Liseau ${ }^{13}$, G. Meeus ${ }^{1}$, A. Moór ${ }^{14,15}$, A. Mora ${ }^{16}$, J. Olofsson ${ }^{6,7}$, G. Rauw ${ }^{5}$, and P. Riviere-Marichalar ${ }^{17}$

1 Dpto. Física Teórica, Universidad Autónoma de Madrid, Cantoblanco 28049 Madrid, Spain e-mail: isabel.rebollido@uam.es

2 Observatorio Astrónomico de Calar Alto, CAHA, 04550 Gérgal, Almería, Spain

${ }^{3}$ Centro de Astrobiología (CAB, CSIC-INTA), ESAC Campus Camino Bajo del Castillo, s/n, Villanueva de la Cañada, 28692 Madrid, Spain

${ }^{4}$ INAF, Osservatorio Astronomico di Palermo, Piazza del Parlamento 1, 90134 Palermo, Italy

5 STAR Institute, Université de Liège, F.R.S.-FNRS, 19c Allée du Six Août, 4000 Liège, Belgium

6 Instituto de Física y Astronomía, Facultad de Ciencias, Universidad de Valparaíso, 5030 Casilla, Valparaíso, Chile

7 Núcleo Milenio de Formación Planetaria - NPF, Universidad de Valparaíso, Av. Gran Bretaña 1111, Valparaíso, Chile

8 European Space Astronomy Centre (ESA), PO Box 78, 28691 Villanueva de la Cañada, Madrid, Spain

9 Université de Toulouse, UPS-OMP, IRAP, Toulouse 31400, France

10 Space Telescope Science Institute, 3700 San Martin Drive, Baltimore, MD 21212, USA

11 Steward Observatory, Department of Astronomy, University of Arizona, Tucson, AZ 85721, USA

12 Max-Planck-Institut für Astronomie (MPIA), Königstuhl 17, 69117 Heidelberg, Germany

13 Department of Space, Earth and Environment, Chalmers University of Technology, Onsala Space Observatory, 43992 Onsala, Sweden

14 Konkoly Observatory, Research Centre for Astronomy and Earth Sciences, Konkoly-Thege Miklós út 15-17, 1121 Budapest, Hungary

15 ELTE Eötvös Loránd University, Institute of Physics, Pázmány Péter sétány 1/A, 1117 Budapest, Hungary

16 Aurora Technology B.V. for ESA, ESA-ESAC, Villanueva de la Cañada, 28691 Madrid, Spain

17 Observatorio Astronómico Nacional (OAN-IGN)-Observatorio de Madrid, Alfonso XII, 3, 28014 Madrid, Spain

Received 11 June 2019 / Accepted 23 March 2020

\begin{abstract}
Context. While exoplanets are now routinely detected, the detection of small bodies in extrasolar systems remains challenging. Since the discovery of sporadic events, which are interpreted to be exocomets (falling evaporating bodies) around $\beta$ Pic in the early 1980 s, only $\sim 20$ stars have been reported to host exocomet-like events.

Aims. We aim to expand the sample of known exocomet-host stars, as well as to monitor the hot-gas environment around stars with previously known exocometary activity.

Methods. We have obtained high-resolution optical spectra of a heterogeneous sample of 117 main-sequence stars in the spectral type range from B8 to G8. The data were collected in 14 observing campaigns over the course of two years from both hemispheres. We analysed the Ca II K\&H and Na I D lines in order to search for non-photospheric absorptions that originated in the circumstellar environment and for variable events that could be caused by the outgassing of exocomet-like bodies.

Results. We detected non-photospheric absorptions towards $50 \%$ of the sample, thus attributing a circumstellar origin to half of the detections (i.e. $26 \%$ of the sample). Hot circumstellar gas was detected in the metallic lines inspected via narrow stable absorptions and/or variable blue- and red-shifted absorption events. Such variable events were found in 18 stars in the Ca II and/or Na I lines; six of them are reported in the context of this work for the first time. In some cases, the variations we report in the Ca II K line are similar to those observed in $\beta$ Pic. While we do not find a significant trend in the age or location of the stars, we do find that the probability of finding CS gas in stars with larger $v \sin i$ is higher. We also find a weak trend with the presence of near-infrared excess and with anomalous ( $\lambda$ Boo-like) abundances, but this would require confirmation by expanding the sample.
\end{abstract}

Key words. stars: general - comets: general - Kuiper belt: general - ISM: clouds

\section{Introduction}

Main sequence (MS) stars are known to host a complex circumstellar (CS) environment populated by a plethora of planets, debris discs, and minor bodies, which are inherited from the physics that regulates the formation of stars. Later, the mutual

^ Table C.1 is only available at the CDS via anonymous ftp to cdsarc.u-strasbg.fr (130.79.128.5) or via http://cdsarc. u-strasbg.fr/viz-bin/cat/J/A+A/639/A11 dynamical interaction among those bodies and their host stars determines the evolution of the planetary systems.

Since the discovery of a giant planet orbiting the solar-type star 51 Peg (Mayor \& Queloz 1995), thousands of planets have been detected, which make up planetary systems with diversified architectures (see e.g. The Extrasolar Planets Encyclopae$\mathrm{dia}^{1}$ ). Minor bodies, such as asteroids and comets, are expected in these planetary systems. Their study is relevant as they

\footnotetext{
1 exoplanet.eu
} 
provide clues for understanding the formation and dynamical evolution of planetary systems (e.g. Armitage 2010). However, evidence of their presence are practically limited to indirect observations, such as the detection of circumstellar dust and gas in debris discs and, to a less considerable extent, to more direct evidence, such as observations of some metallic line absorptions.

Hundreds of debris discs are currently known to surround MS stars across practically all spectral types and ages from around $10 \mathrm{Myr}$ to several gigayears. Debris discs are detected as thermal emission at mid-IR, far-IR, (sub-)millimetre wavelengths, as well as scattered light in the optical and near-IR from small dust particles, which mainly originate in collisions among kilometre-sized planetesimals and other destructive processes (e.g Matthews et al. 2014, and references therein). It has been suggested that at least a fraction of debris discs posses both warm $(T \sim 200 \mathrm{~K})$ and cold $(T \lesssim 100 \mathrm{~K})$ dust belts (e.g. Ballering et al. 2013; Chen et al. 2014; Kennedy \& Wyatt 2014; Pawellek et al. 2014; Morales et al. 2016), which is reminiscent of the Solar asteroid and Kuiper belts, respectively. The two-belt structure could be created by a chain of planets, while comets scattered by those planets could constitute a relevant source feeding the warm exozodiacal belt (Schüppler et al. 2016; Geiler \& Krivov 2017; Marino et al. 2018). In our own Solar System, cometary material from Jupiter-family comets is responsible for replenishing the zodiacal cloud (Nesvorný et al. 2010).

Interferometric studies (Absil et al. 2006, 2013; Ertel et al. 2014; Nuñez et al. 2017) have revealed near-IR excesses that are also attributed, in most cases, to the combination of thermal emission and scattered light from small sub-micronsized, hot $(T \sim 1500-2000 \mathrm{~K})$ dust particles located within 0.01-1 AU from the stars (depending on the luminosity, see Kirchschlager et al. 2017), which are very close to the dust sublimation zone. Again, cometary bodies that are scattered inwards from an outer reservoir are a likely scenario as to the origin and persistence of the hot dust (Beust \& Morbidelli 2000; Thébault \& Beust 2001; Bonsor et al. 2014; Marboeuf et al. 2016; Faramaz et al. 2017; Marino et al. 2018; Sezestre et al. 2019).

Significant amounts of cold gas at several tens of astronomical units from the central stars (most young A-type stars) have been detected around $\sim 20$ bright debris discs (e.g. Moór et al. 2015a, 2017; Riviere-Marichalar et al. 2012, 2014; Roberge et al. 2013; Greaves et al. 2016; Lieman-Sifry et al. 2016; Marino et al. 2016). The cold gas most likely has a secondary origin (e.g. Marino et al. 2016; Matrà et al. 2017; Kral et al. 2019), but in some cases a remnant of the primordial protoplanetary disc has been suggested (Kóspál et al. 2013; Moór et al. 2015a; Kral et al. 2017). A variety of physical processes, including outgassing of cometary objects, have been invoked to explain the secondary cold gas; all of them are related to the presence of planetesimals (e.g. Matthews et al. 2014, and references therein). In parallel, UV and optical high resolution spectroscopy reveals non-photospheric stable absorption features of elements, such as $\mathrm{C}, \mathrm{O}, \mathrm{Ca}, \mathrm{Na}$, or $\mathrm{Fe}$, at or close to the radial velocity of the star, as well as weak sporadic red- or blue-shifted absorption events with respect to the stellar radial velocity (Vidal-Madjar et al. 1994, 2017; Lagrange et al. 1998; Brandeker et al. 2004; Roberge et al. 2006; Iglesias et al. 2018; Rebollido et al. 2018). This gas would be hot with temperatures from $\sim 1000-2000 \mathrm{~K}$ (Hobbs et al. 1988; Beust et al. 1998; Vidal-Madjar et al. 2017). The transient absorptions have been interpreted as hot gas that is released by the evaporation of exocomets grazing or falling onto the star, which is referred to as the "falling evaporating bodies" scenario or FEBs (e.g. Ferlet et al. 1987; Beust et al. 1990), while grain evaporation in the circumstellar disc has also been proposed to explain the stable absorption components (e.g. Fernández et al. 2006). A trend between the detection of this hot gas and the edge-on orientation of cold-gas-bearing debris discs was found by Rebollido et al. (2018), who attributes it to a geometrical effect. It is remarkable that this hot gas would be located at distances $\lesssim 0.5 \mathrm{AU}$ from the star, that is, at similar distances as the hot exozodiacal dust.

The first direct evidence for the presence of exocomets (FEBs) was found around $\beta$ Pic, which remains unique and the best theoretically and observationally studied case (e.g. Ferlet et al. 1987; Beust et al. 1991; Kiefer et al. 2014a; Vidal-Madjar et al. 2017, and references therein). Several hundreds of cometary transits in $\beta$ Pic have been detected, revealing two families of exocomets with distinct dynamical and compositional properties; one likely formed by old comets that were strongly depleted in volatiles and a second one that could be related to the recent fragmentation of one or a few parent bodies (Kiefer et al. 2014a). It is worth noting that very recently, $\beta$ Pic transiting exocomets were likely detected by means of TESS broadband photometry (Zieba et al. 2019). Those exocomets would have been driven into the vicinity of the star by a larger body, that is, a planet (Beust et al. 1991; Beust $\&$ Morbidelli 2000). Thus, FEBs constitute an indicator of the plausible presence of planets; we recall that the Jupiter-like planet $\beta$ Pic b was later revealed by imaging (Lagrange et al. 2010).

In addition to $\beta \mathrm{Pic}$, absorption events, mainly in the $\mathrm{Ca}$ II $\mathrm{K}$ line that is accompanied in most cases by a stable component, have been detected towards more than 20 stars (e.g. Redfield 2007; Kiefer et al. 2014b; Welsh \& Montgomery $2015,2018)$. Those stars are usually young ( $<100 \mathrm{Myr})$ A-type stars, but transient features have also been found around older stars. Also, Welsh \& Montgomery (2019) have recently reported the first detection of an exocomet-like event with a $2.9 \sigma$ signal around the F2 V type star $\eta \mathrm{Crv}$. In addition, Kepler photometric light curves have been explained to be due to transiting exocomets in a few F- and later spectral-type stars (Rappaport et al. 2018; Ansdell et al. 2019) and maybe Boyajian's star (Boyajian et al. 2016). Exocomet-host stars have large projected rotational velocities, most with $v \sin i \gtrsim$ $100 \mathrm{~km} \mathrm{~s}^{-1}$, in principle suggesting that the systems are viewed close to edge-on, which is the favoured orientation to detect non-photospheric absorptions from comet-like bodies passing in front of the star and is consistent with the trend suggested by Rebollido et al. (2018). In some cases, the stars hosting exocomets are associated with a debris disc. We note, however, that scattering of exocomets by eccentric planets can take place in planetary systems with low luminous, non-detectable debris discs with flux levels comparable to the Kuiper belt (Faramaz et al. 2017).

This work presents the observational results of a high spectral resolution survey of a large sample of stars. The primary aim is to detect and monitor non-photospheric absorption features due to exocomets that pass in front of the stars. The secondary goal is to assess any potential trend between the presence of exocomets and the properties of the host stars. The paper is structured as follows. Section 2 describes the sample of stars, the criteria to select them, and some basic properties. Section 3 presents our spectroscopic observations and data analysis. Section 4 presents our spectroscopic results concerning non-photospheric stable and variable features, their plausible interstellar (ISM) or circumstellar (CS) origin, and comments in respect to some individual stars. Section 5 discusses the detection of the non-photospheric absorptions regarding some stellar properties as well as with respect to the selection criteria. Finally, Sect. 6 presents the conclusions of this work. 


\section{Sample}

As mentioned above, the primary goals of our study are the detection and monitoring of variable, non-photospheric absorption features that, as in the case of $\beta$ Pic, could be attributed to the evaporation of solid bodies in the immediate surroundings of main-sequence stars. The observed stellar sample is formed by a heterogeneous and biased set of 117 MS stars in the spectral type range from B8 to G8, which is aimed at optimising those goals. The targets were selected according to the following criteria: i) stars with previously reported $\mathrm{Ca}$ II H\&K and/or Na I D events attributed to exocomets; ii) debris disc stars, seen edge-on when known; iii) debris disc stars where the presence of cold gas has been detected at far-IR and (sub-)millimetre wavelengths; iv) stars with near-IR excesses that could be due to hot dust; v) stars belonging to young associations, namely Upper Scorpius (UpSco), Tucana-Horologium (Tuc-Hor), and the $\beta$ Pictoris moving group (BPMG); vi) shell stars with circumstellar Ti II absorptions; and vii) $\lambda$ Bootis stars. The Ti II stars were selected because those lines denote the presence of discs seen at nearly right angles to the rotational axes (Abt \& Moyd 1973; Abt et al. 1997), that is, hot discs seen near edge-on. We note that, in general, shell stars are a heterogenous group of late B- to early F-type stars with the distinct characteristic of enhanced lines of Fe II and Ti II, which denote the presence of a gaseous circumstellar shell (Gray \& Corbally 2009, and references therein). In addition, $\lambda$ Bootis stars are A and early $\mathrm{F}$ spectral type stars, which are strongly depleted in heavier elements (such as Fe, Al, $\mathrm{Mg}$, and $\mathrm{Ca}$ ), and relatively normal abundances of volatile elements, such as C, N, O, and S (Paunzen 2004). It is important to note that some of them show clear evidence of accreting CS gas in their UV and optical spectra (Grady et al. 1996a; Holweger et al. 1999). The $\lambda$ Bootis abundance pattern is most likely due to the selective accretion of the volatile elements onto the star, that is, material that could be provided by exocomets, although other sources could also be an alternative (Jura 2015; Draper et al. 2016). We note that some stars in the sample share several of the selection criteria.

A detailed list of the stars in the sample and their properties and selection criteria are listed in Table B.1. Figure 1 shows the spatial distribution of the sample in galactic coordinates. Given the characteristics of the selection criteria, there is no preferencial location with the exception of stars in the UpSco and Tuc-Hor young associations, although most stars are in the Southern Hemisphere.

\section{Observations and data analysis}

High-resolution observations were taken in a series of campaigns from August 2015 to September 2017 from both the Northern and Southern Hemispheres, using different fibre-fed spectrographs and telescopes. The spectrographs HERMES (Raskin et al. 2011), which is attached at the $1.5 \mathrm{~m}$ Mercator Telescope, and FIES (Frandsen \& Lindberg 2000) at the $2.5 \mathrm{~m}$ Nordic Optical Telescope (NOT) were used in El Roque de los Muchachos Observatory (Canary Islands, Spain). In the La Silla Observatory (Chile), the FEROS spectrograph (Kaufer et al. 1999) at the MPG/ESO 2.2 telescope was used. Complementary observations were obtained at the La Luz Observatory (México) with the TIGRE telescope and the HEROS spectrograph (Schmitt et al. 2014). Table 1 summarises the resolutions

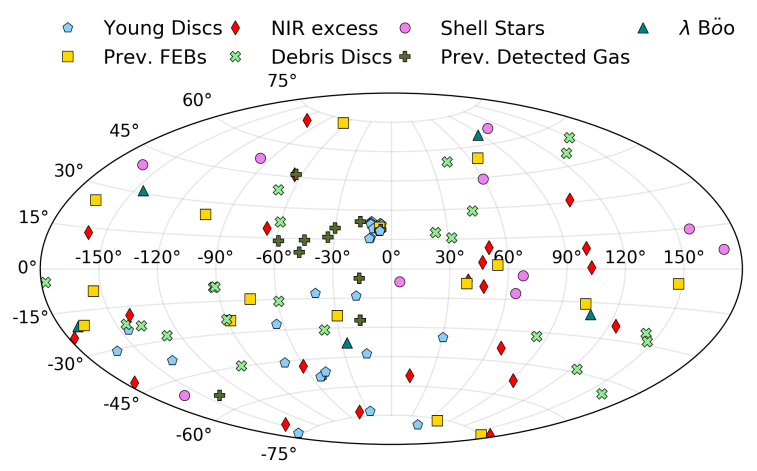

Fig. 1. All sky plot of the sample in galactic coordinates. Although most stars are in the Southern Hemisphere, there is no preferential spatial location, with the exception of stars in the UpSco and Tuc-Hor young associations.

and wavelength ranges of the various instruments as well as the observing campaigns. A total of 1575 spectra for the 117 stars were obtained; a detailed observing log with the specific dates (UT) of the spectra for each star and the corresponding spectrograph can be found in Table C.1, available in electronic form in CDS. Due to the nature of the irregular, sporadic exocometarylike events, we aimed to obtain time-series spectra, with at least one spectrum per night per object when possible. Integration times never exceeded $30 \mathrm{~min}$ and were mainly selected depending on the telescope and brightness of the star with the general goal of obtaining a signal-to-noise ratio $(S / N) \gtrsim 100$ in the $\mathrm{Ca}$ II H\&K lines. That goal was not always achieved due to either poor weather conditions or the faintness of the star. The $\mathrm{S} / \mathrm{N}$ of the median spectra for each star measured in the continuum close to $\mathrm{Ca}$ II $\mathrm{K}$ line is given in Table C.1.

Data reduction was performed by the available pipelines of the different spectrographs. The reduction includes the usual steps for echelle spectra as bias subtraction, flat-field correction, cosmic ray removal, and order extraction; wavelength calibration was carried out by means of Th-Ar lamp spectra. In addition, barycentric corrections for the HERMES and FIES spectra were carried out since the corresponding pipelines do not include such a correction. All spectra were normalised and the continuum was set to 1.0 in the regions between spectral lines.

\subsection{Telluric subtraction}

The observed wavelength range includes regions of the visible spectra that are heavily affected by telluric lines and, in particular, the region around the 5890/5896 $\AA \mathrm{Na}$ I D lines, which are highly relevant for both interstellar and circumstellar absorptions. The removal of telluric lines was performed by means of MOLECFIT $^{2}$ (Smette et al. 2015; Kausch et al. 2015), a tool that generates an atmosphere model accounting for the most common absorbing molecules in the optical range $\left(\mathrm{H}_{2} \mathrm{O}, \mathrm{O}_{2}, \mathrm{O}_{3}\right)$. Residuals after subtraction of the atmosphere model in the telluric line region are comparable to the noise level, and they are therefore negligible. An example of telluric subtraction is shown in Fig. 2.

\subsection{Stellar parameters}

Stellar parameters $T_{\text {eff }}, \log g$, and $v \sin i$ for the early type stars (up to F2) in the sample were computed using the procedure

\footnotetext{
2 https://www.eso.org/sci/software/pipelines/skytools/
} molecfit 
Table 1. Instruments and observing campaings.

\begin{tabular}{lllll}
\hline \hline & HERMES & FIES & FEROS & HEROS ${ }^{(!)}$ \\
\hline Resolution & $\sim 85000$ & $\sim 67000$ & $\sim 48000$ & $\sim 20000$ \\
Range (nm) & $377-900$ & $364-736$ & $352-920$ & $374-884$ \\
\hline Observations & 03-06/Sep/2015 & $26-27 / \mathrm{Jan} / 2016$ & $21-24 / \mathrm{Oct} / 2015$ & Aug 2015 \\
& 20-23/Dec/2015 & $16-19 / \mathrm{Jul} / 2016$ & $25-28 / \mathrm{Mar} / 2016$ & Sept 2015 \\
& 27-30/Jan/2016 & 03/Mar/2016 ${ }^{(*)}$ & $31 / \mathrm{Mar}-08 /$ Apr/2017 & Oct 2015 \\
& 03-06/Mar/2016 & 21/Mar/2016 ${ }^{(*)}$ & $23 / \mathrm{Sep}-01 /$ Oct/2017 & Nov 2015 \\
& 08-11/May/2016 & & & Dec 2015 \\
& 11-14/Jul/2016 & & & Jan 2016 \\
& 06-03/Apr/2017 & & & \\
& 28/Mar-03/Apr/2017 & & & \\
\hline
\end{tabular}

Notes. ${ }^{(*)}$ On March 3 and 21 during service mode, only one spectrum was obtained each night. ${ }^{(!)}$HEROS spectra were taken in robotic mode over the course of several months as complementary observations.

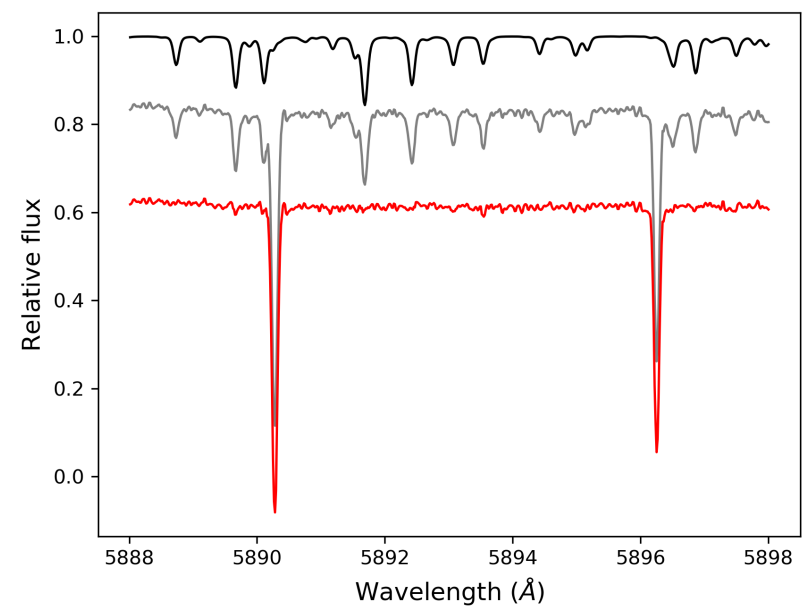

Fig. 2. Example of telluric subtraction in HD 21620 in the Na I D spectral region. The black line shows the MOLECFIT atmospheric model, the grey line shows the observed spectrum, and the red line shows the final telluric-free HD 21620 spectrum.

described by Rebollido et al. (2018). Solar abundances were initially used to iterate the solutions for all objects, the solutions being consistent with the metallicity for most of the stars. For the 15 objects with $[M / H] \leq-0.5$, according to our estimates, eight of them, namely HD 31295, HD 74873, HD 110411, HD 125162 ( $\lambda$ Boo), HD 183324, HD 198160 (HR 7959), HD 198161 (HR 7960), and HD 221756, were classified as $\lambda$ Boo stars by Murphy et al. (2015), which is consistent with their expected underabundance in heavier elements.

For cooler stars (later than F2), a different approach was used to calculate the parameters. In those cases, we followed the procedure described in detail by Maldonado \& Villaver (2017) and Maldonado et al. (2018), which is based on the iron ionisation, excitation equilibrium, and match of the curve of growth conditions.

Radial velocities $\left(v_{\mathrm{rad}}\right)$ were estimated by measuring the shift between the synthetic spectrum, which is computed using a database of rest wavelengths, and the observed spectrum that is corrected for barycentric velocity. Individual shifts were measured for the Balmer lines from $\mathrm{H} \gamma$ to $\mathrm{H} 9$. Additionally, $\mathrm{H} \epsilon$, which is blended with $\mathrm{Ca}$ II $\mathrm{H}$, was excluded. Lines that are bluer than $\mathrm{H} 10$ were discarded since the lower part of the absorptions were usually noisy. Most of the stars have large values for

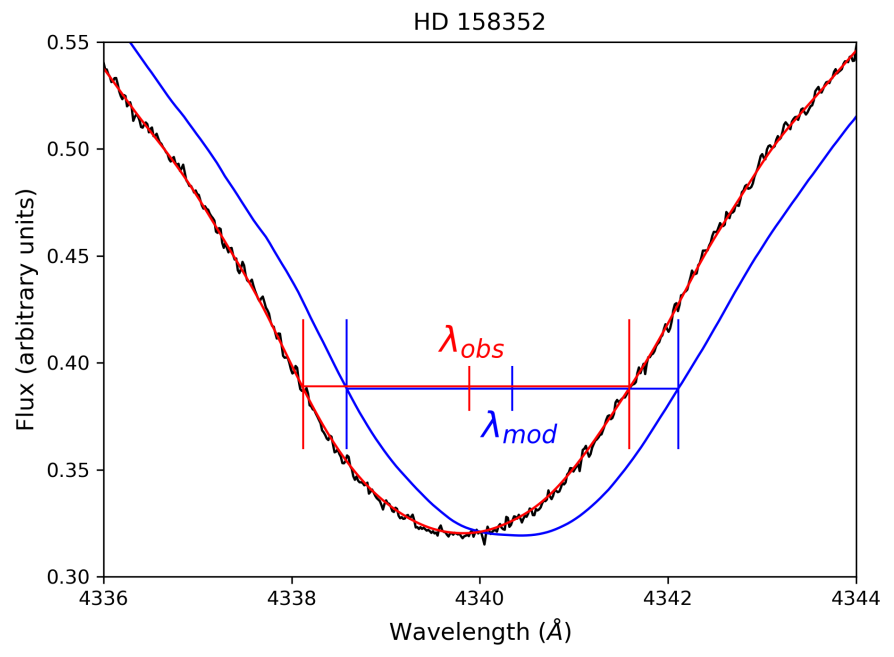

Fig. 3. Radial velocities are measured as the difference between the synthetic model at rest (blue line) and the smoothed spectra at the radial velocity of the star (red line). The method is illustrated in the plot by means of the observed $\mathrm{H} \gamma$ line in the star HD 158352 (black line). A detailed description is given in the text.

the projected rotational velocity, $v \sin i$; therefore, the cores of the lines and, in particular, the Balmer ones are fairly rounded. Thus, a direct evaluation of the wavelength at which the minimum intensity occurs introduces large uncertainties. The procedure we followed is to slightly smooth the spectra and then to use, as the reference wavelength, that of the bisector corresponding to $10 \%$ of the line intensity measured from the bottom of the absorption. The same was done on the synthetic profile and the difference was converted into $v_{\text {rad }}$. The results do not change significantly if, instead of using the synthetic spectrum, the rest laboratory wavelength of the particular Balmer line was used as a reference. The $v_{\text {rad }}$ uncertainties come from the standard deviation of the set of displacements. Figure 3 shows the method explicitly.

\subsection{Non-photospheric absorptions}

The Ca II H\&K and Na I D lines were visually inspected to search for the presence of non-photospheric, stable or variable, absorption features at the core of the photospheric lines, which would suggest the presence of circumstellar gas. A median 
Table 2. Stars with variable non-photospheric absorption features. References are within brackets.

\begin{tabular}{|c|c|c|}
\hline Name & Prev. detected & Det. in this work \\
\hline HD $256(\text { HR } 10)^{(*)}$ & Yes $(1,12,15)$ & Yes \\
\hline HD 9672 (49 Cet) & Yes (2) & No \\
\hline HD 21620 & Yes (3) & Yes \\
\hline HD 32297 & Yes (4) & No \\
\hline HD 36546 & No & Yes \\
\hline HD 37306 & No & Yes \\
\hline HD 39182 (HR 2025) & No & Yes \\
\hline HD 42111 & Yes $(5,12)$ & Yes \\
\hline HD 50241 & Yes $(5,11)$ & No \\
\hline HD $56537(\lambda$ Gem $)$ & Yes (6) & No \\
\hline HD 64145 ( $\phi \mathrm{Gem})$ & Yes (6) & No \\
\hline HD 80007 (HR 3685) & Yes $(11,15)$ & Yes \\
\hline HD 85905 & Yes $(7,15)$ & Yes \\
\hline HD $98058(\phi \text { Leo })^{(*)}$ & No & Yes \\
\hline HD 108767 ( $\delta$ Crv $)$ & Yes (6) & No \\
\hline HD 109573 (HR 4796) & Yes $(6,16)$ & Yes \\
\hline HD 110411 & Yes (3) & Yes \\
\hline HD 138629 (HR 5774) & Yes (8) & No \\
\hline HD $132200^{(*)}$ & No & Yes \\
\hline HD 145964 & Yes (3) & Yes \\
\hline HD 148283 (HR 6123) & Yes $(5,13)$ & No \\
\hline 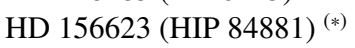 & No & Yes \\
\hline HD 172555 & Yes (9) & Yes \\
\hline HD 182919 (5 Vul) & Yes (2) & Yes \\
\hline HD 183324 & Yes $(10,16)$ & Yes \\
\hline HD 217782 & Yes $(2,5,14)$ & Yes \\
\hline
\end{tabular}

Notes. ${ }^{(*)}$ Results have been presented by Eiroa et al. (2016), Rebollido et al. (2018), and Montesinos et al. (2019).

References. (1) Lagrange-Henri et al. (1990a); (2) Montgomery \& Welsh (2012); (3) Welsh \& Montgomery (2013); (4) Redfield (2007); (5) Roberge \& Weinberger (2008); (6) Welsh \& Montgomery (2015); (7) Welsh et al. (1998); (8) Lagrange-Henri et al. (1990b); (9) Kiefer et al. (2014b); (10) Montgomery \& Welsh (2017); (11) Hempel \& Schmitt (2003); (12)Lecavelier Des Etangs et al. (1997); (13) Grady et al. (1996b); (14) Cheng \& Neff (2003); (15) Redfield et al. (2007); (16) Iglesias et al. (2018)

spectra was constructed for each star to improve the $\mathrm{S} / \mathrm{N}$ in order to analyse the potential stable absorption. It is important to specify that when data from different telescopes were available, all spectra were converted to the FEROS resolution in order to construct the median. This analysis was carried out by estimating the photospheric contribution with splines fitted to the bottom of the lines, then by dividing the observed profiles by the estimated photospheric lines, and finally by fitting Gaussians to the residuals.

A total of 60 stars show stable, non-photospheric Ca II and/or Na I absorptions (Table B.3); at the same time, irregular, variable features are seen in individual spectra of 18 stars (Table 2). For these latter stars, when no variations were detected in a range of hours or days, a median was constructed including all the consecutive non-variating spectra. The stable absorptions, when arising in the CS environment (see Sect. 4.1.1), appear at the radial velocity $\left(v_{\mathrm{rad}}\right)$ of the stars or close to it, while the variable ones appear red- and/or blue-shifted. In the following, we present a separate analysis for stars only showing stable nonphotospheric absorptions and stars with variable components regardless of whether they also have stable absorptions.

\section{Results}

\subsection{Narrow, stable absorption features}

As mentioned above, a total of 60 out of the 117 stars in the sample show narrow, stable absorption features superimposed on the photosphere, either in the $\mathrm{Ca}$ II and/or the $\mathrm{Na}$ I lines. Figure A. 1 shows the observed line profiles for both the Ca II $\mathrm{H} \& \mathrm{~K}$ and $\mathrm{Na}$ I D lines of the 60 stars, along with their residuals, once the photosphere has been divided. The characteristics of the absorptions are listed in Table B.3. EWs were calculated with respect to the adjacent continuum once it was divided by the photospheric contribution. The EW ratio for the $\mathrm{Ca}$ II and $\mathrm{Na}$ I doublets expected from the atomic properties of the transition (range $0.5-1.0$ in both cases encompassing the optically thin and optically thick regimes) is not always maintained due to the EW uncertainties and the very complex geometry and composition of the CS gas. Column densities were estimated following Somerville (1988) in order to deal with the saturated lines. Uncertainties for the features' radial velocities are estimated to be two pixels of the median spectra, corresponding to $\sim 2.5 \mathrm{~km} \mathrm{~s}^{-1}$ in Ca II lines and $\sim 1.5 \mathrm{~km} \mathrm{~s}^{-1}$ in Na I lines, and this value is consistent with the FWHM of the lines of the calibration lamps. In the case of EWs, we estimate an uncertainty of $10 \%$ in our measurements. Uncertainties for column densities were calculated as propagation of the EW uncertainties according to the formulas for the non-saturation and saturation cases (Eqs. (1) and (7) in Somerville 1988, respectively). In some stars, most were previously classified as shell stars, the $\mathrm{Ca}$ II $\mathrm{K}$ line has a sharp, very pronounced triangular-like profile consisting of a very narrow core and broader wings, which cannot be fitted by either a Gaussian or a Voigt profile. In those cases, we measured the EWs of the excess absorption with respect to the photospheric line and the velocity dispersion as the FWHM of the mentioned excess absorption, that is, the velocity dispersion at one half of the absorption depth.

\subsubsection{Origin of the non-photospheric stable absorptions}

Stable non-photospheric absorption features might originate in the close-in CS environment of the star or in the warm and cold clouds of the local interstellar medium (ISM, e.g. Redfield \& Linsky 2008). In order to try to decipher the origin, we compared the radial velocity of those features with the radial velocities $\left(v_{\mathrm{rad}}\right)$ of the corresponding stars (Tables B.2 and B.3) and with the velocity vectors of the local ISM $\left(v_{\text {ISM }}\right)$ towards the line of sight of each star, as given for the ISM clouds in Redfield \& Linsky's Colorado model ${ }^{3}$. Velocities and names of the clouds are also given in Table B.3. Nonetheless, a sound ascription to CS or ISM origins is ambiguous in some cases; for instance, when the stellar $v_{\text {rad }}$ and the ISM $v_{\text {ISM }}$ along its line of sight are very close, or when there is no identified ISM Colorado cloud along the line of sight towards some stars with narrow $\mathrm{Ca}$ II and $\mathrm{Na}$ I absorptions. Furthermore, the properties of the nonphotospheric absorptions do not clearly discriminate the plausible origin (Redfield et al. 2007) since the observed EWs in the ISM of the Ca II H\&K and of the Na I D lines as well as their ratios vary by several orders of magnitude (e.g Welty et al. 1996; Redfield \& Linsky 2008; Welsh et al. 2010). We note that Na I is found in cold ISM gas $(\sim 50 \mathrm{~K})$, which is typically at distances larger than $\sim 80 \mathrm{pc}$ (Welsh et al. 2010). Although, it is also occasionally found at shorter distances (e.g. Bertin et al. 1993; Welty et al. 1994), whereas Ca II appears in a much warmer medium

\footnotetext{
3 http://sredfield.web.wesleyan.edu/
} 
$(\sim 5000 \mathrm{~K})$ and is usually detected at much shorter distances (e.g. Redfield \& Linsky 2008).

In this context, and by taking the mentioned caveats into account, we identify those stars with CS gas that satisfy any of the following criteria: (i) stars with variable absorptions (see Sect. 4.2); (ii) stars where the non-photospheric absorption shares its velocity with the radial velocity of the star but not with any ISM Colorado cloud; and (iii) stars with Ti II absorption lines and shell stars where the Ca II K line has a sharp, triangular-like profile. We point out that some stars have more than one non-photospheric feature that can be independently identified with the velocity of the star or of the ISM. Also, for some stars, the absorptions neither coincide with $v_{\text {rad }}$ nor with $v_{\text {ISM. }}$. When possible, an inspection for nearby stars was made in a field of $5^{\circ}$ in radius and distances $\pm 20 \mathrm{pc}$ around each star. A column in Table B.3 shows our guess as to the origin of the non-photospheric absorptions.

\subsubsection{Comments on individual stars}

In this section we discuss the plausible origin of the stable nonphotospheric absorptions for some individual stars or group of stars. Stars with variable absorption features detected in this work are discussed in Sect. 4.2. HD 9672 (49 Cet), HD 32297, HD 110058, HD 131488, HD 131835, HD 138813, HD 146897 , HD 156623, HD 172555, and HD 181296 ( $\eta$ Tel) are analysed in Rebollido et al. (2018); therefore, we do not repeat the discussion here. We note, however, that $\mathrm{Na}$ I D features in the stars HD 9672, HD 156623, and HD 172555 were erroneously omitted by Rebollido et al. (2018). A corrigendum can be found in Rebollido et al. (2019). We point out that this mistake does not affect the main conclusions of Rebollido et al. (2018). The Na I D features are nonetheless included in Table B.3 and shown in Fig. A.1. In HD 9672, the $\mathrm{Na}$ I D absorption appears at the velocity of the $\mathrm{Ca}$ II feature, the radial velocity of the star, and the velocity vector of the local interstellar cloud (LIC) cloud. In the case of HD 156623, the Na I D feature appears in emission at the stellar $v_{\text {rad. }}$ For HD 172555, two Na I D2 components appear; one is close to the $\mathrm{G}$ cloud, which is in agreement with Kiefer et al. (2014b), and a second one is very broad and variable (see Sect. 4.2).

- HD 2884, HD 2885, and HD 3003 are members of the Tuc-Hor association. All three stars have similar proper motions, radial velocities, and parallaxes. The projected angular separation between HD 2884 and HD 2885 is $\sim 27^{\prime \prime}$, while the one between HD 2884 and HD 3003 is $\sim 9^{\prime}$. Thus, they likely form a physical multiple system (see also Eggleton \& Tokovinin 2008; Tokovinin 2008; Howe \& Clarke 2009). HD 2885 itself is a binary candidate (Lagrange et al. 2009). Our spectra show a weak narrow $\mathrm{Ca}$ II $\mathrm{K}$ absorption in HD 2884, a weak Na I D absorptions towards HD 2885, and weak Ca II K and $\mathrm{Na}$ I D absorptions towards HD 3003 (see also Iglesias et al. 2018). All these absorptions are close to the velocity vector of the Vel cloud and do not coincide with the $v_{\text {rad }}$ of the stars (Table B.3 and Fig. A.1). HD 224392 is another Tuc-Hor star located at a similar distance from the Sun as the multiple system and at an angular separation of $\sim 4 \mathrm{deg}$. Its spectrum shows non-photospheric absorptions of both $\mathrm{Ca}$ II and $\mathrm{Na}$ I. The velocity of the Ca II feature is close to the Vel cloud and to the $\mathrm{Ca}$ II absorptions in HD 2884 and HD 3003; the velocity of the Na I is close to the Cet Colorado cloud that passes at $<20^{\circ}$ the line of sight of HD 224392. All these facts suggest an ISM origin for the Ca II and $\mathrm{Na}$ I features; however, this ascription is controversial. First, we note that neither $\mathrm{Ca}$ II nor $\mathrm{Na}$ I non-photospheric features appear simultaneously in HD 2884 or HD 2885. At the same time, Na I was detected towards HD 3003 but not towards HD 2884. Thus, since all three stars are located very close, in particular HD 2884 and HD 2885, a CS origin for the observed features cannot be excluded. Given all these facts, we find it ambiguous to ascribe the non-photospheric features in all four stars to either a CS or ISM origin and further observations are needed to elucidate their origin.

- HD 5267 has a strong non-photospheric feature at $\sim-5.0 \mathrm{~km} \mathrm{~s}^{-1}$ in both $\mathrm{Ca}$ II and $\mathrm{Na}$ I lines. The velocity of this feature does not coincide with $v_{\text {rad }}\left(9.5 \mathrm{~km} \mathrm{~s}^{-1}\right)$ or with the $v_{\text {ISM }}$ of any Colorado clouds. At the same time, there is a weak Ca II $\mathrm{K}$ feature at $\sim 10.0 \mathrm{~km} \mathrm{~s}^{-1}$, which was also tentatively detected in the $\mathrm{Na}$ I D2 line, close to stellar radial velocity and the velocity vector, $11.44 \mathrm{~km} \mathrm{~s}^{-1}$, of the LIC cloud (Table B.3). Unfortunately there are no spectra of field stars in the ESO archive that can be used to discriminate the origin of the component at $-5 \mathrm{~km} \mathrm{~s}^{-1}$. We note, however, that this feature is $\sim 15 \mathrm{~km} \mathrm{~s}^{-1}$ blue-shifted relative to the radial velocity of the star and that HD 5267 has a similar effective temperature as HD 181296, $T_{\text {eff }} \sim 10500 \mathrm{~K}$. HD 181296 has a stable component $\sim 20 \mathrm{~km} \mathrm{~s}^{-1}$ blue-shifted relative to the stellar velocity, which is most likely of CS origin as suggested in Rebollido et al. (2018) (see Chen \& Jura 2003, for other examples). Thus, while the origin of the $\sim 10 \mathrm{~km} \mathrm{~s}^{-1}$ component is ambiguous, the origin of the $-5 \mathrm{~km} \mathrm{~s}^{-1}$ is unknown.

- HD 16978 is another Tuc-Hor star reported by Welsh \& Montgomery (2018) to have a variable non-photospheric Ca II $\mathrm{K}$ profile within three observations. Our spectra show such an absorption with a similar EW, but it does not show the mentioned variability. The radial velocity of the feature coincides with the stellar $v_{\text {rad }}$, and it differs $\sim 4 \mathrm{~km} \mathrm{~s}^{-1}$ from the $v_{\text {ISM }}$ of the Vel cloud. Given the variability of the feature profile noted by Welsh \& Montgomery (2018), which might be indicative of a transient event, the more plausible origin is CS, although we cannot definitively exclude an ISM origin.

- The stars HD 71043, HD 71722, and HD 105850 have Ca II and $\mathrm{Na}$ I features that are close to the velocity vectors of Colorado clouds, and they are far away form the stellar $v_{\text {rad }}$, supporting an ISM origin, which is in agreement with Iglesias et al. (2018). This is also the case for the star HD 188228 where only a weak $\mathrm{Na}$ I absorption is seen close to the velocity of the $\mathrm{G}$ and Vel clouds.

- HD 118232 (24 CVn), HD 125162 ( $\lambda$ Bootis), and HD 221756 have non-photospheric absorption features, which do not coincide with either the stellar radial velocities or any known ISM Colorado clouds. Due to their high declinations $\left(>+40^{\circ}\right)$, we did not find any spectra of field stars in the ESO archive that could help to elucidate the origin of the absorptions. Nonetheless, we find that an ISM origin is the most plausible one given the remarkable shift in velocity between the features and the photospheric lines.

- HD 145689 (HIP 79797) is a member of the Argus association and shows a weak Ca II absorption at a velocity of $11.9 \mathrm{~km} \mathrm{~s}^{-1}$ between the radial velocity of the star at $-7.1 \mathrm{~km} \mathrm{~s}^{-1}$ and the velocity of the $\mathrm{G}$ Colorado cloud at $-17.21 \mathrm{~km} \mathrm{~s}^{-1}$. The F0 V field star HD 147787, which is located at a distance of $40 \mathrm{pc}$ and a projected separation of $\sim 4^{\circ}$ from HD 145689, does not show any non-photospheric features in its spectrum. Thus, given this fact and since the difference between the $\mathrm{Ca}$ II feature and the stellar $v_{\text {rad }}$ is $\sim 2 \sigma$, we cautiously assign a CS origin to the Ca II absorption.

- HD 158352 (HR 6507) has two Ca II H\&K and Na I D absorption components that are relatively close to the stellar $v_{\text {rad }}$. 
There are no Colorado clouds along the line of sight of the star, but it passes near $\left(<20^{\circ}\right)$ several clouds with similar velocity vectors. Our spectra reveal a faint narrow Ca II $8542 \AA$ absorption as well as several faint Fe II and Ti II lines that are characteristics of shell stars. Iglesias et al. (2018) assigned the Ca II H\&K absorptions an ISM origin based on two field stars, HD 156208 and V $2373 \mathrm{Oph}$, that have absorptions similar to those of HD 158352. We find, however, that the ISM origin is ambiguous. HD 156208 and V 2373 Oph are at distances $\sim 217 \mathrm{pc}$ and $\sim 476 \mathrm{pc}$, respectively, that is, they are considerably further than the distance to HD 158352 of $\sim 63 \mathrm{pc}$. Therefore, while an ISM cloud could be located closer than $63 \mathrm{pc}$, it cannot be excluded that some ISM clouds responsible for the $\mathrm{Ca}$ II $\mathrm{H} \& \mathrm{~K}$ and $\mathrm{Na}$ I D are located between HD 158352 and the other two stars. On the other hand, Welsh \& Montgomery (2015) observed two Ca II K absorption components and attribute one of them as having a CS origin since it coincides with the stellar $v_{\text {rad }}$. Thus, while CS gas is certainly present around the star, the origin of the Ca II H\&K and $\mathrm{Na}$ I $\mathrm{D}$ absorptions is not completely obvious. We note that Lagrange et al. (2009) identified this star as a binary candidate.

- HD 168646 (HR 6864) has a pronounced triangular profile in the Ca II at the bottom of the stellar lines as well as many strong shell lines of Ti II, Fe II, and strong cores in the Balmer lines. The $\mathrm{Na}$ I $\mathrm{D}$ lines present a strong feature close to the $\mathrm{Ca}$ II one and a weaker one at a velocity of $\sim-22 \mathrm{~km} \mathrm{~s}^{-1}$ away from the Aql cloud. A very weak third component might only appear in the D2 line at a velocity of $\sim 11 \mathrm{~km} \mathrm{~s}^{-1}$. If this is real, this weak feature seems to have small variations in flux; we cannot make, however, any sound statement as our spectra are from a single epoch. All features have velocities that are far away from all ISM Colorado clouds. In any case, the strong shell spectrum clearly traces the presence of CS gas.

- HD 177724 ( $\zeta$ Aql) and HD 210418 have weak Ca II nonphotospheric absorptions; each is at its respective stellar radial velocity and close to some Colorado clouds. We did not find any useful field star in the ESO archive that could help to elucidate the origin of the features. Thus, the ascription to any CS or ISM origin is ambiguous.

- HD 196724 is a candidate shell star (Hauck \& Jaschek 2000) with low rotational velocity, which shows a weak Ca II absorption at the stellar $v_{\text {rad }}$ and is also close to the $v_{\text {ISM }}$ of the Mic and Aql clouds. It might have weak $\mathrm{Na}$ I absorption, but the spectra are too noisy to make a firm conclusion. The weak Ca II feature does not reveal a sharp, pronounced profile as it is seen in the $\mathrm{Ca}$ II K\&H lines in other shell stars. Thus, we find the origin of the $\mathrm{Ca}$ II (and $\mathrm{Na}$ I if real) absorptions to be ambiguous.

- HD 198160 (HR 7959) is a $\lambda$ Bootis type star with a very weak $\mathrm{Ca}$ II $\mathrm{K}$ absorption at the stellar $v_{\text {rad }}$ and the Vel cloud $v_{\text {ISM }}$. It forms a binary system with another $\lambda$ Bootis star, HD 198161 (Holweger \& Rentzsch-Holm 1995; Faraggiana $\&$ Bonifacio 1999); both components have a projected angular separation of just 2'.3. Weak Ca II $\mathrm{K}$ and $\mathrm{Na}$ I absorptions were detected towards HD 198161. We also note that a very weak $\mathrm{Na}$ I absorption is present towards HD 198160. Thus, while a CS origin cannot be excluded, we find an ISM origin to be more realistic given the fact that we detected very similar absorptions towards both stars. However, we note that Holweger \& RentzschHolm (1995) and Holweger et al. (1999) favour a CS origin around the binary.

- Stars belonging to the Upper Scorpius sub-group in the Scorpius-Centaurus association deserve particular attention. There are 13 stars in our sample that belong to this subgroup (Table B.1). All of them are located in a region of 5 sq. deg. and are between 110 and $150 \mathrm{pc}$. The stars show

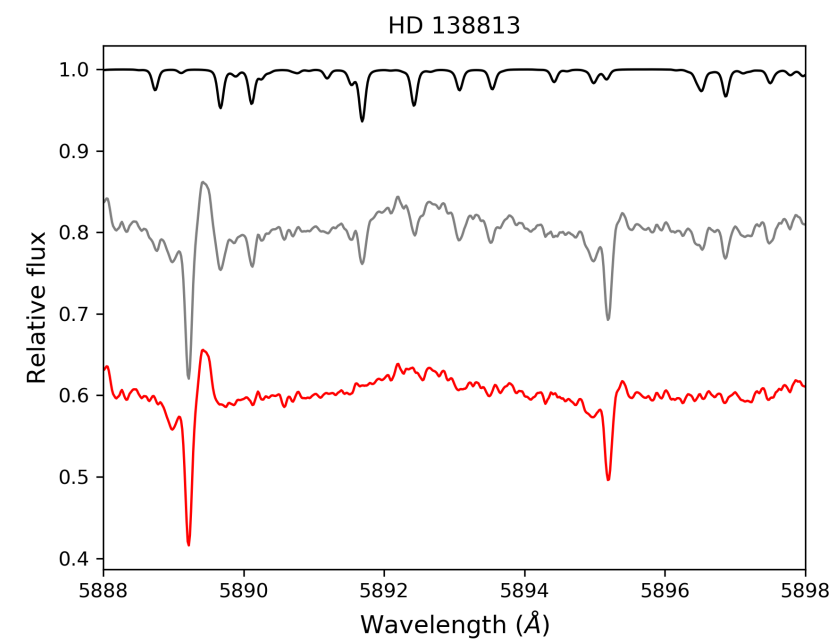

Fig. 4. Na I D lines of HD 138813, where the emission near the radial velocity of the star is easily recognisable and clearly present in the uncorrected spectra (grey line) and does not originate as an over subtraction of the atmospheric model (black line). The red line shows the telluric corrected spectrum.

non-photospheric $\mathrm{Ca}$ II and $\mathrm{Na}$ I absorption components, which tend to be grouped around $\sim-9 \mathrm{~km} \mathrm{~s}^{-1}, \sim-15 /-23 \mathrm{~km} \mathrm{~s}^{-1}$, and $\sim-28 \mathrm{~km} \mathrm{~s}^{-1}$ (Table B.3), which is in agreement with Welty et al. (1994). Most of the stars, with a few exceptions, have two features with similar velocities in both $\mathrm{Ca}$ II and $\mathrm{Na}$ I lines. Depending on the star, those two features are distributed among the three mentioned velocity ranges. The more blue-shifted components at $\sim-28 \mathrm{~km} \mathrm{~s}^{-1}$ are close to the $\mathrm{G}$ cloud velocity vector; the least blue-shifted components at $\sim-9 \mathrm{~km} \mathrm{~s}^{-1}$ are often close to the radial velocities of the stars. The fact that similar features, including those in the range $\sim-15 /-23 \mathrm{~km} \mathrm{~s}$, are shared in one way or another by all stars strongly suggests that they are of ISM origin, as already noticed by Rebollido et al. (2018) for HD 138813 and HD 146897. In general, the strongest feature is the less blue-shifted one, that is, the one close to the stars' $v_{\text {rad }}$; this trend holds irrespectively of whether one or two absorption features are detected in any of the $\mathrm{Ca}$ II and/or $\mathrm{Na}$ I. However, the exceptions to this general trend are HD 146606 and HD 145964, which we refer to again in Sect. 4.2 as it has a non-photospheric event identified by Welsh \& Montgomery (2013). The above mentioned results clearly point out that the ISM towards Upper Scorpius is not homogeneous, but it has a notorious complexity that is likely structured in clumps or relatively small cloudlets, with different properties, that are located at different distances, which are discernible along very nearby lines of sight. Finally, we note that a faint emission feature at $\sim-1.2 \mathrm{~km} \mathrm{~s}^{-1}$ was detected in both lines of the Na I doublet towards HD 138813. As shown in Fig. 4, the emission is not related to the telluric subtraction. The emission feature is at the radial velocity of the star and it is similar to the emission feature detected towards HD 156623, a star with Ca II variable events (Rebollido et al. 2018, 2019). These emissions most likely originate in the CS medium as they are observed in every spectra, regardless of the observing campaign or atmospheric conditions.

\subsection{Variable gas detection}

Sixteen stars in our sample were selected because of variable, $\beta$ Pic-like events of their Ca II H\&K and/or Na I D lines. The stars HD 56537 ( $\lambda$ Gem), HD 108767 ( $\delta$ Crv), HD 109573 (HR 

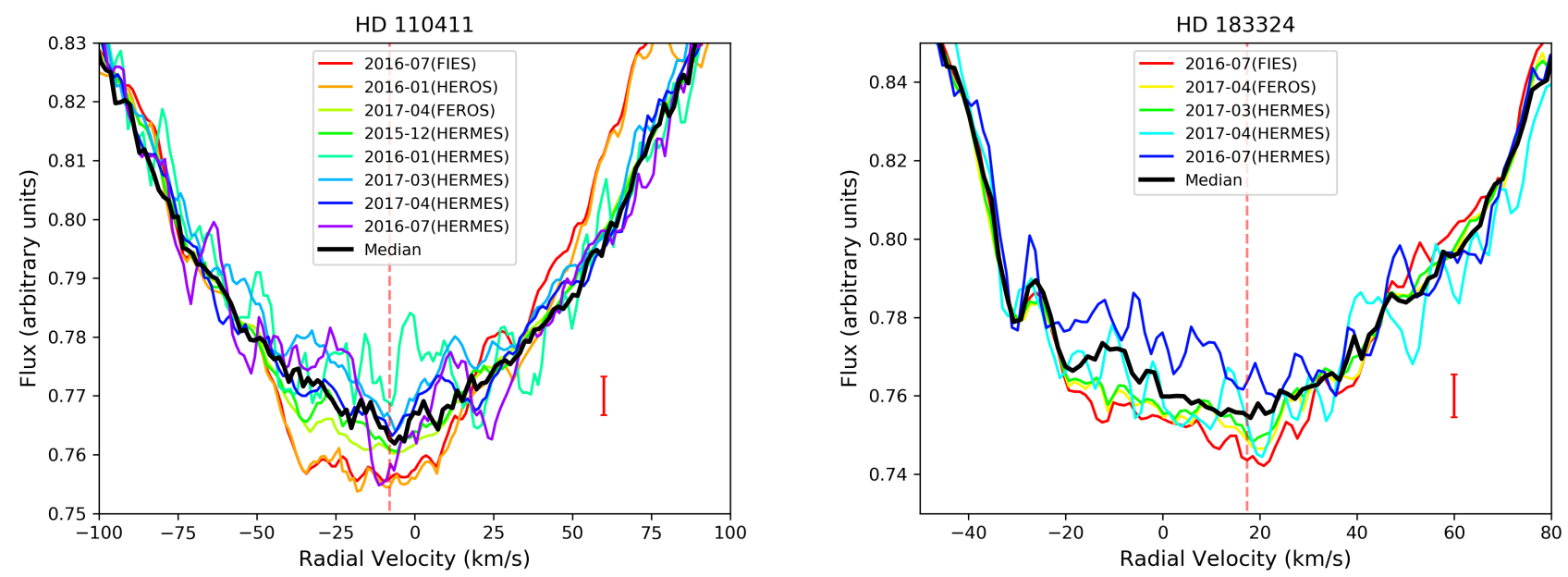

Fig. 5. Photospheric profile of Ca II K spectra of HD 110411 and HD 183324. The dates and instruments are colour-coded as indicated in the legend. The core of the line varies, although neither a narrow feature nor transient events are clearly distinguishable. The red-dashed vertical line marks the radial velocity of the star. The red error bar shows the 3- $\sigma$ standard deviation in the region close to the bottom of the line. This applies to all upcoming figures.

4796), and HD 148283 (HR 6123) also present optical (or UV) events (e.g. Grady et al. 1996b; Welsh \& Montgomery 2015; Iglesias et al. 2018), although they were included on the basis of other criteria (Table B.1). These stars are listed in Table 2 together with the following five new stars showing variability in non-photospheric features found in the frame of this work: HD 36546, HD 37306, HD 39182 (HR 2025), HD 98058 ( $\phi$ Leo), and HD 156623 (HIP 84881). We also include, in Table 2, the star HD 132200 even though this star was not included in our sample and has not been directly observed by us. A variable Ca II K absorption feature was found by Rebollido et al. (2018). Our results on $\phi$ Leo, HD 156623, and HR 10 have already been discussed in Eiroa et al. (2016), Rebollido et al. (2018, 2019), and Montesinos et al. (2019), respectively.

\section{Comments on individual stars}

- HD 9672 (49 Cet), HD 32297, HD 50241, HD 56537 ( $\lambda$ Gem), HD 64145 ( $\phi$ Gem), HD 108767 ( $\delta$ Crv), and HD 148283 (HR 6123) do not present any apparent transient event in our time series spectra (Table 2). Also, while we do not see any variability in HD 138629 (HR 5774), our spectra differ from previous ones (see below). Furthermore, we note that the stars HD 56537, HD 64145, HD 110411, and HD 183324 do not present any stable, narrow absorption at the core of the photospheric line, although HD 110411 and HD 183324 seem to present variability at the bottom of the Ca II K line (Fig. 5, see also Iglesias et al. 2018).

- HD 21620 presents one stable non-photospheric absorption in both $\mathrm{Ca}$ II and $\mathrm{Na}$ I at $\sim 4 \mathrm{~km} \mathrm{~s}^{-1}$ (Fig. A.1 and Table B.3). This feature does not coincide with either the radial velocity of the star or any of the Colorado ISM clouds; it has, however, a plausible ISM origin as several neighbouring stars have similar Na I absorptions (Génova \& Beckman 2003; Welsh \& Montgomery 2013). A weak absorption detected in Ca II K at $\sim 16 \mathrm{~km} \mathrm{~s}^{-1}$ is close to the ISM velocity of the LIC cloud. Nonetheless, the variability of this feature, which is also noted by Welsh \& Montgomery (2013, see their Figs. 1 and 2), is remarkable and we attribute it to a CS origin. Figure 6 shows the Ca II K line of HD 21620 during the campaigns of 2015 and 2016. We detected mostly red-shifted variations in the range of $\sim 10-30 \mathrm{~km} \mathrm{~s}^{-1}$, with a tentative, weak blue-shifted event on September 4-5, 2015 at $\sim 0 \mathrm{~km} \mathrm{~s}^{-1}$ (top left panel of Fig. 6), and a

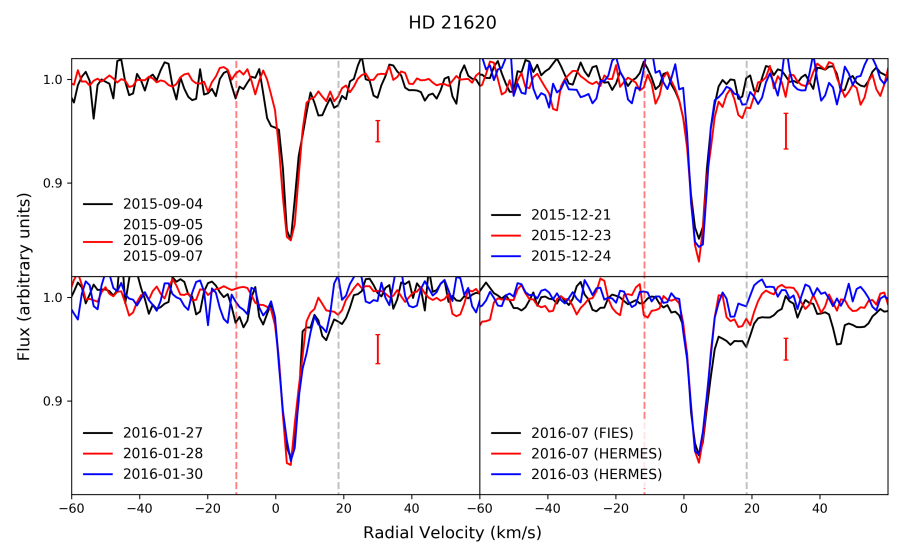

Fig. 6. Ca II K line of different epochs of HD 21620. The spectra that are plotted in the panels at the top and lower left were obtained with HERMES. Lower right panel: median spectra of the indicated period and telescope. FEB-like events appear at $\sim 16 \mathrm{~km} \mathrm{~s}^{-1}$ and tentatively at zero (blue-shifted, top left panel) and $50 \mathrm{~km} \mathrm{~s}^{-1}$ (red-shifted, bottom right). The events of January 2016 appear to present some dynamical evolution. Vertical red-and grey-dashed lines show the stellar and ISM radial velocities, respectively.

potential red-shifted event at $\sim 50 \mathrm{~km} \mathrm{~s}^{-1}$ in the NOT median spectra of July 2016 (bottom left panel of Fig. 6). We note that Welsh \& Montgomery (2013) also detected a feature at a velocity close to this last event together with several blue-shifted ones. Some dynamical evolution might be traced by the $\sim 16 \mathrm{~km} \mathrm{~s}^{-1}$ events observed in January 2016 as suggested by their changes in velocity and depth during three consecutive nights. All of the $\mathrm{Ca}$ II $\mathrm{K}$ events that we detected are very weak with no detectable counterpart in the $\mathrm{Ca}$ II $\mathrm{H}$ line, which suggests that the gas is optically thin. As an example, Fig. 7 shows both Ca II lines as observed on Dec 23, 2015. The K line can be fitted with two Gaussians at velocities 4.3 and $16.8 \mathrm{~km} \mathrm{~s}^{-1}$ as well as equivalent widths of 14.2 and $3.3 \mathrm{~m} \AA$, respectively. At the same time, the $4.3 \mathrm{~km} \mathrm{~s}^{-1} \mathrm{H}$ absorption, which is the stable one, has an $E W=7.2 \mathrm{~m} \AA$, that is, this feature is optically thin, while the $16.8 \mathrm{~km} \mathrm{~s}^{-1}$ variable one is embedded in the noise, also suggesting optically thin gas. 


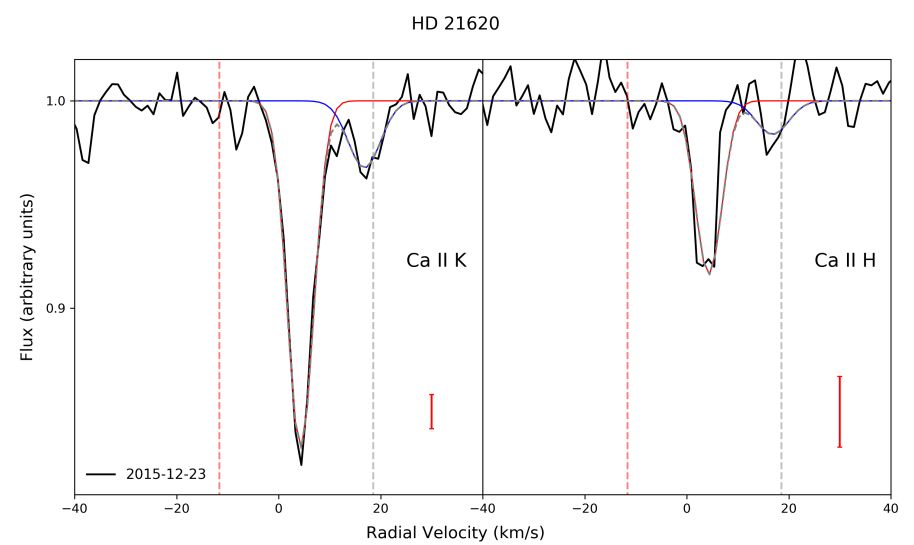

Fig. 7. Ca II K and H lines as observed on December 23, 2015 using HERMES. Two Gaussians are fitted to the $\mathrm{K}$ non-photospheric feature with velocities of $4.3 \mathrm{~km} \mathrm{~s}^{-1}$ (red continuous line) and $16.8 \mathrm{~km} \mathrm{~s}^{-1}$ (blue continuous line). The strongest, stable $4.3 \mathrm{~km} \mathrm{~s}^{-1}$ absorption is clearly detected in the $\mathrm{H}$ line, but the weakest, variable absorption at $16.8 \mathrm{~km} \mathrm{~s}^{-1}$ is embedded in the noise of the $\mathrm{H}$ spectrum. Vertical red- and grey-dashed lines show the stellar and ISM radial velocities, respectively.

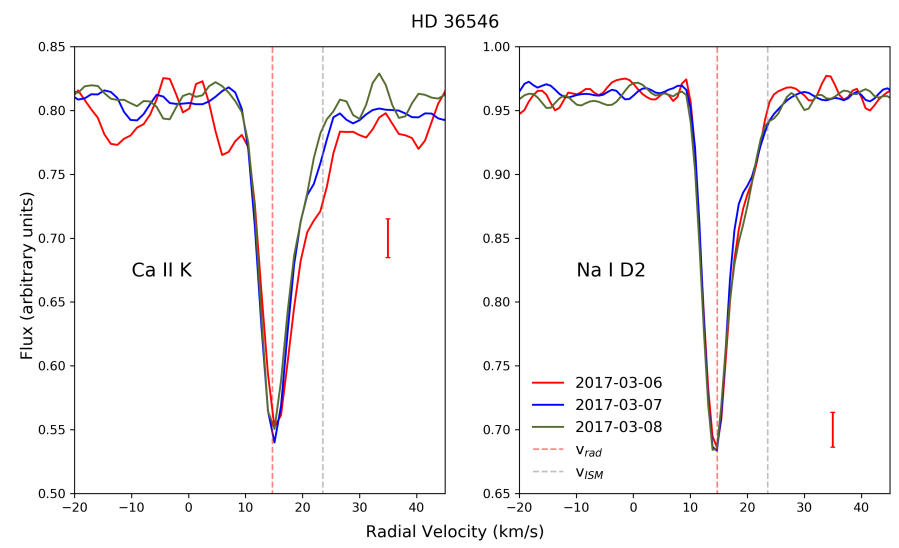

Fig. 8. Left panel: Ca II $\mathrm{K}$ line. A transient event is seen at $\sim 20 \mathrm{~km} \mathrm{~s}^{-1}$, which is superimposed on the red wing of the narrow non-photospheric absorption at $\sim 15 \mathrm{~km} \mathrm{~s}^{-1}$. No obvious event is seen on March 8, 2017. Right panel: Na I D2 line. Dates are as indicated and all spectra were obtained using HERMES. The red and grey vertical lines mark the radial velocity of the star and of the ISM, respectively.

- HD 36546 presents a narrow feature at a velocity of $\sim 15 \mathrm{~km} \mathrm{~s}^{-1}$, which is visible in both $\mathrm{Ca}$ II and $\mathrm{Na}$ I lines (Fig. A.1). The origin of the feature is most likely CS as it coincides with the radial velocity of the star and is far from the ISM cloud along the line of sight (Table B.3 and Fig. A.1). A redshifted event at a velocity of $\sim 20 \mathrm{~km} \mathrm{~s}^{-1}$ was detected in the Ca II K spectrum from March 6, 2017, which apparently evolved in the following dates and practically disappeared in the spectrum of March 8, 2017 (Fig. 8, left panel). A Gaussian deconvolution from the March 6, 2017 Ca II K absorption (Fig. 9) gives an EW of $8.4 \pm 0.9 \mathrm{~m} \AA$ for the $20 \mathrm{~km} \mathrm{~s}^{-1}$ event and $24.8 \pm 2.5 \mathrm{~m} \AA$ for the narrow stronger feature centred at $15 \mathrm{~km} \mathrm{~s}^{-1}$, which is similar to the EW of $27.1 \pm 2.7 \mathrm{~m} \AA$ of this narrow feature in the spectrum of March 8, 2017. We note that the weak feature at $20 \mathrm{~km} \mathrm{~s}^{-1}$ is not discernible from the noise in the $\mathrm{Ca}$ II $\mathrm{H}$ line (not shown), suggesting that the gas is optically thin and somehow similar to the case of HD 21620. We also point out that the Na I D2 line

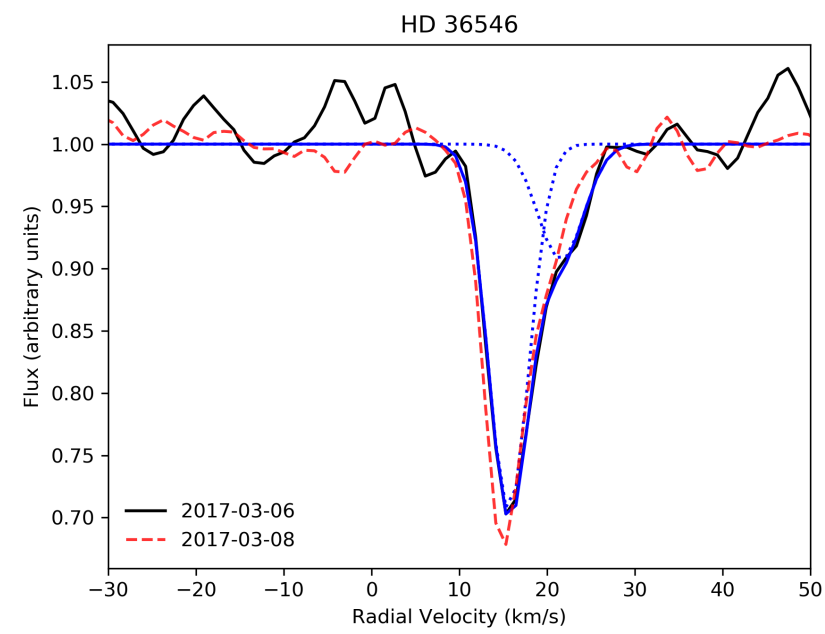

Fig. 9. Ca II K spectra of the HD 36546 non-photospheric absorption for the day where the event in the red wing is most conspicuous (March 6, 2016, black line) and when it is practically undetectable (March 8, 2016, red-dashed line). As in the previous figure, both spectra were obtained with HERMES. The blue solid line shows a fit of the March 6, 2016 spectrum with two Gaussians, each one is plotted as blue-dotted lines.

also presents an asymmetry in the red wing, with a small change of slope when comparing the different dates (Fig. 8, right panel).

- HD 36546 hosts a bright debris disc (Table B.1), which is seen near edge-on with an inclination angle $i \sim 70-75^{\circ}$ (Currie et al. 2017), following the trend suggested by Rebollido et al. (2018) between the disc inclination and the presence of narrow non-photospheric absorptions at the radial velocity of the star. Lisse et al. (2017) found evidence of a C-rich CS environment, which makes HD 36546 similar to $\beta$ Pic and $49 \mathrm{Cet}$ (Roberge et al. 2006, 2014). Thus, it can be another example of an enhanced carbon abundance acting as a braking mechanism of the hot inner $(<1 \mathrm{AU}) \mathrm{CS}$ gas released by the evaporation of exocomets, dust grains, or grain-grain collisions (Fernández et al. 2006; Brandeker 2011).

- HD 37306 presents two stable narrow $\mathrm{Ca}$ II and $\mathrm{Na}$ I absorptions (Fig. A.1) at velocities $\sim 11 \mathrm{~km} \mathrm{~s}^{-1}$ and $\sim 32 \mathrm{~km} \mathrm{~s}^{-1}$, while the stellar $v_{\text {rad }}$ is $25.1 \mathrm{~km} \mathrm{~s}^{-1}$. Nonetheless, the most remarkable and striking behaviour is the strong shell-like spectrum that appeared on the spectra of September 2017. The Ca II H\&K lines developed a strong, symmetric, triangular profile that is superimposed on the photospheric lines and the two narrow interstellar features, together with narrow shell-like absorptions in the Ca II triplet or in several Fe II and Ti II lines (see Fig. 10). At the same time, photospheric lines, such as the $\mathrm{Mg}$ II $4481 \AA$ or the O I triplet at $7750 \AA$, remained constant as well as the $\mathrm{Na}$ I D lines. Furthermore, the strong shell spectrum fully vanished in additional spectra taken on November 28, 2018 with the CARMENES spectrograph at the Calar Alto Observatory (Quirrenbach et al. 2016) and from December 14-18, 2018 with HERMES. This behaviour was also observed by Iglesias et al. (2019) by partly using the same spectra. Due to the unusual nature of this phenomenon, we checked possible sources of contamination, such as instrumentation issues or additional sources in the fibre, but we have discarded these scenarios. Thus, the appearance and disappearance of the shell-like profiles does indicate the presence of CS gas, but no blue- or red-shifted FEB-like events were detected in any of our spectra. We note that variability of shell spectra and even its appearance and disappearance in some stars is well known (e.g. Jaschek et al. 1988). 


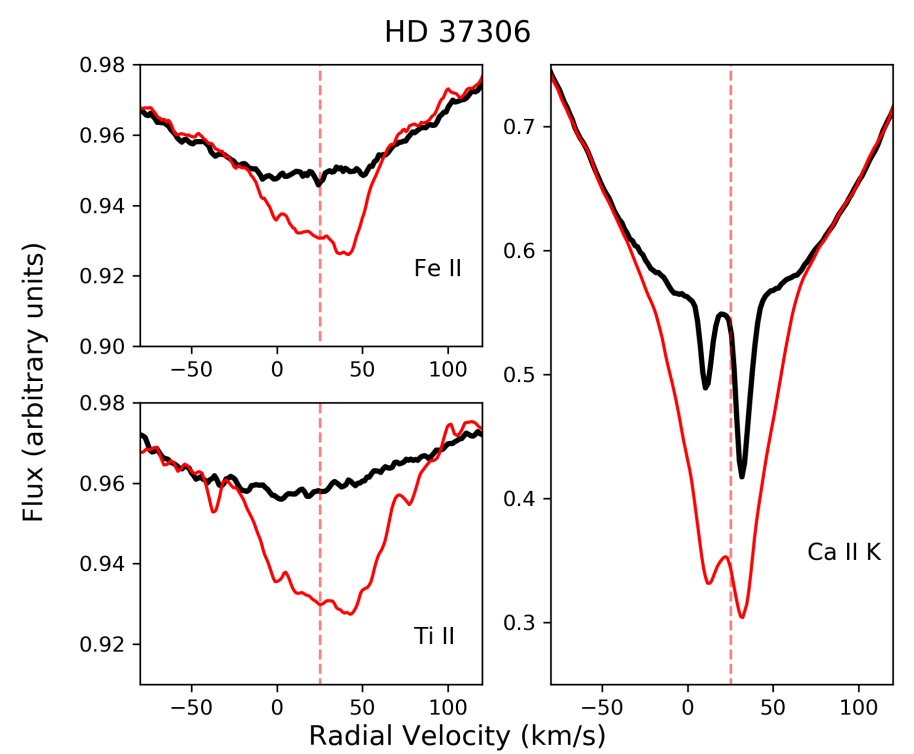

Fig. 10. Fe II (4583.83 $\AA$ ), Ti II (4443.80 ̊), and Ca II K lines of HD 37306. While most of the spectra, represented by the median spectrum in the plot (black line), do no vary and show two Ca II non-photospheric absorptions, a strong shell-like profile appeared in September 2017 (red line, FEROS). It was not observed again in later spectra in November and December 2018, which are not shown in this figure. The red vertical line marks the radial velocity of the star.

HD 39182

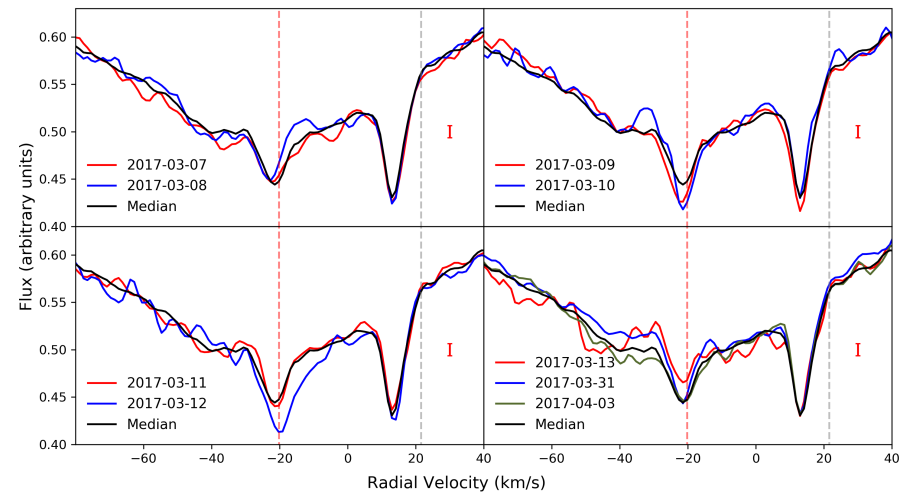

Fig. 11. Ca II K spectra of HD 39182 grouped by observing dates obtained with HERMES. In all panels, the median spectra is also plotted. The red and grey vertical lines correspond to the radial velocity of the star and the velocity vector of the LIC Colorado cloud, respectively.

At the same time, and despite the lack of any identified ISM clouds in the line of sight, the fact that no remarkable changes are seen in the mentioned $\sim 11 \mathrm{~km} \mathrm{~s}^{-1}$ and $\sim 32 \mathrm{~km} \mathrm{~s}^{-1}$ narrow features during the eight days of observations in September 2017, while drastic changes are seen in the CS (shell-type) environment, suggests an ISM origin as the more plausible alternative for those two absorptions, which is in agreement with Iglesias et al. (2018).

- HD 39182 (HR 2025), which is one of the selected Ti II stars, has a sharp triangular-like absorption (Fig. A.1) with two narrow Ca II components; one is found at $\sim-22 \mathrm{~km} \mathrm{~s}^{-1}$, which is centred on the photospheric line coinciding with the stellar $v_{\text {rad }}$, and varies significantly; the second and strongest one at $\sim 13 \mathrm{~km} \mathrm{~s}^{-1}$ is clearly displaced from the line centre (Fig. 11). A weak extra absorption at $\sim-41 \mathrm{~km} \mathrm{~s}^{-1}$ is present in some spectra, that is, in the blue wing of the $-22 \mathrm{~km} \mathrm{~s}^{-1}$ component

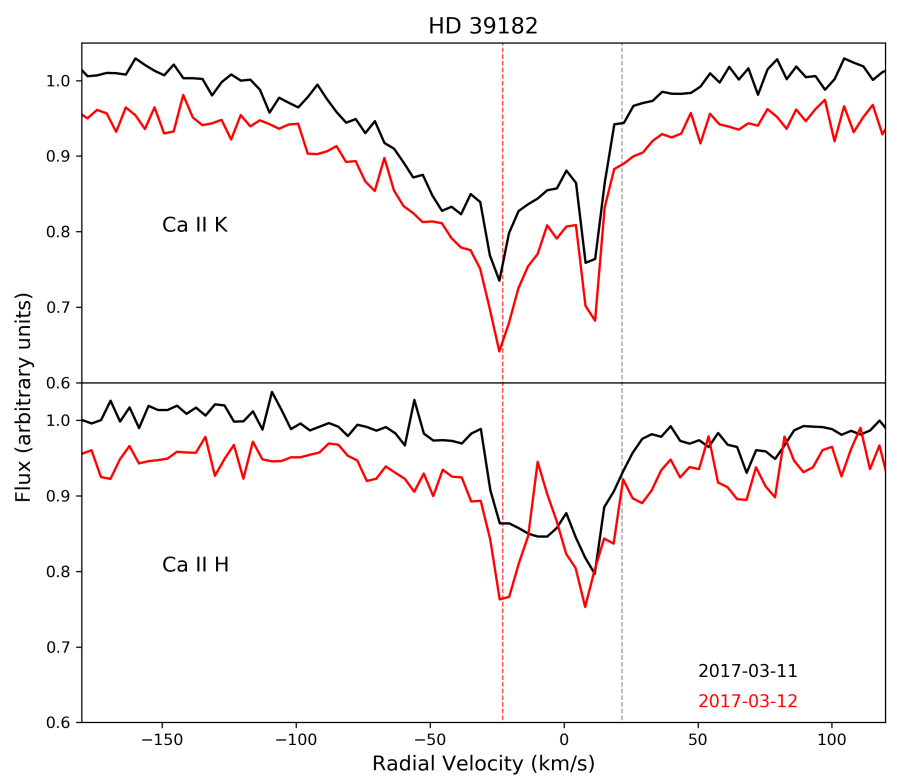

Fig. 12. Ca II K and H lines of HD 39182 are colour-coded for two different observing dates obtained with HERMES. Spectra were shifted 0.05 units in the Y-axis with the aim of facilitating the visualisation of the variability. The red and grey vertical lines correspond to the radial velocity of the star and the velocity vector of the LIC Colorado cloud, respectively.

(Figs. A.1 and 11). The Na I D lines only show a strong narrow absorption at $\sim 14 \mathrm{~km} \mathrm{~s}^{-1}$, coinciding with the strongest $\mathrm{Ca}$ II component. None of the velocity components coincide with the velocity vector $\left(v_{\mathrm{ISM}}=21.62 \mathrm{~km} \mathrm{~s}^{-1}\right)$ of the LIC cloud, which is seen along the line of sight to the star. As an example of the observed Ca II variability, Fig. 12 shows both $\mathrm{H}$ and $\mathrm{K}$ lines taken in two consecutive nights, where remarkable variations of the depth and profile of both non-photospheric features are observed. We also note that our spectra differ from the one reported by Lagrange-Henri et al. (1990b). Their spectrum shows a strong Ca II feature at the bottom of the stellar line, that is, it is similar to our $-22 \mathrm{~km} \mathrm{~s}^{-1}$ feature, but the strong $13 \mathrm{~km} \mathrm{~s}^{-1}$ absorption in our spectra, if present, is much weaker. Further, Lagrange-Henri et al. (1990b) did not report any Na I components. Thus, all of these results suggest that the origin of all absorptions are CS.

- HD 42111 (HR 2174) is a shell star with strong Ca II and $\mathrm{Na} \mathrm{I}$ absorptions in the median spectrum close to the radial velocity of the star and of the Aur cloud velocity (Fig. A.1 and Table B.3), which were previously reported by Lagrange-Henri et al. (1990b). Those authors attributed a CS origin (at least partially) to the Ca II absorption based on a comparison of the dispersion velocities (FWHMs) of Ca II K and Na I lines; this result was later confirmed by Lagrange-Henri et al. (1991) by comparing the non-photospheric features of HD 42111 and of the nearby star, HD 42092. Furthermore, the (at least partly) Ca II CS origin is corroborated by the fact that the EW and FWHM of the Ca II $\mathrm{K}$ absorption, as estimated with our spectra, are much larger that the ones reported in those works, while at the same time the EWs and FWHM of the strong Na I absorptions are similar; a small change in the $\mathrm{Ca}$ II $\mathrm{K}$ absorption was also pointed out by Welsh \& Montgomery (2013). Individual spectra of HD 42111 show that the Ca II $\mathrm{K}$ feature is formed by two components at velocities of $\sim 25 \mathrm{~km} \mathrm{~s}^{-1}$ and $27.5 \mathrm{~km} \mathrm{~s}^{-1}$, supporting the suggestion made by 


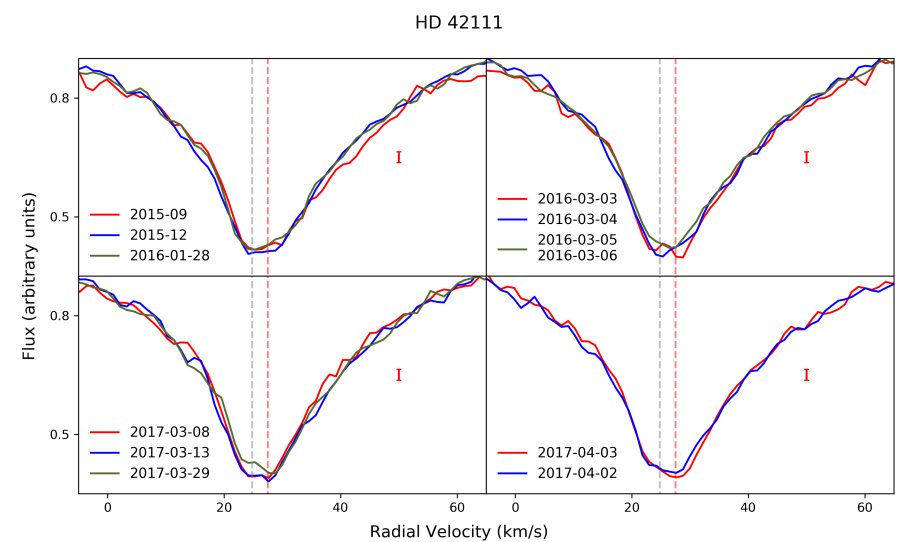

Fig. 13. Ca II K spectra of HD 42111 obtained with HERMES and grouped by observing dates. Spectra were shifted 0.05 units in the Yaxis. The red and grey vertical lines correspond to the radial velocity of the star and the velocity vector of the ISM in the line of sight.

Lagrange-Henri et al. (1990b) concerning the plausible blend of two distinct features. Both Ca II K components have a very similar strength (Fig. 13), although the feature at $\sim 25 \mathrm{~km} \mathrm{~s}^{-1}$ varies in depth while the one at $\sim 27.5 \mathrm{~km} \mathrm{~s}^{-1}$ only appears distinctly in some selected dates as a kind of $\beta$ Pic-like event, for example on March 3, 2016 or March 8, 2017 (Fig. 13), which likely has a small dynamical evolution, at least in the March 2016 spectra, (see top-right panel of Fig. 13). In this respect, we note that Welsh \& Montgomery (2013) report a FEB-like Ca II K event at $75 \mathrm{~km} \mathrm{~s}^{-1}$ and that Grady et al. (1996b) and Lecavelier Des Etangs et al. (1997) detected gas in UV lines of Fe II, Mn II, and $\mathrm{Mg}$ II, which were interpreted as CS clumps falling onto the star. With respect to the $\mathrm{Ca}$ II $\mathrm{H}$ feature, our individual spectra do not resolve both $\mathrm{K}$ components, and they are all well represented by their median profile; also, the peak of the $\mathrm{H}$ line feature is slightly red-shifted with respect to the $\mathrm{K}$ absorption. As an example, Fig. 14 plots the $\mathrm{Ca}$ II $\mathrm{H}$ and $\mathrm{K}$ lines of the two consecutive nights where that behaviour can be seen. This could be due to the fact that the broad Ca II $\mathrm{H}$ feature is severely blended with the strong triangular-like profile at the core of the Balmer $\mathrm{H} \epsilon$ line; such strong triangular-like profiles are clearly present in all Balmer lines. Obviously, additional higher resolution spectra are needed in order to attempt to resolve the $\mathrm{Ca}$ II $\mathrm{H}$ absorption without interference from the $\mathrm{H} \epsilon$ line and to study its plausible variability. We further note that a weak, but very broad, absorption is observed in both $\mathrm{Na} 1 \mathrm{D}$ lines producing the observed secondary peak (Fig. A.1). The absorption present in all individual and the median spectrum of HD 42111 is not evident in the spectra by Lagrange-Henri et al. (1990b, 1991), or the highresolution, unpublished spectrum obtained by EXPORT (Mora et al. 2001).

- HD 80007 has very weak Ca II K and Na I D2 absorptions at the stellar $v_{\text {rad }}$ (Table B.3 and Fig. A.1). The corresponding Ca II $\mathrm{H}$ and $\mathrm{Na}$ I D1 might be present in our median spectrum, but at the noise level (Fig. A.1), and new spectra are required before a sound confirmation can be made. Hempel \& Schmitt (2003) noticed a change in the equivalent width, shape, and velocity of the Ca II absorption. Redfield et al. (2007) also found variability in the velocity while the column density of the Ca II absorption remains relatively constant; in contrast, those authors found more remarkable variability in the velocity (two absorptions at $\sim-7 \mathrm{~km} \mathrm{~s}^{-1}$ and $\sim 7 \mathrm{~km} \mathrm{~s}^{-1}$ ) and column densities of the Na I feature. Further, Welsh \& Montgomery (2015) found a quasi two-

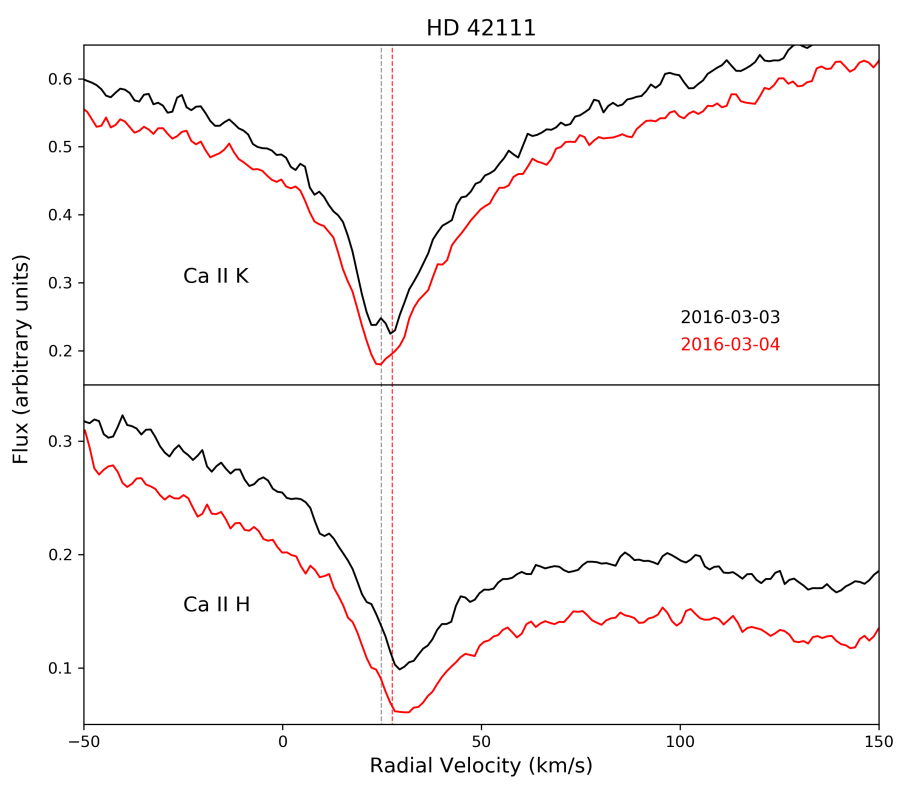

Fig. 14. Ca II H\&K spectra of HD 42111 obtained with HERMES and grouped as observed in the indicated consecutive dates. The photospheric contribution was not removed from this plot. The vertical lines correspond to the radial velocity of the star and the velocity vector of the ISM in the line of sight.

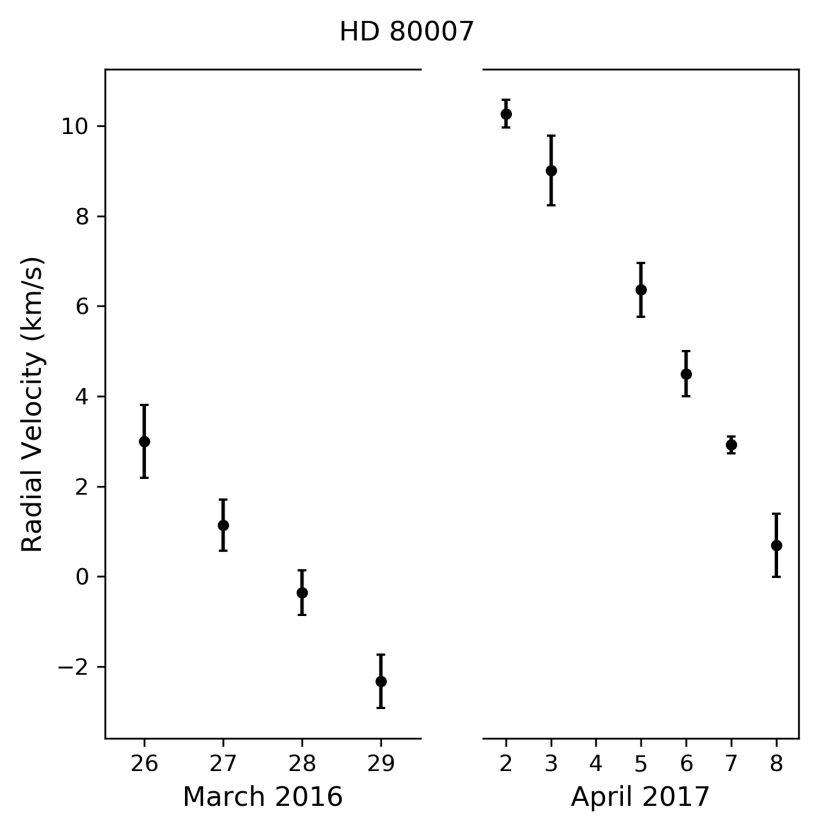

Fig. 15. Radial velocity variation of HD 80007 in both observing periods. The observations suggest the presence of an unseen companion.

component $\mathrm{Ca}$ II $\mathrm{K}$ feature in two consecutive nights and one single-component absorption in two other nights, with changes in the equivalent width. In addition, Wood \& Hollis (1971) found a quasi-periodic oscillation in the strength of the $\mathrm{H} \beta$ Balmer line and suggested it could be due to flares generated by acoustic oscillations in the stellar atmosphere.

Our spectra show new aspects of both $\mathrm{Ca}$ II and $\mathrm{Na} 1$ absorptions as well as in the stellar radial velocity. Firstly, the radial velocity of the star shows a regular variation of the order of $\sim 1.5 \mathrm{~km} \mathrm{~s}^{-1}$ per day in both 2016 and 2017 observing periods (Fig. 15). A possibility is that HD 80007 is a binary system 


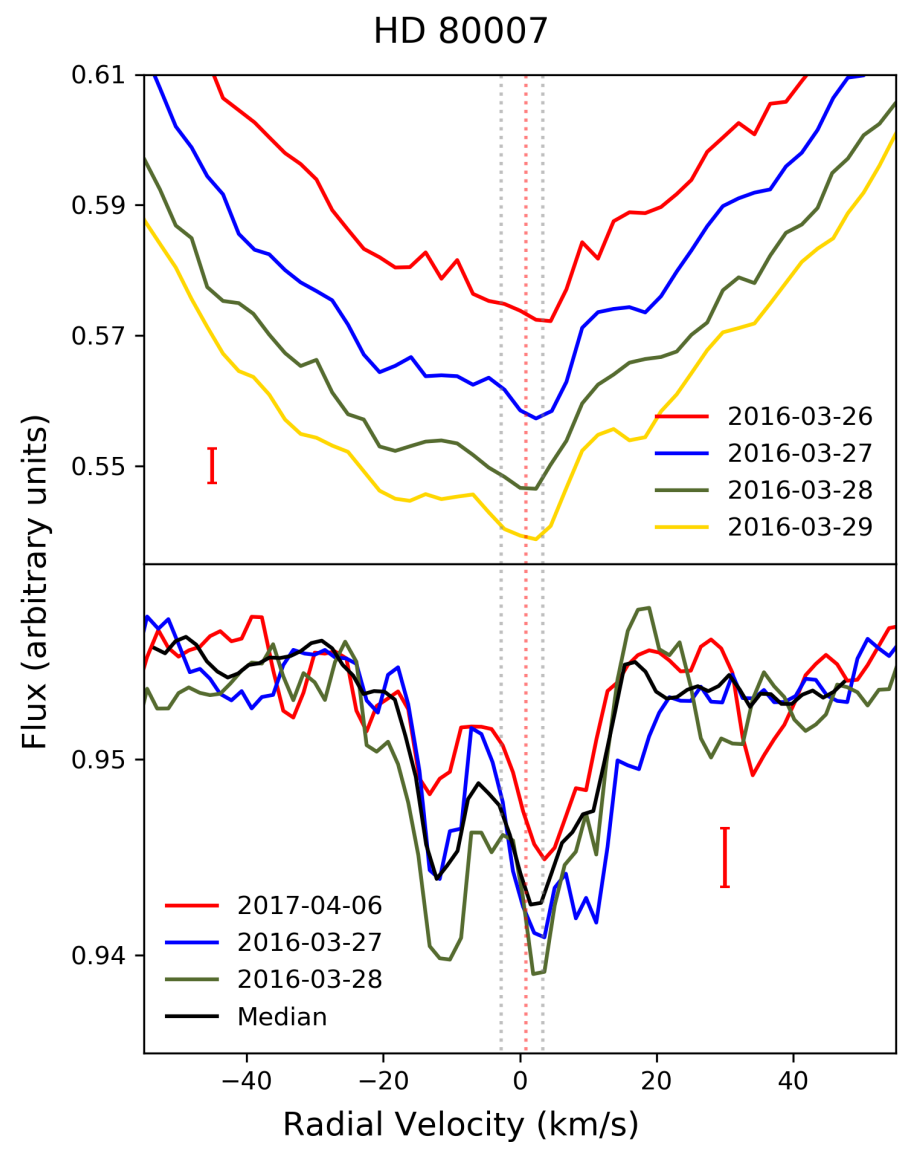

Fig. 16. Top panel: Ca II K line of HD 80007 for the selected days, observed with FEROS. Spectra were shifted 0.01 units in the Y-axis aimed at facilitating the visualisation of the variability. The central absorption is seen at $\sim 2 \mathrm{~km} \mathrm{~s}^{-1}$ as well as blue- and red-shifted variable absorptions. Bottom panel: Na I D2 line of HD 80007 for the selected days. Variability is seen at $\sim-10$ and $\sim 2 \mathrm{~km} \mathrm{~s}^{-1}$. The slight shift at the bottom of the narrow absorptions, which is presumably produced by a companion, is noticeable in both panels.

and that the radial velocity variability is induced by an unseen companion. Secondly, the Ca II K absorption shows a "central" feature with small changes in its strength accompanied in some spectra with blue- and red-shifted components (Fig. 16, top panel). At the same time, the Na I D2 feature presents two components. One is a broad, variable feature centred at $\sim 2 \mathrm{~km} \mathrm{~s}^{-1}$, that is, the velocity of the star and the Ca II feature, with a red-shifted wing up to $\sim 18.5 \mathrm{~km} \mathrm{~s}^{-1}$ that is even discernible in the median spectrum (Fig. 16, bottom panel). Furthermore, one red-shifted event at $\sim 10 \mathrm{~km} \mathrm{~s}^{-1}$ and extending up to $\sim 22 \mathrm{~km} \mathrm{~s}^{-1}$ might tentatively be present in the spectrum of March 27, 2016. The second Na I D2 component at a velocity of $\sim-11.5 \mathrm{~km} \mathrm{~s}^{-1}$ appears in all spectra but its depth varies. We note that the velocity difference between both features is approximately the same as the ones sporadically observed by Redfield et al. (2007).

- HD 85905 is a shell star whose median spectrum shows a sharp triangular-like $\mathrm{Ca}$ II absorption with two components at velocities at $\sim 8.4 \mathrm{~km} \mathrm{~s}^{-1}$ and $\sim 25.0 \mathrm{~km} \mathrm{~s}^{-1}$ as well as one Na I feature at $\sim 8.1 \mathrm{~km} \mathrm{~s}^{-1}$. The feature at $\sim 8 \mathrm{~km} \mathrm{~s}^{-1}$ coincides with the radial velocity of the star and is also close to the velocity vector of the G cloud (Fig. A.1 and Table B.3). Nonetheless, individual spectra from the different epochs show remarkable variability; Fig. 17 shows some examples. In December 2015, both Ca II $\mathrm{K}$ components experienced noticeable variations, while the cor-

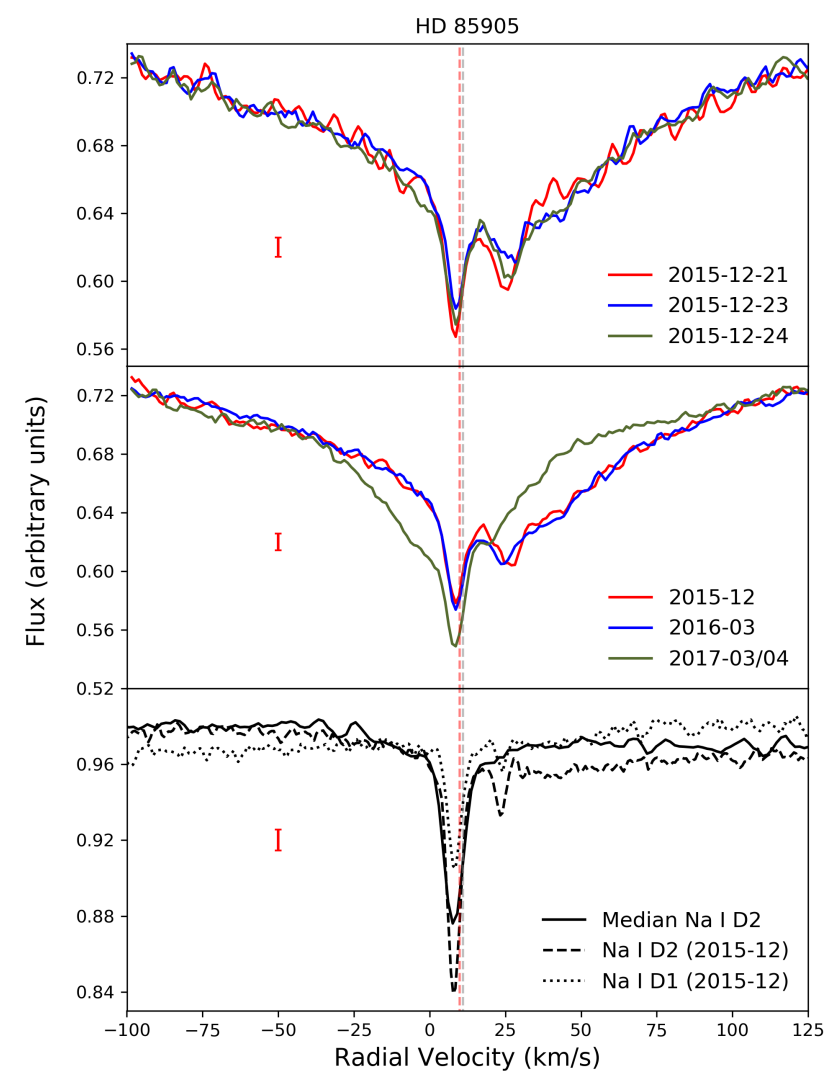

Fig. 17. Top panel: December 21, 23, and 24, 2015 where variability of the $\sim 25 \mathrm{~km} \mathrm{~s}^{-1} \mathrm{Ca}$ II $\mathrm{K}$ feature can be seen. Middle panel: Ca II K median spectra of three different campaigns using HERMES where the variability in the triangular profile is seen. The absorption at $\sim 25 \mathrm{~km} \mathrm{~s}^{-1}$ disappears in March 2017. Lower panel: Na I D lines of December 2015 where the absorption at $\sim 23 \mathrm{~km} \mathrm{~s}^{-1}$ is visible. The median of all spectra in the Na I D2 line where the absorption is no longer present is also shown. The vertical lines correspond to the radial velocities of the star and of the ISM.

responding $\mathrm{Na}$ I $8.0 \mathrm{~km} \mathrm{~s}^{-1}$ feature remained constant. However, there is a relatively strong feature at a velocity of $\sim 23.7 \mathrm{~km} \mathrm{~s}^{-1}$ in both $\mathrm{Na}$ I D lines (i.e. close to the Ca II $25.0 \mathrm{~km} \mathrm{~s}^{-1}$ component), which are visible in all dates of that period (December 2015) but not in any other of our observing epochs. The lower panel of Fig. 17 shows the median of December 2015 spectra of both Na I $\mathrm{D}$ lines where this result can be appreciated. Furthermore, while during the periods of December 2015, January 2016, and March 2016 both Ca II components were present, only the component at $\sim 8 \mathrm{~km} \mathrm{~s}^{-1}$ was visible during the two different campaigns of 2017 (March 8-11 and March 29 to April 8). During these campaigns, a strong absorption also appeared in the blue wing of the $\mathrm{Ca}$ II K, while the red-shifted $25.0 \mathrm{~km} \mathrm{~s}^{-1}$ feature practically disappeared. Figure 18 shows the profiles of both the Ca II H and $\mathrm{K}$ lines of the median spectra of the three previously mentioned periods where changes in the $\mathrm{H}$-line profile can also be appreciated. The above mentioned results clearly suggest a CS origin of the non-photospheric absorptions observed in HD 85905, which are potentially related to a variability in the CS shell as suggested by the variations observed in other shell lines of Fe II, for example, but not in photospheric lines as Mg II $4481 \AA$ or the O I triplet at $7775 \AA$ (not shown). A detailed analysis will be published elsewhere. Welsh et al. (1998) and Redfield et al. (2007) also attributed a CS origin to the Ca II and $\mathrm{Na}$ I absorptions they detected. 


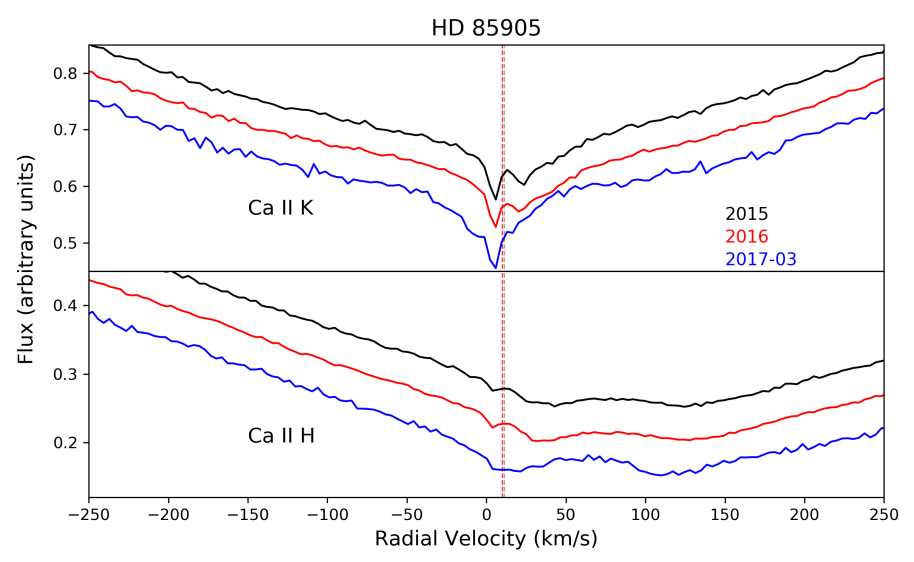

Fig. 18. Ca II $\mathrm{H}$ and $\mathrm{K}$ median spectra of the campaigns indicated in the labels obtained using HERMES, where the variability of both lines can be appreciated. Spectra were shifted 0.05 units in the Y-axis. The photospheric contribution was not removed in this plot. The vertical lines correspond to the radial velocities of the star and of the ISM.

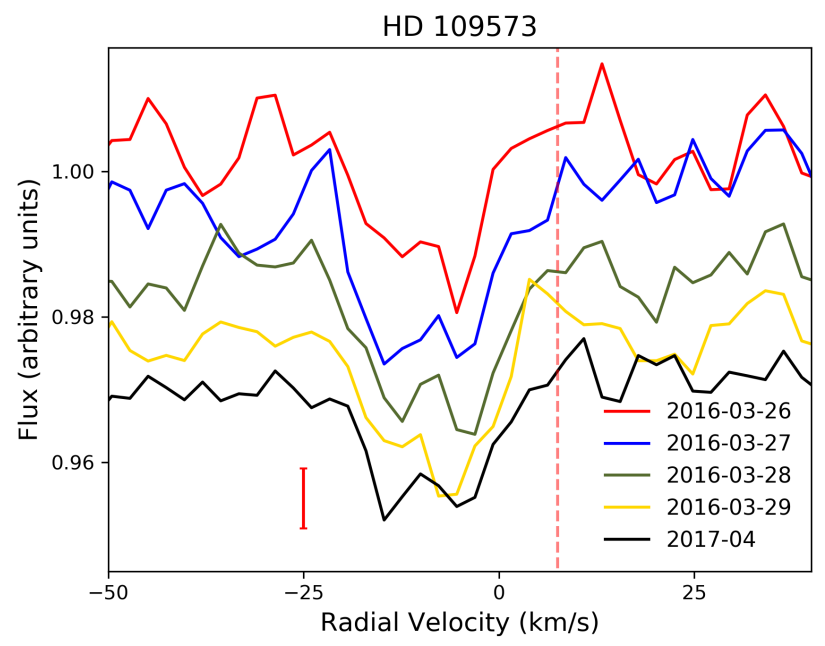

Fig. 19. Ca II K line of HD 109573. A shift of 0.005 was added to the Y-axis in order to help differentiate the variations. All spectra were obtained using FEROS. The red vertical line corresponds to the radial velocity of the star. The Ca II $\mathrm{K}$ line shows two absorptions, which are not coincident with the radial velocity of the star but with variations in their strength

- HD 109573 (HR 4796) has two very weak Ca II K absorptions at $\sim-14.2$ and $-4.7 \mathrm{~km} \mathrm{~s}^{-1}$ in the median spectrum (Fig. A.1). The one at $-14.2 \mathrm{~km} \mathrm{~s}^{-1}$ was not detected in the $\mathrm{Ca}$ II $\mathrm{H}$ line, suggesting the gas is optically thin, as it is the $-4.7 \mathrm{~km} \mathrm{~s}^{-1}$ feature. This latter absorption is also present in the $\mathrm{Na}$ I $\mathrm{D}$ lines. None of these features coincide with the radial velocity of the star. Figure 19 shows details of the Ca II K line on different dates (the $\mathrm{Ca}$ II $\mathrm{H}$ line is only revealed with the median spectra of all 23 individual spectra of HR 4796). Both Ca II K components vary in depth and shape and, in some cases, close to the noise level. Nonetheless, a discernible variability is seen, for example, the $-14.2 \mathrm{~km} \mathrm{~s}^{-1}$ component from March 26 and 29, 2016 is distinctively weaker than the one from March 27-28, 2016 or the median of April 2017. Our spectra and the variability of the $\mathrm{Ca}$ II $\mathrm{K}$ features are quite similar to those in Welsh \& Montgomery (2015), strongly suggesting a CS origin. Nonetheless, Iglesias et al. (2018) attribute the $-5 \mathrm{~km} \mathrm{~s}^{-1}$ feature to an ISM origin since the field star 1 Cen (HD 110073;

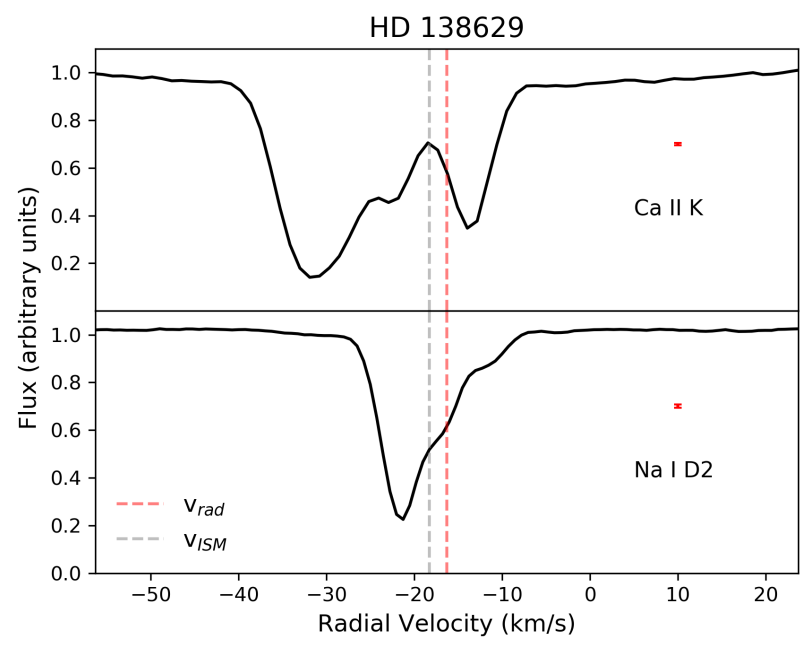

Fig. 20. Median HERMES spectra of $\mathrm{Ca}$ II $\mathrm{K}$ and $\mathrm{Na} 1 \mathrm{D} 2$ nonphotospheric absorption features observed towards HR 5774. The dashed lines correspond to the radial velocity of the star (red) and the velocity vector of the interstellar NGP cloud (blue).

30 pc behind HR 4796) also has a similar absorption feature. Welsh \& Montgomery (2015) detected a faint FEB-like event at $\sim 60 \mathrm{~km} \mathrm{~s}^{-1}$ in two spectra from a single night, and Iglesias et al. (2018) report a faint variable feature at the velocity of the star. None of these are apparent in our spectra.

- HD 138629 (HR 5774) has three non-stellar Ca II features at velocities $-31.8,-22.9$, and $-13.8 \mathrm{~km} \mathrm{~s}^{-1}$ with no sign of variability; none of them are clearly coincident with $v_{\text {rad }}$ or with $v_{\text {ISM }}$. The Na I D lines also present three absorptions; two of them coincide with two Ca II ones (Fig. 20). Our spectra differ from the two and four $\mathrm{Ca}$ II components as well as from one $\mathrm{Na}$ I feature, reported by Lagrange-Henri et al. (1990b). We attribute, at least in part, a CS origin due to the apparent changes with previous works, but an ISM origin cannot be excluded, at least for the features at $\sim-22 \mathrm{~km} \mathrm{~s}^{-1}$ and $\sim-12 \mathrm{~km} \mathrm{~s}^{-1}$.

- HD 145964 is one of the Upper Scorpius stars. Two absorption components at velocities of $\sim-9.5 \mathrm{~km} \mathrm{~s}^{-1}$ and $\sim-25 \mathrm{~km} \mathrm{~s}^{-1}$ were detected in $\mathrm{Ca}$ II and $\mathrm{Na}$ I lines (Fig. A.1 and Table B.3). Those components can be attributed to the ISM medium, in principle, as they were also detected in many other UpSco stars (see Sect. 4.1.2). However, while the profile remains practically constant for the Ca II K feature at $-9.0 \mathrm{~km} \mathrm{~s}^{-1}$, which is the velocity of the star, the asymmetric blue wing of the $-25 \mathrm{~km} \mathrm{~s}^{-1}$ absorption shows a weak $\sim-30 \mathrm{~km} \mathrm{~s}^{-1}$ component in most spectra of all observing runs, but not, for example, in both the Hermes and FEROS spectra, which were taken on the same dates in April 2017 (Fig. 21). We also note that a marginal variation in the relative depth of the $-9.0 \mathrm{~km} \mathrm{~s}^{-1}$ and $-25 \mathrm{~km} \mathrm{~s}^{-1}$ features might be present. The same behaviour is observed in the Ca II $\mathrm{H}$ line (Fig. 22).

Thus, a CS contribution is suggested, in particular for the $-25 \mathrm{~km} \mathrm{~s}^{-1}$ and $-30 \mathrm{~km} \mathrm{~s}^{-1}$ absorptions. Similar Ca II K absorptions were detected by Welsh \& Montgomery (2013), who also report a weak FEB-like event at a velocity of $\sim 50 \mathrm{~km} \mathrm{~s}^{-1}$ that is not detectable in our data.

- HD 172555 was already discussed by Rebollido et al. (2018) as one of the debris disc stars with both cold and hot gas in its circumstellar environment (see also the first paragraph of Sect. 4.1.2). Here we want to stress that a weak ISM feature was detected in both Ca II lines at a velocity of $\sim-20 \mathrm{~km} \mathrm{~s}^{-1}$, which is in good agreement with Kiefer et al. (2014b). In 


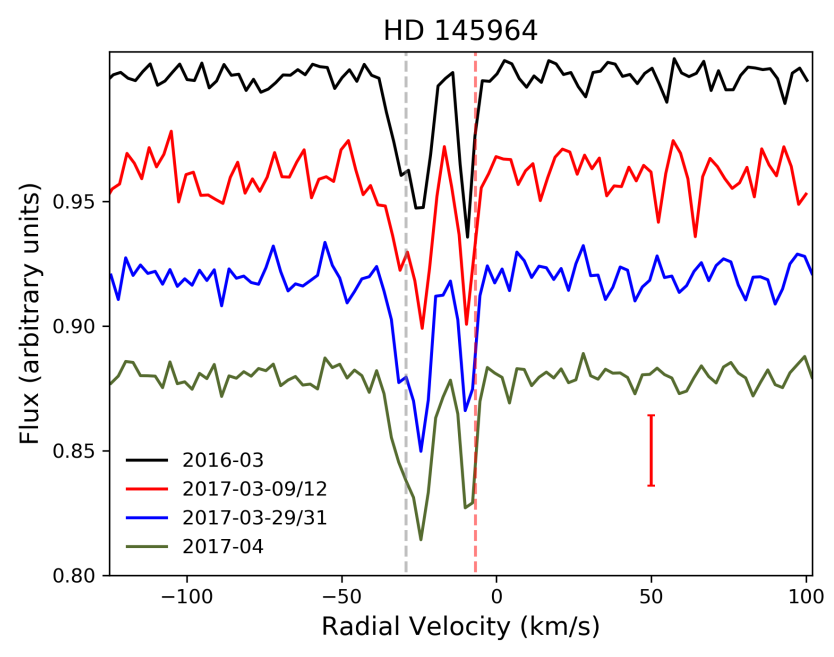

Fig. 21. Ca II K spectra of HD 145964 as indicated in the labels, which were obtained using HERMES. An offset of 0.04 was introduced in the Y-axis to better perceive the variations. The red and grey vertical lines correspond to the radial velocity of the star and the velocity vector of the G Colorado cloud, respectively.

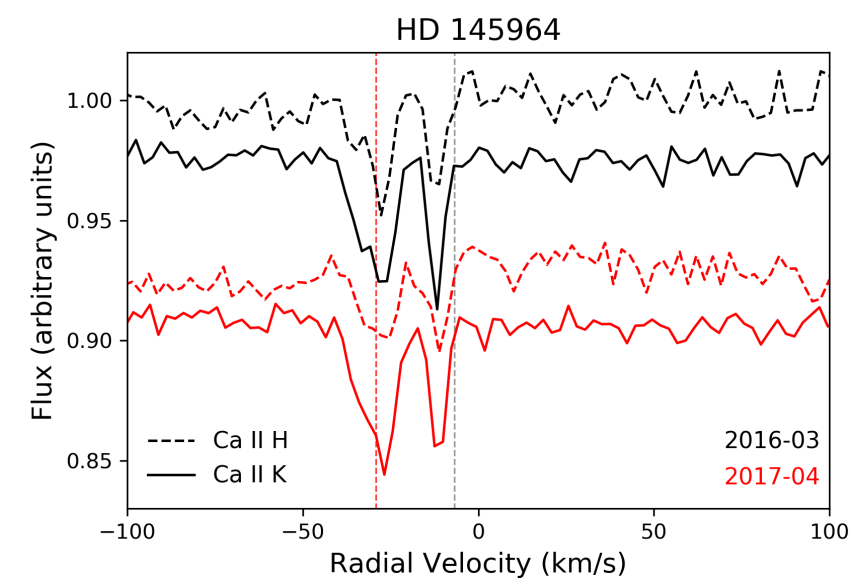

Fig. 22. Spectra of HD 145964 are colour-coded as indicated in the labels for Ca II K \& H lines (continuous and dashed lines, respectively) and were obtained with HERMES. An offset of 0.05 was introduced in the Y-axis to better perceive the variations between both dates, and 0.02 between the $\mathrm{K}$ and $\mathrm{H}$ lines. The red and grey vertical lines correspond to the radial velocity of the star and the velocity vector of the G Colorado cloud.

addition, our spectra reveal a weak, broad Na I D2 feature centred at $\sim 15.3 \mathrm{~km} \mathrm{~s}^{-1}$ and extending from $\sim-5 \mathrm{~km} \mathrm{~s}^{-1}$ up to $\sim 35 \mathrm{~km} \mathrm{~s}^{-1}$. Although the individual spectra are relatively noisy, we are confident about this detection as it appears in all spectra. The top panel of Fig. 23 shows the Na I D2 median spectrum and spectra from March 8, 2016 and April 8, 2017. The broad Na I D2 feature presents clear variability, denoting its CS nature. The bottom panel of Fig. 23 shows the telluric subtraction for the indicated dates, where it is clear that the variability is not related to telluric lines. Figure 23 (top panel) also shows the median of all Na I D1 line spectra, where the ISM feature is clearly visible but not the broad CS one. We note that Grady et al. (2018) detected some UV broad, variable absorptions of ions, such as CII.

- HD 182919 (5 Vul) has a weak, narrow absorption feature at $\sim-18.5 \mathrm{~km} \mathrm{~s}^{-1}$, which is close the velocity of the star and to the G, Mic, and Aql clouds (Table B.3 and Fig. A.1). The

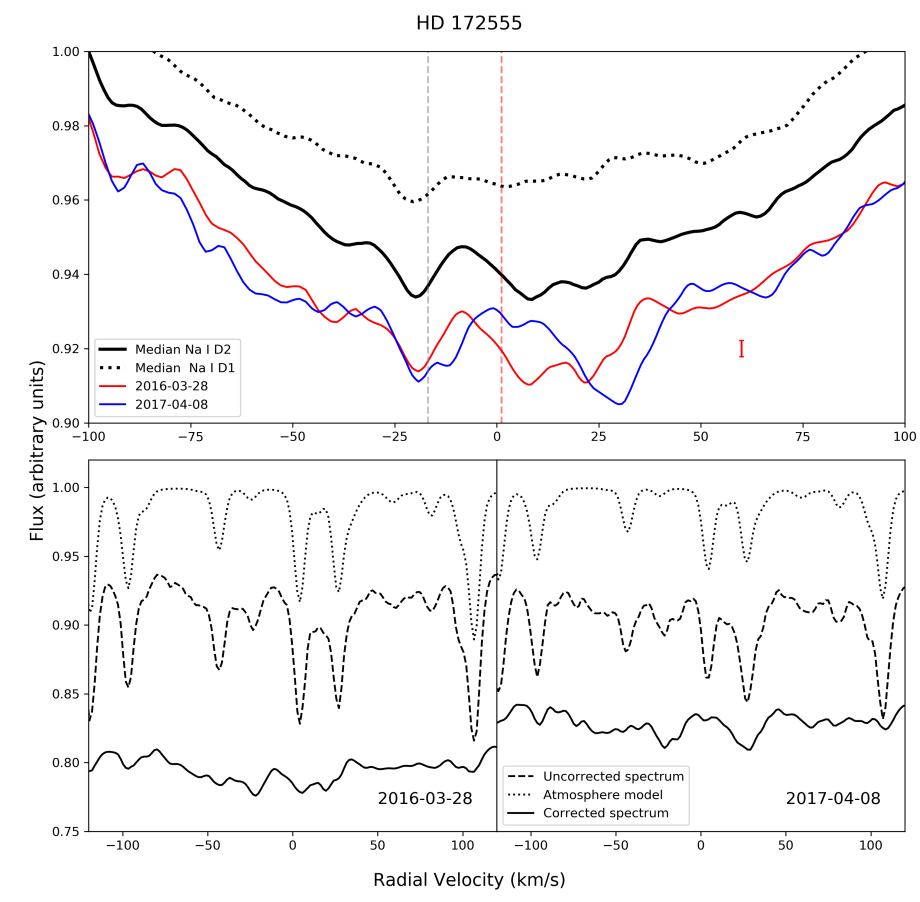

Fig. 23. Top panel: absorptions detected in the Na I D2 line of HD 172555. The black lines correspond to the median of all spectra for $\mathrm{Na}$ I D2 (solid line) and D1 (dotted line). The blue and red lines correspond to two different dates, where the variations in the $\sim 15 \mathrm{~km} \mathrm{~s}^{-1}$ $\mathrm{Na}$ I D2 component can be perceived. Bottom panel: examples of the telluric subtraction are plotted in order to show that this process is not the source of the variability. In both cases, spectra were obtained using FEROS. The red and grey vertical lines mark the stellar and ISM radial velocities, respectively.

feature varies its depth $(\sim 6.0 \sigma)$ when analysing the median spectra of different epochs (Fig. 24); thus, it most likely has a CS origin, at least in part. During the July 2016 observing run at Mercator, a very weak Ca II K blue-shifted absorption with an EW of $1.3 \mathrm{~m} \AA$ was apparent at a velocity of $\sim-35 \mathrm{~km} \mathrm{~s}^{-1}$; in addition, the spectrum from July 14, 2016 shows a Ca II K redshifted event at $\sim 25 \mathrm{~km} \mathrm{~s}^{-1}$ and EW $1.6 \mathrm{m \AA}$. This absorption was not detected in the $\mathrm{Ca}$ II $\mathrm{H}$ line. Since its value is consistent with a $3.1 \sigma$ detection, we consider the detection to be tentative. We note that Montgomery \& Welsh (2012) also noticed the variability of the narrow absorption as well as the presence of a FEB-like event with a velocity range $15-60 \mathrm{~km} \mathrm{~s}^{-1}$ in one of their spectra.

- HD 217782 (2 And) has three Ca II absorptions at velocities $\sim-17.1 \mathrm{~km} \mathrm{~s}^{-1},-9.2 \mathrm{~km} \mathrm{~s}^{-1}$, and $5.2 \mathrm{~km} \mathrm{~s}^{-1}$ (see Fig. A.1). Two of them, which are blue-shifted with respect to the radial velocity of the star, were also detected in $\mathrm{Na}$ I. The weakest feature at $5.1 \mathrm{~km} \mathrm{~s}^{-1}$ is at the stellar $v_{\text {rad }}$ and the velocity vector of the Hyades ISM cloud (Table B.3). While the stronger and narrower $-9.3 \mathrm{~km} \mathrm{~s}^{-1} \mathrm{Ca}$ II $\mathrm{K}$ feature remains unchanged (variations below $1 \sigma$ ), the -16.5 and likely the $5.1 \mathrm{~km} \mathrm{~s}^{-1}$ components present some variability (Fig. 25). Particularly, the $-16.5 \mathrm{~km} \mathrm{~s}^{-1}$ feature seems to experience some dynamical evolution and changes its depth and velocity within hours, for example, up to a $3 \sigma$ EW variation during the night of September 6-7, 2015 as well as a shape and depth change (Fig. 25, bottom left panel). Similar changes are also seen on other nights. Those changes, although much less remarkable, can tentatively be present in the weaker Ca II H feature, as seen in Fig. 26 where both $\mathrm{Ca}$ II non-photospheric lines of the mentioned night 
HD 182919

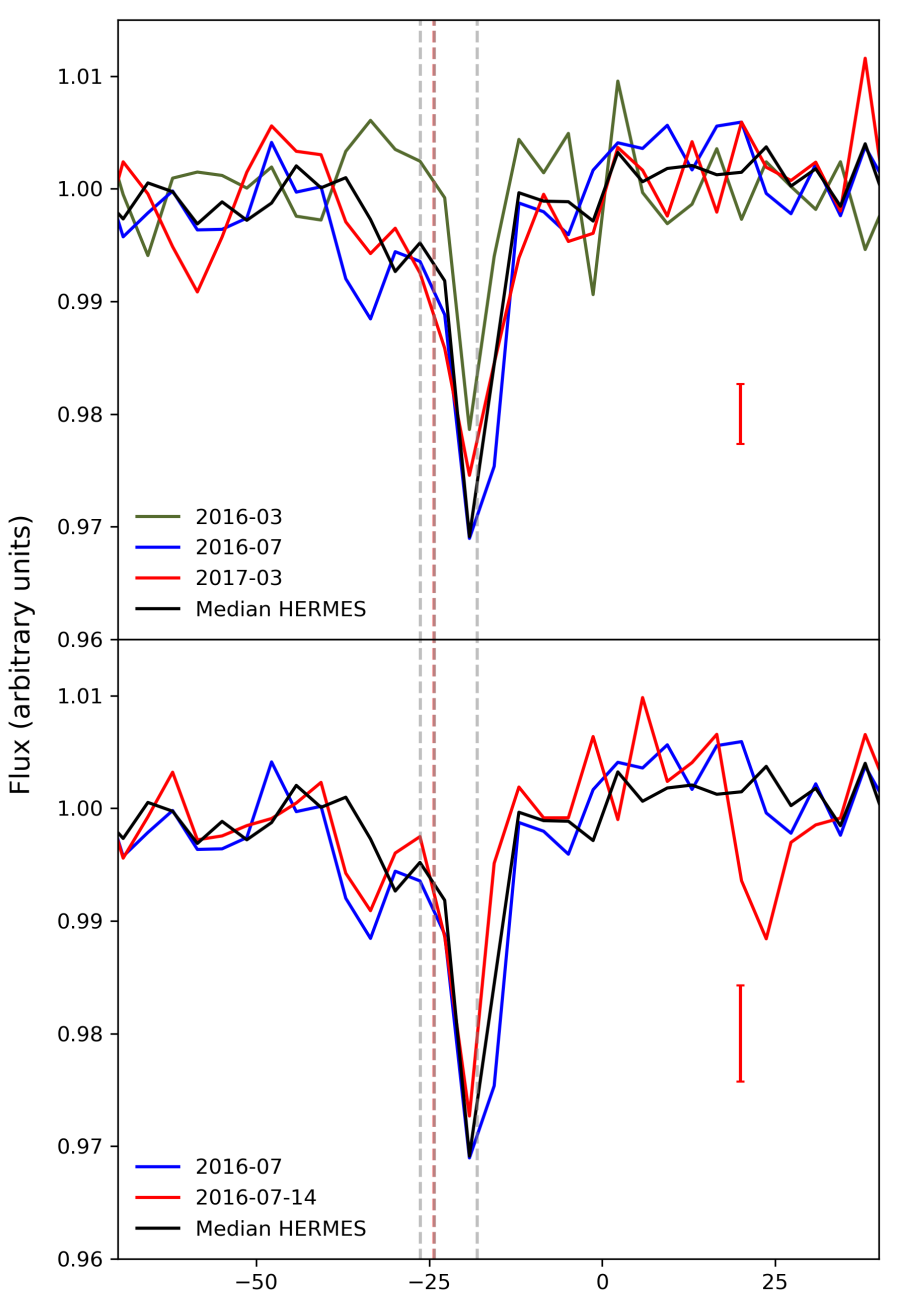

Radial Velocity $(\mathrm{km} / \mathrm{s})$

Fig. 24. Ca II K spectra of HD 182919 (5 Vul) taken with HERMES on the dates indicated in the labels. Top panel: variation of the features at $\sim-18$ and $\sim-35 \mathrm{~km} \mathrm{~s}^{-1}$. A possible FEB-like event at $\sim 25 \mathrm{~km} \mathrm{~s}^{-1}$ is visible in the bottom panel. The red and grey lines correspond to the radial velocity of the star and the velocity vector of Colorado clouds, respectively.

are shown. These results suggest the presence of CS gas around 2 And. Additionally, Cheng \& Neff (2003), Montgomery \& Welsh (2012), Welty et al. (1996) found similar UV and optical results.

\subsection{Summary of CS gas detections}

Figure A.1 shows the observed median Ca II K\&H and Na I D lines and the non-photospheric residuals, once the stellar contribution was subtracted, of the 60 stars that have narrow stable absorptions; radial velocities of the stars and the velocity vector of the ISM Colorado clouds velocities are also plotted. Table B.3 gives radial velocities, FWHM, equivalent widths, and column densities of the narrow features. Table 2 lists the stars showing variable absorptions detected in this work and also the stars reported in the literature to host sporadic events, but which were not detected by us.

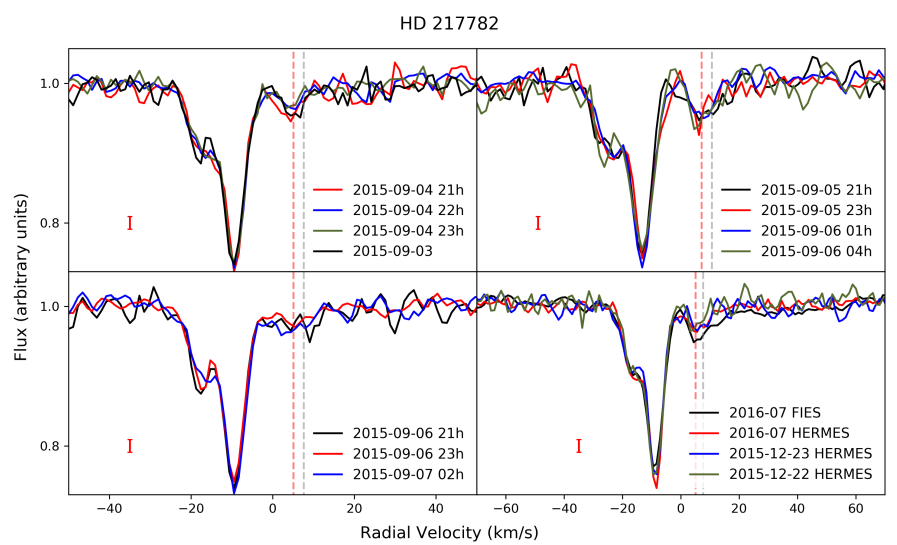

Fig. 25. Ca II K spectra of HD 217782 as indicated in the labels. Bottom right panel: the labels indicate the instrument used in each case. The rest of the spectra were obtained with HERMES. The dashed red and grey lines correspond to the radial velocity of the star and the velocity vector of Colorado clouds, respectively.

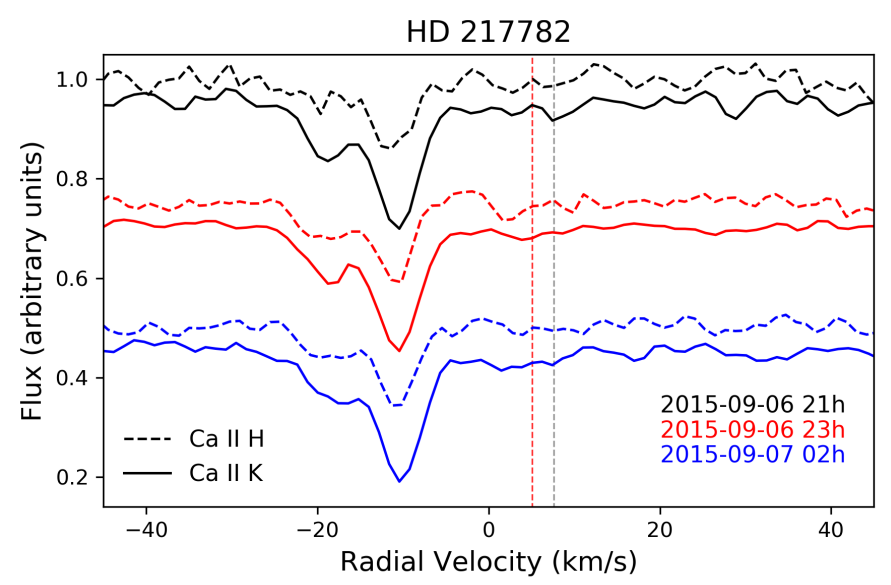

Fig. 26. Ca II H and K non-photospheric features of HD 217782 were taken on the night indicated in the labels. All spectra were taken using HERMES. A shift of 0.2 was introduced in the Y-axis to help differentiate the variations. The dashed red and grey lines correspond to the radial velocity of the star and the velocity vector of Colorado clouds, respectively.

We find evidence of hot CS gas in 32 objects out of the initial sample of 117 stars; 30 are in the form of stable nonphotospheric components and variable absorptions in the other two cases. Variable red- and/or blue-shifted events with respect to the radial velocity of the stars have been detected in 18 objects, including the serendipitous detection of HD 132200, which was not in the initial sample (Rebollido et al. 2018). Among those 18 objects, all but HD 110411 and HD 183324 have stable narrow features. These figures mean that we have found evidence of a close-in gaseous CS environment in $\sim 27 \%$ of the sample. We note, however, that it is not statistically significant as the selection criteria were highly biased and include, for instance, stars for which the presence of hot or cold CS gas was already known. Nonetheless, the figure does indicate that a non-negligible number of stars, particularly A-type (see below), are surrounded by CS gas, which can be detected by means of high resolution optical spectroscopy. We note that we are not considering the eight stars where the detected narrow feature has a dubious origin as we are unable to soundly attribute it either to a CS and/or ISM environment. 
The detected CS gas clearly has distinct origins. Red- and blue-shifted events are plausibly linked to the presence of FEBs, as in the well known case of $\beta$ Pic. Even in some cases, our spectra likely trace the dynamical evolution of those exocomets, that is, a change in depth and velocity. In none of the cases is the exocomet activity as rich as in $\beta$ Pic, which remains unique in this context. Stable absorption features in some stars are also likely related to exocomets and/or evaporation of grains in the immediate CS environment. In the case of shell stars, the nonphotospheric stable features, including many metallic shell lines of species such as Fe II or Ti II, are related to the shell itself, and they likely arise from mass loss phenomena experienced by the central star. Nonetheless, some shell stars also present sporadic red- or blue-shifted absorption events in Ca II reminiscent of exocomets. HD 37306 represents an extreme case, where we detected the appearance and disappearance of a strong shell spectrum but no trace of any exocomet-like event.

\section{Discussion}

While our stellar sample is heterogeneous and highly biased, we can still try to find some trends among the incidence of CS gas and some general properties of the stars and the different groups of stars according to the selection criteria. Figure 27 shows the HR diagram of the sample where the absolute magnitude $M_{V}$ and colour index $B-V$ magnitudes and parallaxes given in SIMBAD; the MS track was taken from Pecaut \& Mamajek (2013). To estimate $M_{V}$ we did not take the potential extinction towards the stars into account; nonetheless, the true loci of the individual stars in the HR diagram would not significantly alter the conclusions. Objects with evidence of hot CS gas (variable or stable) are A-type stars, and they are therefore located in the upper-left region of the diagram. This is in line with previous work; although, as far as we know they have only been concentrated in the study of A-type stars (e.g. Holweger et al. 1999; Welsh \& Montgomery 2018, and references therein). We only know of one later spectral type star, HD 109085 ( $~ \eta$ Crv, F2 V), for which one exocometary-like event has recently been reported, although it requires confirmation (Welsh \& Montgomery 2019); while cold CO is most likely present in this system (Marino et al. 2017), we did not find any traces of CS gas associated with this star (Rebollido et al. 2018, this work). In this respect, photometric transits are more efficient than spectroscopy in detecting exocomets around later type stars (e.g. Rappaport et al. 2018; Ansdell et al. 2019). The inability, at least up to now, of spectroscopy to detect exocomet signatures in late-type stars might be due to the concurrence of several causes, for example, stellar activity, which makes it extremely difficult to detect faint variable events superimposed on the profiles of the chromospheric active $\mathrm{Ca}$ II and Na I lines; also, late type stars usually have small rotational velocities so that narrow stable absorptions are practically indiscernible from the core of the narrow stellar lines.

It is obvious from Fig. 27 that stars with both stable and/or sporadic CS features tend to be in many cases located above the main sequence in the $\delta$ Scuti instability strip of the HR diagram (Breger 1979; Rodriguez et al. 1994). A few of the hotgas-bearing stars in our sample are identified as $\delta$ Scuti stars in SIMBAD, including HD 110411, HD 183324, and HD 192518; recently, Mellon et al. (2019) found that HD 156623 is also a $\delta$ Scuti star; we also point out that $\beta$ Pic itself has $\delta$ Scuti pulsations (Koen 2003; Mékarnia et al. 2017). In addition, the CS gas stars have a distinctly larger $v \sin i$. As expected (e.g. Nielsen et al. 2013), the highest $v \sin i$ values are found for the earlier

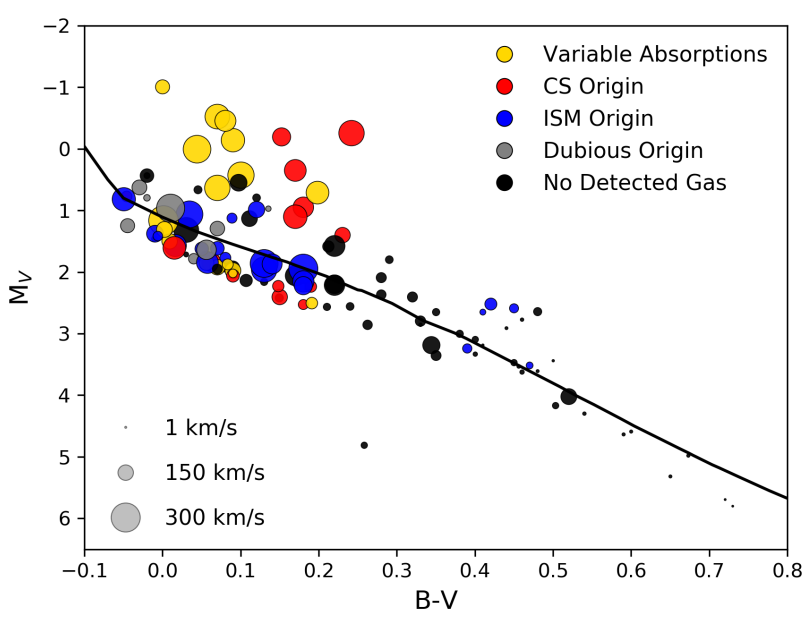

Fig. 27. Colour-Magnitude diagram of the whole sample. Colours represent stars with different non-photospheric features, while the size of the symbols is proportional to $v \sin i$.

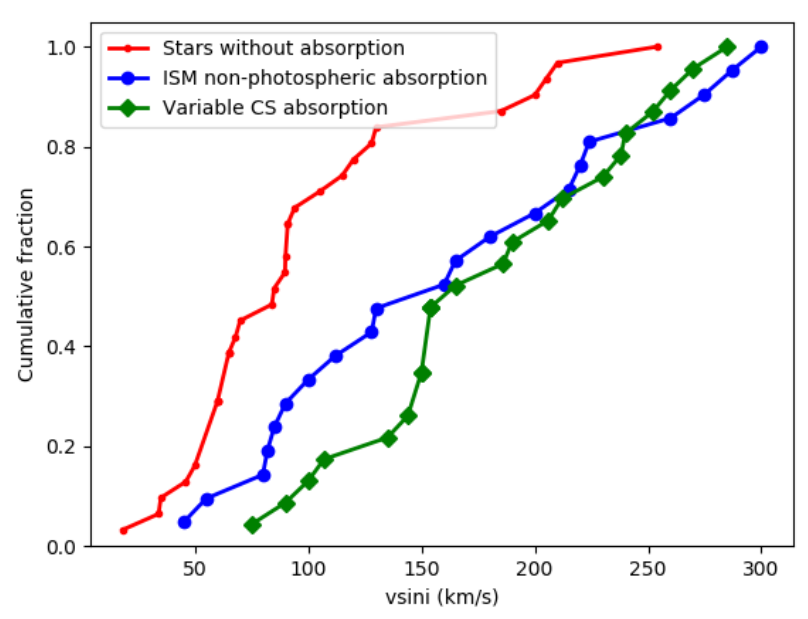

Fig. 28. Cumulative distribution functions of the sub-samples labelled in the plot.

spectral types (symbol sizes in Fig. 27 are proportional to $v \sin i$ ). Excluding stars later than F2, Fig. 28 shows the cumulative distribution functions (CDF) of the projected rotational velocity of early type stars without non-photospheric features, stars with features identified as ISM absorptions, and stars with variable events. It is evident from Fig. 28 that stars with non-photospheric ISM features or with variable CS events have larger $v \sin i$ values than stars without non-photosperic features. Table 3 shows the results of a Kolmogorov-Smirnov test separating those three sub-samples. We refer to Maldonado et al. (2012) for the meaning of the parameters $\mathrm{D}, p$-value, and $n_{\mathrm{eff}}$ in that table. In particular, the $p$-value indicates that the sub-sample of stars with ISM absorptions differ with a probability of $\sim 97 \%$ for the stars without non-photospheric absorptions; for the case of stars with variable events, the probability is nearly $100 \%$. At the same time, the results of the Kolmogorov-Smirnov test indicates that the sub-samples of ISM and variable events do not differ significantly. Nonetheless, a visual inspection of Fig. 28 suggests that there might be a paucity of the variable hot-gas-bearing stars with $v \sin i$ up to $\sim 150 \mathrm{~km} \mathrm{~s}^{-1}$ with respect to the sample of stars with ISM absorptions, which is lost in the statistical test when comparing the whole range of $v \sin i$. 
Table 3. Kolmogorov-Smirnov test comparing the projected rotational velocity distribution of the sub-sample of stars earlier than F2 without non-photospheric features, stars with absorptions with an ISM origin, and stars with variable events.

\begin{tabular}{lllll}
\hline \hline Sample 1 & Sample 2 & $\mathrm{D}$ & $p$-value & $n_{\mathrm{eff}}$ \\
\hline ISM absorp. & No absorp. & 0.39 & 0.030 & 12.52 \\
CS Var. absorp. & No absorp. & 0.66 & $6.376 \mathrm{e}-06$ & 12.20 \\
ISM absorp. & CS Var. absorp. & 0.30 & 0.222 & 10.98 \\
\hline
\end{tabular}

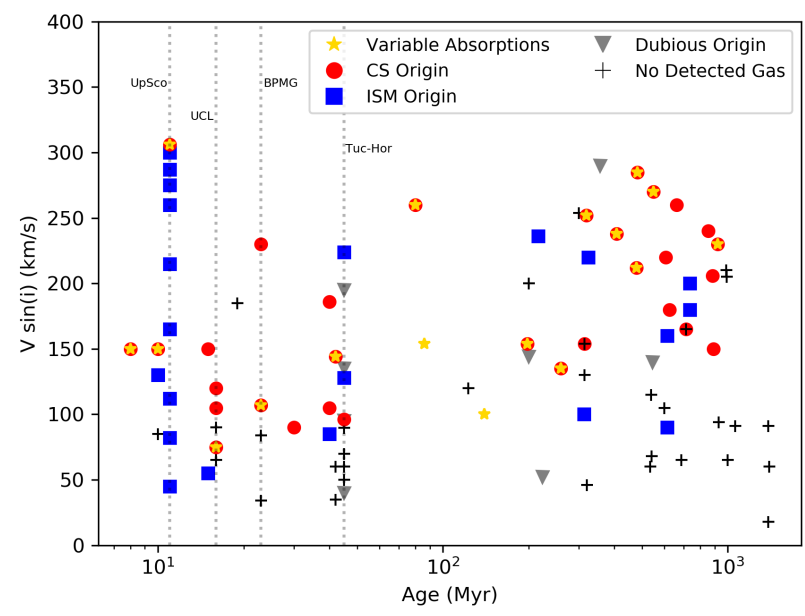

Fig. 29. Age versus $v \sin i$ of the stars in the sample. The symbols mark the type of absorptions detected for each object. Vertical dotted lines mark the location of some of the young associations: Upper Scorpious, Upper Centaurus Lupus, Beta Pic Moving Group, and Tucana-Horologium.

When considering the age of all stars with non-photospheric CS and ISM absorptions, $258 \%$ have ages below $100 \mathrm{Myr}$ (Table B.1). If we just consider stars plausibly hosting CS features, that figure is $\sim 51 \%$, and it reduces to $\sim 42 \%$ (11 out of the 26 stars) when the stars with variable features are considered. Thus, although ages of field stars might be highly uncertain and the nature of the variability of the features is not always due to exocomet-like events, which is the case of HD 37306, for example, stars with FEB-like events do not tend to be young objects, but they are distributed in a wide range of ages, from $\sim 10 \mathrm{Myr}$ to $\sim 1$ Gyr. All of this is clearly recognised in Fig. 29 where a plot of the rotational velocity of the stars (up to F2) versus age is shown. Stars with non-photospheric features are all younger than $1000 \mathrm{Myr}$, and their distribution is clearly modulated by stars in young clusters. This is mainly the case for UpSco, UCL, BPMG, and Tuc-Hor. Stars with CS features are found among the whole range of ages, and they have higher rotational velocities than stars without non-photospheric features. They also appear to have higher rotational velocities than stars with ISM features, thus reflecting the results from the Kolgomorov-Smirnov test above and the mentioned paucity of CS-feature stars with low $v \sin i$.

Out of the 32 stars in our sample with non-photospheric absorptions, we find, in many cases, coincident radial velocities (within the errors reported in Sect. 4.1) between the features observed in $\mathrm{Ca}$ and in $\mathrm{Na}$. Figure 30 shows the ratio of $\mathrm{Ca}$ II $\mathrm{K}$ and $\mathrm{Na}$ I D2 column densities against the $\mathrm{Ca}$ II $\mathrm{K}$ column density, which is colour coded for the attributed origin. In those cases where only one of the lines was detected, an upper limit was calculated using the EW uncertainty to compute the column

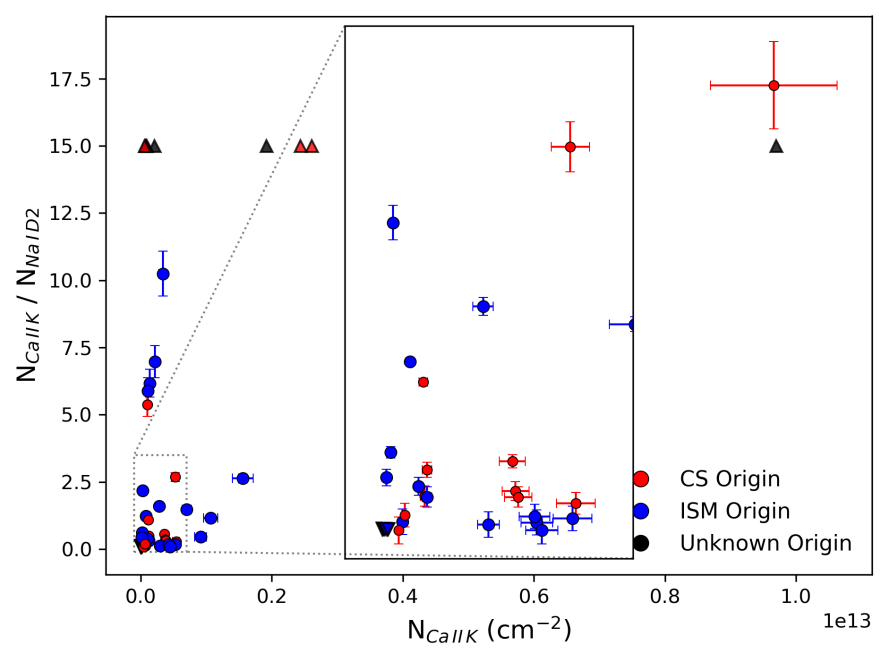

Fig. 30. Column densities of $\mathrm{Ca}$ II $\mathrm{K}$ and ratio of column density of $\mathrm{Ca}$ II K and Na I D2 of those absorptions of similar radial velocities. For the case where an absorption was detected in only one of the lines, triangles pointing up are upper limits and triangles pointing down are lower limits. The outlier in the upper-left corner of the plot is HD 42111. Colour denotes the suggested origin as in the legend.

density. The distribution of the stars in this diagram is consistent with other works (e.g. Welsh et al. 2010; Gudennavar et al. 2012). There is no clear separation between CS or ISM absorptions, that is, the ratios of their column densities do not show any significant trend when comparing them regarding their origin, which is in agreement with previous results (see Sect. 4.1.1, first paragraph). It is worth noticing though, that it seems that the presence of both $\mathrm{Ca}$ and $\mathrm{Na}$ components is more common in ISM absorptions. HD 42111 seems to behave as an extreme outlier in our sample as it has a large $N_{\text {Caii K }}$. However, this shell star has two, nearly blended, narrow absorptions (not discernible in the median spectrum), which might explain the large $N_{\text {Caii K }}$.

While objects in the sample are distributed in the sky without preferential locations (Fig. 1), we find a possible trend when examining distances. Stars without narrow absorptions (either CS or ISM) are located at $<50 \mathrm{pc}$, but there is no clear differentiation between stars with CS and ISM narrow absorptions. This could be due to the lower frequency of interstellar clouds at shorter distances, and the exponential growth of the number of stars with distance.

\subsection{Stars with debris discs}

To our knowledge, 76 out of the 117 stars in the sample are associated with a known debris disc (Table B.1). We find that 35 out of those 76 debris discs have at least a non-photospheric narrow feature (Tables B.3 and 4), and 15 among these 35 debris disc stars plausibly have at least one CS component; this figure does not include stars with an ambiguous origin as to the detected non-photospheric absorptions. Stable CS features at the velocity of the star have been interpreted as proof of a CS gas disc, and its persistence requires the presence of a braking mechanism that prevents the hot gas from being blown away by the strong radiation pressure from the star (e.g. Lagrange et al. 1998). Fernández et al. (2006) suggested that in $\beta$ Pic, such a mechanism could be exerted by the observed enhanced carbon abundance (see also Roberge et al. 2006; Brandeker 2011); a fact that has also been suggested for HD 9672 (Roberge et al. 2014). Among the stars in our sample with stable features, in addition to HD 9672 and 
Table 4. Debris disc stars in the sample with narrow non-photospheric features.

\begin{tabular}{lcc}
\hline \hline HD 3003 & HD 118232 & HD 146897 \\
HD 5267 & HD 125162 & HD 147137 \\
HD 9672 & HD 131488 & HD 156623 \\
HD 21620 & HD 131835 & HD 158352 \\
HD 32297 & HD 138813 & HD 172555 \\
HD 36546 & HD 142315 & HD 181296 \\
HD 37306 & HD 144587 & HD 182919 \\
HD 71043 & HD 144981 & HD 183324 \\
HD 71722 & HD 145554 & HD 188228 \\
HD 105850 & HD 145631 & HD 198160 \\
HD 109573 & HD 145689 & HD 221756 \\
HD 110058 & HD 145964 & \\
HD 110411 & HD 146606 & \\
\hline
\end{tabular}

Notes. Bold-faced stars have features of CS origin, and those in italics also have variable absorptions. HD 110411 and HD 183324 do not have narrow absorptions, but they have CS gas.

HD 36546 (see Sect. 4.2), that scenario might be at work for HD 32297 , where a carbon overabundance is suggested by the $3.7 \sigma$ Herschel detection of [C II] emission at $158 \mu \mathrm{m}$ (Donaldson et al. 2013).

Concerning variable absorptions, 12 out of the total 18 stars showing variability either in one or both of the Ca II H\&K lines or in one or both of the $\mathrm{Na} I \mathrm{D}$ lines also have excesses associated with the presence of a debris discs (Tables 2 and 4). All these stars are younger than $200 \mathrm{Myr}$.

In all cases, except for HD 37306, transient red- or blueshifted events attributed to exocomets have been observed. The observed variability in HD 37306 is due to the appearance, and later disappearance, of a strong shell spectrum (see above). Complementary to these results, the rest of the stars with variable features listed in Table 2, that is, 14 stars, do not host a debris disc, and they are older than $200 \mathrm{Myr}$. We point out, however, that in the four cases involving HD 39182, HD 64145, HD 132200, and HD 138629, there is no observational information concerning the potential presence of a debris disc, and in the two cases involving HD 132200 and HD 138629, we do not have information about their age.

In parallel, to our knowledge, 36 out of the 76 debris discs were spatially resolved after the compilations by N. Pawellek and A. Krivov ${ }^{4}$ and C. McCabe, I.H. Jansen, and K. Stapelfeldt ${ }^{5}$. Furthermore, 28 out of those 36 resolved debris disc stars have spectral types earlier than F2 and, therefore, they are sensitive enough to show the presence of exocometary signals. Also, Moór et al. (2017) report inclinations for the A1 stars HD 121617 and HD 131488. Table 5 lists the early-type 30 resolved debris discs together with their corresponding inclination angles, taken from the mentioned catalogues. Among the resolved discs, 17 have non-photospheric features (Table 5). In the following six cases, the absorption features are more plausibly interstellar or ambiguous: HD 71722, HD 125162, HD 131835, HD 138813, HD 146897, and HD 188228. In 11 stars, a CS origin seems to be the most reasonable one for at least one of the observed non-photospheric absorption features; eight out of these 11 stars have variable absorptions. However, based on a relatively low number of objects, inspection of the inclination

\footnotetext{
4 https://www.astro.uni-jena.de/index.php/theory/ catalog-of-resolved-debris-disks.html

5 https://www. circumstellardisks.org/
}

Table 5. Resolved debris discs and inclination angles.

\begin{tabular}{|c|c|c|c|c|c|}
\hline Star & $i^{\circ}$ & star & $i^{\circ}$ & star & $i^{\circ}$ \\
\hline HD 9672 & 79 & HD 71722 & 78 & $H D 131488^{(2)}$ & 82 \\
\hline HD 14055 & 83 & HD 74873 & 27 & HD 131835 & 75 \\
\hline HD 15115 & 80 & HD 95418 & 84 & HD 139006 & 80 \\
\hline HD 21997 & 26 & HD 102647 & 30 & HD 138813 & 28 \\
\hline HD 27290 & 69 & HD 109085 & 35 & HD 146897 & 84 \\
\hline HD 28355 & 76 & HD 109573 & 76 & $H D 156623$ & 30 \\
\hline HD $31295^{(*),(1)}$ & & HD $110058^{(\dagger)}$ & 50 & HD 172555 & 75 \\
\hline HD 32297 & 90 & HD 110411 & 70 & HD 181296 & 90 \\
\hline HD 36546 & 75 & HD $121617^{(2)}$ & 37 & HD 183324 & 2 \\
\hline HD 38206 & 60 & HD 125162 & 48 & HD 188228 & 49 \\
\hline
\end{tabular}

Notes. Narrow non-photospheric absorption features are in bold-faced while those in italics have at least one component attributed to a CS origin. HD 110411 and HD 183324 do not have narrow absorptions, but they have CS gas. ${ }^{(*)}$ Resolved debris disc with unreported inclination angle; ${ }^{(\dagger)}$ Kasper et al. (2015) report an edge-on inclination.

References. (1) Draper et al. (2016); (2) Moór et al. (2017).

angles in Table 5 reveals: i) there is not a preferential distinction between discs seen edge- or polar-on for those debris discs without non-photospheric absorptions; ii) a similar result is seen for those stars with ISM features; and iii) the trend is clearly different when we inspect debris discs with CS absorptions, that is, most of them are clearly seen at $i>70^{\circ}(\sim 72 \%)$, and this trend is reinforced when only those discs with variable features are considered. In other words, debris discs associated with stars hosting CS absorptions tend to be seen edge-on, while debris discs associated with stars without non-photospheric absorptions or stars with ISM features do not show a preferential inclination. This result is consistent with the fact that the detection of CS features, that is, hot gas, is favoured when the systems are seen close to edge-on as well as with the large projected rotational velocities shown by the stars with CS gas compared to those without such gas. We note that the observed trend, which is just a geometrical effect, does not exclude the existence of hot gas, that is, the eventual existence of comet-like bodies around the debris discs systems seen away from edge-on. A similar trend was already pointed out by Rebollido et al. (2018) in their analysis of debris discs with measurable amounts of cold gas seen in emission in the far-IR and (sub-)millimetre wavelength regimes.

\subsection{Near infra-red excesses}

There are 22 stars (Table B.1) in our sample that were explicitly taken from the literature when searching for hot excesses in the $\mathrm{H}(1.6 \mu \mathrm{m})$ and $\mathrm{K}(2.2 \mu \mathrm{m})$ bands (Table B.1). In addition, HD 172555 also presents such an excess (Absil et al. 2013; Ertel et al. 2014, 2016; Nuñez et al. 2017).

Within the stars in our sample with near-IR excesses that are plausibly due to hot dust (i.e. excluding stars where binarity is the cause of the excess), 11 stars have spectral types that are earlier than F2. Additionally, six out of those 11 stars present nonphotospheric features, which were either detected in this work or in previous ones (Table 6).

To assess the significance of the incidence of CS absorption around hot dust stars, we consider all of the reported, 31 near-IR excess stars (Absil et al. 2013; Ertel et al. 2014; Nuñez et al. 2017). Excluding the binaries, 14 stars, including the 11 studied by us plus Vega, $\beta$ Pic, and HD 210049 ( $\mu$ PsA), have 
Table 6. Stars showing near-IR excesses.

\begin{tabular}{lcr}
\hline \hline Name & $\begin{array}{c}\text { Non-photospheric } \\
\text { absorption }\end{array}$ & H/K excess \\
\hline HD 2262 & Yes ${ }^{(*)}(1)$ & $(2)$ \\
HD 28355 & No & $(2)$ \\
HD 40136 & No & $(1)$ \\
HD 56537 & Yes $^{(2)}$ & $(1)$ \\
HD 102647 & No & $(1)$ \\
HD 108767 & Yes $^{(2,3)}$ & $(2)$ \\
HD 172555 & Yes $^{(3,4)}$ & $(2)$ \\
HD 177724 & Yes $^{(3)}$ & $(1,3)$ \\
HD 187642 & No & $(1,3)$ \\
HD 203280 & No & $(1)$ \\
HD 210418 & Yes $^{(3)}$ & $(3)$ \\
\hline
\end{tabular}

Notes. ${ }^{(*)}$ Not clear if exocomet-like or stellar mass loss events.

References. References for non-photospheric absorptions: (1): Welsh \& Montgomery (2018); (2): Welsh \& Montgomery (2015); (3) this work; (4) Kiefer et al. (2014b). References for $\mathrm{H} / \mathrm{K}$ excess: (1): Absil et al. (2013), (2): Ertel et al. (2014), (3) Nuñez et al. (2017).

Table 7. Shell stars.

\begin{tabular}{lll}
\hline \hline Star & Star & Star \\
\hline HD 256 & HD 50241 & HD 148283 \\
HD 21688 & HD 77190 & HD 158352 \\
HD 37306 & HD 85905 & HD 168646 \\
HD 39182 & HD 98058 & HD 192518 \\
HD 39283 & HD 118232 & HD 196724 \\
HD 42111 & HD 138629 & HD 217782 \\
\hline
\end{tabular}

Notes. Stars with non-photospheric CS features are bold-faced while those with variability are emphasised.

spectral types earlier than F2. Among those 14 stars, five have variable absorption features (HD 2262, HD 56573, HD 108767 , HD 172555, and $\beta$ Pic); two stars have an ambiguous CS and ISM narrow feature (HD 177724, and HD 210418); two stars are associated with pole-on debris discs (HD 102647 and Vega), that is, an unfavourable orientation when trying to detect CS features; one star (HD 28355) has a resolved debris disc with an inclination angle of $i=70^{\circ}$, but our spectra do no reveal any nonphotospheric absorption; and for the last four stars (HD 40136, HD 187642, HD 203280, and HD 210049), the orientation of the system is unknown. If we assume random orientations, the probability of observing a system with an inclination higher than $65^{\circ}-70^{\circ}$, that is, edge-on or close to it, lies between $\sim 42 \%$ and $34 \%$, respectively, which is similar to the percentage of stars with hot CS gas among the hot dust stars. We also note that it is in agreement with the percentage of edge-on discs, either debris or other types of discs, which can be found in the Catalogue of Resolved Discs compiled by C. McCabe, I.H. Jansen, and K. Stapelfeldt. Given those figures, a relationship between hot dust and hot CS gas is suggested, which should be confirmed by increasing the sample of stars with the appropriate near-IR interferometric as well as UV and optical spectroscopic data.

\subsection{Ti II: shell stars}

In addition to the stars selected on the basis of their Ti II lines, there are a number of stars which have been classified as shell stars (Abt 2008; Lagrange-Henri et al. 1990b; Hauck \& Jaschek
Table 8. EW of the Mg II $4481 \AA$ and O I $7744 \AA$ lines of previously known $\lambda$ Boo stars, together with stars of the sample with similar characteristics.

\begin{tabular}{lrrc}
\hline \hline Star & $\begin{array}{r}\text { EW Mg II } \\
(\AA)\end{array}$ & $\begin{array}{r}\text { EW O I } \\
(\AA)\end{array}$ & $\lambda$ Böo \\
\hline HD 31295 & 0.188 & 0.692 & Yes \\
HD 32297 & 0.336 & 0.767 & New \\
HD 36546 & 0.231 & 0.748 & New \\
HD 39182 & 0.413 & 1.010 & Shell \\
HD 39283 & 0.347 & 0.720 & Yes \\
HD 42111 & 0.424 & 1.258 & Shell \\
HD 71722 & 0.294 & 0.802 & New \\
HD 74873 & 0.192 & 0.711 & Yes \\
HD 110058 & 0.310 & 0.774 & New \\
HD 110411 & 0.213 & 0.689 & Yes \\
HD 125162 & 0.087 & 0.717 & Yes \\
HD 145964 & 0.388 & 0.532 & Yes \\
HD 156623 & 0.366 & 0.802 & New \\
HD 168646 & 0.418 & 0.937 & Shell \\
HD 177724 & 0.294 & 0.726 & New \\
HD 183324 & 0.114 & 0.738 & Yes \\
HD 198160 & 0.362 & 0.745 & Yes \\
HD 198161 & 0.378 & 0.762 & Yes \\
HD 210418 & 0.385 & 0.836 & Yes \\
HD 217782 & 0.435 & 0.877 & Yes \\
HD 221756 & 0.289 & 0.778 & Yes \\
\hline
\end{tabular}

Notes. The new $\lambda$ Boo candidates are indicated.

2000; Roberge \& Weinberger 2008). Table 7 lists, according to our knowledge, the shell stars in our sample. No Ca II or Na I non-photospheric absorptions were observed towards HD 39283 and HD 77190; in the cases of HD 118232 and HD 196724, the origin of the Ca II absorption is likely ISM or ambiguous. As a matter of fact, the spectra of these four stars do not reveal any prominent shell-like characteristics. The rest of the stars show triangular-like CS absorptions (bold-faced in the table); those stars with variable features are also emphasised. Not all absorptions can be attributed to FEB-like events; for example, the stable absorption seen towards HD 192518 is clearly formed in a gaseous shell, while the variability in HD 37306 is due to the appearance and disappearance of a shell around this star, as previously mentioned. Nonetheless, 11 stars do present variable events, most of them are attributable to exocomets even though our spectra might be tracing their dynamical evolution of some events by changing their depths and velocities.

\section{4. $\lambda$ Boo stars}

The sample contains 12 objects previously classified as $\lambda$ Boo type stars (Table 8), although three of them, HD 39283, HD 210418, and HD 217782, have recently been classified as nonmembers of this stellar class (Murphy et al. 2015, and references therein). Several criteria have been used to classify $\lambda$ Boo stars (e.g. Murphy et al. 2015; Gray et al. 2017), including optical line ratios between volatiles, such as CNO, and heavier elements (e.g. $\mathrm{Mg}$ ), which are useful when ascribing stars to this stellar class (Cheng et al. 2017). This criterion is based on the basic definition of the $\lambda$ Boo stars, that is, stars with a remarkably low abundance of heavy (e.g. Fe, $\mathrm{Al}, \mathrm{Mg}$ ) elements, while volatiles, such as $\mathrm{CNO}$, have near solar abundances (e.g. Baschek \& Slettebak 1988). In order to eventually find new $\lambda$ 


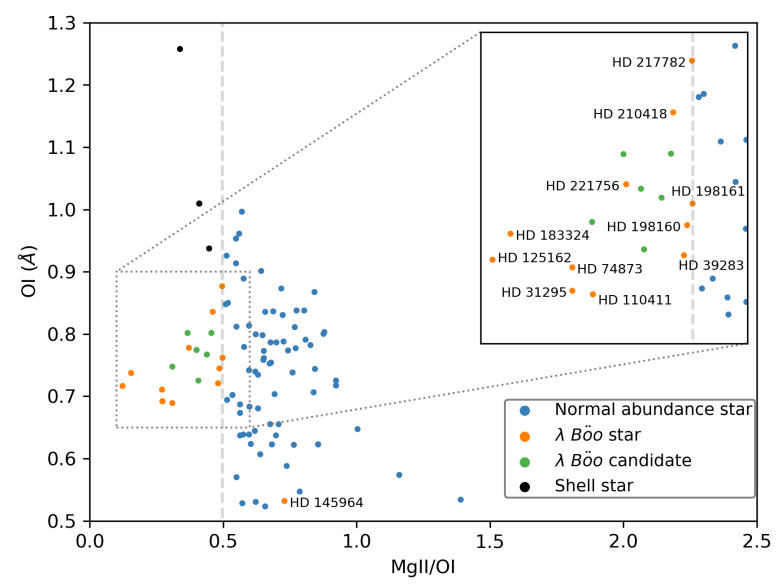

Fig. 31. Ratio O I (7744 $\AA$ ) and Mg II (4481 $⿱$ ) versus the EW of O I. The orange dots mark stars which were previously classified as $\lambda$ Boo stars; the green dots mark new $\lambda$ Boo candidates; and the blue dots mark the stars with normal abundances. The vertical dashed line delimits the locus of bona fide $\lambda$ Boo stars (see text).

Boo candidates, we measured, in all stars earlier than F2 in our sample, the ratio of the Mg II $4481 \AA$ and the O I $7774 \AA$ triplet, which are strong and easy to measure in our spectra.

Figure 31 is a plot of the $\mathrm{EW}$ of the $\mathrm{O} \mathrm{I}$ line versus the ratio of $\mathrm{Mg}$ II to $\mathrm{O}$ I lines. All identified $\lambda$ Boo stars in the sample are clearly located on the left in that plot and are well separated from the bulk of the stars. The exception is HD 145964, which is classified as a weak $\lambda$ Boo star, by Welsh \& Montgomery (2013) based on the measurements of Abt $\&$ Morrell (1995). The identified $\lambda$ Boo stars have low metallicities $([\mathrm{Fe} / \mathrm{H}]<-0.25)$, excluding $\mathrm{HD}$ 145964 and HD 217782. A vertical dashed line at MgII /O I $<0.49$ marks the limit for the identified $\lambda$ Boo objects in our sample. This figure is the one for the $\lambda$ Boo stars HD 198160 and HD 198161, and approximately the one for those removed from the class by Murphy et al. (2015) stars. We note that six stars are additionally located at similar locations in Fig. 31 as the $\lambda$ Boo stars, and they also have low metallicities (Table B.2). We consider that those stars are new candidates (Table 8). In addition, three shell stars, HD 39182, HD 42111, and HD 168646, have a low Mg II/O I ratio but a very high EW (O I) and metallicities $>0.0$.

With respect to the presence of non-photospheric narrow and/or variable features, 15 out of the $18 \lambda$ Boo stars (including the new candidates) listed in Table 8 present evidence of non-photospheric gas in their spectrum: In five cases, the feature is interstellar; in two cases, the origin is ambiguous; and the remaining eight stars (44\% of the $\lambda$ Boo stars) have exocometary-like events. Comparing this figure to the $26 \%$ incidence of exocometary-like events of the whole sample, there seems to be an enhanced probability for $\lambda$ Boo stars to have such events. A connection between metal-poor stars and the presence of debris discs or remainings of planet formation processes has been suggested before in the literature (Jura 2015; Murphy \& Paunzen 2017), as heavier elements are blown away by radiation pressure, while volatile elements are accreted onto the star (see Draper et al. 2016, and references therein). Therefore, exocomets represent a rather likely scenario that could replenish the atmosphere of $\lambda$ Boo stars of $\mathrm{C}, \mathrm{N}, \mathrm{O}$, and $\mathrm{S}$ elements.

\section{Conclusions}

We have presented the observational results of a systematic study aiming to detect and monitor hot gas attributed to the presence of exocomets in the CS environment. The study is based on the analysis of the $\mathrm{Ca}$ II $\mathrm{K} \& \mathrm{H}$ and $\mathrm{Na}$ I D lines in a heterogeneous and biased sample of 117 main-sequence late $B$ to $G$ type stars. This is the largest systematic study searching for exocomets that has been carried out so far. The main results are as follows.

Narrow non-photospheric ISM or CS absorptions have been detected towards $\sim 50 \%$ of the sample (60 stars). Among the stars with those absorptions, at least one of the detected narrow features can be attributed to CS gas in 30 objects, that is, $26 \%$ of the whole sample. This figure is not statistically significant as the studied sample includes stars with previously detected CS gas, but it does show that gas in the CS environment of A-type stars is relatively common. In some stars, the gas originated from a CS shell surrounding the stars; in some other cases, the narrow absorptions can be attributed to the evaporation of exocomets or to gas released by dust grain collisions or evaporation.

Sporadic red-shifted and blue-shifted events that, in some cases (but not all), might be due to $\beta$ Pic-like exocomets have been found in the CS environment of 16 stars; six of which are new to the literature. This includes HD 132200, which is not contained in the original sample. In a few cases, our spectra seem to trace the dynamical evolution of such events as suggested by changes in their velocity and depth. Nonetheless, the exocometary-like activity in our stars, maybe with the exception of $\phi$ Leo (see Eiroa et al. 2016), is very poor compared with $\beta$ Pic, which remains a unique object.

The variations observed towards the two other stars, namely HD 256 (HR 10) and HD 37306 (HR 1919), do not require the presence of exocomets for their interpretation. The variability of the CS features in HR 10 is mainly due to the binary character of this star. A detailed analysis has been carried out in a separate paper (Montesinos et al. 2019). In the particular case of HD 37306, our spectra have witnessed the appearance and disappearance of a strong shell around this star. There are no hints in our data of exocometary events in this star, although we point out that some shell stars in our sample do show such events.

Hot CS gas is only detected towards stars earlier than A9, whcih is in line with previous works. The F2 V star $\eta \mathrm{Crv}$ is the only star for which $\beta$ Pic-like events with a $2.9 \sigma$ detection have been claimed (Welsh \& Montgomery 2019). This paucity in detecting exocometary events around late type stars might be due to the inability of spectroscopy to detect faint non-photospheric absorptions on top of cool photospheres. In this respect, photometric transits have demonstrated that they are competitive and successful in detecting exocomets around late type stars.

Hot gas-bearing main sequence stars have distinctly higher projected rotational velocities and spread over a large range of ages, from $\sim 10 \mathrm{Myr}$ up to at least $\sim 1$ Gyr. Some of them are also known to present $\delta$ Scuti pulsations, as $\beta$ Pic itself.

Exocometary-like events are often associated with edge-on debris disc stars, in particular towards those with cold gas (see also Rebollido et al. 2018). This result is interpreted as a geometrical effect, but it does suggest that debris disc stars with non-photospheric absorptions (i.e. hot gas) are excellent targets to search for in the far-IR and (sub-)millimetre spectral range for the presence of cold gas released by the evaporation of solid bodies at distances relatively far from the central star. We note that not all stars with FEB-events are associated with a debris disc. It cannot be excluded that this is due to the current, limited sensitivity to detect debris discs, $L_{\text {dust }} / \mathrm{L}_{*} \sim 10^{-6}$.

We find that FEB-like events are detected towards $17 \%$ of stars with near-IR excesses, denoting the presence of hot exozodies. Both hot dust and gas might be related phenomena, 
although more observations are needed to confirm or deny the trend pointed out in our study.

Our sample includes $18 \lambda$ Bootis stars, with six new candidates found in this work. A relevant fraction of them, eight out the 18 stars, have FEB-like events, suggesting again that both phenomena could be related.

Acknowledgements. The authors wish to thank the careful reading of the manuscript and comments by the referee which have helped to improve the content of this work. Based on observations made with the Mercator Telescope, operated on the island of La Palma by the Flemmish Community, and the Nordic Optical Telescope, operated by the Nordic Optical Telescope Scientific Association, at the Spanish Observatorio del Roque de los Muchachos of the Instituto de Astrofísica de Canarias. Based on observations made with ESO Telescopes at the La Silla Observatory under programmes 099.A-9029(A),099.A-9004(A) and 094.A-9012. Also based on observations made with the TIGRE telescope funded and operated by the Universities of Hamburg, Guanajuato and Liège. We thank David Montes for providing the December 2018 Mercator spectra and to Santos Pedraz and Ana Guijarro for the CARMENES spectra of HD 37306. We thank Sam Kim and Markus Rabus for the FEROS spectra obtained in September 2017. I.R. thanks Angela Hempel for her support with FEROS observations in March 2016 and April 2017, and the Calar Alto Observatory staff, where a large part of this paper was written. Partially based on data obtained from the ESO Science Archive Facility, programs 096.C-0876, 076.B-0055, 185.D-0056, 082.B-0610 072.C-0488, 0100.C-0090, 093.C-0409, 081.D-0610, 080.A-9006, 184.C-0815, 077.C-0295. C.E., G.M., B.M., I.R., and E.V. are supported by Spanish gran AYA 2014-55840-P. H.C. acknowledges funding from the ESA Research Fellowship Programme. J. O., A. B., and D. I. acknowledge support from the ICM (Iniciativa Científica Milenio) via the Nucleo Milenio de Formación planetaria grant. J. O. acknowledges support from the Universidad de Valparaíso and from Fondecyt (grant 1180395). A. B. acknowledges support from Fondecyt (grant 1190748). A. M. acknowledges the support of the Hungarian National Research Development and Innovation Office NKFIH Grant KH-130526. This research has made use of the SIMBAD database, operated at CDS, Strasbourg, France. This work has used Seth Redfield's Colorado model of interstellar clouds an his online tool "LISM Kinematic Calculator". This work has also used the debris disc catalogues "Resolved debris discs" from Jena University compiled by N Pawellek and A. Krivov; and the one in circumstellardisks.org compiled and mantained by C. McCabe, I. H. Jansen and K. Stapelfeldt.

\section{References}

Absil, O., di Folco, E., Mérand, A., et al. 2006, A\&A, 452, 237

Absil, O., Defrère, D., Coudé du Foresto, V., et al. 2013, A\&A, 555, A104

Abt, H. A. 2008, ApJS, 174, 499

Abt, H. A., \& Morrell, N. I. 1995, ApJS, 99, 135

Abt, H. A., \& Moyd, K. I. 1973, ApJ, 182, 809

Abt, H. A., Tan, H., \& Zhou, H. 1997, ApJ, 487, 365

Ansdell, M., Gaidos, E., Jacobs, T. L., et al. 2019, MNRAS, 483, 3579

Armitage, P. J. 2010, Astrophysics of Planet Formation (Cambridge, UK: Cambridge University Press)

Ballering, N. P., Rieke, G. H., Su, K. Y. L., \& Montiel, E. 2013, ApJ, 775, 55

Baschek, B., \& Slettebak, A. 1988, A\&A, 207, 112

Bell, C. P. M., Mamajek, E. E., \& Naylor, T. 2015, MNRAS, 454, 593

Bertin, P., Lallement, R., Ferlet, R., \& Vidal-Madjar, A. 1993, A\&A, 278, 549

Beust, H., \& Morbidelli, A. 2000, Icarus, 143, 170

Beust, H., Vidal-Madjar, A., Ferlet, R., \& Lagrange-Henri, A. M. 1990, A\&A, 236, 202

Beust, H., Vidal-Madjar, A., \& Ferlet, R. 1991, A\&A, 247, 505

Beust, H., Lagrange, A.-M., Crawford, I. A., et al. 1998, A\&A, 338, 1015

Bochanski, J. J., Faherty, J. K., Gagné, J., et al. 2018, AJ, 155, 149

Bonsor, A., Raymond, S. N., Augereau, J.-C., \& Ormel, C. W. 2014, MNRAS, 441,2380

Boyajian, T. S., LaCourse, D. M., Rappaport, S. A., et al. 2016, MNRAS, 457 3988

Brandeker, A. 2011, ApJ, 729, 122

Brandeker, A., Liseau, R., Olofsson, G., \& Fridlund, M. 2004, A\&A, 413, 681

Breger, M. 1979, PASP, 91, 5

Cataldi, G., Moór, A., Ohashi, N., et al. 2019, Res. Notes Am. Astron. Soc., 3 39

Chen, C. H., \& Jura, M. 2003, ApJ, 582, 443

Chen, C. H., Patten, B. M., Werner, M. W., et al. 2005, ApJ, 634, 1372

Chen, C. H., Mittal, T., Kuchner, M., et al. 2014, ApJS, 211, 25

Cheng, K.-P., \& Neff, J. E. 2003, AJ, 125, 868

Cheng, K.-P., Neff, J. E., Johnson, D. M., et al. 2017, AJ, 153, 39

Cotten, T. H., \& Song, I. 2016, ApJS, 225, 15
Currie, T., Lisse, C. M., Sicilia-Aguilar, A., Rieke, G. H., \& Su, K. Y. L. 2011, ApJ, 734, 115

Currie, T., Guyon, O., Tamura, M., et al. 2017, ApJ, 836, L15

David, T. J., \& Hillenbrand, L. A. 2015, ApJ, 804, 146

Desidera, S., Covino, E., Messina, S., et al. 2015, A\&A, 573, A126

de Zeeuw, P. T., Hoogerwerf, R., de Bruijne, J. H. J., Brown, A. G. A., \& Blaauw, A. 1999, AJ, 117,354

Donaldson, J. K., Roberge, A., Chen, C. H., et al. 2012, ApJ, 753, 147

Donaldson, J. K., Lebreton, J., Roberge, A., Augereau, J.-C., \& Krivov, A. V. 2013, ApJ, 772, 17

Draper, Z. H., Matthews, B. C., Kennedy, G. M., et al. 2016, MNRAS, 456, 459

Duchêne, G., Arriaga, P., Wyatt, M., et al. 2014, ApJ, 784, 148

Eggleton, P. P., \& Tokovinin, A. A. 2008, MNRAS, 389, 869

Eiroa, C., Marshall, J. P., Mora, A., et al. 2013, A\&A, 555, A11

Eiroa, C., Rebollido, I., Montesinos, B., et al. 2016, A\&A, 594, L1

Ertel, S., Absil, O., Defrère, D., et al. 2014, A\&A, 570, A128

Ertel, S., Defrère, D., Absil, O., et al. 2016, A\&A, 595, A44

Faraggiana, R., \& Bonifacio, P. 1999, A\&A, 349, 521

Faramaz, V., Ertel, S., Booth, M., Cuadra, J., \& Simmonds, C. 2017, MNRAS, 465, 2352

Ferlet, R., Vidal-Madjar, A., \& Hobbs, L. M. 1987, A\&A, 185, 267

Fernández, R., Brandeker, A., \& Wu, Y. 2006, ApJ, 643, 509

Frandsen, S., \& Lindberg, B. 2000, The Third MONS Workshop: Science Preparation and Target Selection, eds. T. Teixeira, \& T. Bedding, 163

Gáspár, A., Rieke, G. H., \& Balog, Z. 2013, ApJ, 768, 25

Geiler, F., \& Krivov, A. V. 2017, MNRAS, 468, 959

Génova, R., \& Beckman, J. E. 2003, ApJS, 145, 355

Gontcharov, G. A. 2012, Astron. Lett., 38, 771

Grady, C. A., McCollum, B., Rawley, L. A., et al. 1996a, ApJ, 464, L183

Grady, C. A., Perez, M. R., Talavera, A., et al. 1996b, ApJ, 471, L49

Grady, C. A., Brown, A., Welsh, B., et al. 2018, AJ, 155, 242

Gray, R. O., \& Corbally, J. C. 2009, Stellar Spectral Classification

Gray, R. O., Riggs, Q. S., Koen, C., et al. 2017, AJ, 154, 31

Greaves, J. S., Holland, W. S., Matthews, B. C., et al. 2016, MNRAS, 461, 3910 Gudennavar, S. B., Bubbly, S. G., Preethi, K., \& Murthy, J. 2012, ApJS, 199, 8

Hauck, B., \& Jaschek, C. 2000, A\&A, 354, 157

Hempel, M., \& Schmitt, J. H. M. M. 2003, A\&A, 408, 971

Hobbs, L. M., Welty, D. E., Lagrange-Henri, A. M., Ferlet, R., \& Vidal-Madjar, A. 1988, ApJ, 334, L41

Holweger, H., \& Rentzsch-Holm, I. 1995, A\&A, 303, 819

Holweger, H., Hempel, M., \& Kamp, I. 1999, A\&A, 350, 603

Howe, K. S., \& Clarke, C. J. 2009, MNRAS, 392, 448

Iglesias, D., Bayo, A., Olofsson, J., et al. 2018, MNRAS, 480, 488

Iglesias, D. P., Olofsson, J., Bayo, A., et al. 2019, MNRAS, 490, 5218

Jaschek, M., Jaschek, C., \& Andrillat, Y. 1988, A\&AS, 72, 505

Jura, M. 2015, AJ, 150, 166

Kalas, P. 2005, ApJ, 635, L169

Kasper, M., Apai, D., Wagner, K., \& Robberto, M. 2015, ApJ, 812, L33

Kaufer, A., Stahl, O., Tubbesing, S., et al. 1999, The Messenger, 95, 8

Kausch, W., Noll, S., Smette, A., et al. 2015, A\&A, 576, A78

Kennedy, G. M., \& Wyatt, M. C. 2014, MNRAS, 444, 3164

Kiefer, F., Lecavelier des Etangs, A., Boissier, J., et al. 2014a, Nature, 514, 462

Kiefer, F., Lecavelier des Etangs, A., Augereau, J.-C., et al. 2014b, A\&A, 561, L10

Kirchschlager, F., Wolf, S., Krivov, A. V., Mutschke, H., \& Brunngräber, R. 2017, MNRAS, 467, 1614

Koen, C. 2003, MNRAS, 341, 1385

Kóspál, Á., Moór, A., Juhász, A., et al. 2013, ApJ, 776, 77

Kral, Q., Matrà, L., Wyatt, M. C., \& Kennedy, G. M. 2017, MNRAS, 469, 521

Kral, Q., Marino, S., Wyatt, M. C., Kama, M., \& Matrà, L. 2019, MNRAS, 489, 3670

Lagrange, A.-M., Beust, H., Mouillet, D., et al. 1998, A\&A, 330, 1091

Lagrange, A. M., Desort, M., Galland, F., Udry, S., \& Mayor, M. 2009, A\&A 495,335

Lagrange, A.-M., Bonnefoy, M., Chauvin, G., et al. 2010, Science, 329, 57

Lagrange-Henri, A. M., Beust, H., Ferlet, R., Vidal-Madjar, A., \& Hobbs, L. M 1990a, A\&A, 227, L13

Lagrange-Henri, A. M., Ferlet, R., Vidal-Madjar, A., et al. 1990b, A\&AS, 85, 1089

Lagrange-Henri, A. M., Ferlet, R., Vidal-Madjar, A., \& Beust, H. 1991, A\&A, 246, 507

Lecavelier Des Etangs, A., Deleuil, M., Vidal-Madjar, A., et al. 1997, A\&A, 325, 228

Lieman-Sifry, J., Hughes, A. M., Carpenter, J. M., et al. 2016, ApJ, 828, 25

Lisse, C. M., Sitko, M. L., Russell, R. W., et al. 2017, ApJ, 840, L20

Maldonado, J., \& Villaver, E. 2017, A\&A, 602, A38

Maldonado, J., Eiroa, C., Villaver, E., Montesinos, B., \& Mora, A. 2012, A\&A, 541, A 40 
Maldonado, J., Villaver, E., \& Eiroa, C. 2018, A\&A, 612, A93 Mamajek, E. E., \& Bell, C. P. M. 2014, MNRAS, 445, 2169

Marboeuf, U., Bonsor, A., \& Augereau, J.-C. 2016, Planet. Space Sci., 133, 47 Marino, S., Matrà, L., Stark, C., et al. 2016, MNRAS, 460, 2933

Marino, S., Wyatt, M. C., Panić, O., et al. 2017, MNRAS, 465, 2595

Marino, S., Bonsor, A., Wyatt, M. C., \& Kral, Q. 2018, MNRAS, 479, 1651

Matrà, L., Dent, W. R. F., Wyatt, M. C., et al. 2017, MNRAS, 464, 1415

Matthews, B. C., Krivov, A. V., Wyatt, M. C., Bryden, G., \& Eiroa, C. 2014, Protostars and Planets VI, 521

Mayor, M., \& Queloz, D. 1995, Nature, 378, 355

Mékarnia, D., Chapellier, E., Guillot, T., et al. 2017, A\&A, 608, L6

Mellon, S. N., Mamajek, E. E., Zwintz, K., et al. 2019, ApJ, 870, 36

Montesinos, B., Eiroa, C., Lillo-Box, J., et al. 2019, A\&A, 629, A19

Montgomery, S. L., \& Welsh, B. Y. 2012, PASP, 124, 1042

Montgomery, S. L., \& Welsh, B. Y. 2017, MNRAS, 468, L55

Moór, A., Pascucci, I., Kóspál, Á., et al. 2011a, ApJS, 193, 4

Moór, A., Ábrahám, P., Juhász, A., et al. 2011b, ApJ, 740, L7

Moór, A., Henning, T., Juhász, A., et al. 2015a, ApJ, 814, 42

Moór, A., Kóspál, Á., Ábrahám, P., et al. 2015b, MNRAS, 447, 577

Moór, A., Curé, M., Kóspál, Á., et al. 2017, ApJ, 849, 123

Mora, A., Merín, B., Solano, E., et al. 2001, A\&A, 378, 116

Morales, F. Y., Bryden, G., Werner, M. W., \& Stapelfeldt, K. R. 2016, ApJ, 831, 97

Murphy, S. J., \& Paunzen, E. 2017, MNRAS, 466, 546

Murphy, S. J., Corbally, C. J., Gray, R. O., et al. 2015, PASA, 32, e036

Nesvorný, D., Jenniskens, P., Levison, H. F., et al. 2010, ApJ, 713, 816

Nielsen, M. B., Gizon, L., Schunker, H., \& Karoff, C. 2013, A\&A, 557, L10

Nuñez, P. D., Scott, N. J., Mennesson, B., et al. 2017, A\&A, 608, A113

Paunzen, E. 2004, The A-Star Puzzle, eds. J. Zverko, J. Ziznovsky, S. J. Adelman, \& W. W. Weiss, IAU Symp., 224, 443

Pawellek, N., Krivov, A. V., Marshall, J. P., et al. 2014, ApJ, 792, 65

Pecaut, M. J., \& Mamajek, E. E. 2013, ApJS, 208, 9

Pecaut, M. J., \& Mamajek, E. E. 2016, MNRAS, 461, 794

Pecaut, M. J., Mamajek, E. E., \& Bubar, E. J. 2012, ApJ, 746, 154

Plavchan, P., Werner, M. W., Chen, C. H., et al. 2009, ApJ, 698, 1068

Quirrenbach, A., Amado, P. J., Caballero, J. A., et al. 2016, Ground-based and Airborne Instrumentation for Astronomy VI, Proc. SPIE, 9908, 990812

Rappaport, S., Vanderburg, A., Jacobs, T., et al. 2018, MNRAS, 474, 1453

Raskin, G., van Winckel, H., Hensberge, H., et al. 2011, A\&A, 526, A69

Rebollido, I., Eiroa, C., Montesinos, B., et al. 2018, A\&A, 614, A3

Rebollido, I., Eiroa, C., Montesinos, B., et al. 2019, A\&A, 625, C2

Redfield, S. 2007, ApJ, 656, L97

Redfield, S., \& Linsky, J. L. 2008, ApJ, 673, 283
Redfield, S., Kessler-Silacci, J. E., \& Cieza, L. A. 2007, ApJ, 661, 944 Riviere-Marichalar, P., Barrado, D., Augereau, J.-C., et al. 2012, A\&A, 546, L8 Riviere-Marichalar, P., Pinte, C., Barrado, D., et al. 2013, A\&A, 555, A67 Riviere-Marichalar, P., Barrado, D., Montesinos, B., et al. 2014, A\&A, 565, A68 Roberge, A., \& Weinberger, A. J. 2008, ApJ, 676, 509

Roberge, A., Feldman, P. D., Weinberger, A. J., Deleuil, M., \& Bouret, J.-C. 2006, Nature, 441, 724

Roberge, A., Kamp, I., Montesinos, B., et al. 2013, ApJ, 771, 69

Roberge, A., Welsh, B. Y., Kamp, I., Weinberger, A. J., \& Grady, C. A. 2014, ApJ, 796, L11

Rodriguez, E., Lopez de Coca, P., Rolland, A., Garrido, R., \& Costa, V. 1994, A\&AS, 106, 21

Schmitt, J. H. M. M., Schröder, K.-P., Rauw, G., et al. 2014, Astron. Nachr., 335, 787

Schüppler, C., Krivov, A. V., Löhne, T., et al. 2016, MNRAS, 461, 2146

Sezestre, É., Augereau, J. C., \& Thébault, P. 2019, A\&A, 626, A2

Sibthorpe, B., Kennedy, G. M., Wyatt, M. C., et al. 2018, MNRAS, 475, 3046

Smette, A., Sana, H., Noll, S., et al. 2015, A\&A, 576, A77

Somerville, W. B. 1988, The Observatory, 108, 44

Su, K. Y. L., Rieke, G. H., Stansberry, J. A., et al. 2006, ApJ, 653, 675

Thébault, P., \& Beust, H. 2001, A\&A, 376, 621

Thureau, N. D., Greaves, J. S., Matthews, B. C., et al. 2014, MNRAS, 445, 2558 Tokovinin, A. 2008, MNRAS, 389, 925

Torres, C. A. O., Quast, G. R., Melo, C. H. F., \& Sterzik, M. F. 2008, Young Nearby Loose Associations, B. Reipurth, 757

Vican, L., Schneider, A., Bryden, G., et al. 2016, ApJ, 833, 263

Vidal-Madjar, A., Lagrange-Henri, A.-M., Feldman, P. D., et al. 1994, A\&A, 290, 245

Vidal-Madjar, A., Kiefer, F., Lecavelier des Etangs, A., et al. 2017, A\&A, 607, A25

Welsh, B. Y., \& Montgomery, S. 2013, PASP, 125, 759

Welsh, B. Y., \& Montgomery, S. L. 2015, Adv. Astron., 2015, 980323

Welsh, B. Y., \& Montgomery, S. L. 2018, MNRAS, 474, 1515

Welsh, B. Y., \& Montgomery, S. L. 2019, Res. Notes Am. Astron. Soc., 3, 25

Welsh, B. Y., Craig, N., Crawford, I. A., \& Price, R. J. 1998, A\&A, 338, 674

Welsh, B. Y., Lallement, R., Vergely, J.-L., \& Raimond, S. 2010, A\&A, 510, A54

Welty, D. E., Hobbs, L. M., \& Kulkarni, V. P. 1994, ApJ, 436, 152

Welty, D. E., Morton, D. C., \& Hobbs, L. M. 1996, ApJS, 106, 533

Wood, H. J., \& Hollis, J. M. 1971, A\&A, 12, 468

Zieba, S., Zwintz, K., Kenworthy, M. A., \& Kennedy, G. M. 2019, A\&A, 625, L13

Zuckerman, B. 2019, ApJ, 870, 27

Zuckerman, B., \& Song, I. 2012, ApJ, 758, 77 


\section{Appendix A: Narrow stable absorptions}

HD 256
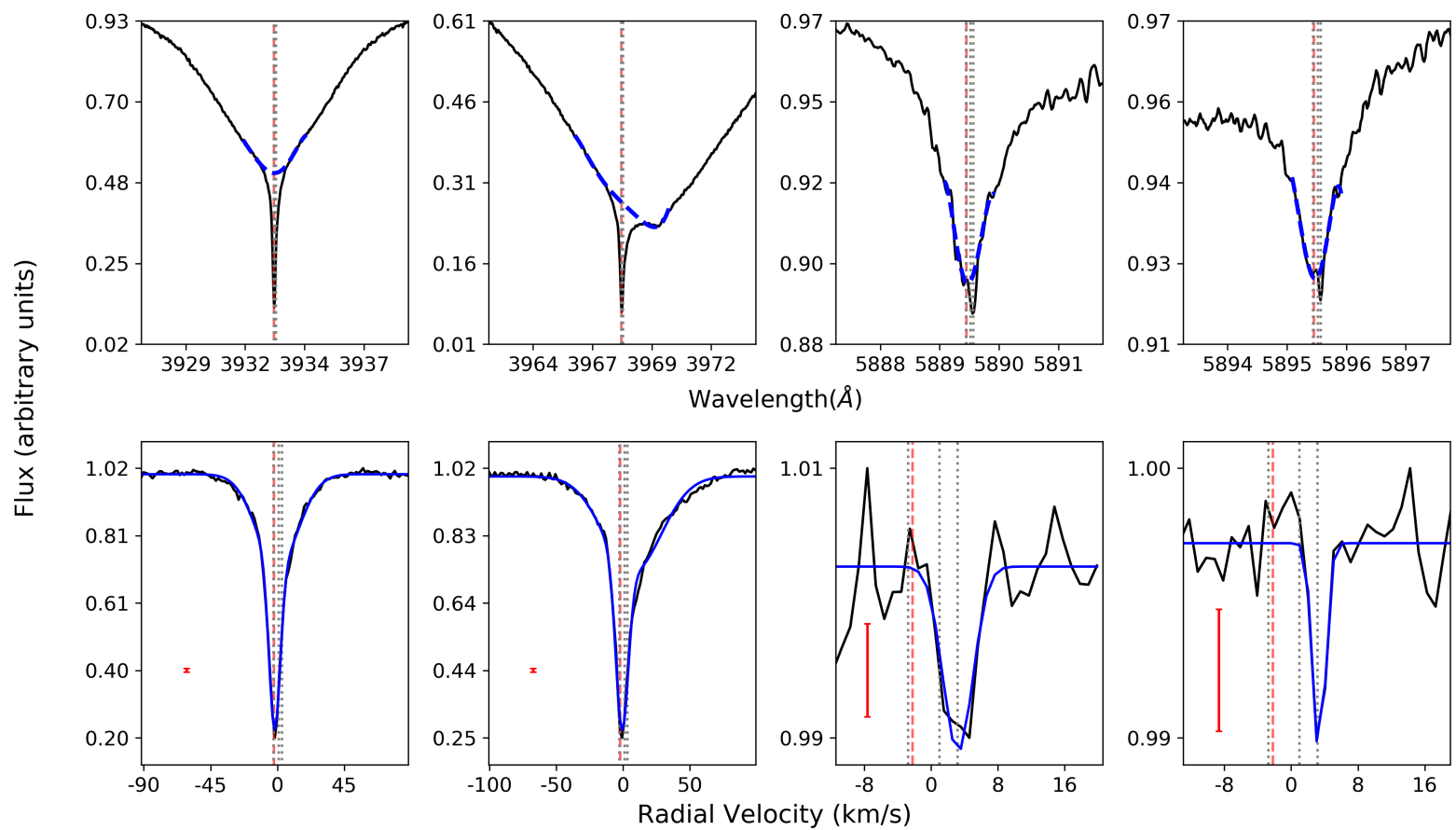

HD 2884
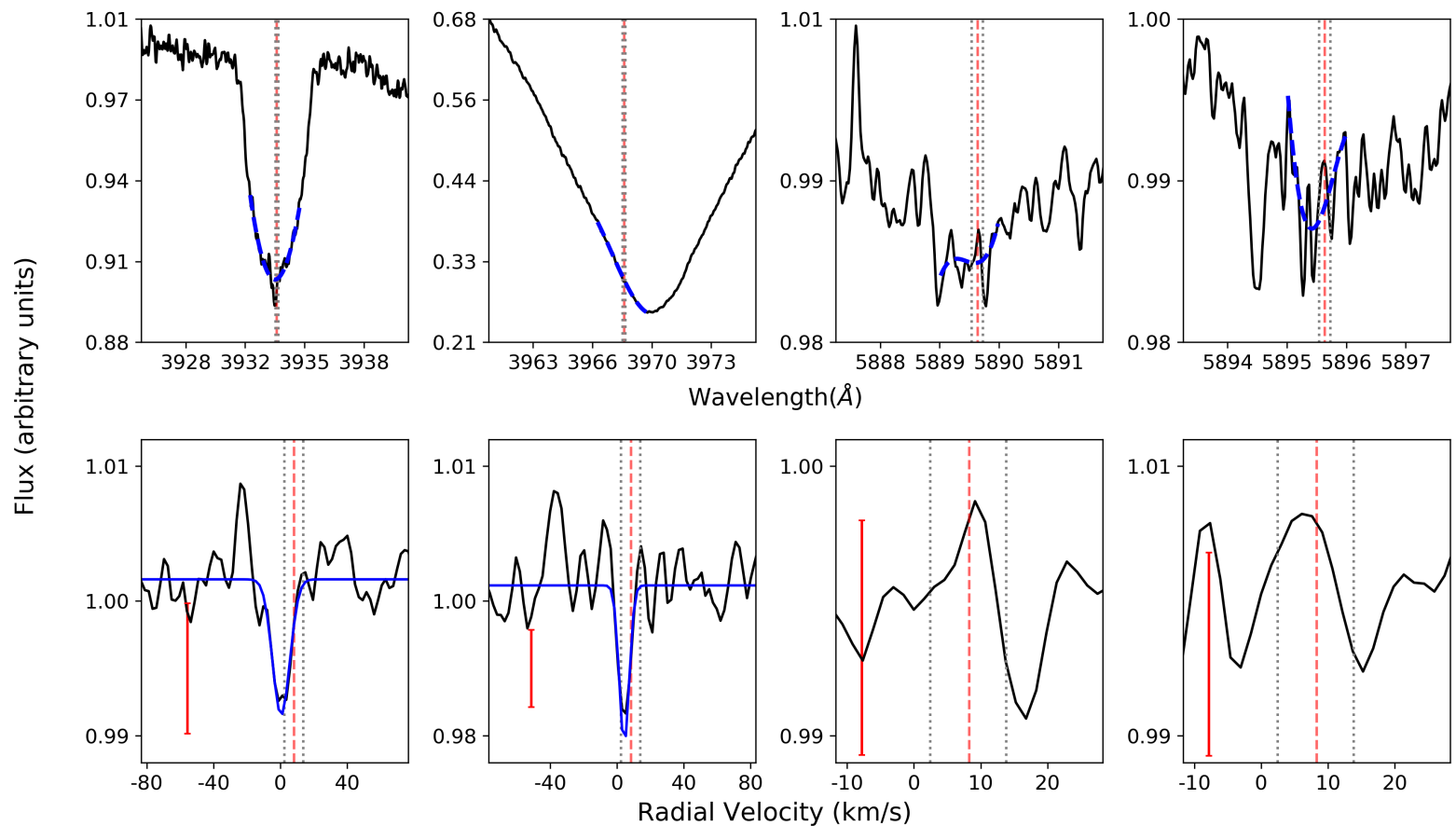

Fig. A.1. Stars showing narrow non-photospheric absorptions. Top panels: photospheric Ca II H \& K and Na I D lines with fitted modelling, the blue-dashed line, $x$-axis shows the wavelength. Bottom panels: residuals once the spectrum was divided by the photosphere, $\mathrm{x}$-axis in velocity. Blue lines mark the fits to the non-photospheric absorptions. Vertical red-dashed and grey dotted-lines represent the stellar radial velocity and the ISM velocities, respectively. The red error bars show three sigma values measured in the continuum adjacent to the photospheric line. 
HD 2885
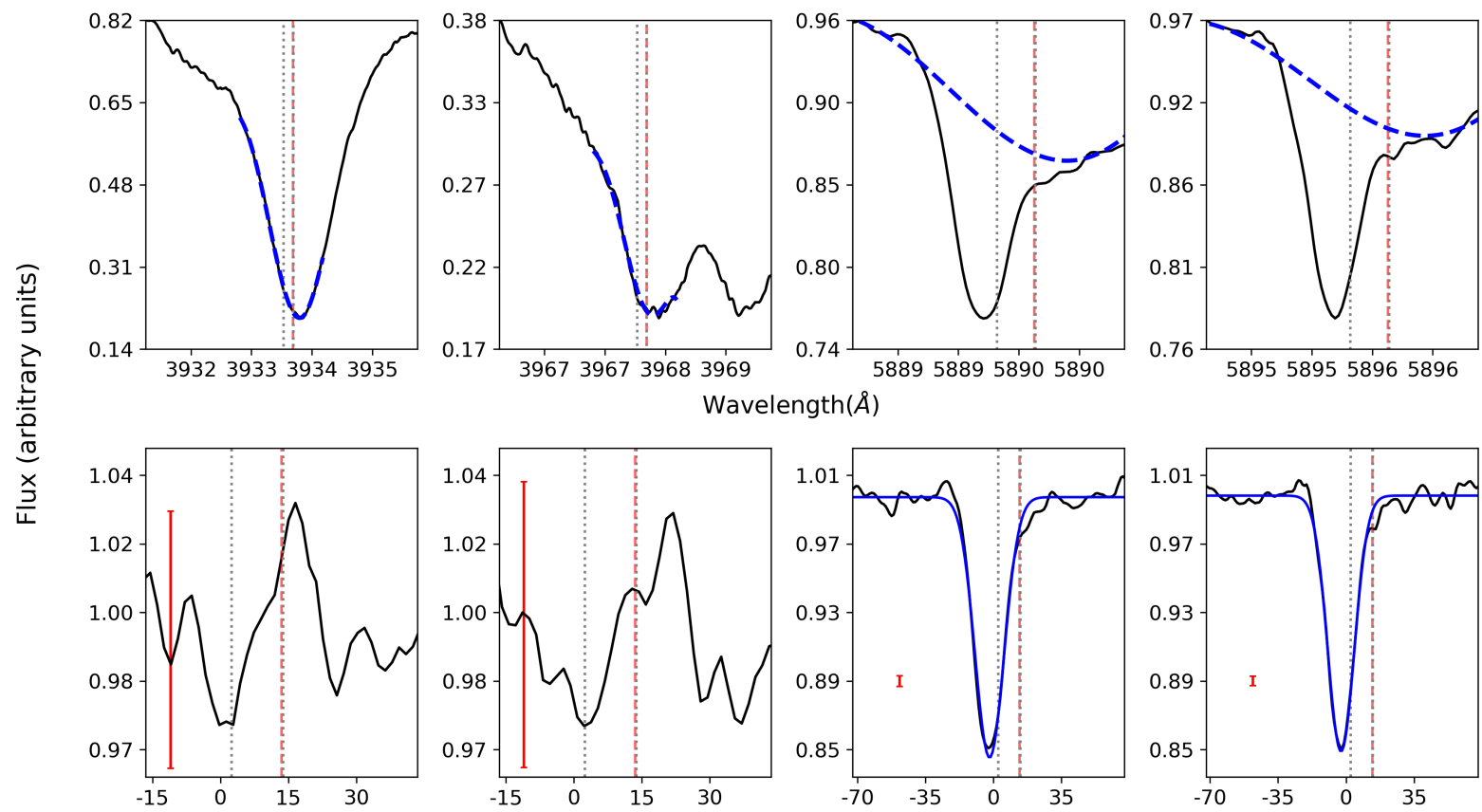

Wavelength $(\AA)$
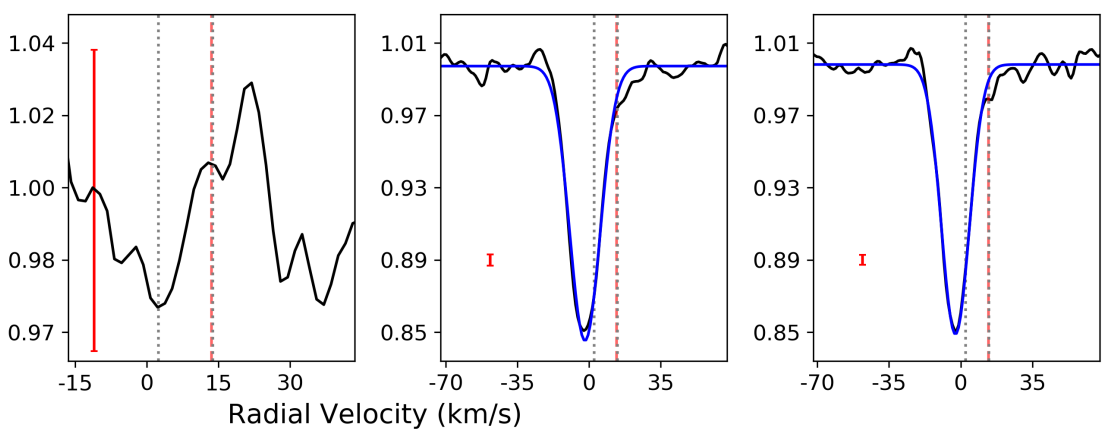

HD 3003
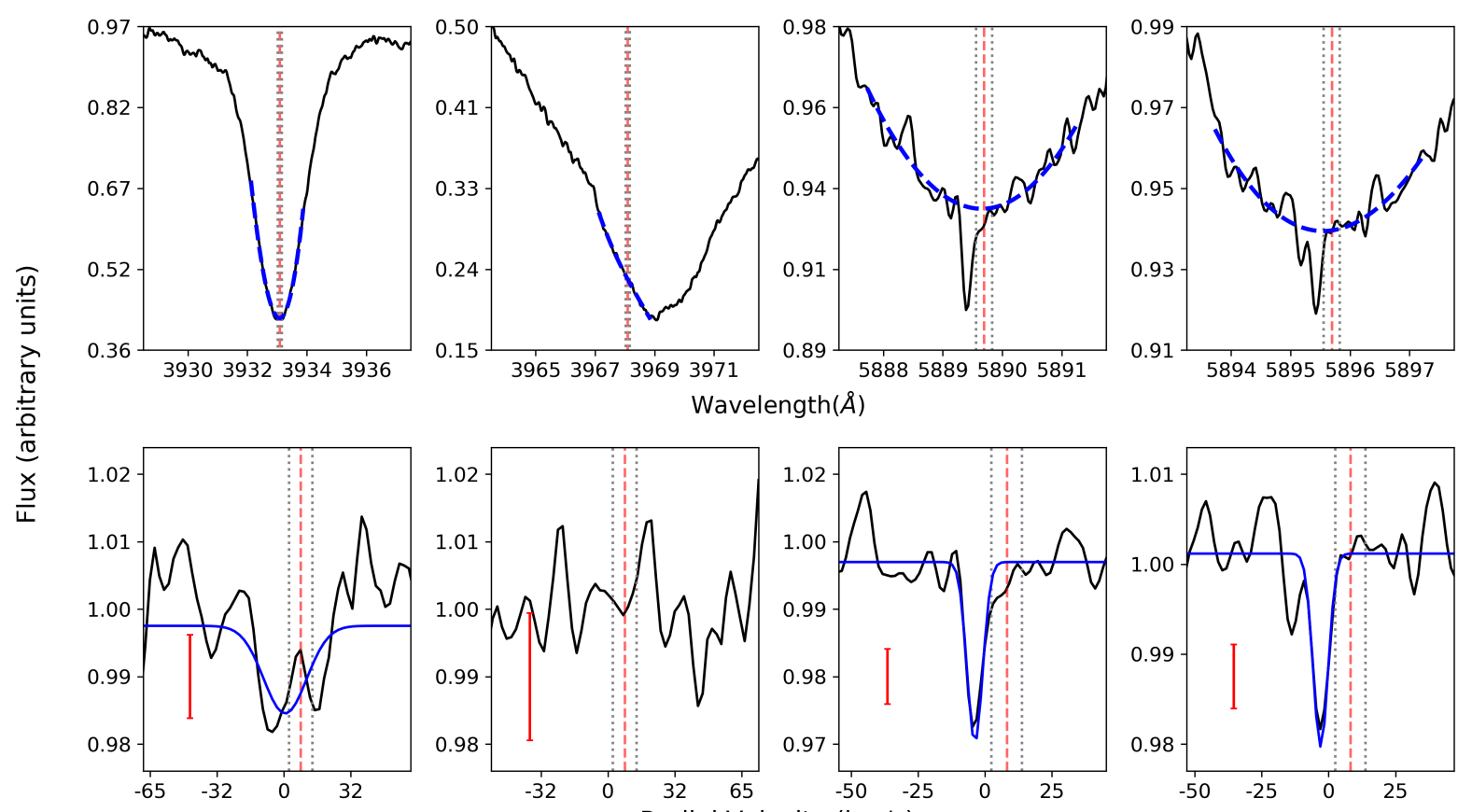

Wavelength $(\AA)$
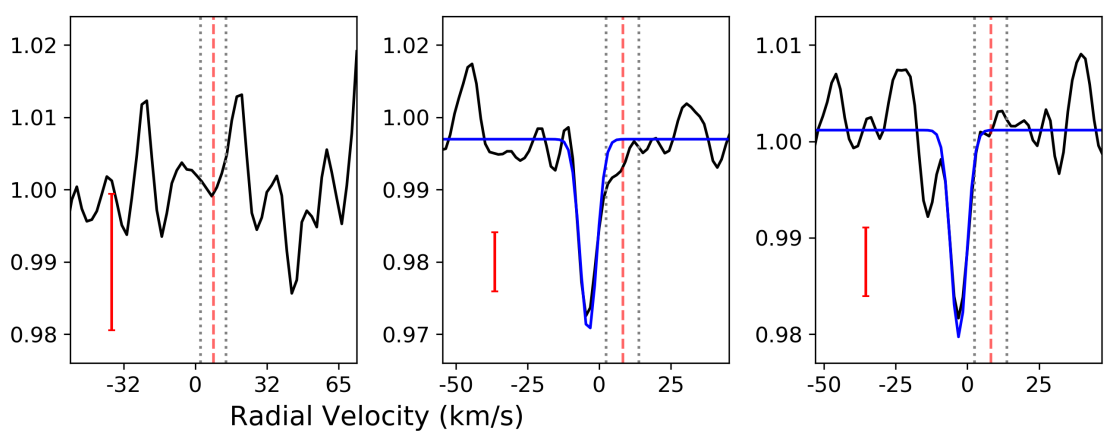

Fig. A.1. continued. 
I. Rebollido et al.: Exocomets: A spectroscopic survey

HD 5267
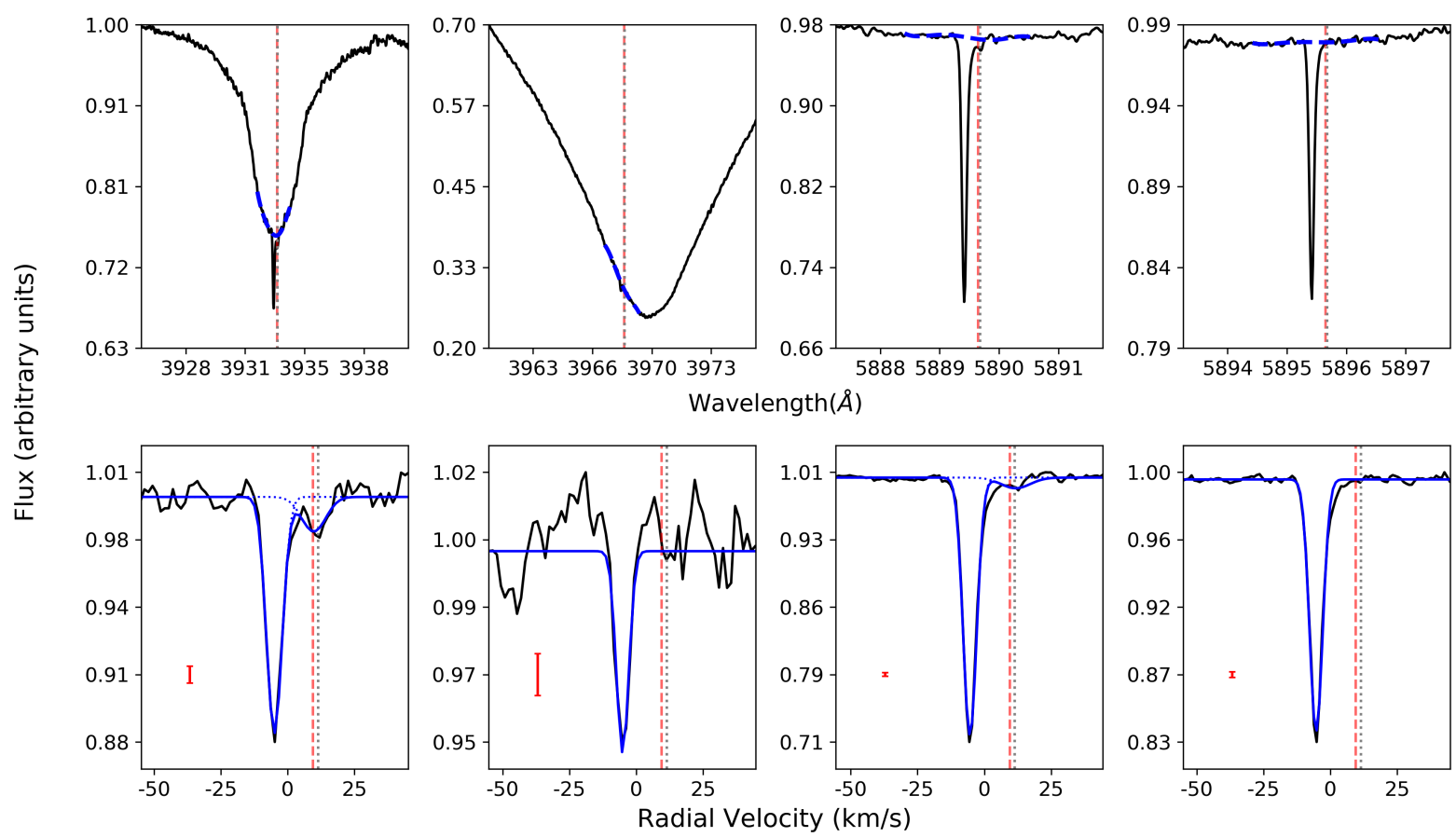

HD 9672
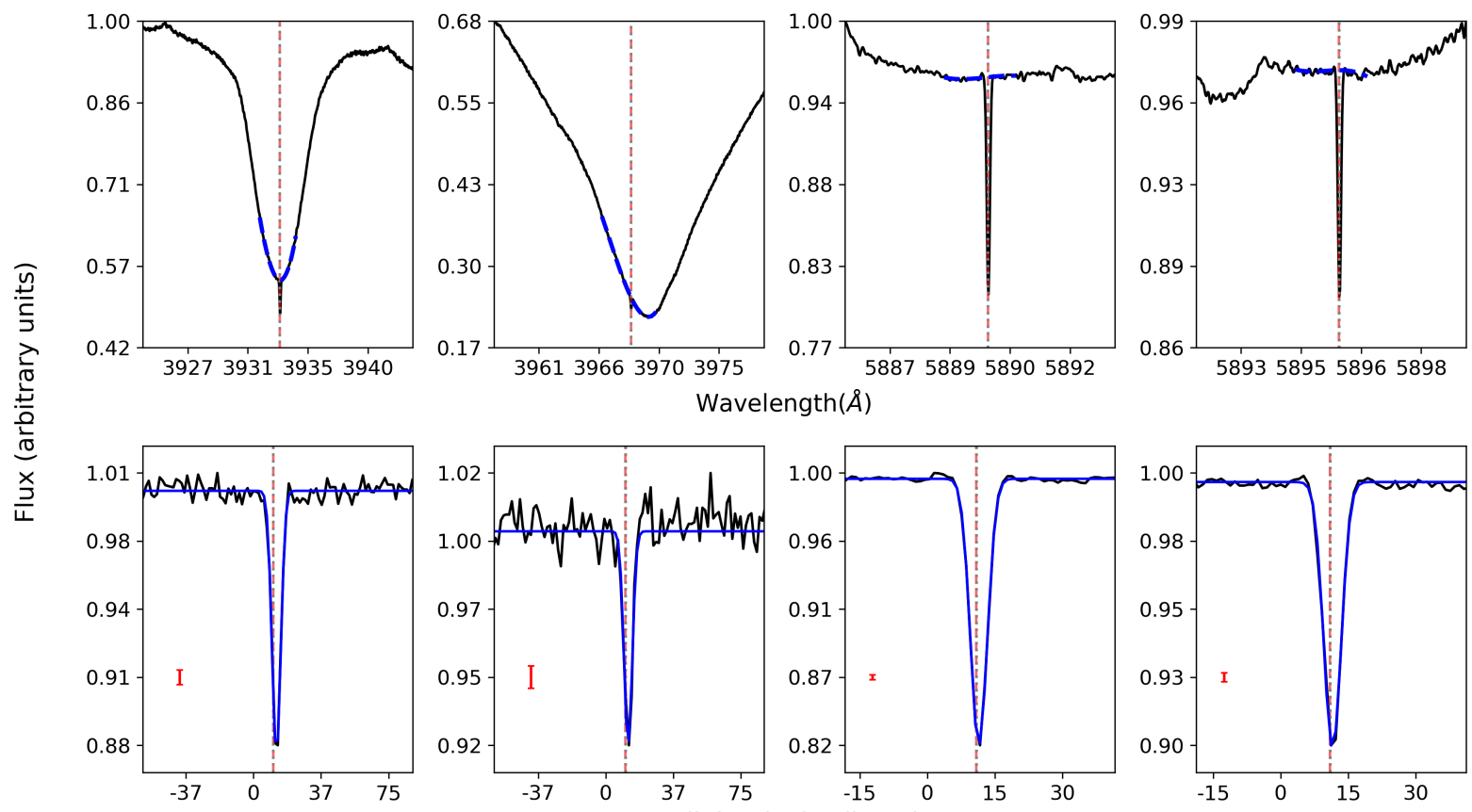
Wavelength $(\AA)$
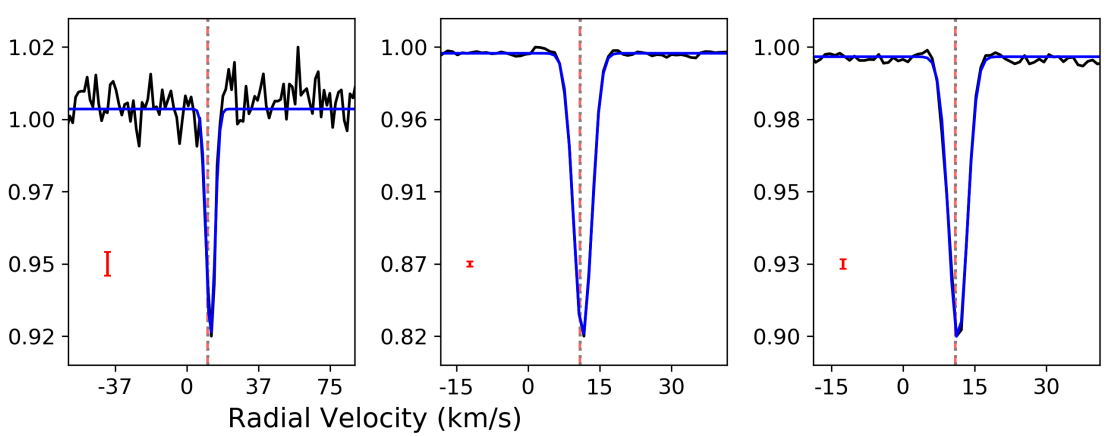

Fig. A.1. continued. 
HD 16978
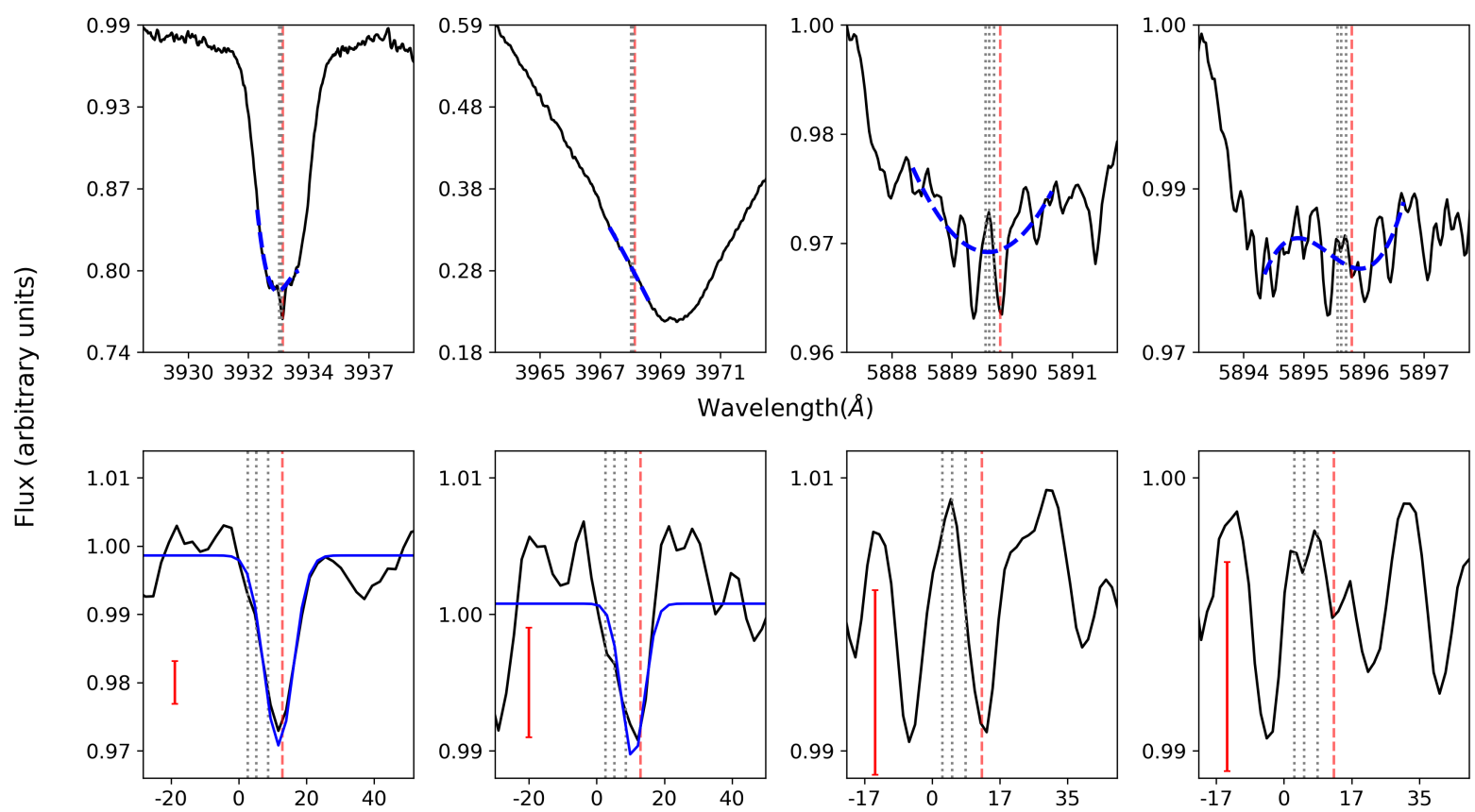
Wavelength $(\AA)$
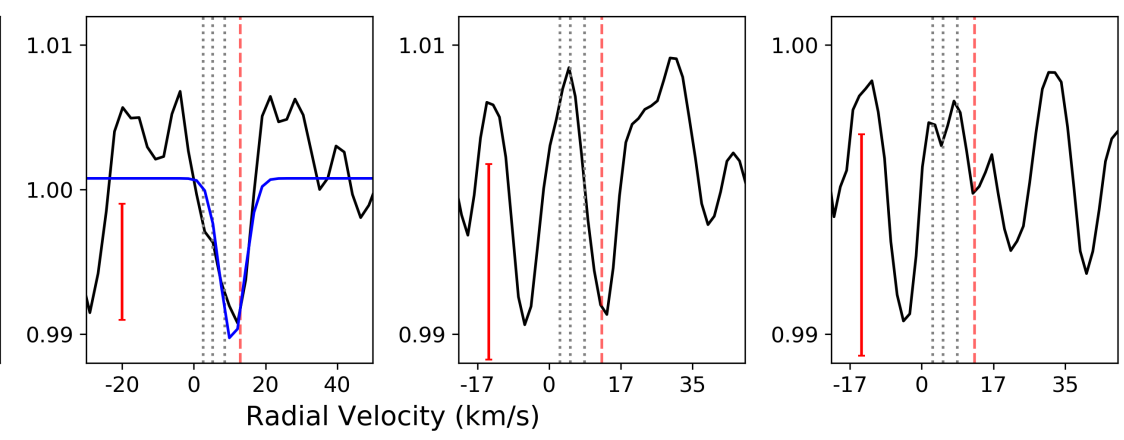

HD 21688
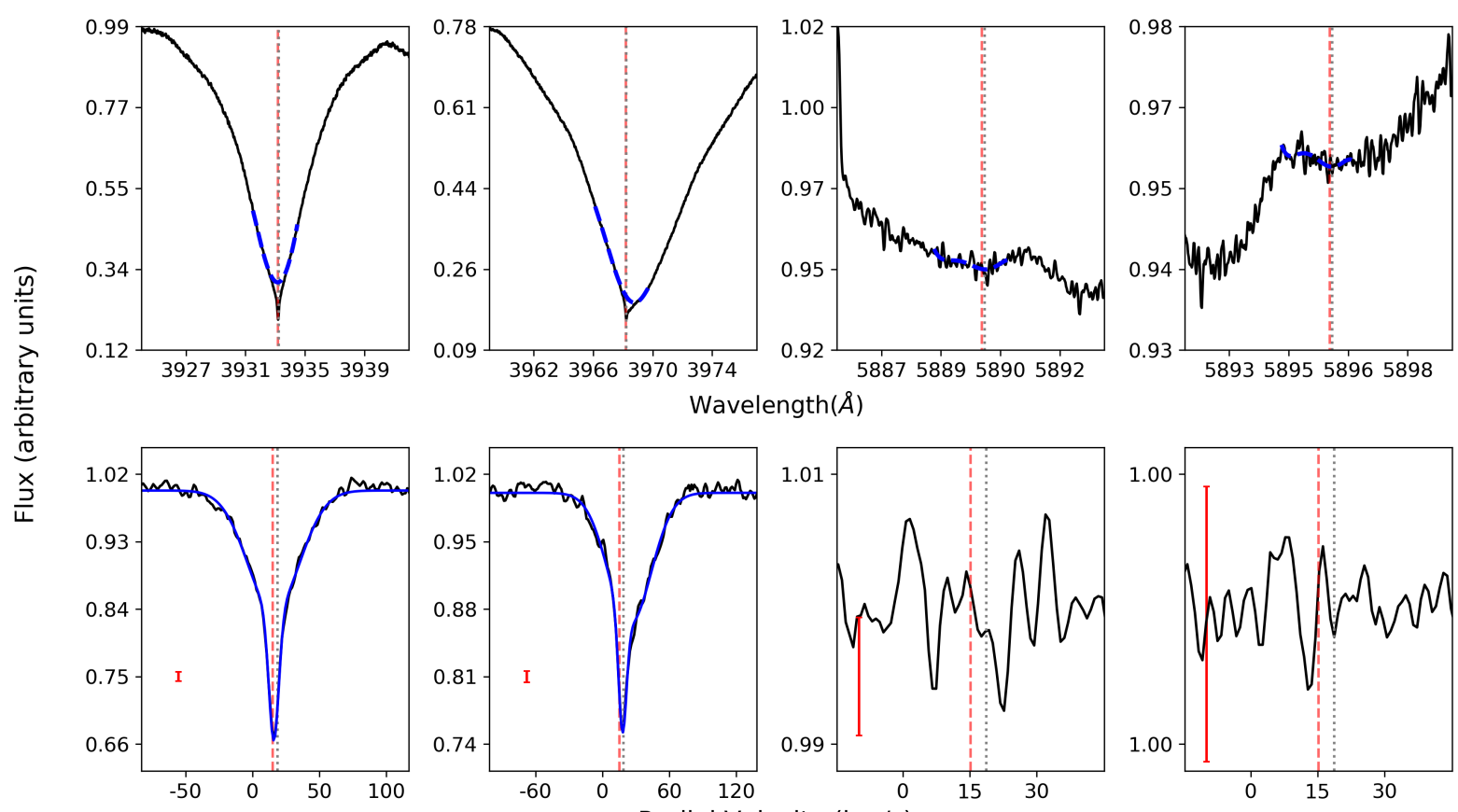
Wavelength $(\AA)$
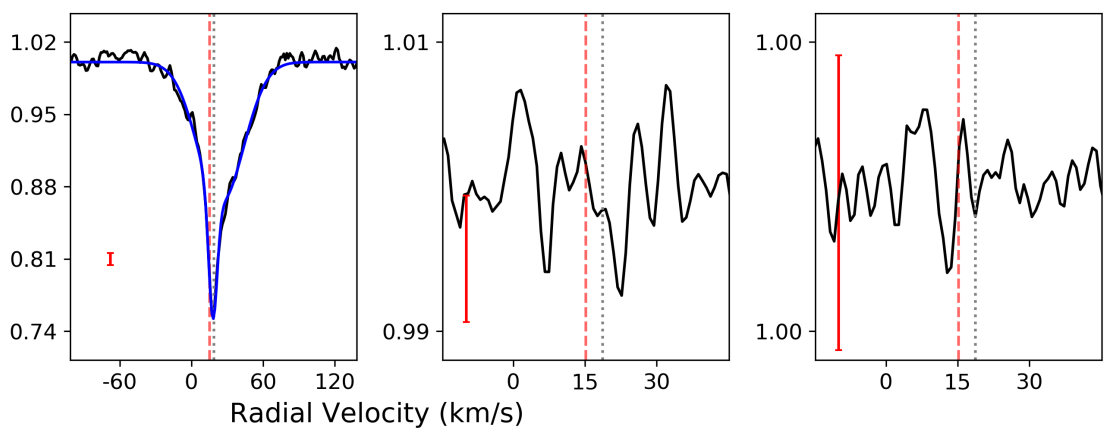

Fig. A.1. continued. 
I. Rebollido et al.: Exocomets: A spectroscopic survey

HD 21620
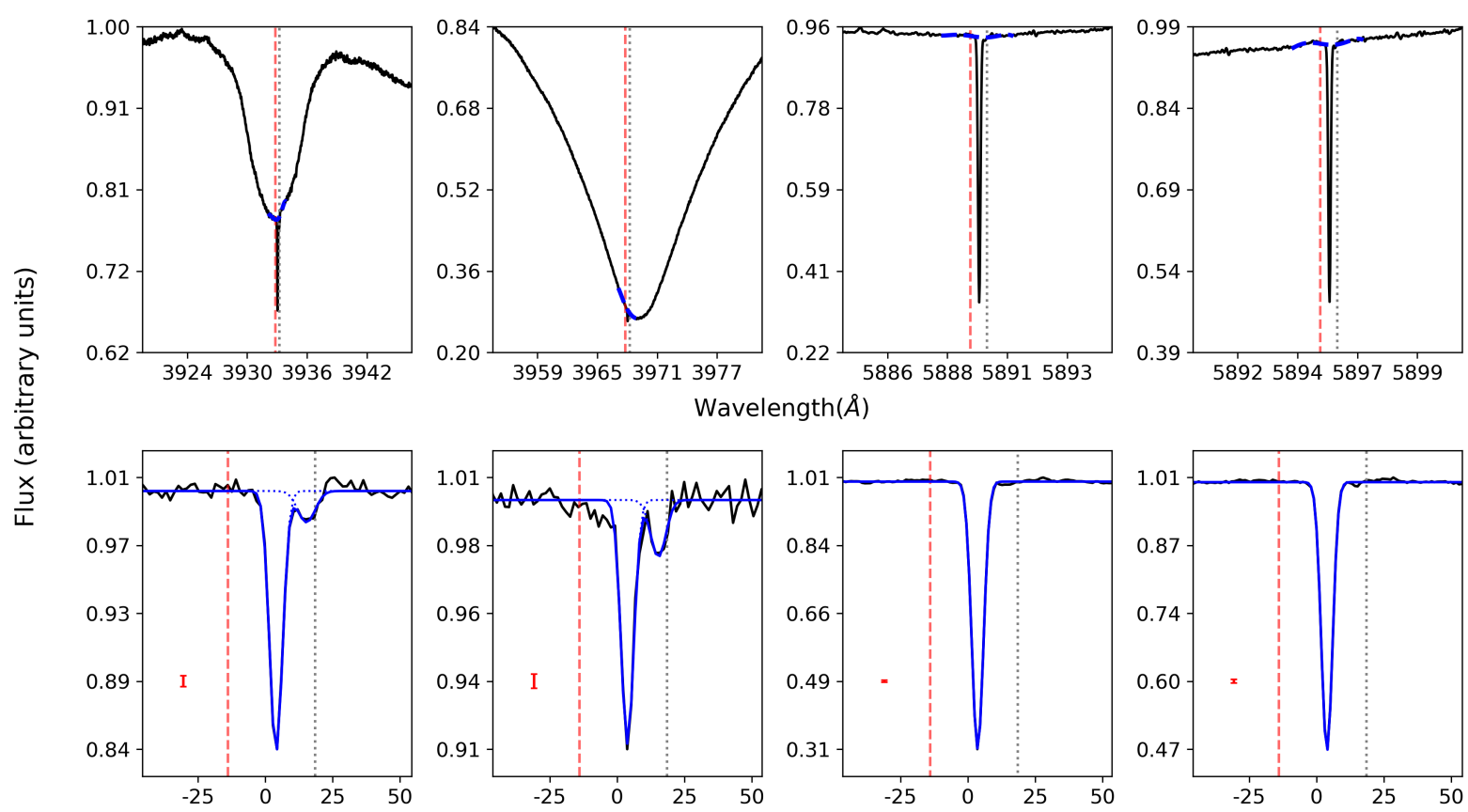

Wavelength $(\AA)$
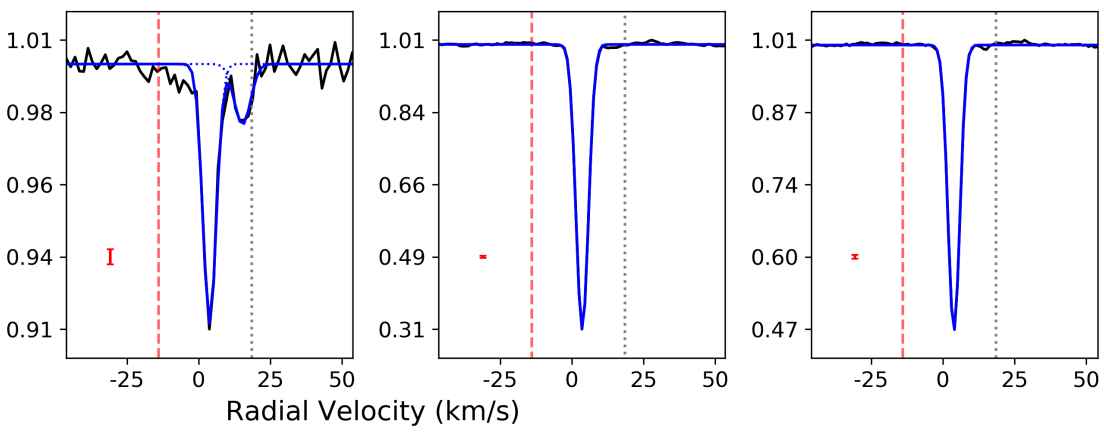

HD 32297
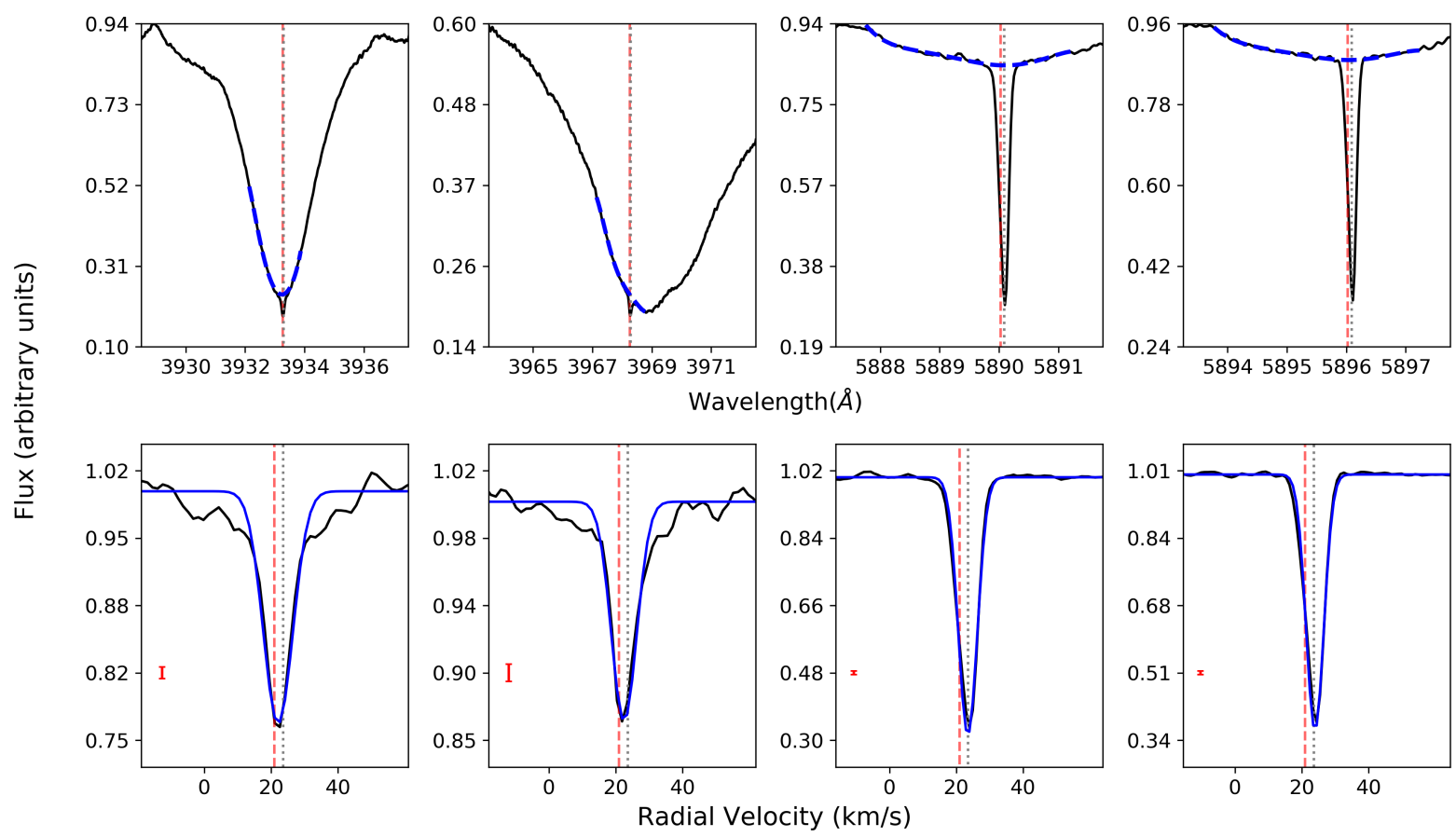

Fig. A.1. continued. 
HD 36546
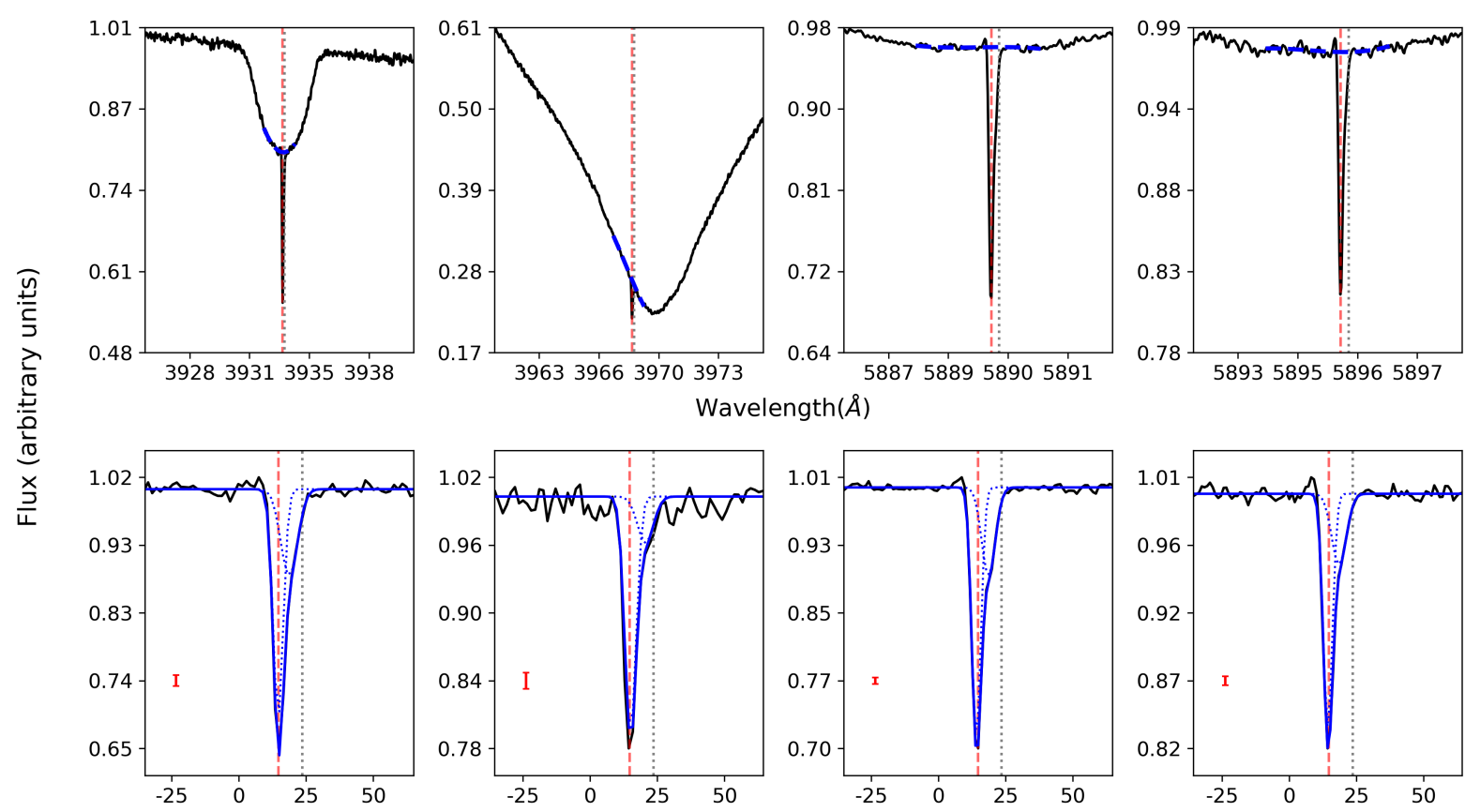

Wavelength $(\AA)$
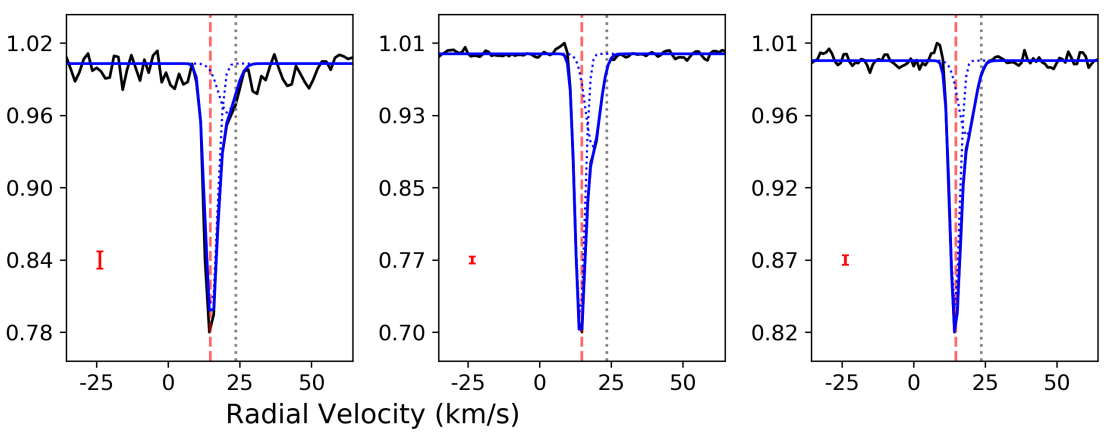

HD 37306
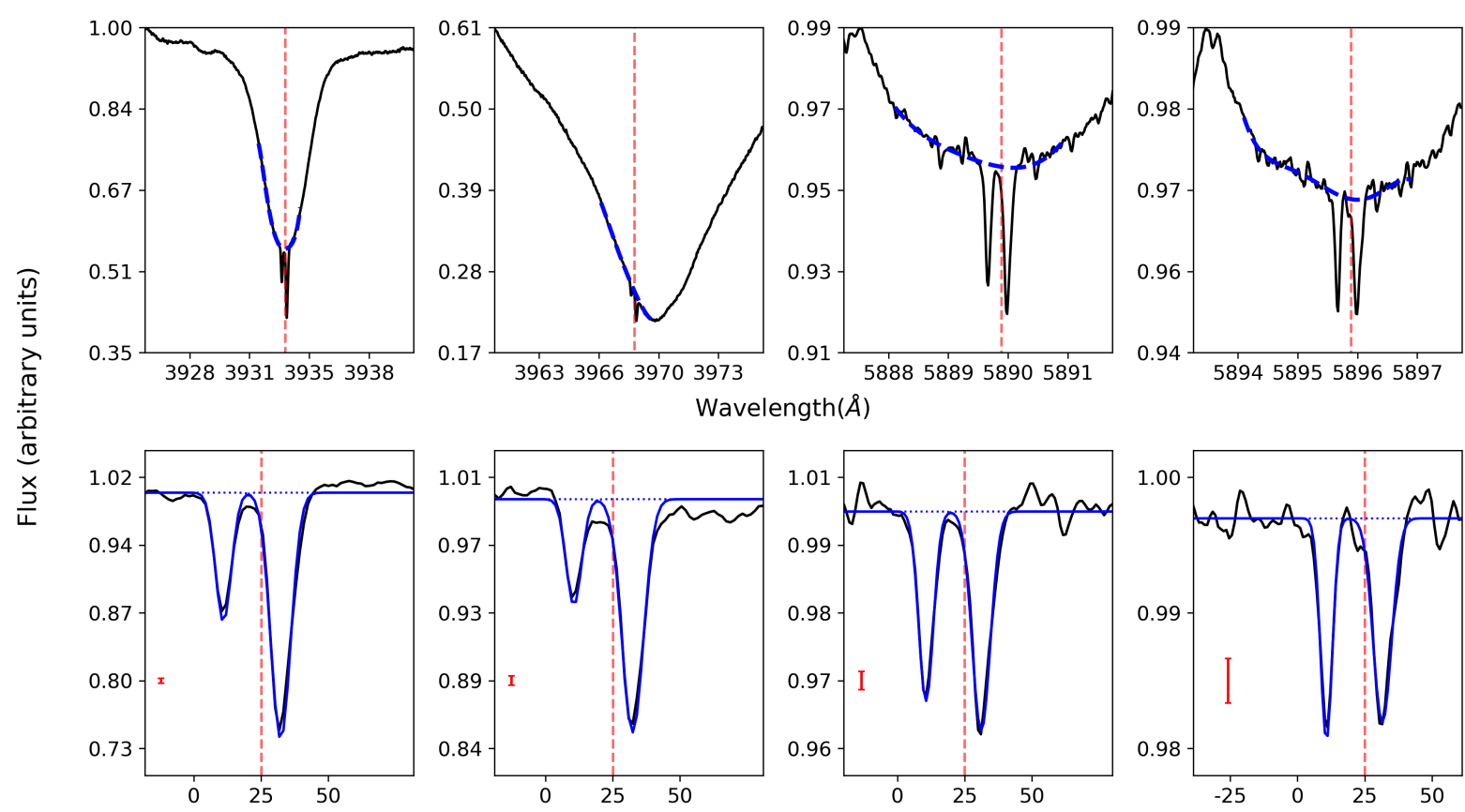

Wavelength $(\AA)$
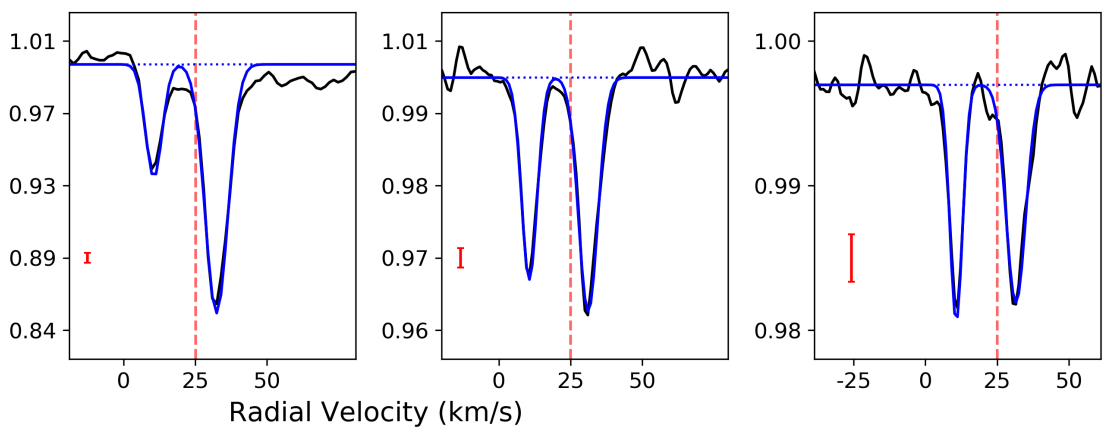

Fig. A.1. continued. 
HD 39182
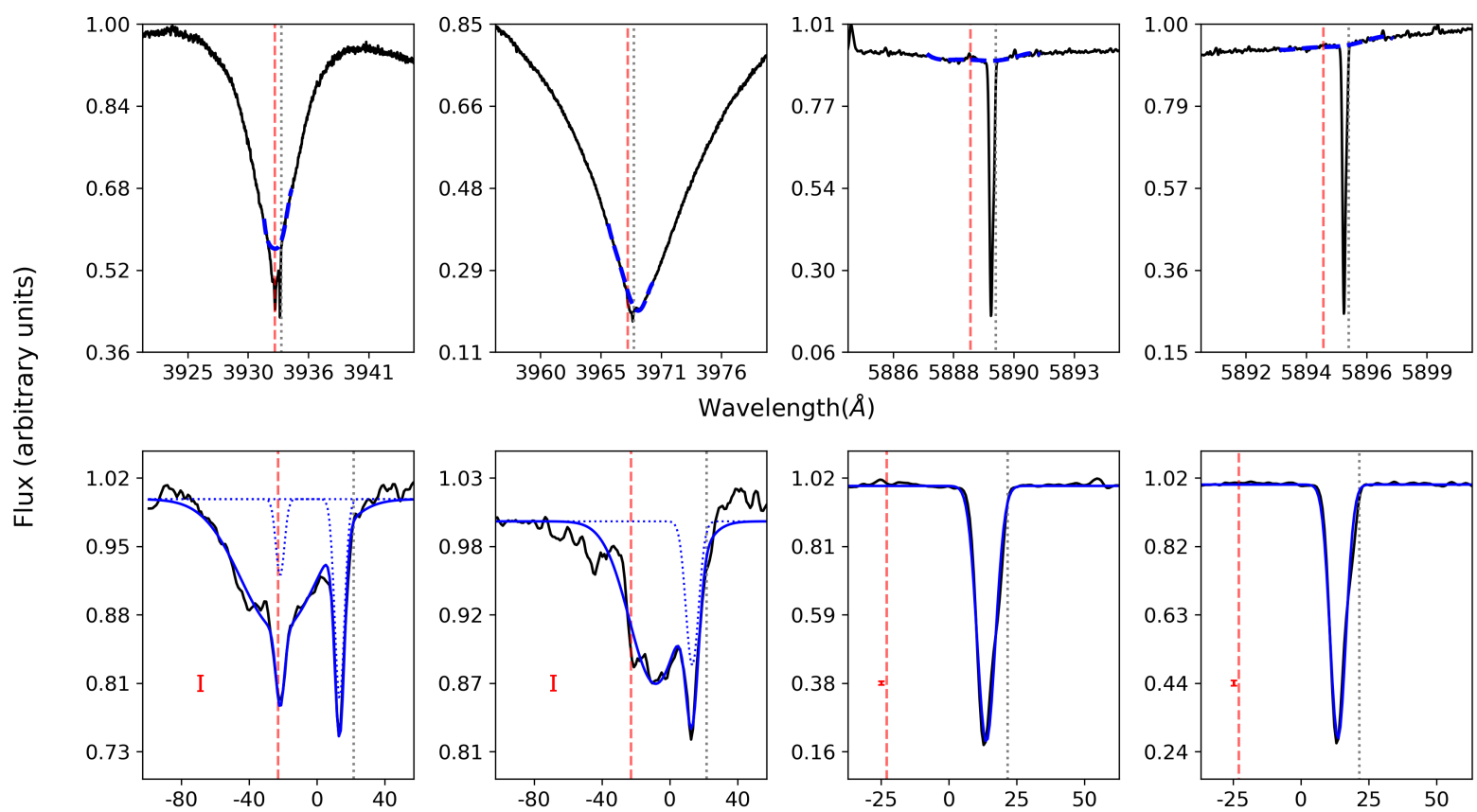

Wavelength $(\AA)$
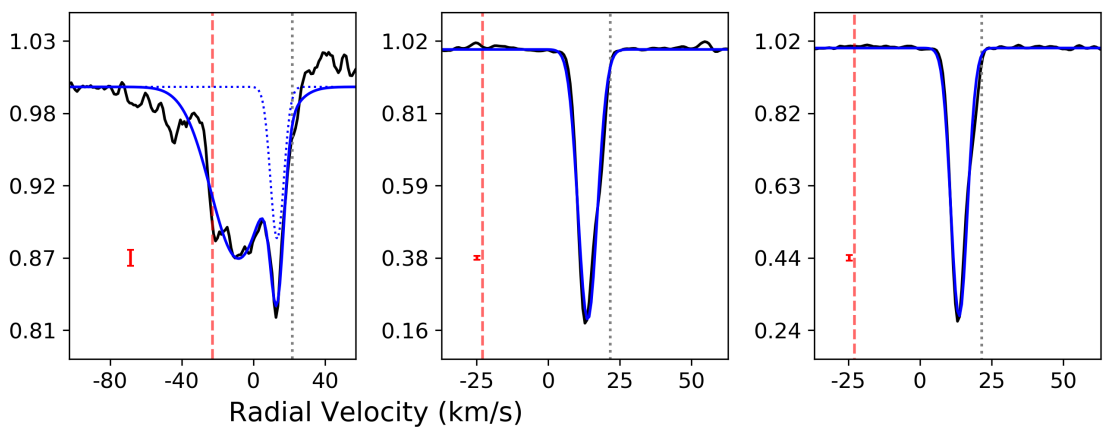

HD 42111
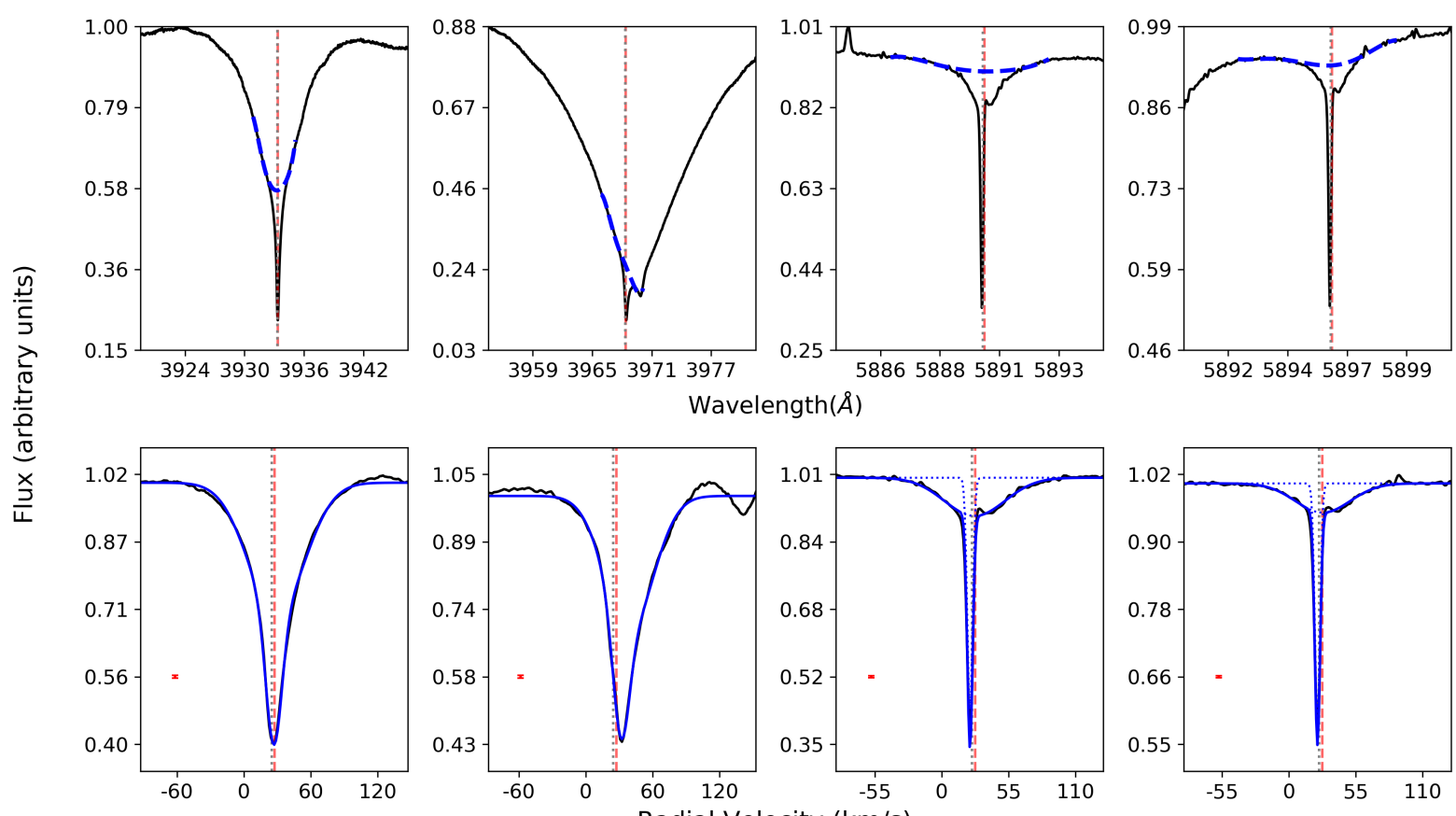

Wavelength $(\AA)$
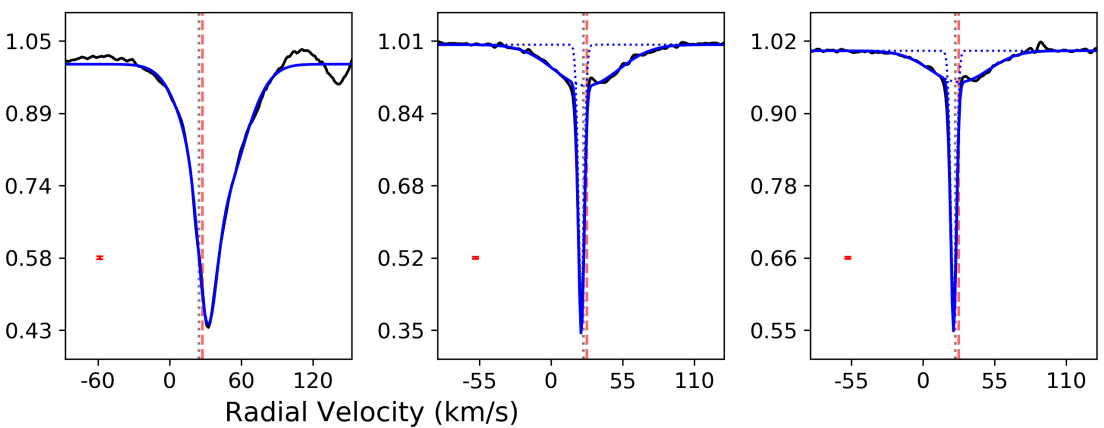

Fig. A.1. continued. 
HD 50241
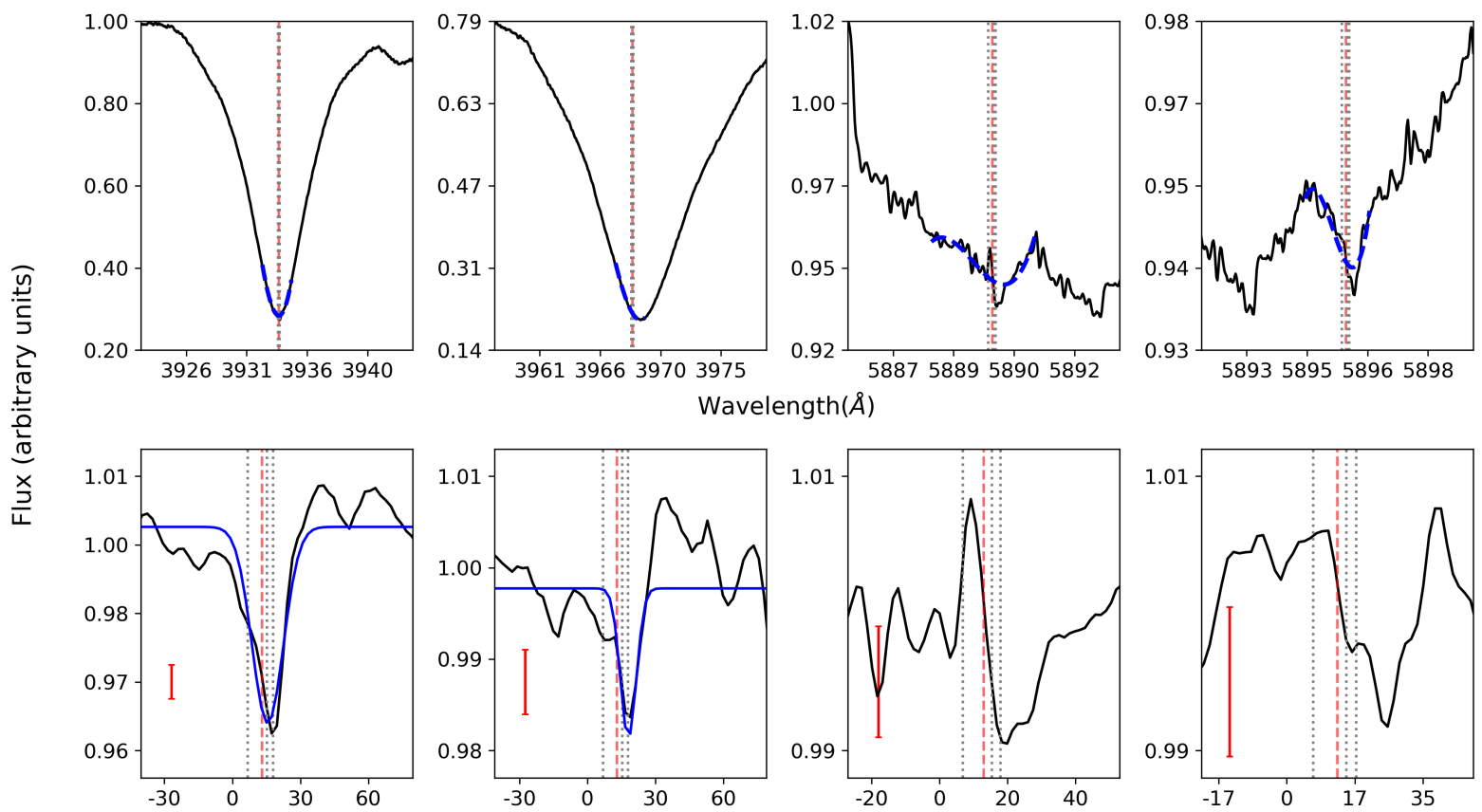
Wavelength $(\AA)$
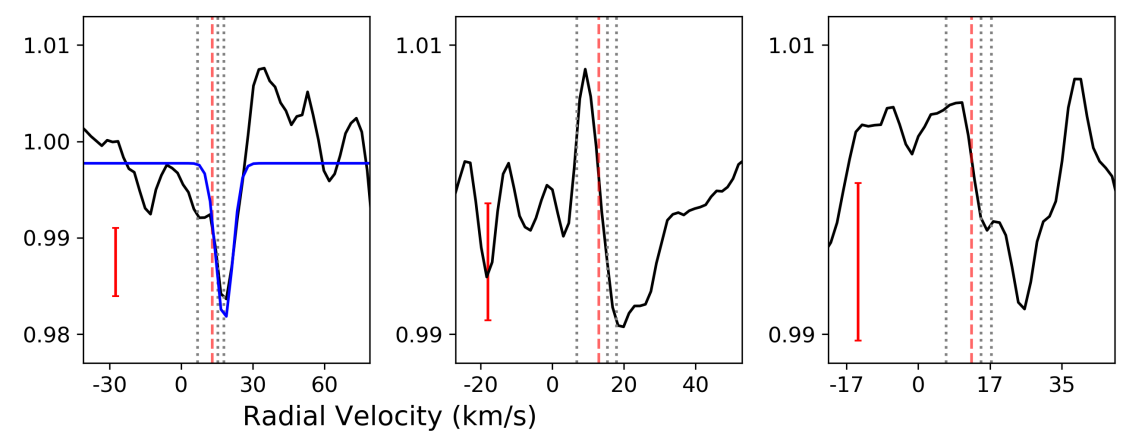

HD 71043
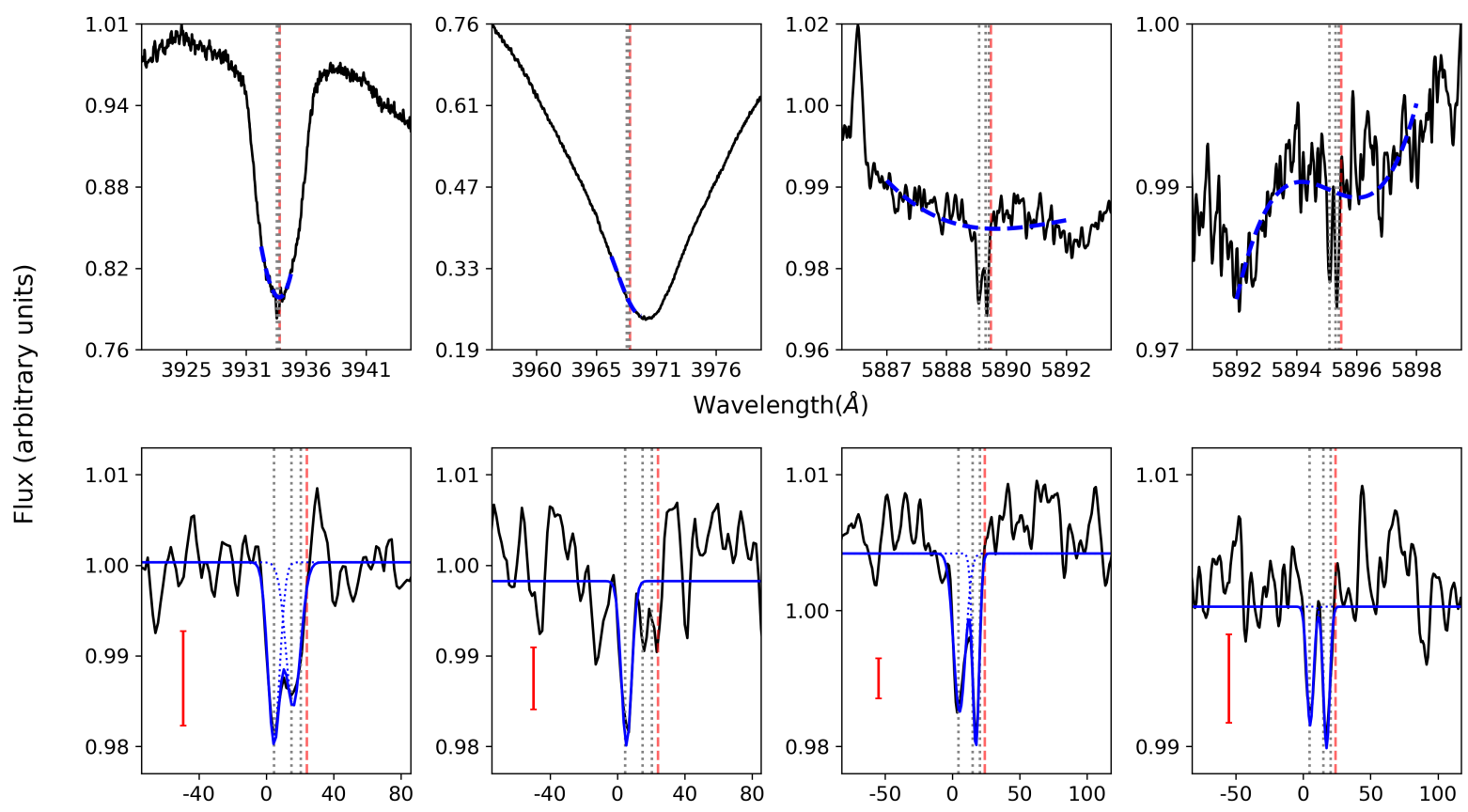
Wavelength $(\AA$
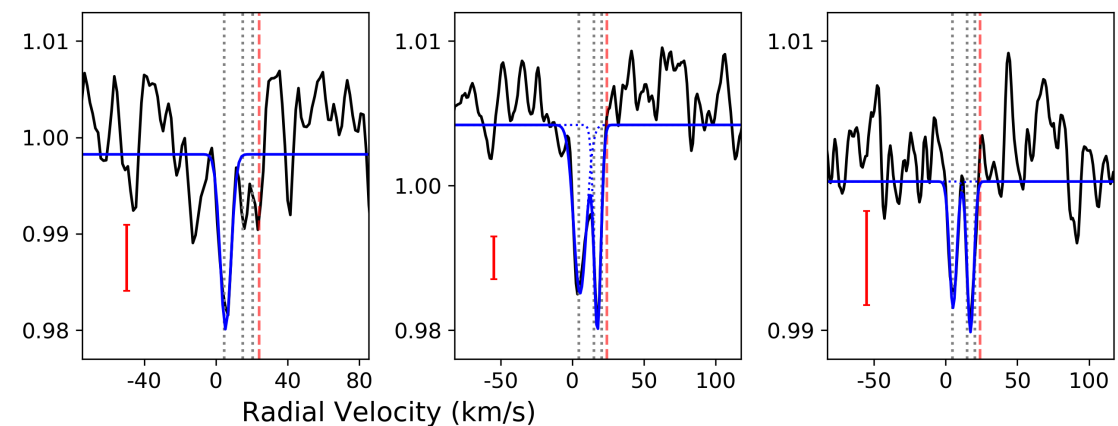

Fig. A.1. continued. 

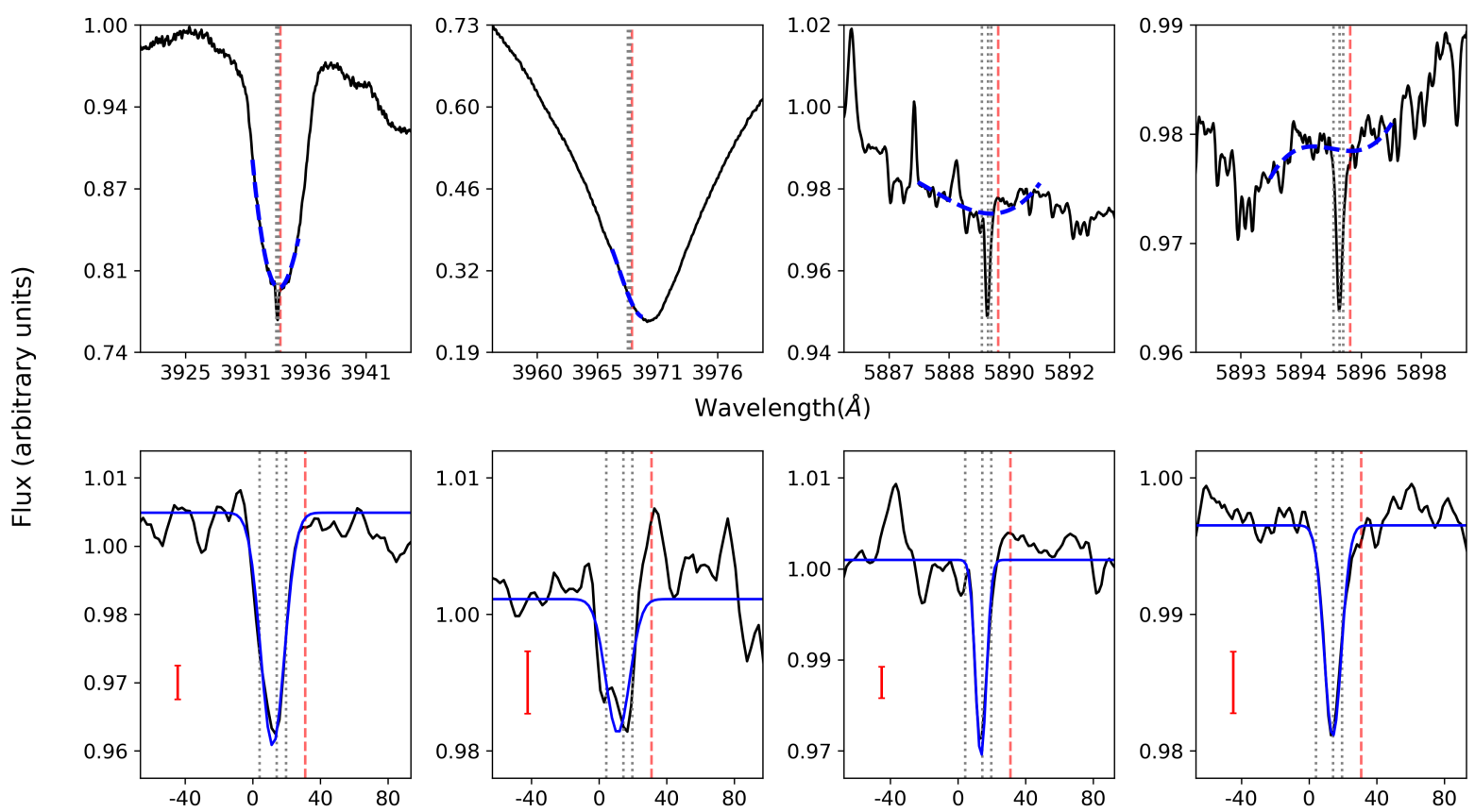
Wavelength $(\AA)$
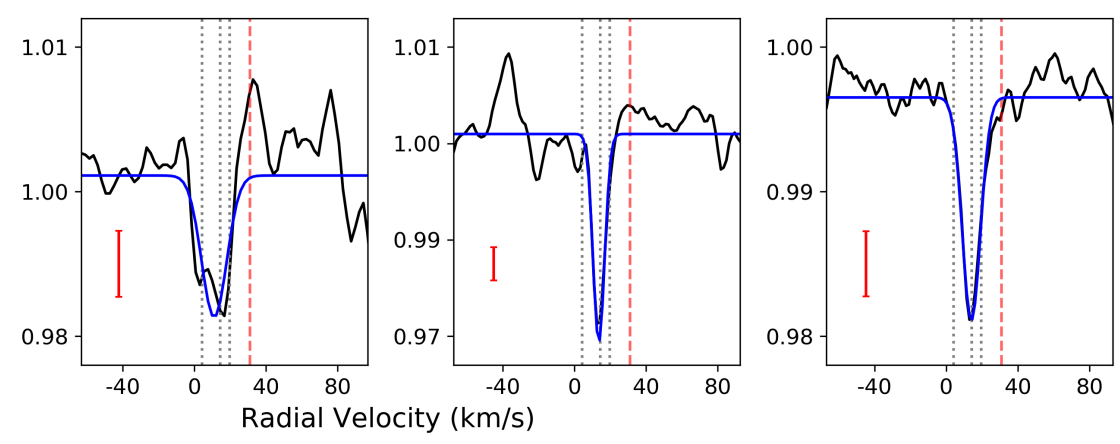

HD 80007
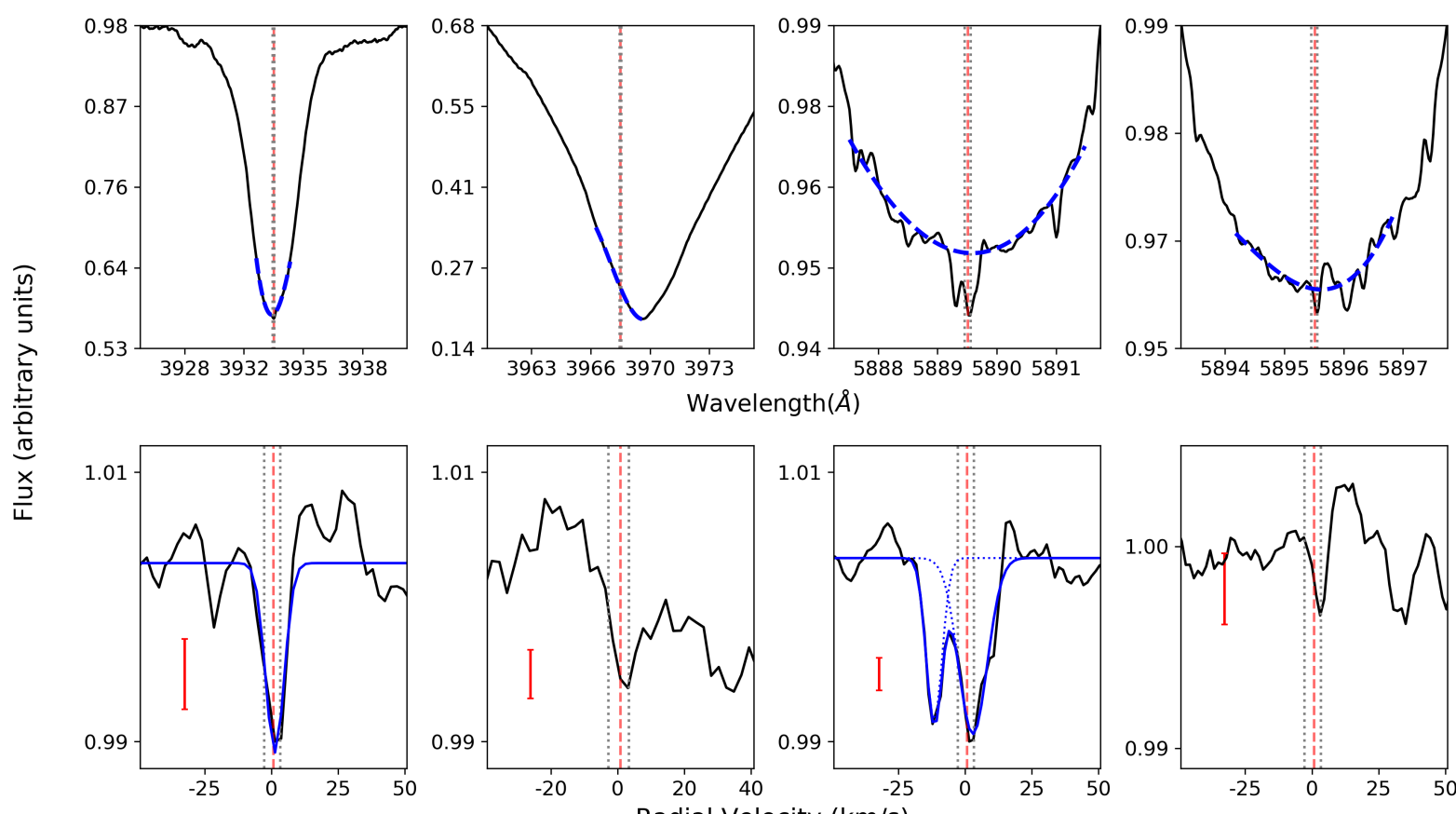

Wavelength $(\AA)$
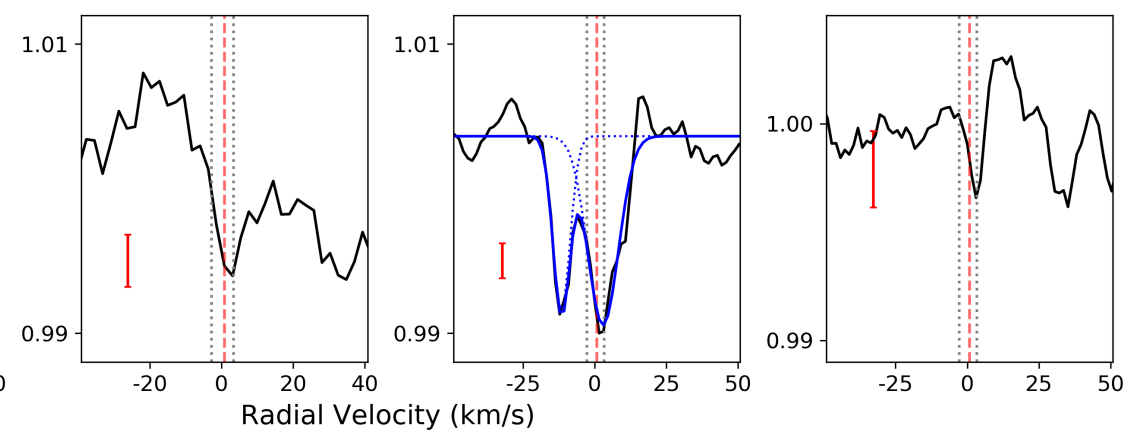

Fig. A.1. continued. 
HD 85905
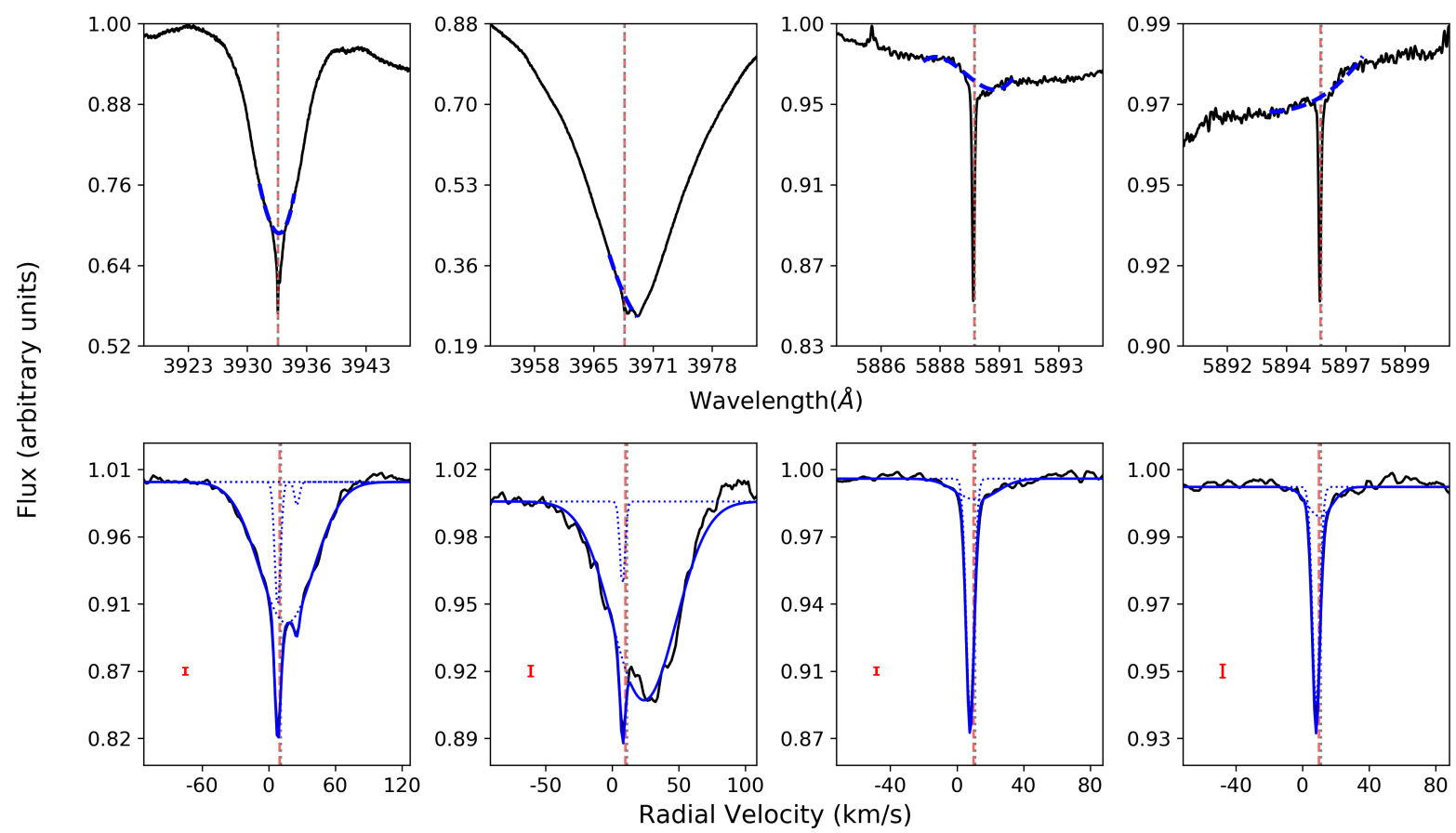

HD 98058
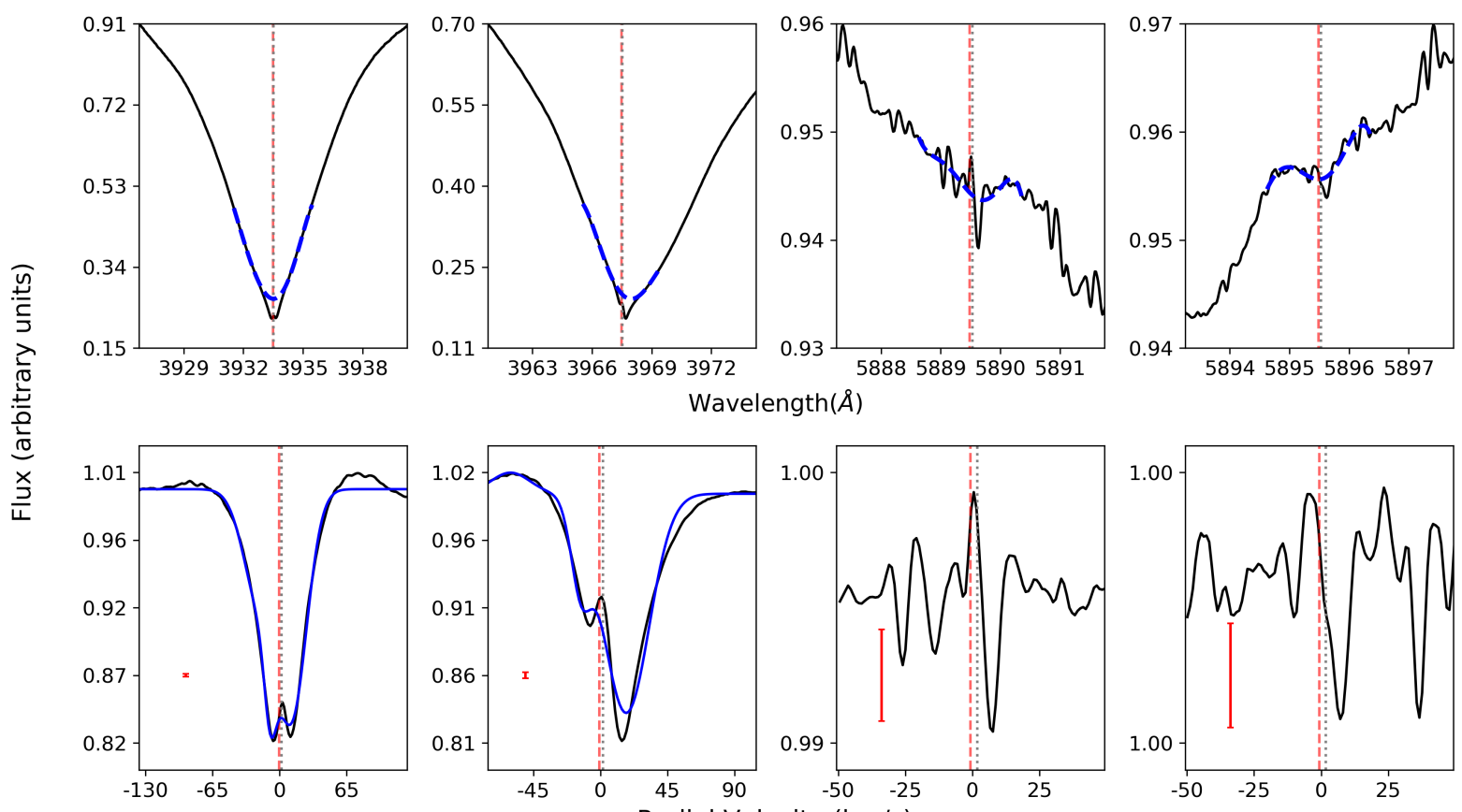
Wavelength $(\AA)$
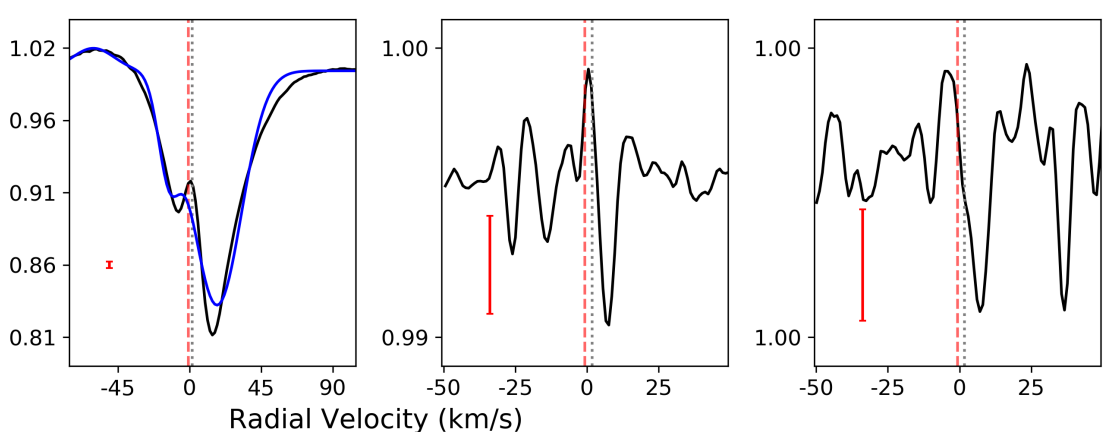

Fig. A.1. continued. 
HD 105850
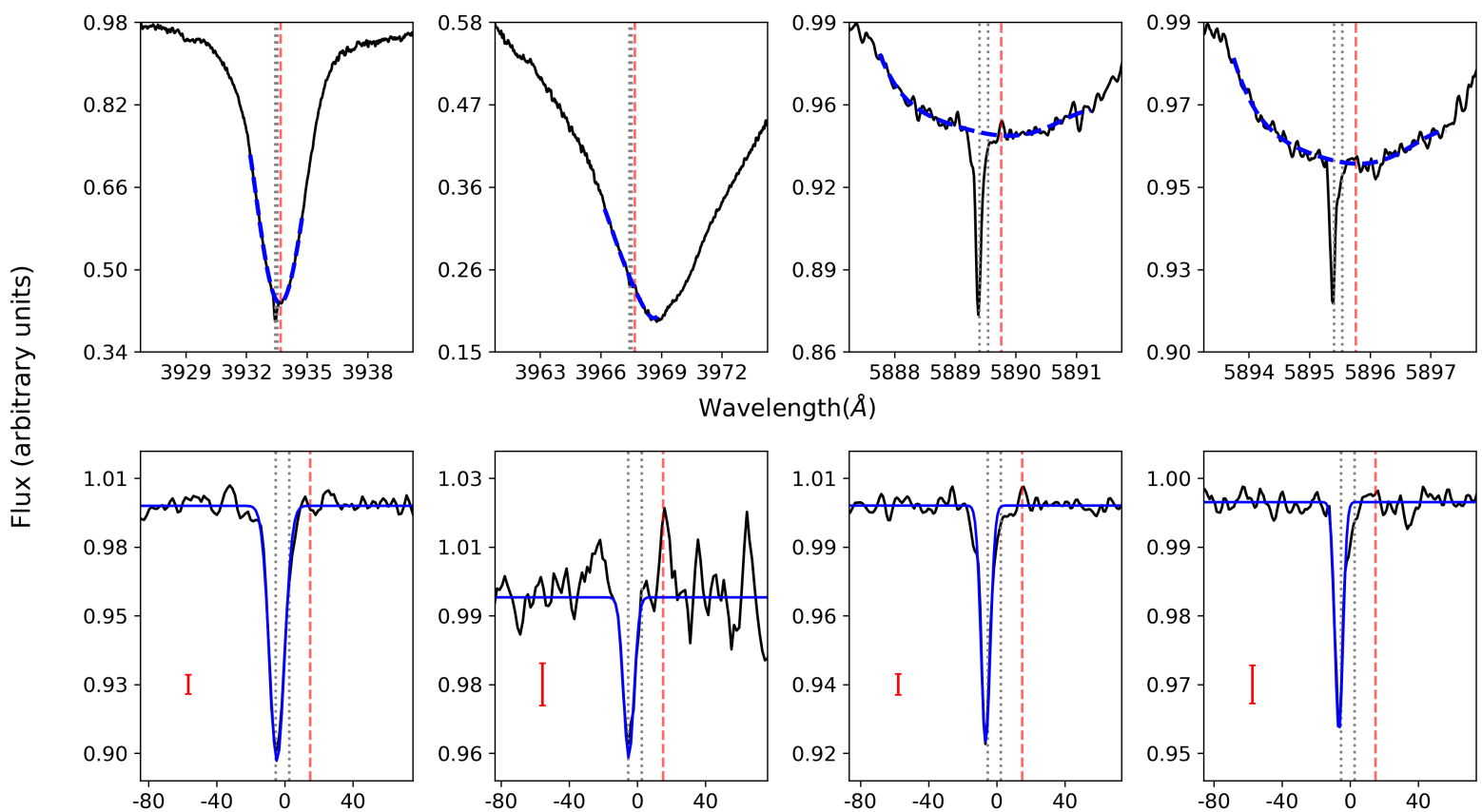
Wavelength $(\AA)$
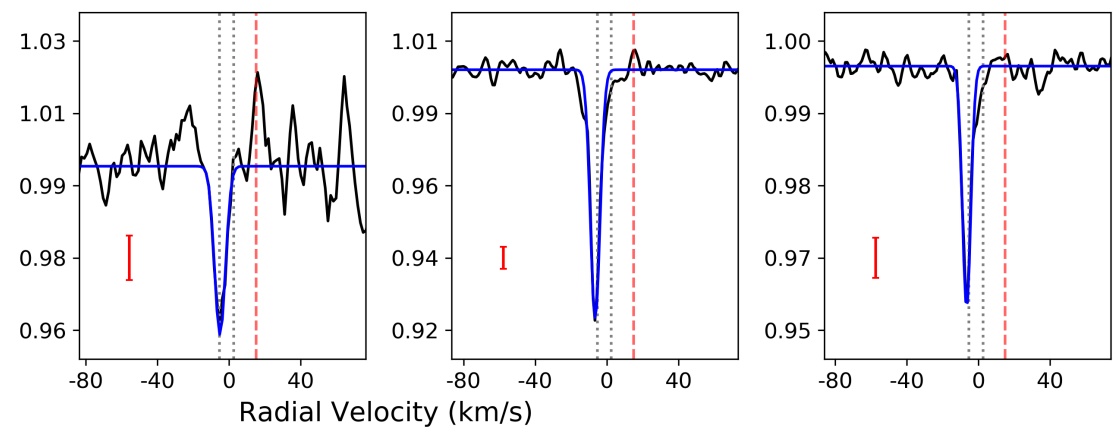

HD 108767
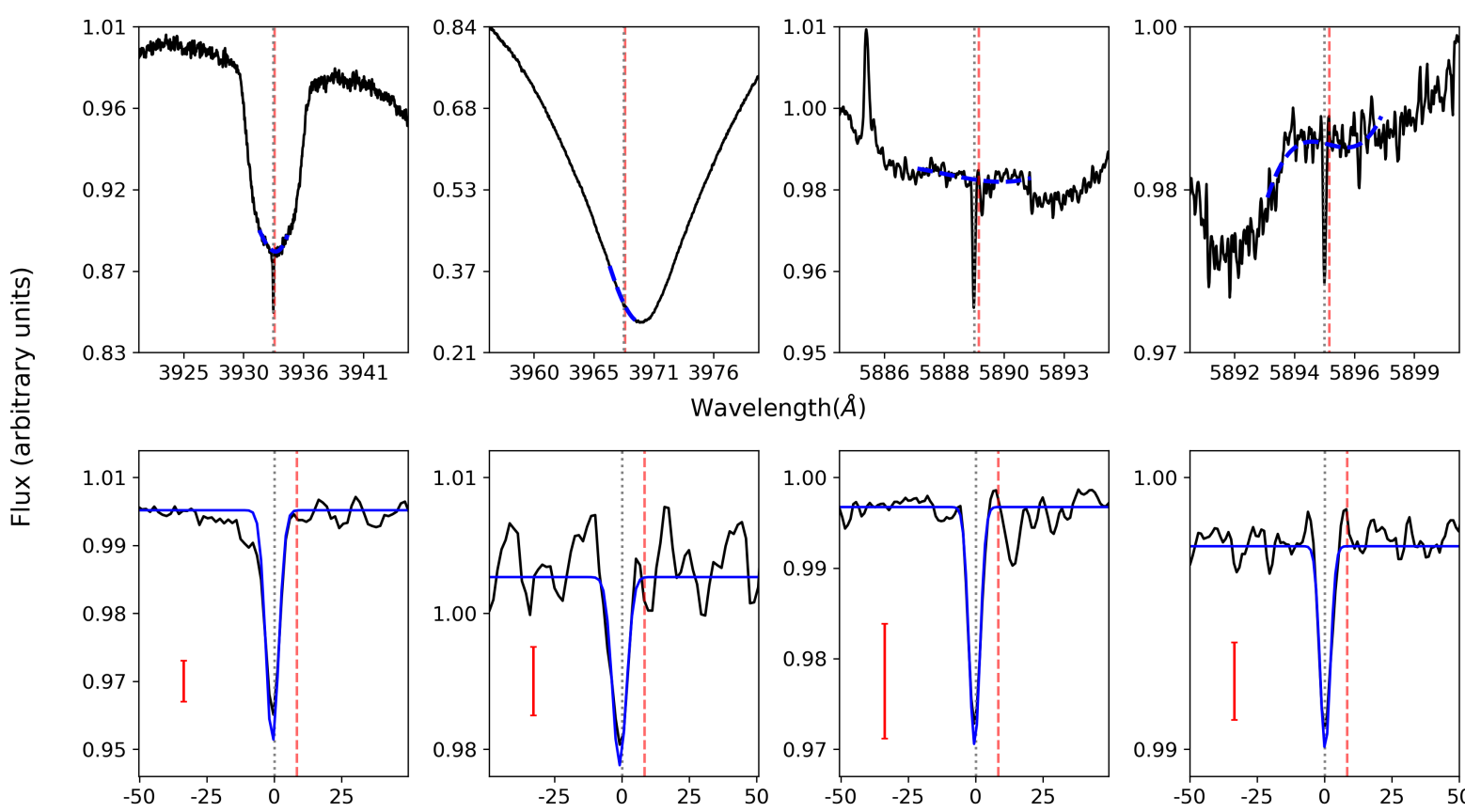
Wavelength $(\AA)$
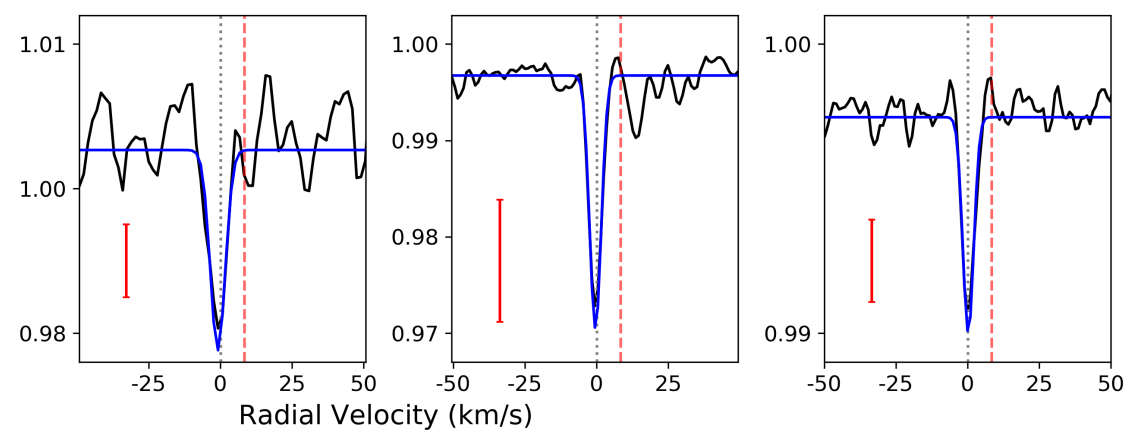

Fig. A.1. continued. 
HD 109573
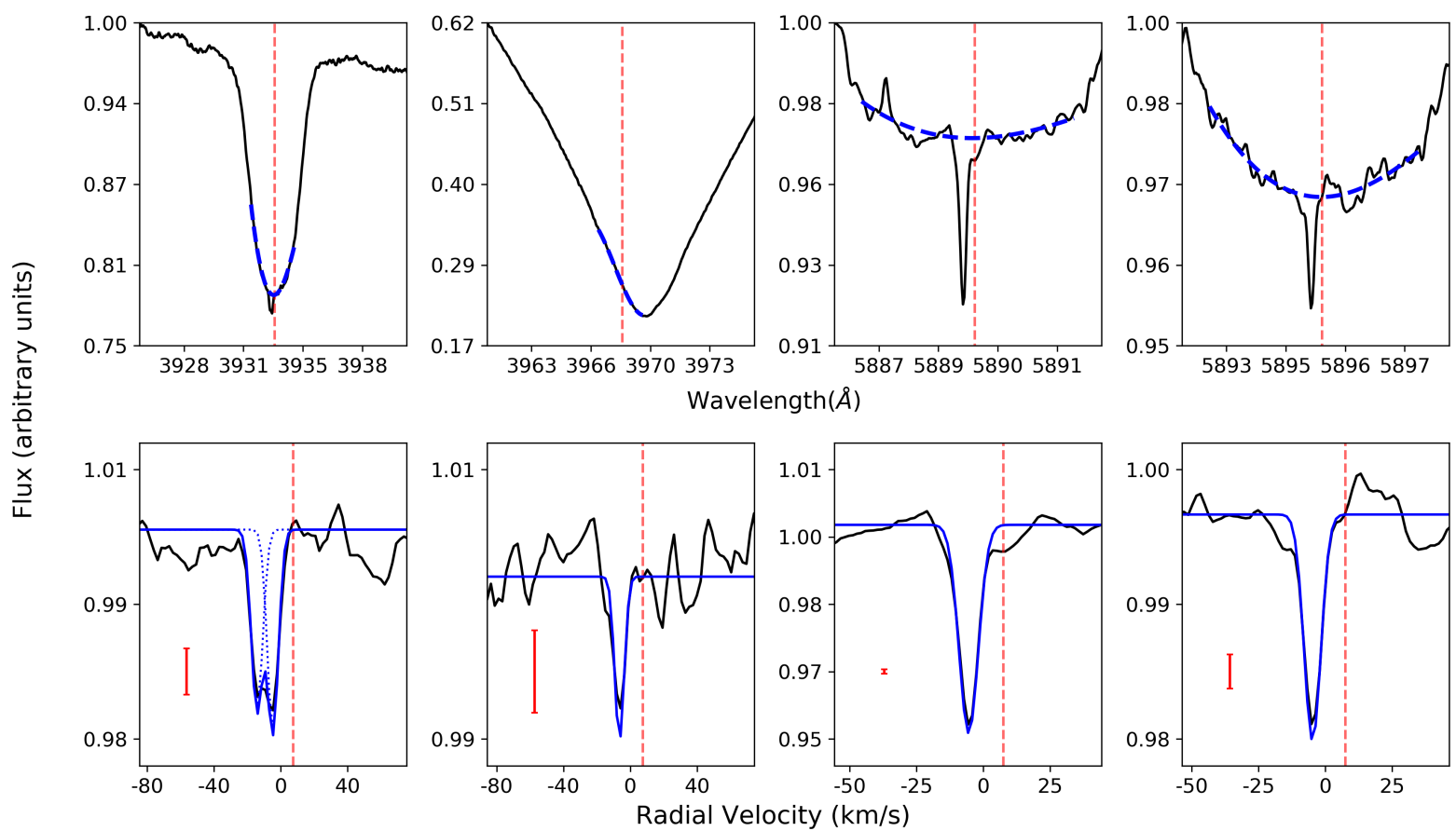

HD 110058
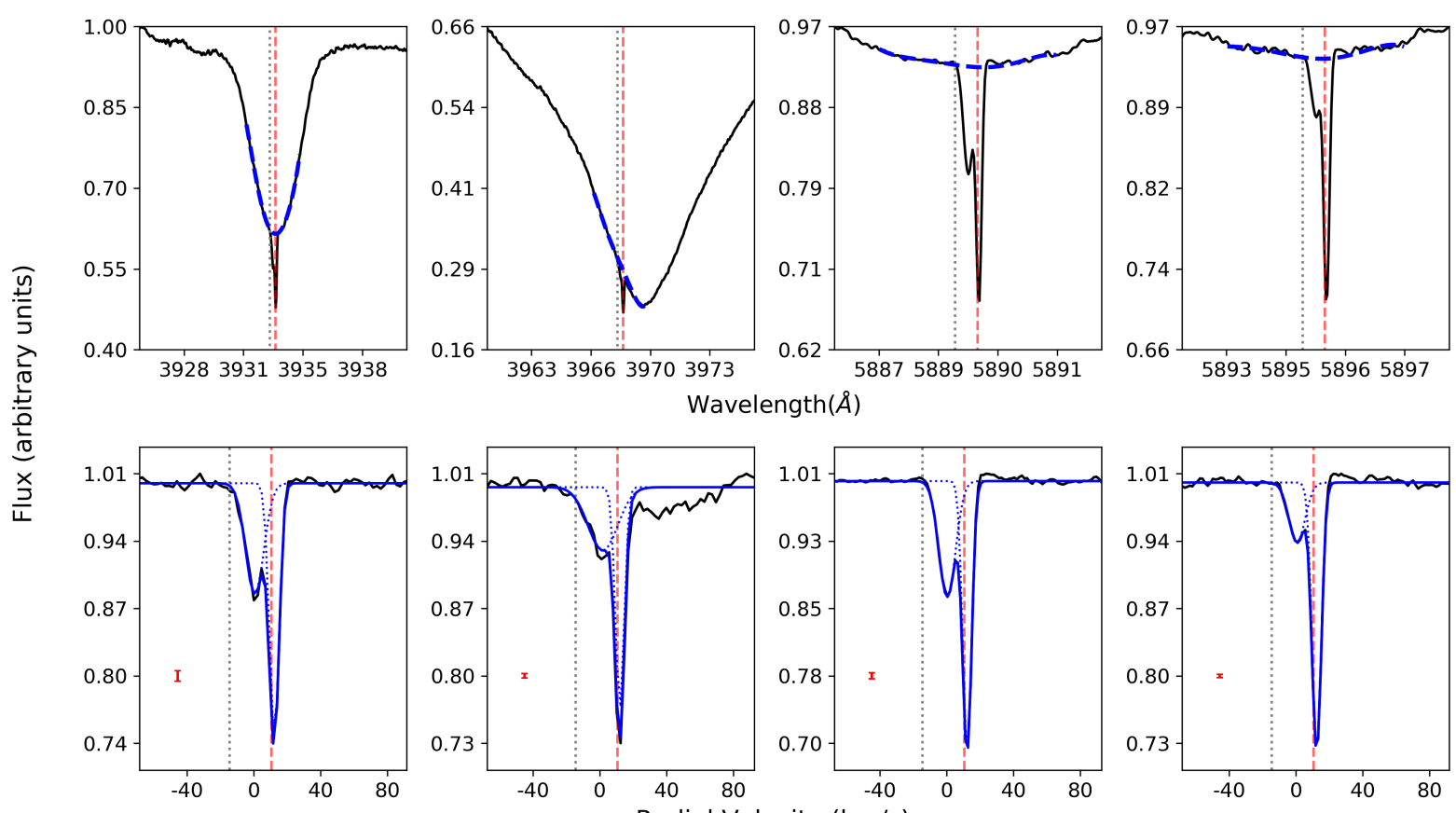

Wavelength $(\AA)$
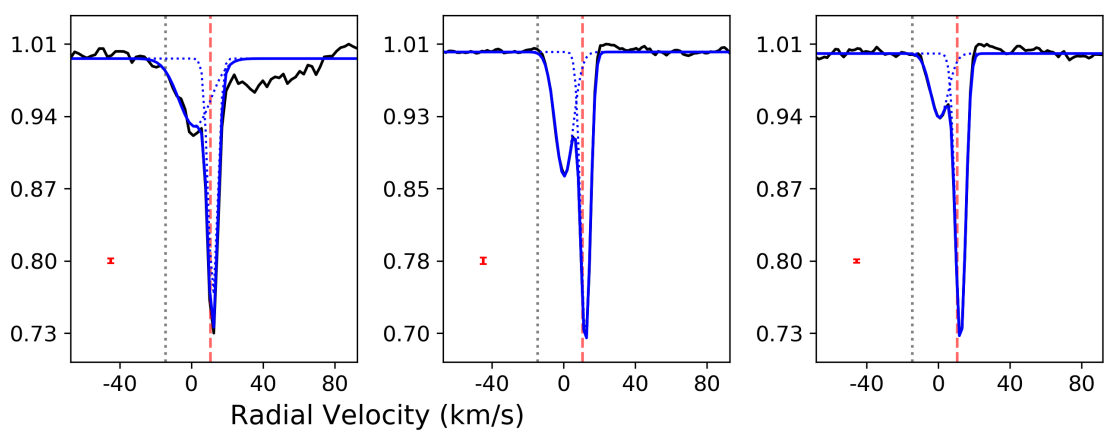

Fig. A.1. continued. 

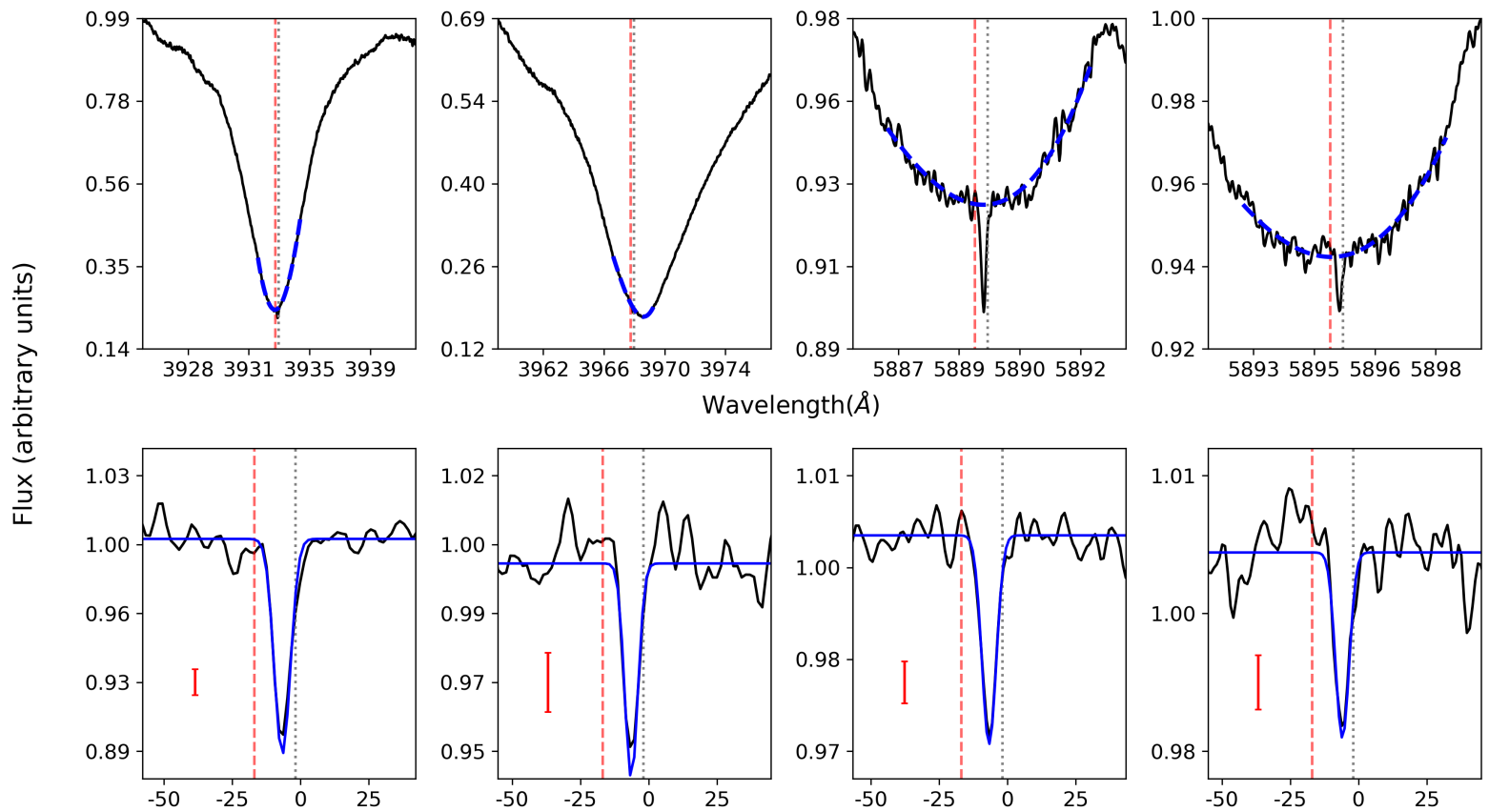
Wavelength $(\AA)$
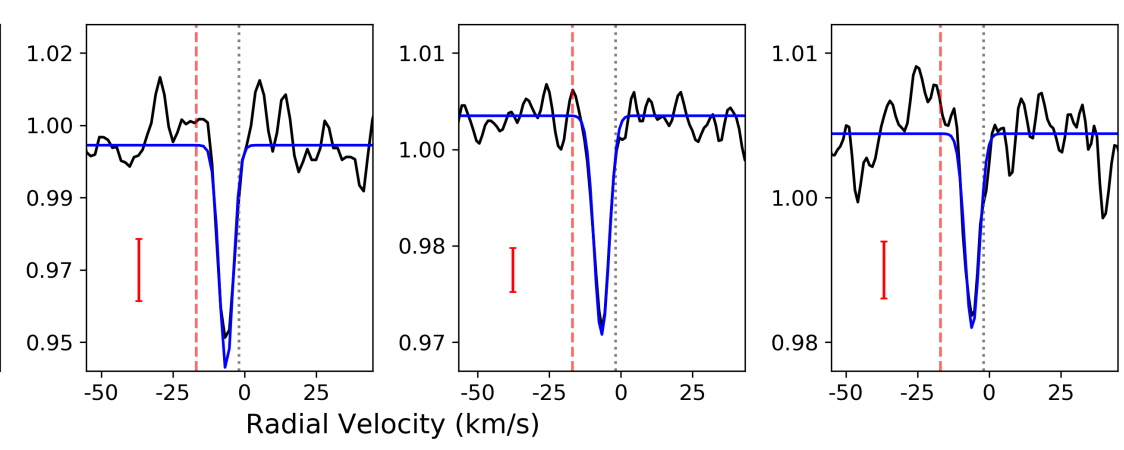

HD 125162
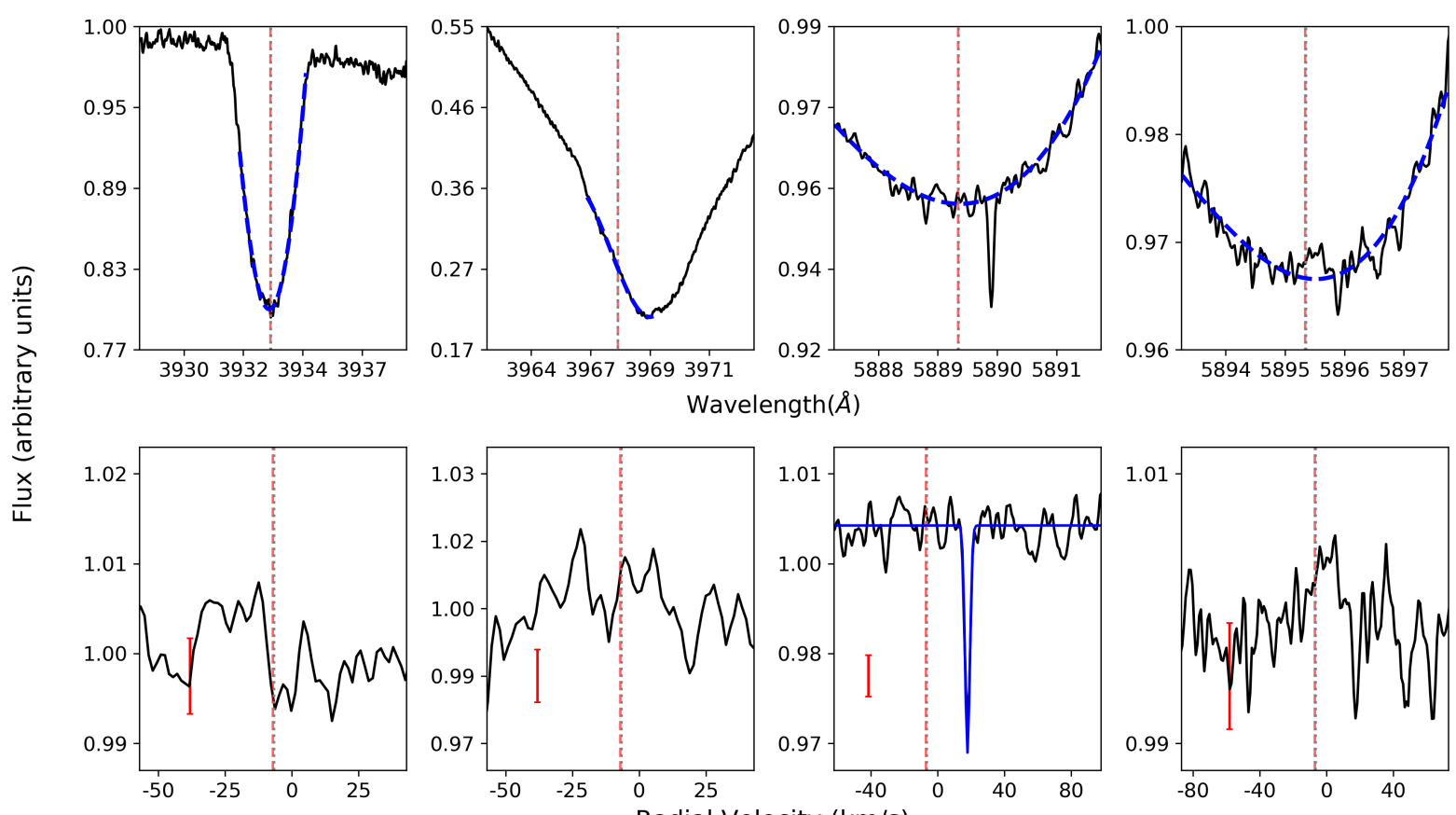

Wavelength $(\AA)$
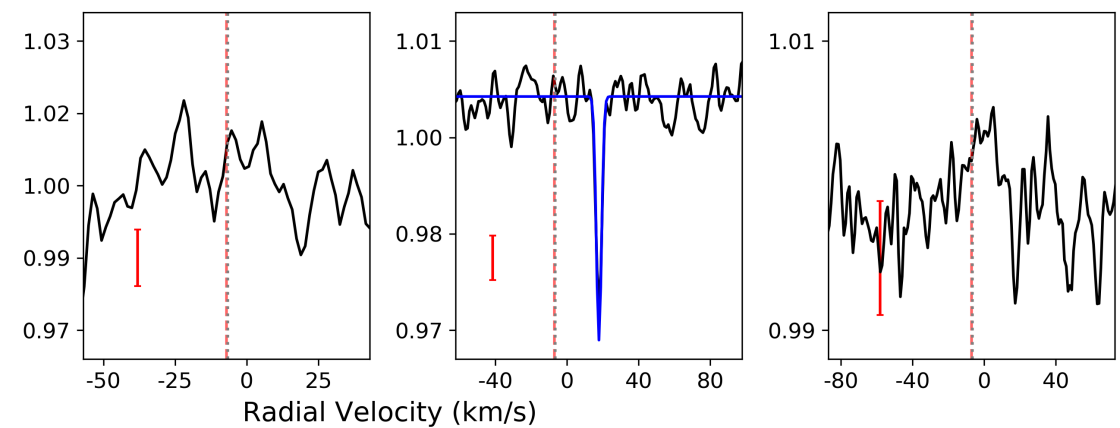

Fig. A.1. continued. 
HD 131488
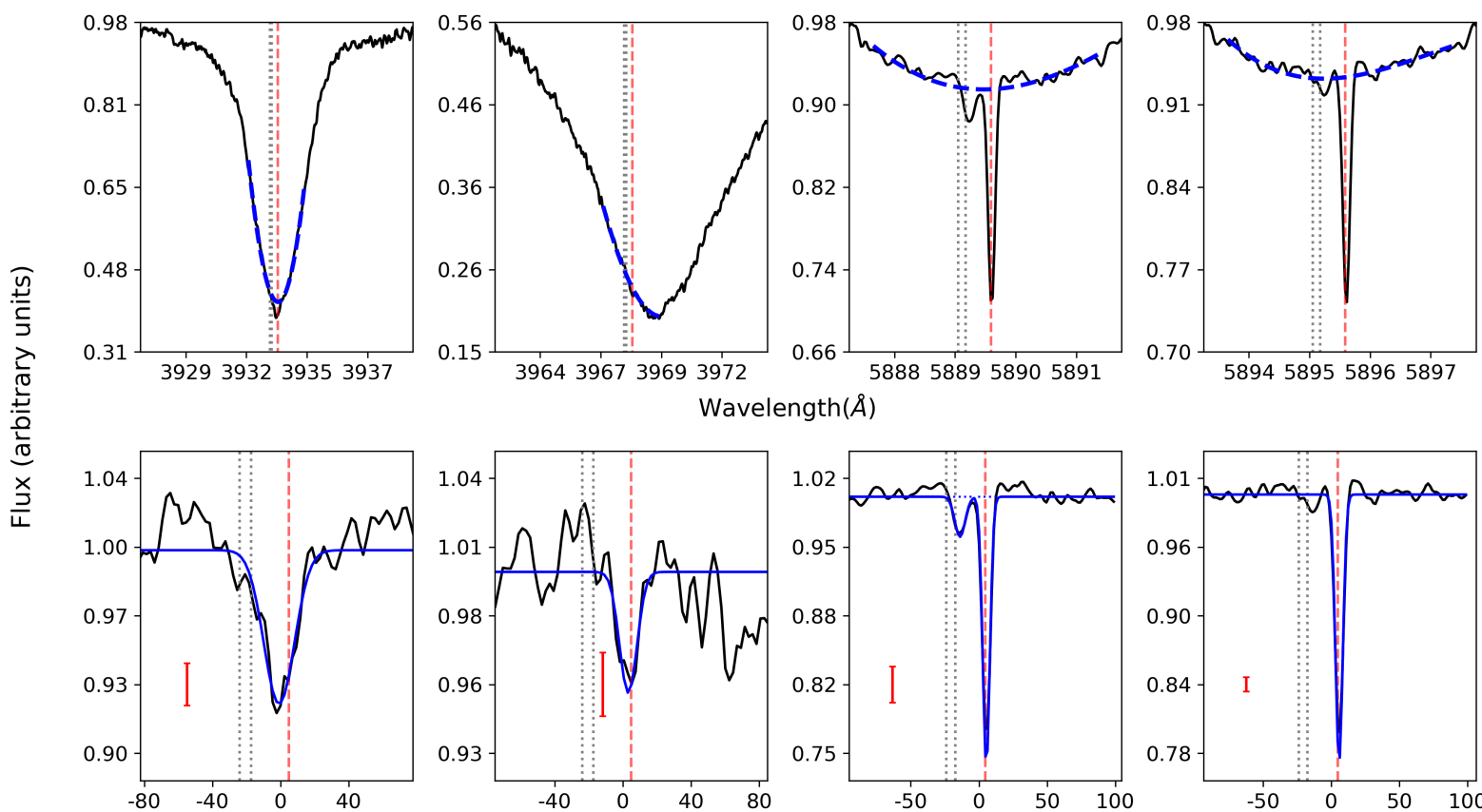

Wavelength $(\AA)$
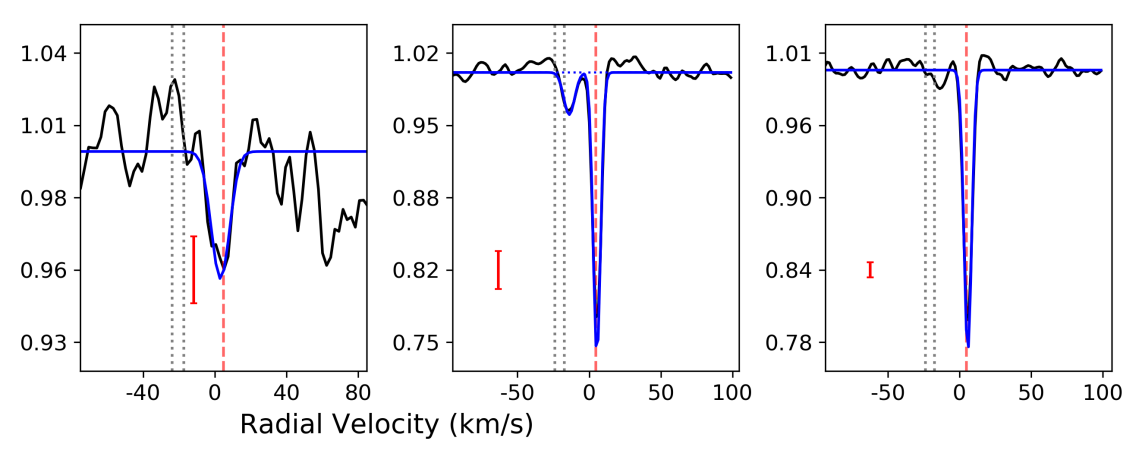

HD 131835
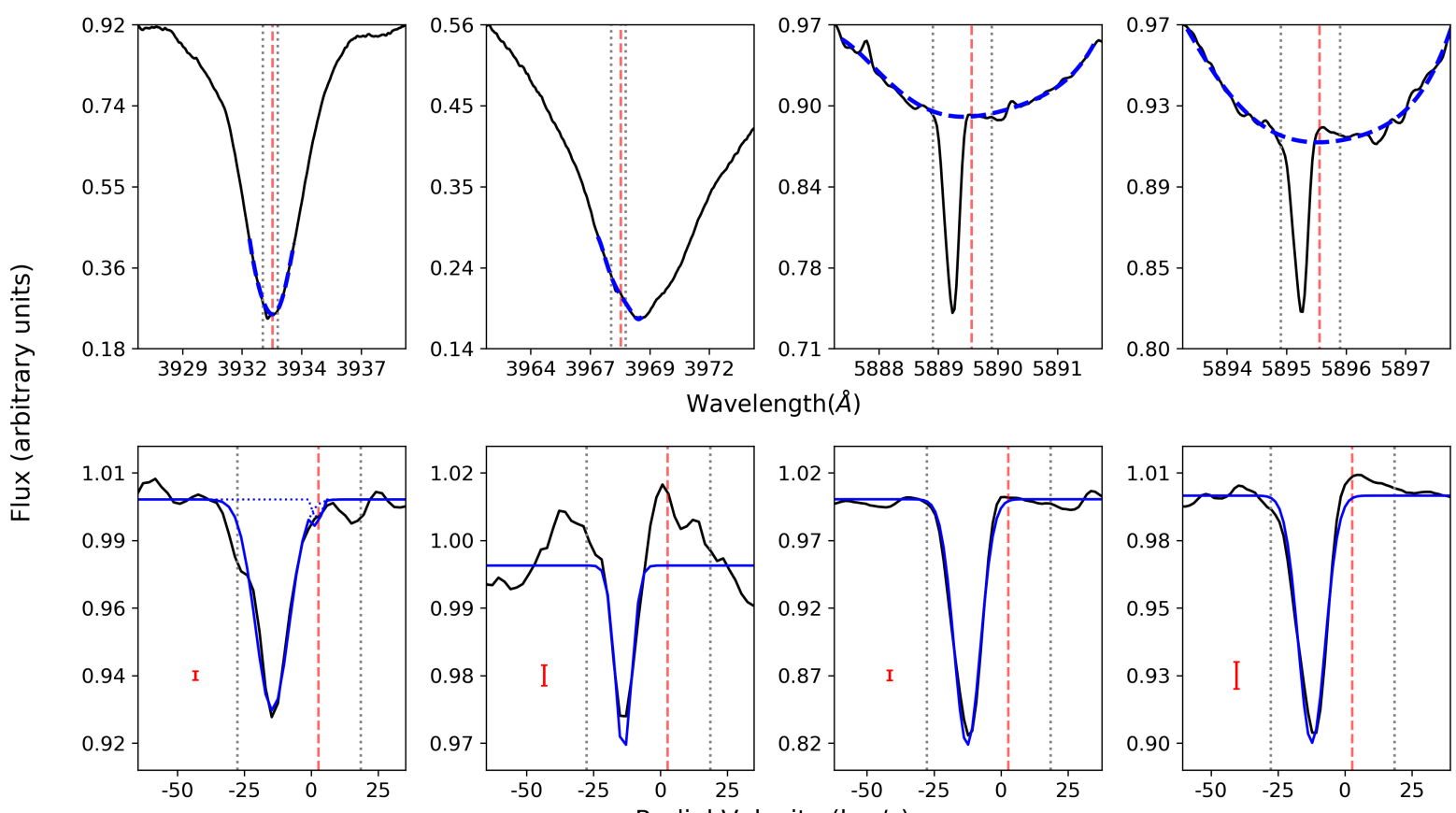

Wavelength $(\AA)$
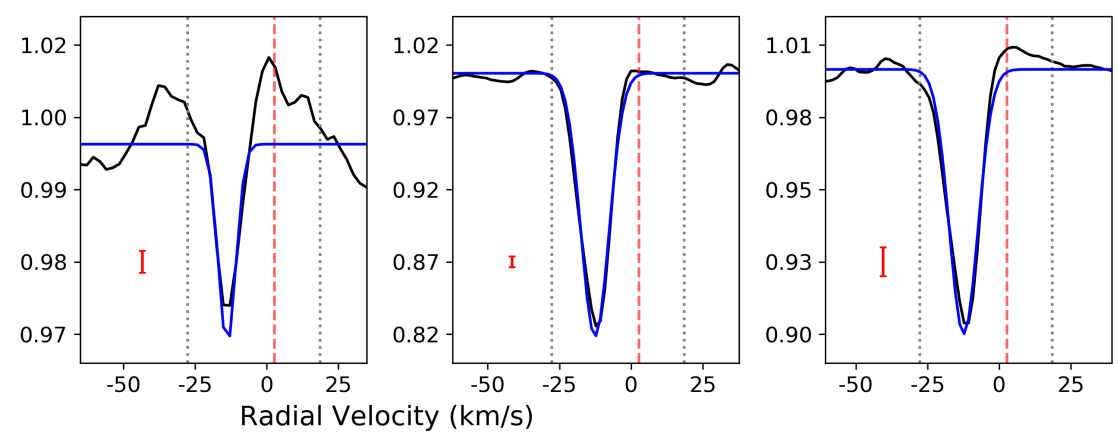

Fig. A.1. continued. 
I. Rebollido et al.: Exocomets: A spectroscopic survey

HD 138629
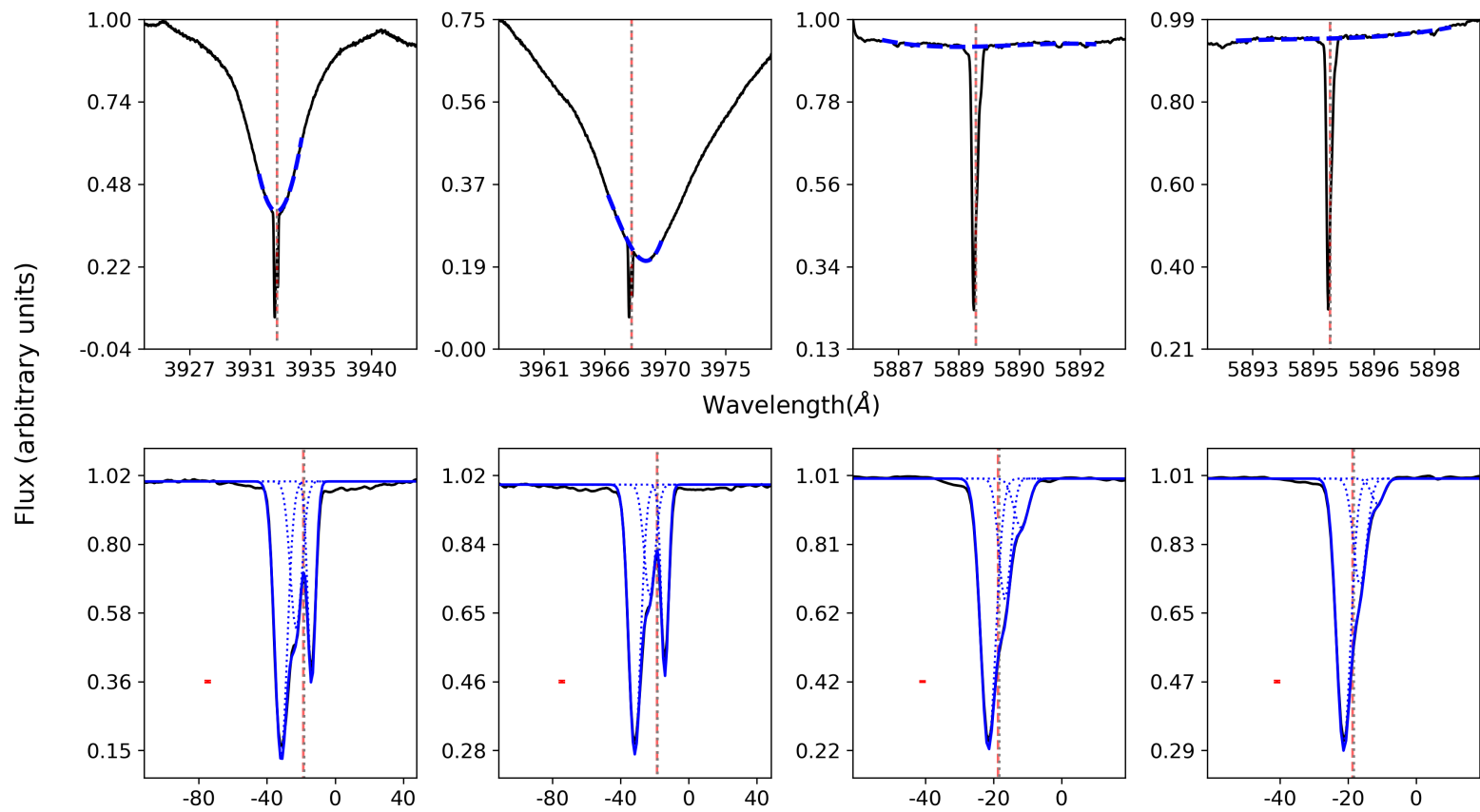

Wavelength $(\AA)$
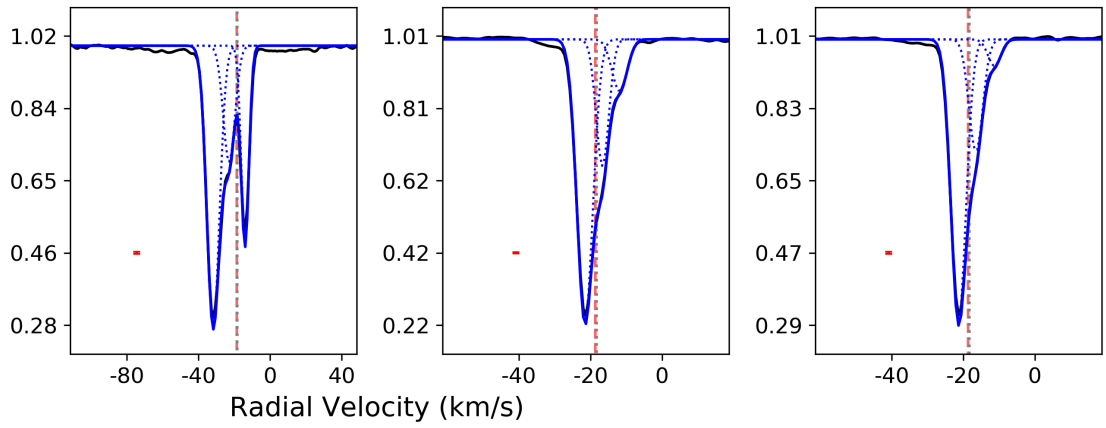

HD 138813
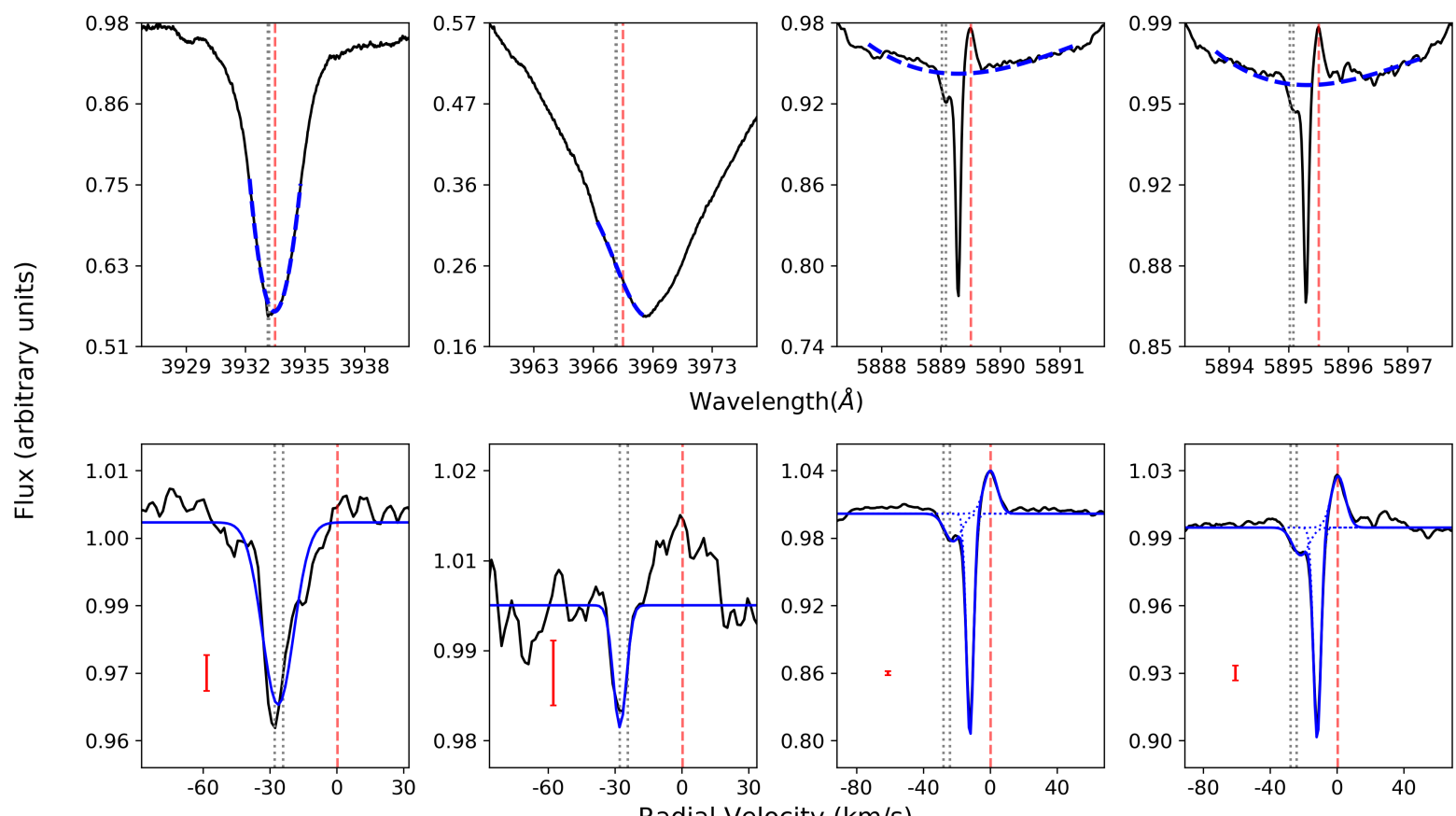

Wavelength $(\AA)$
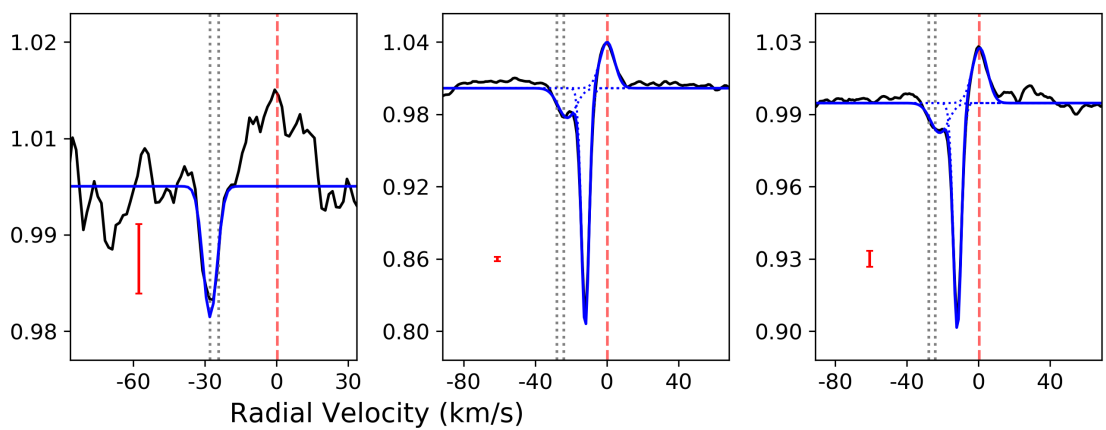

Fig. A.1. continued. 

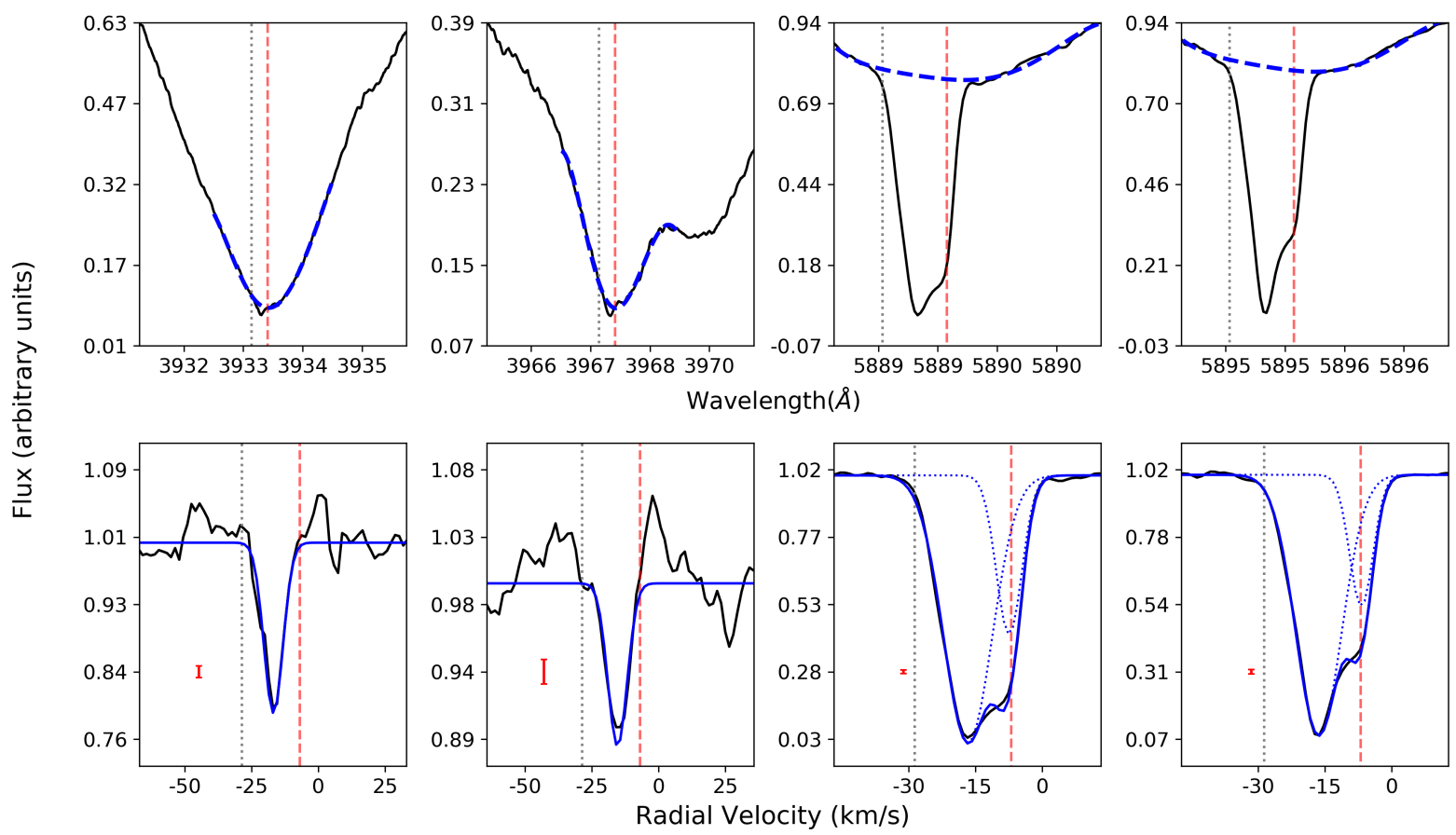

HD 142315
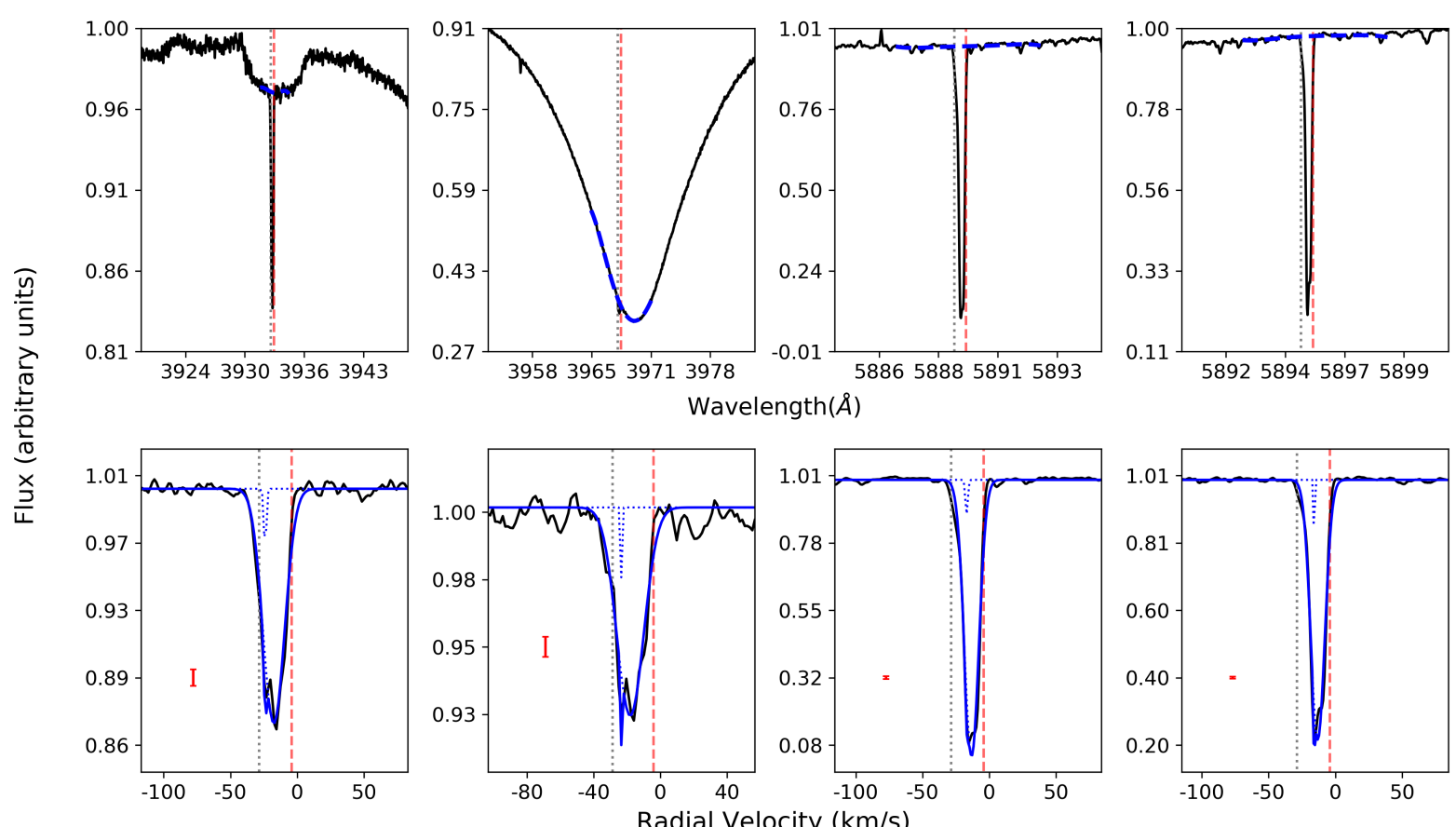

Wavelength $(\AA)$
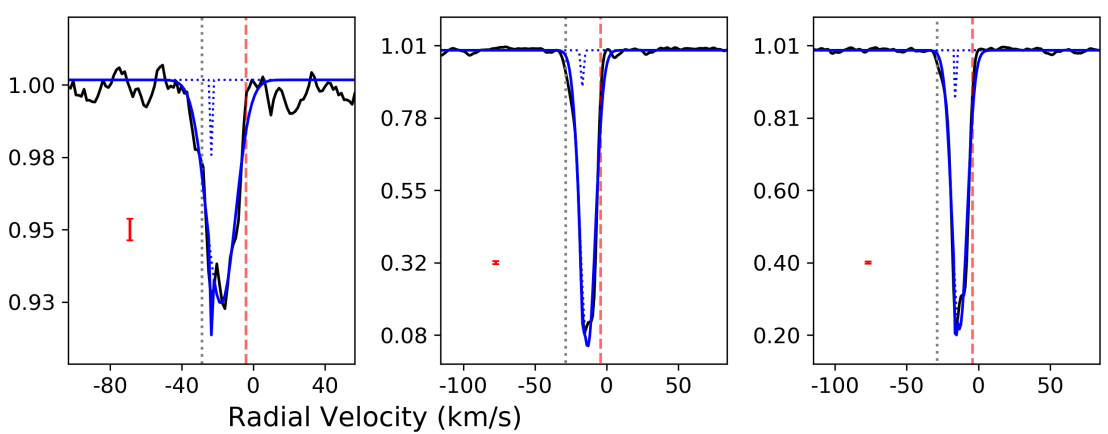

Fig. A.1. continued. 

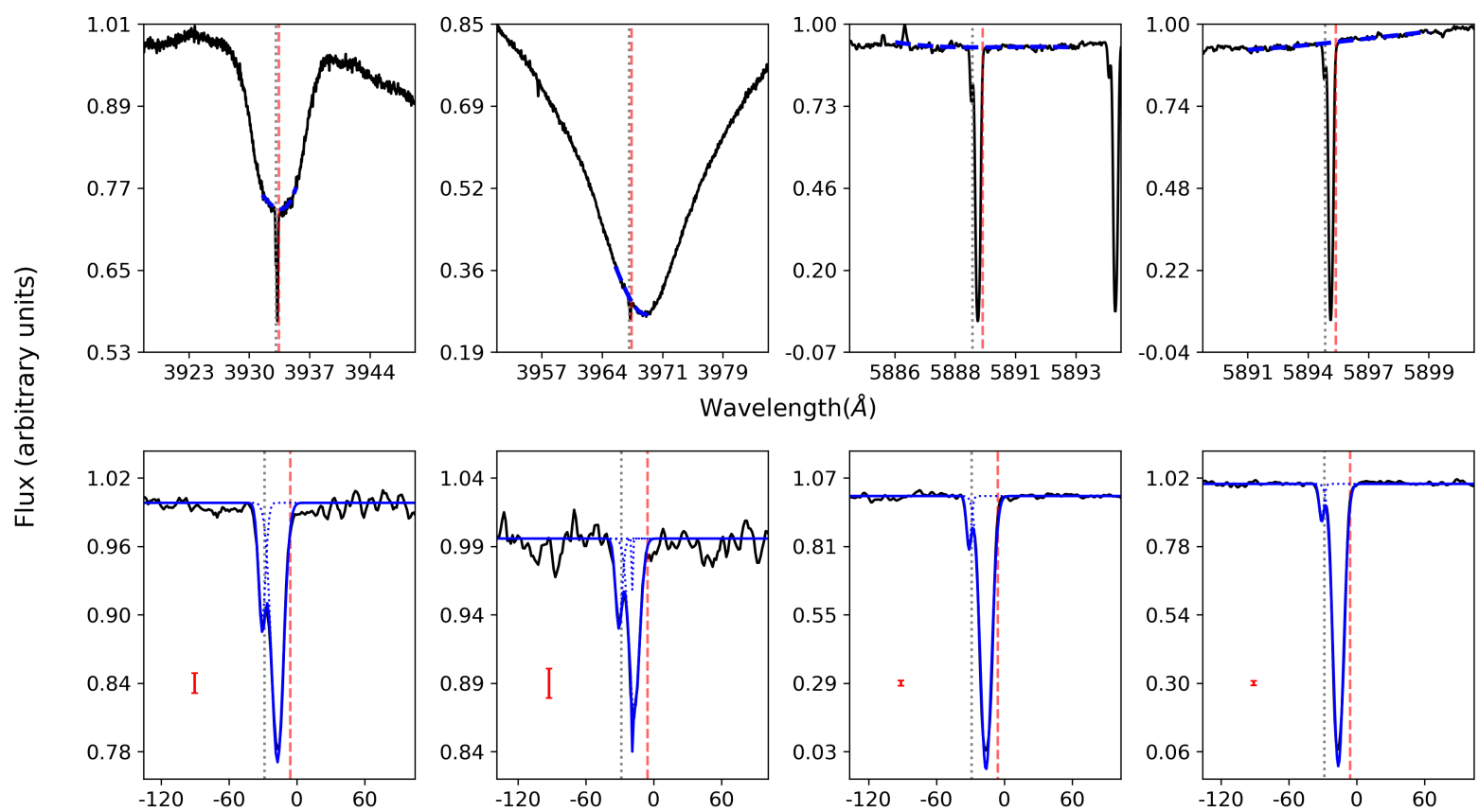
Wavelength $(\AA)$
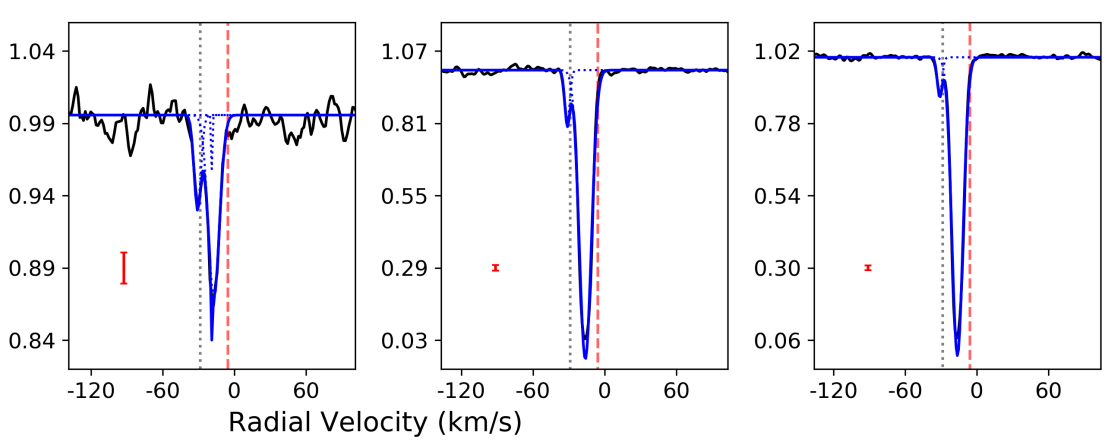

HD 144587
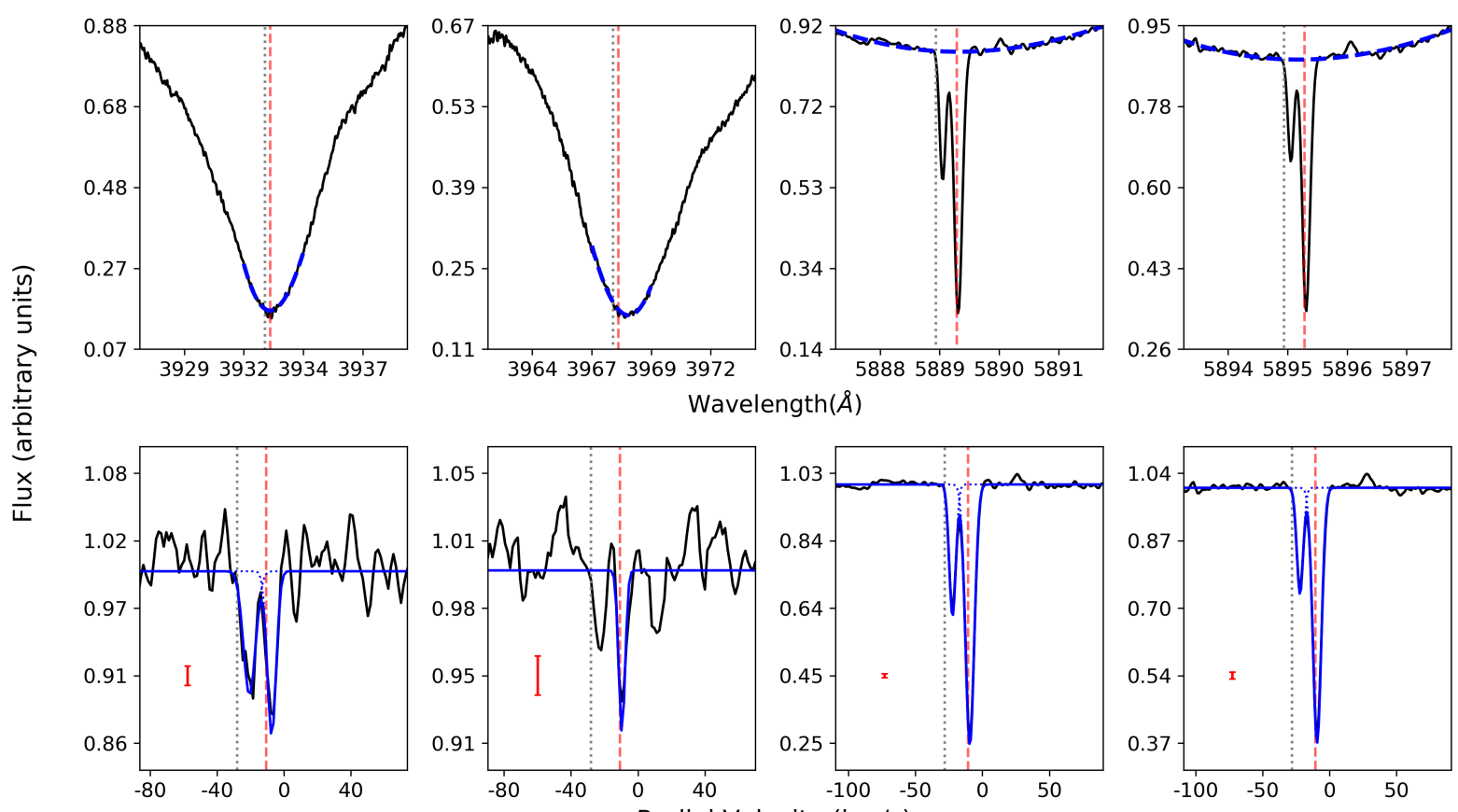
Wavelength $(\AA)$
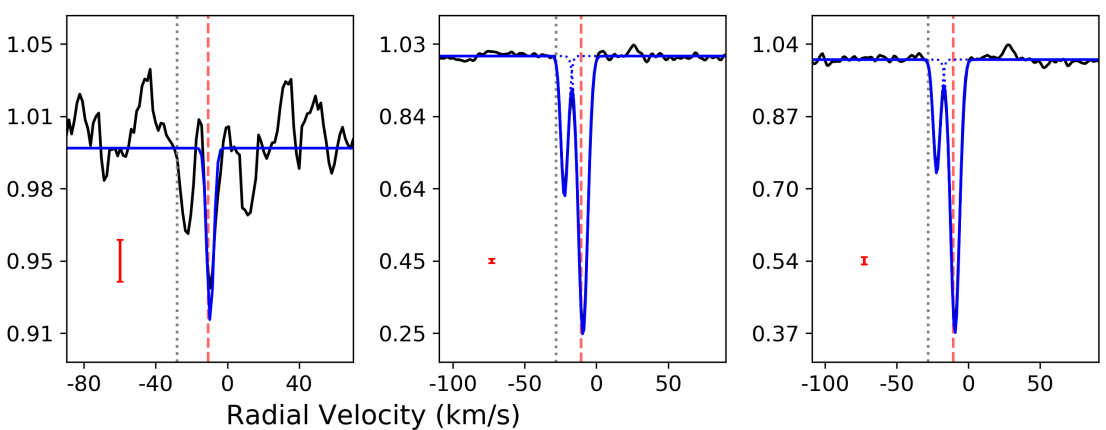

Fig. A.1. continued. 
HD 144981
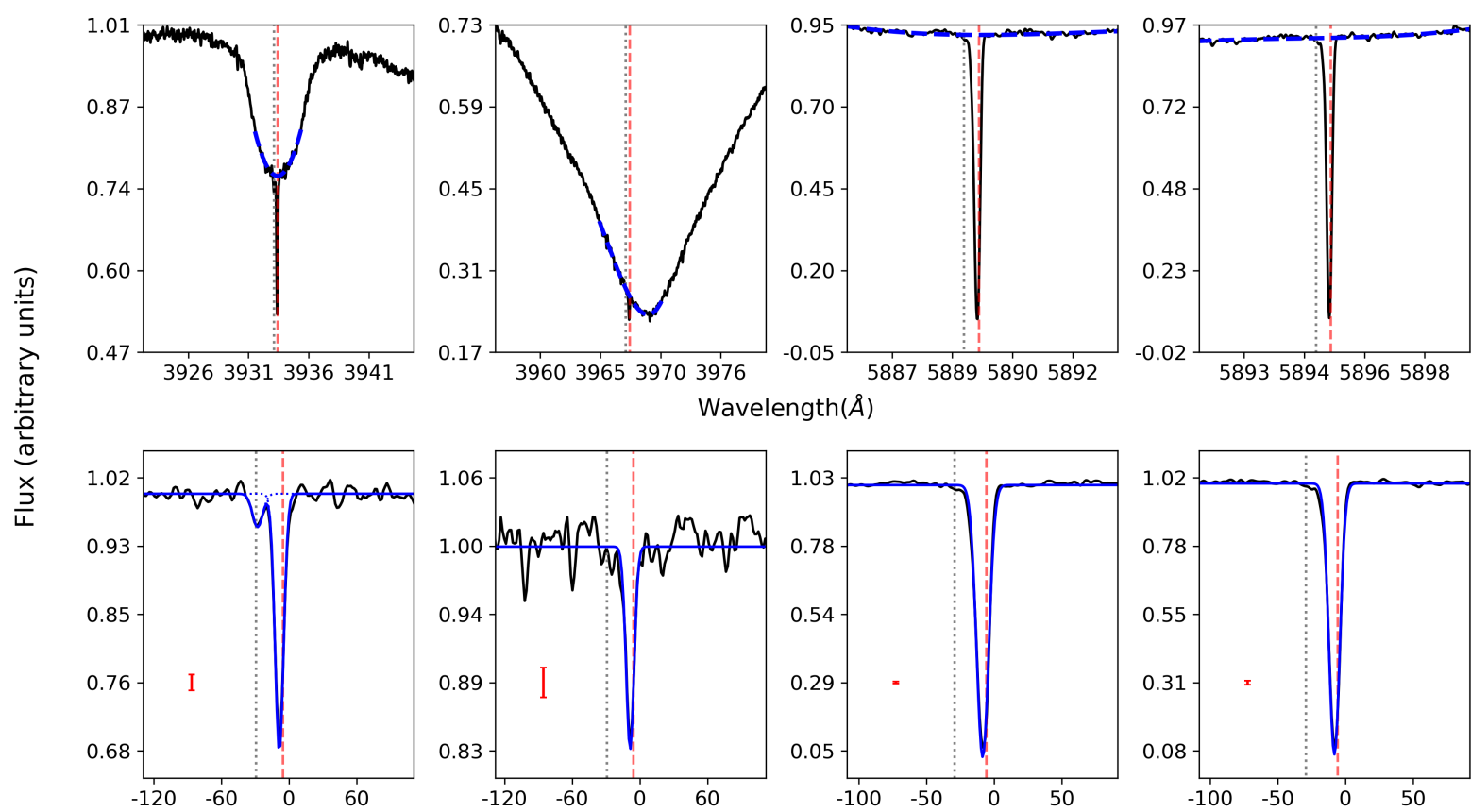
Wavelength $(\AA)$
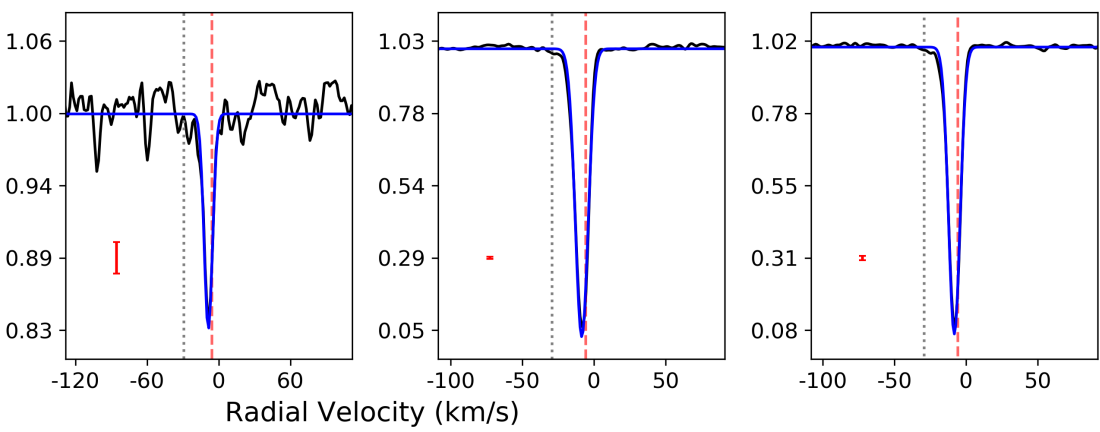

HD 145554
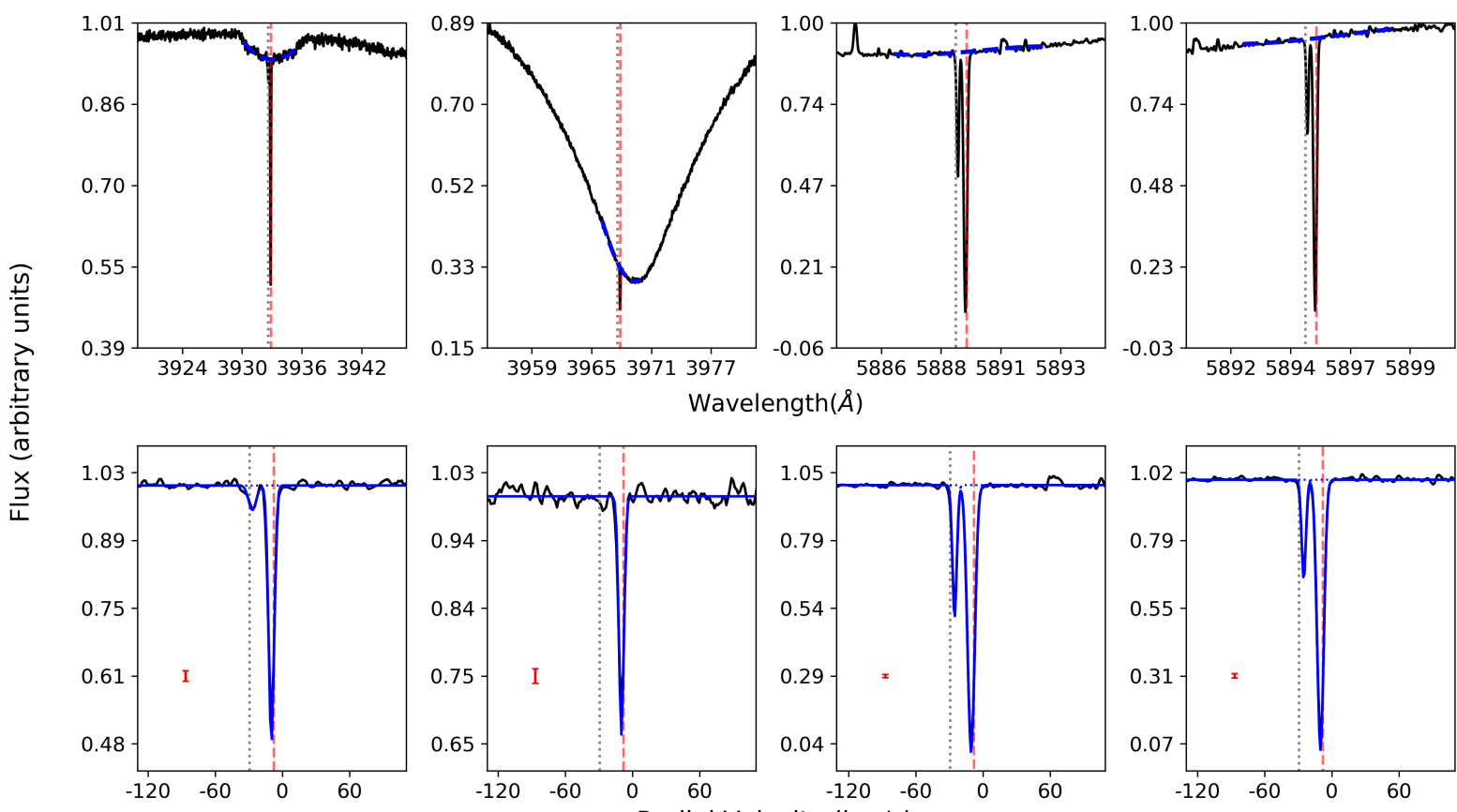
Wavelength $(\AA)$
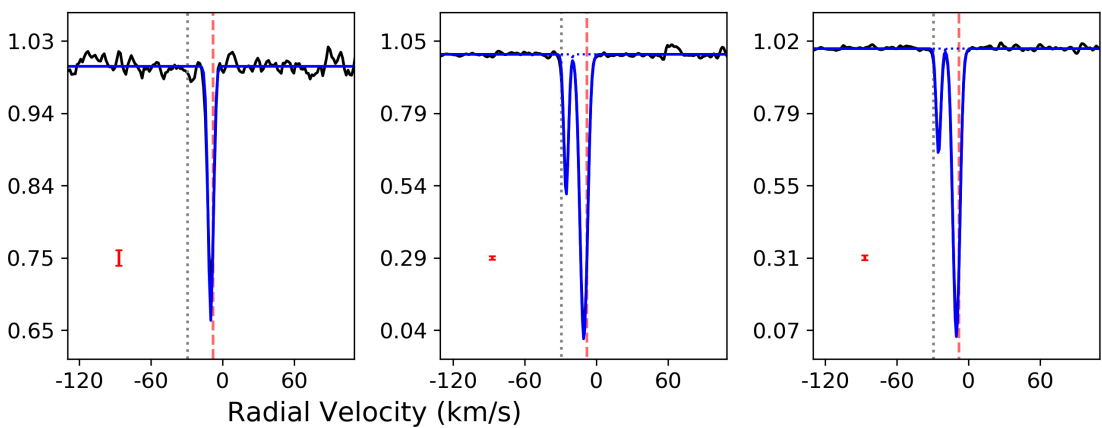

Fig. A.1. continued. 
HD 145631
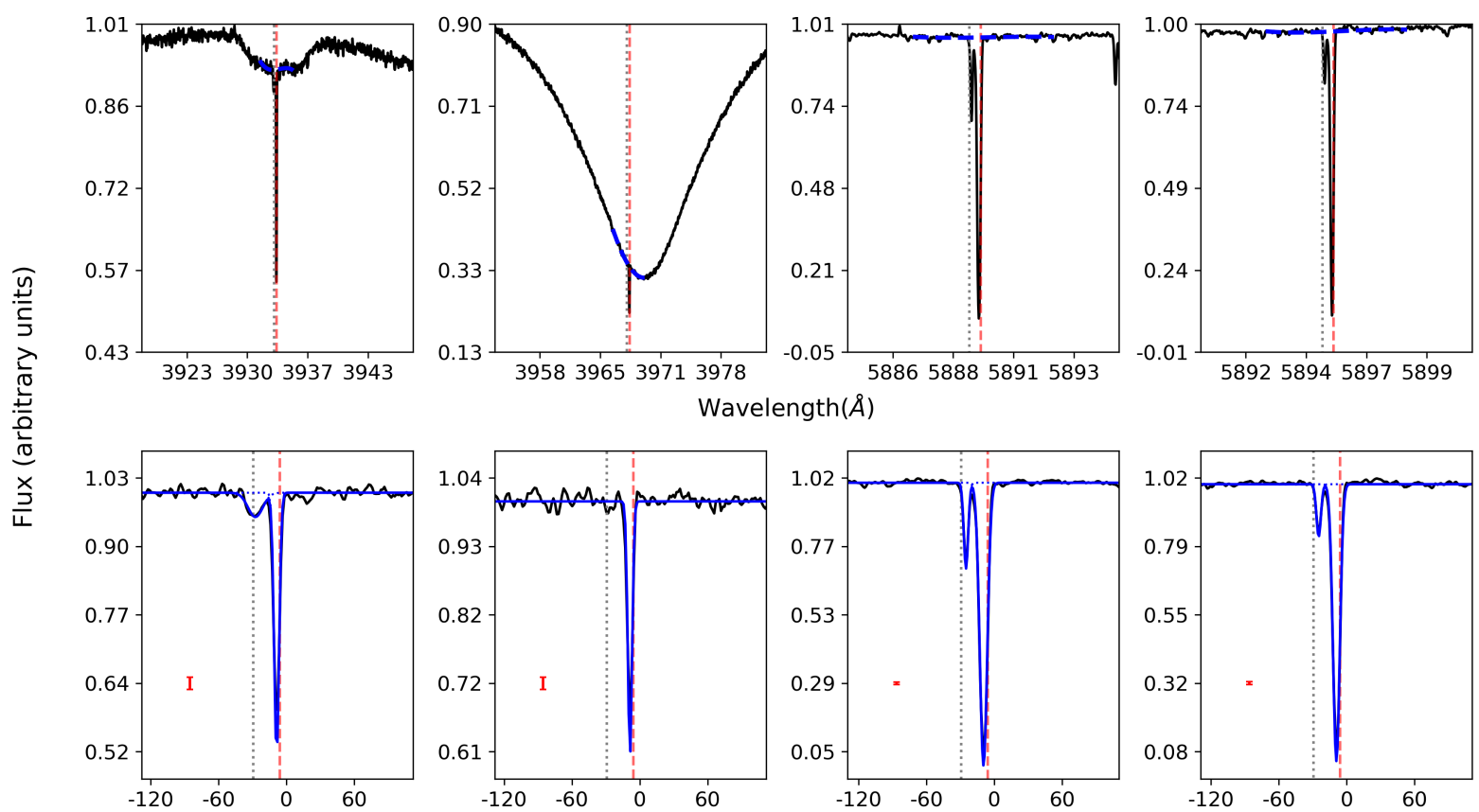
Wavelength $(\AA)$
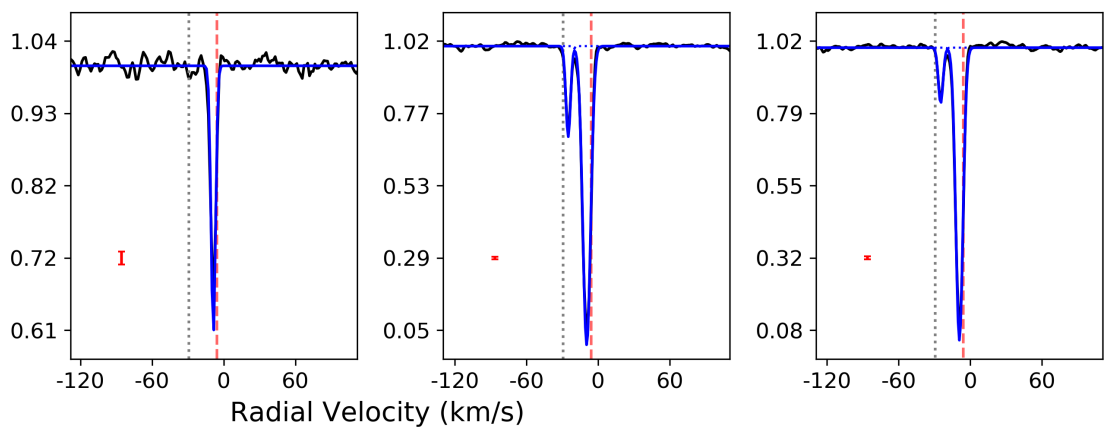

HD 145964
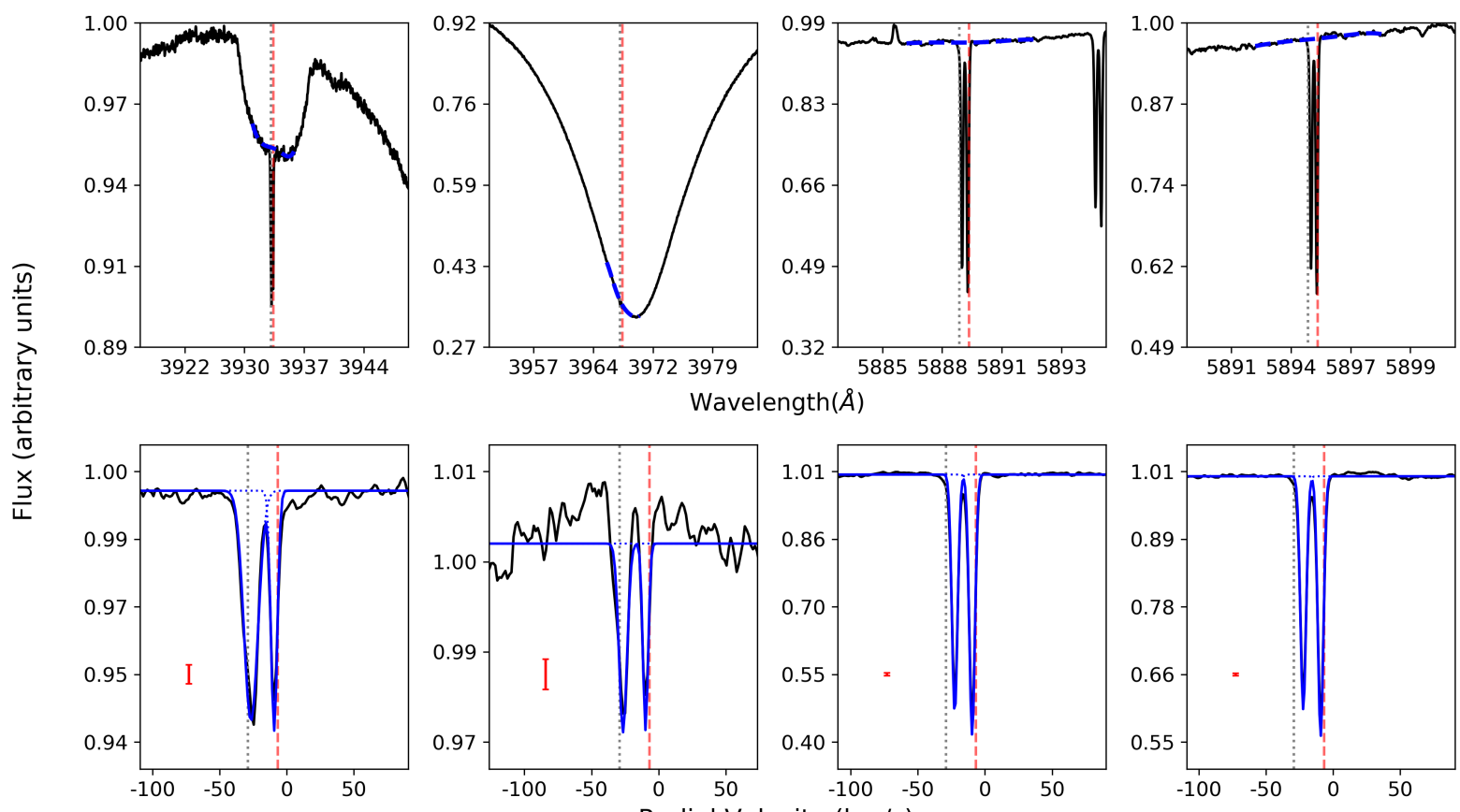

Wavelength $(\AA)$
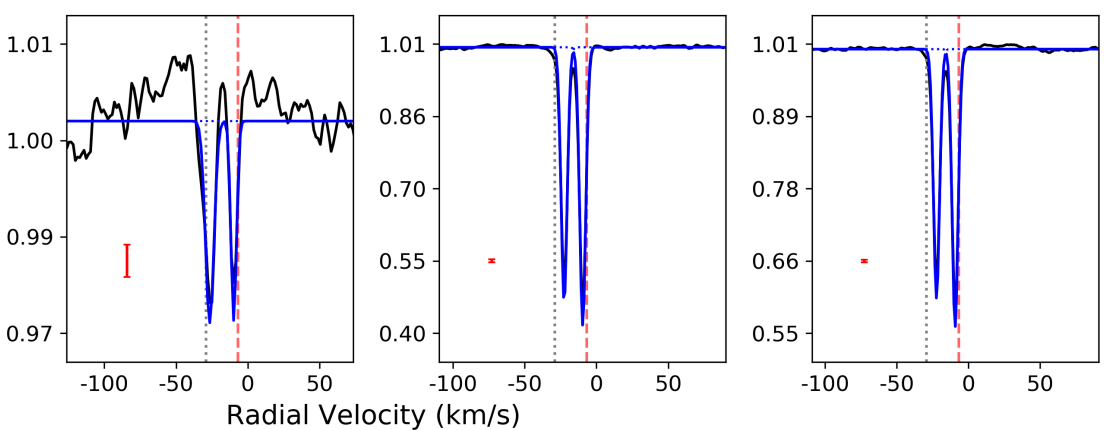

Fig. A.1. continued. 

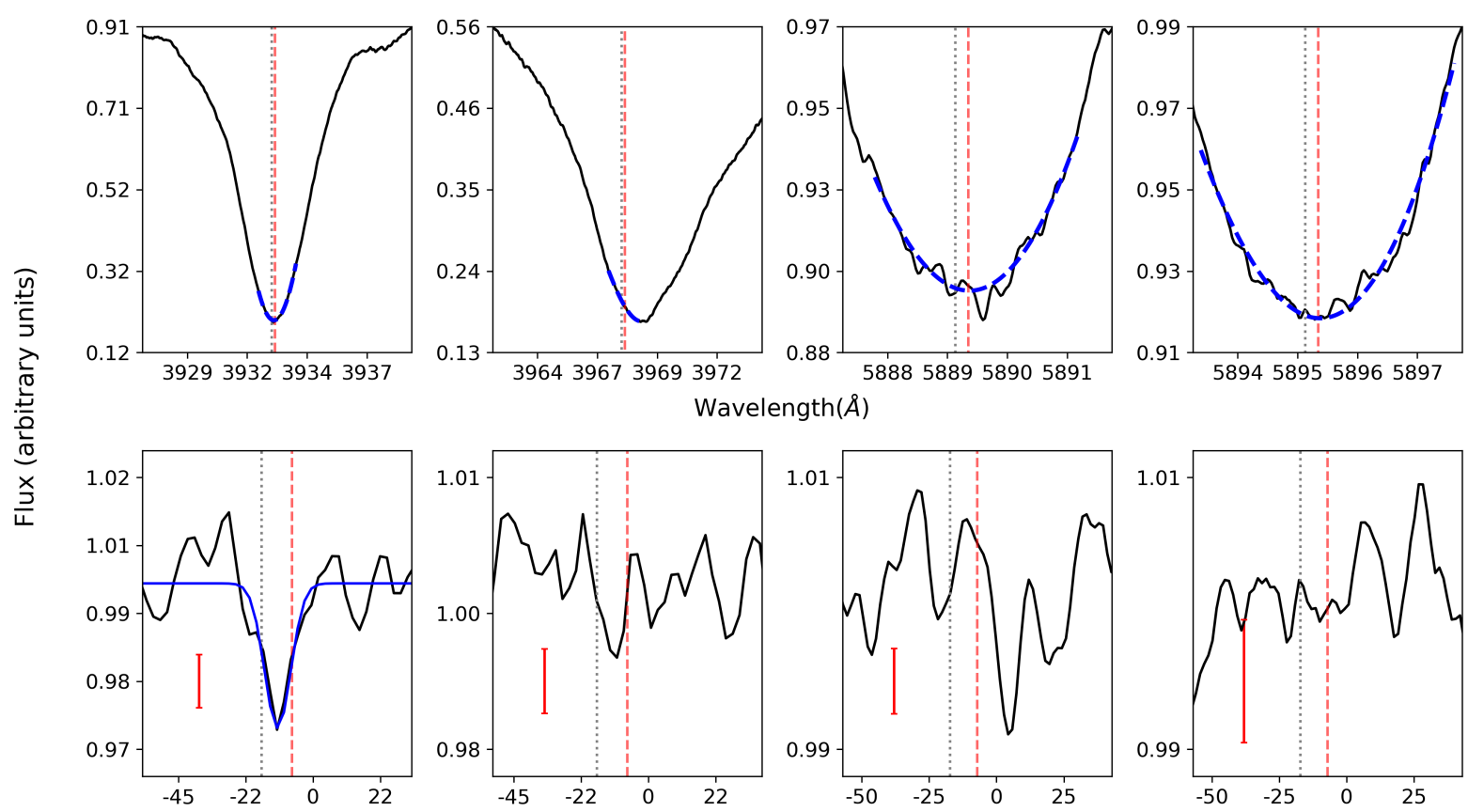

Wavelength $(\AA)$
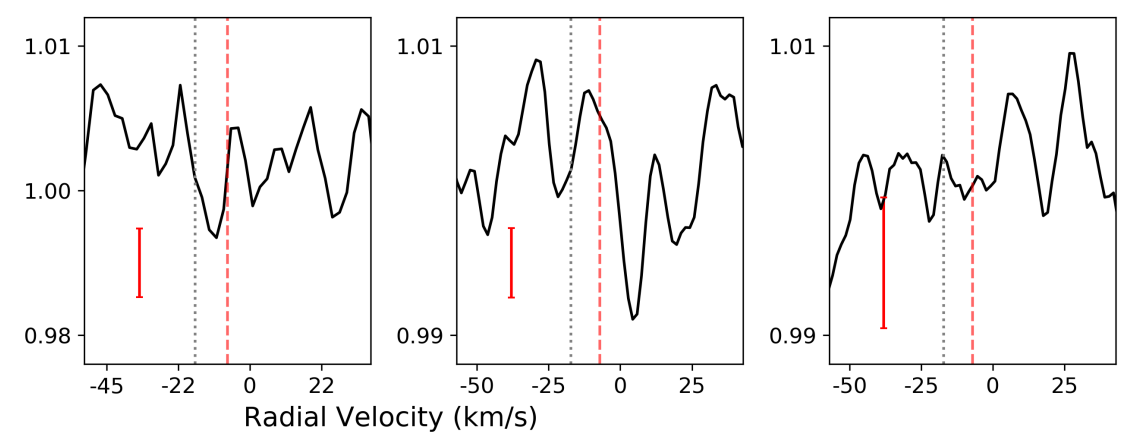

HD 146606
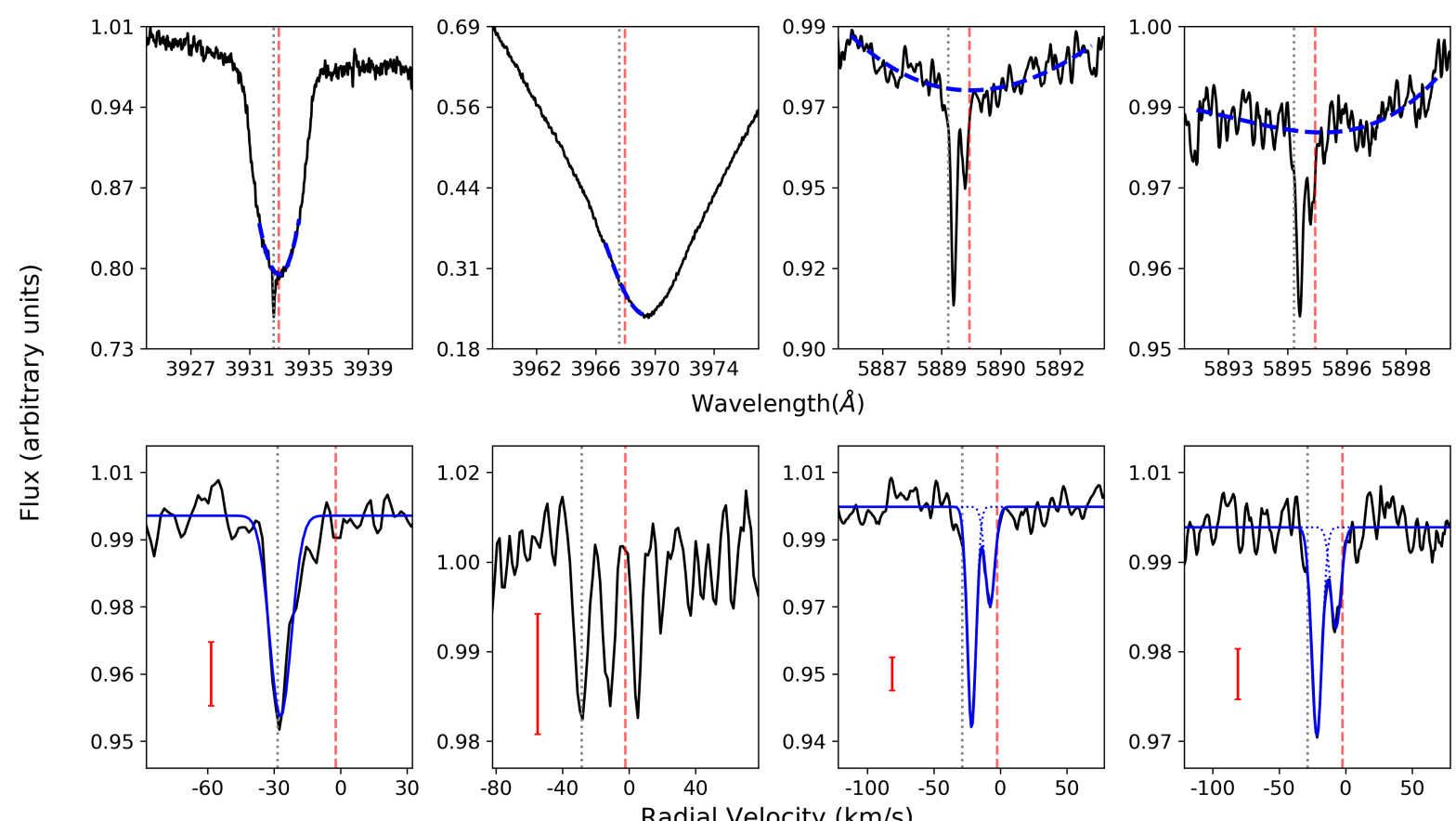
Wavelength $(\AA)$
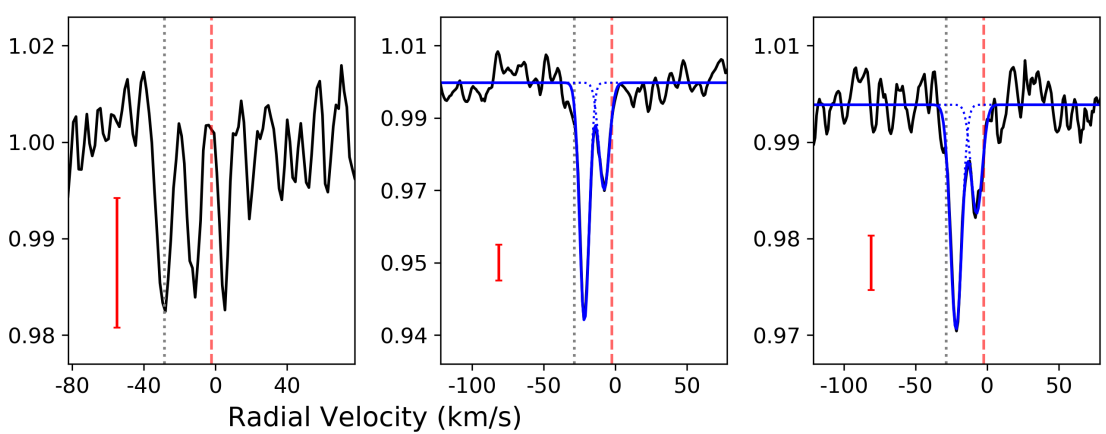

Fig. A.1. continued. 
I. Rebollido et al.: Exocomets: A spectroscopic survey

HD 146897
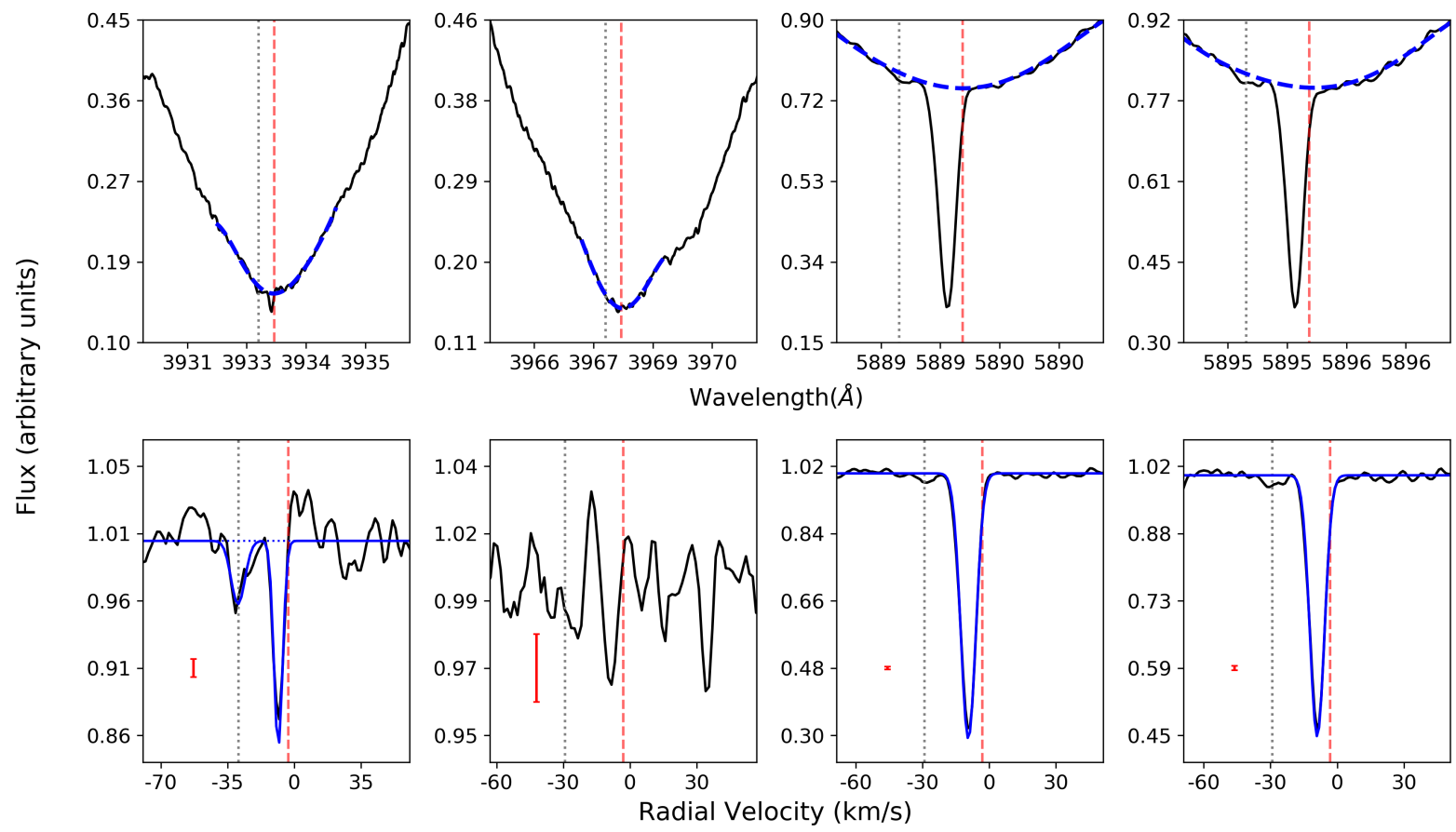

HD 147137
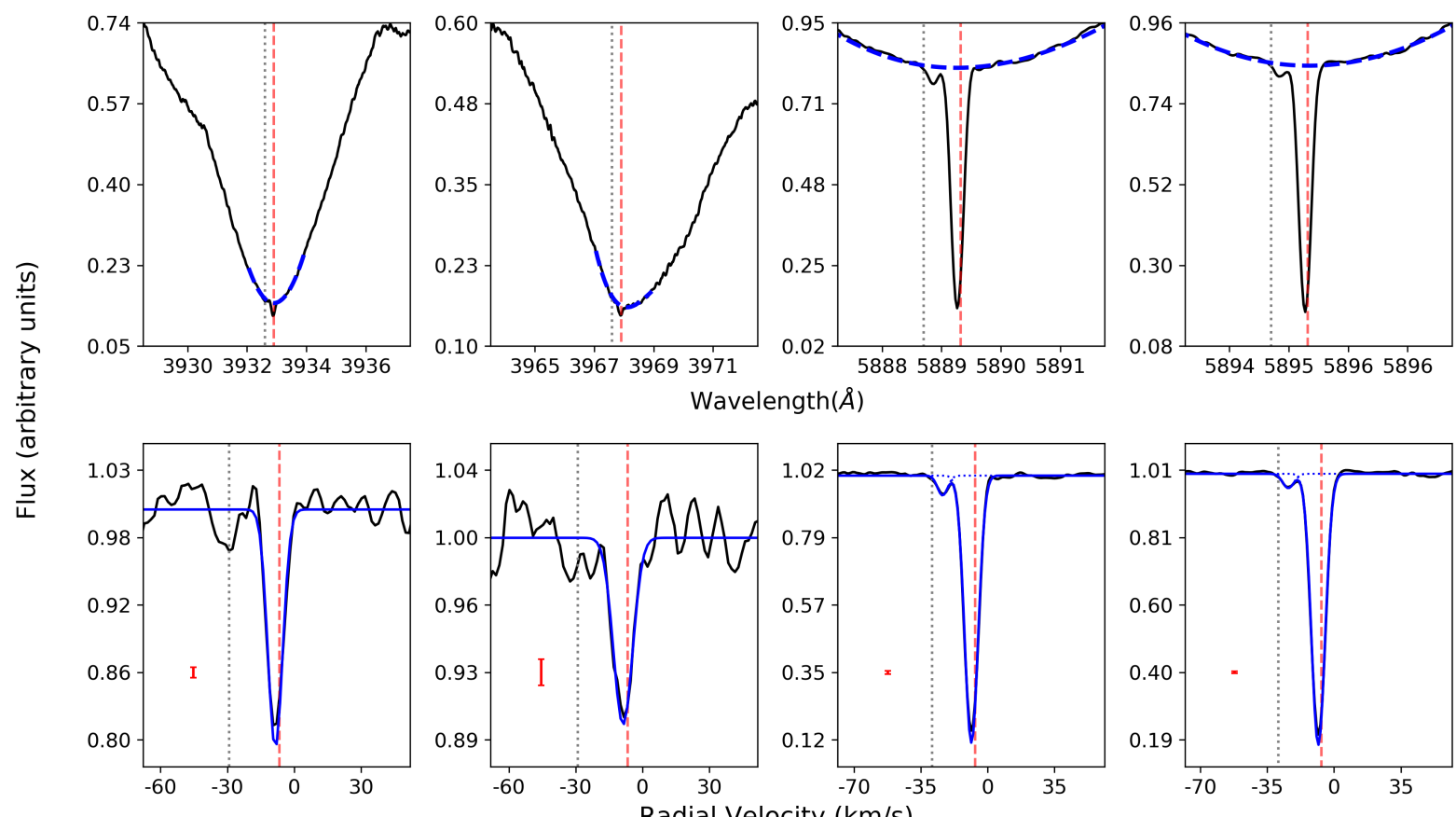

Wavelength $(\AA)$
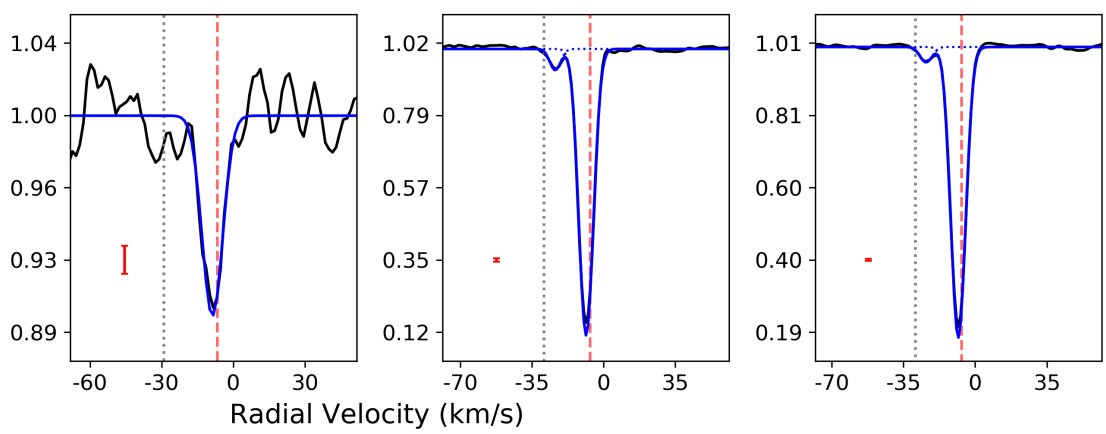

Fig. A.1. continued. 
HD 147220
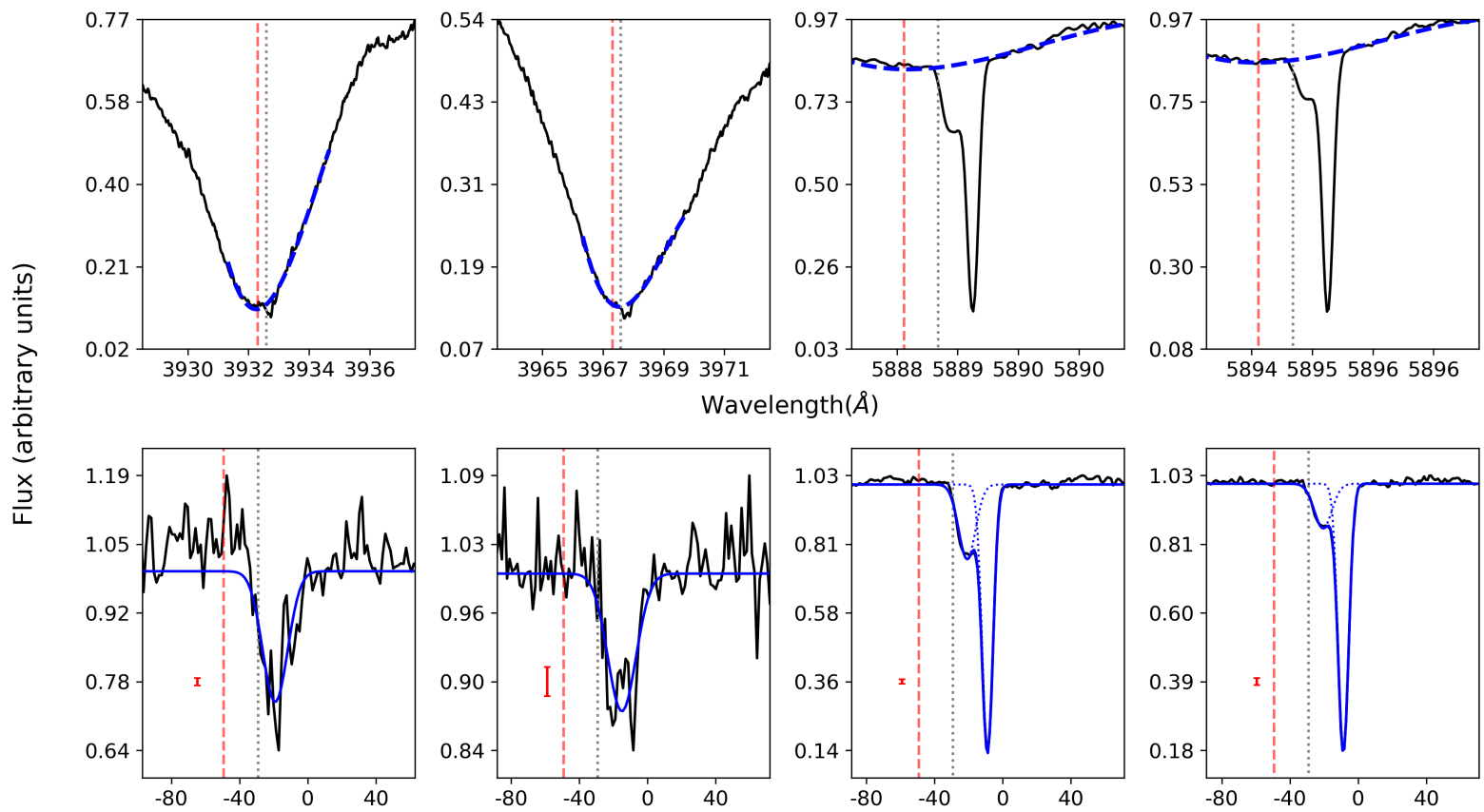

Wavelength $(\AA)$
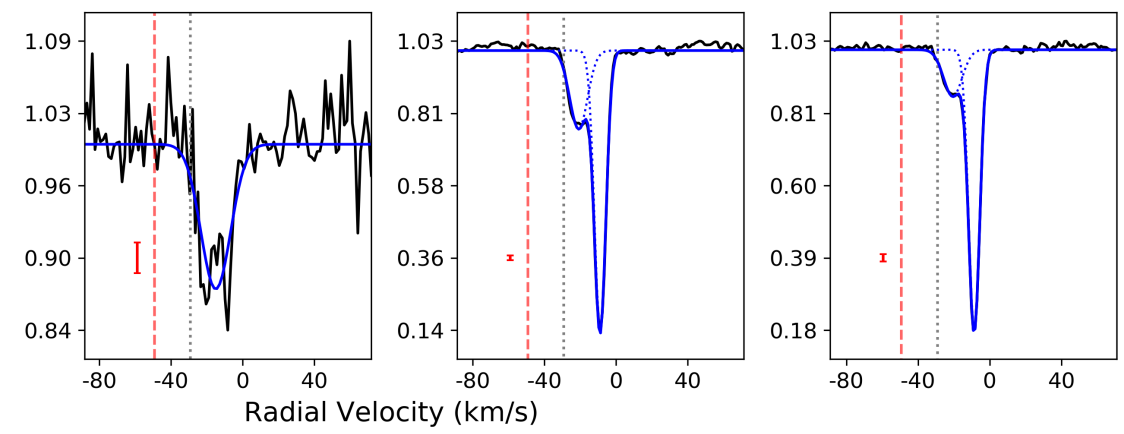

HD 148283
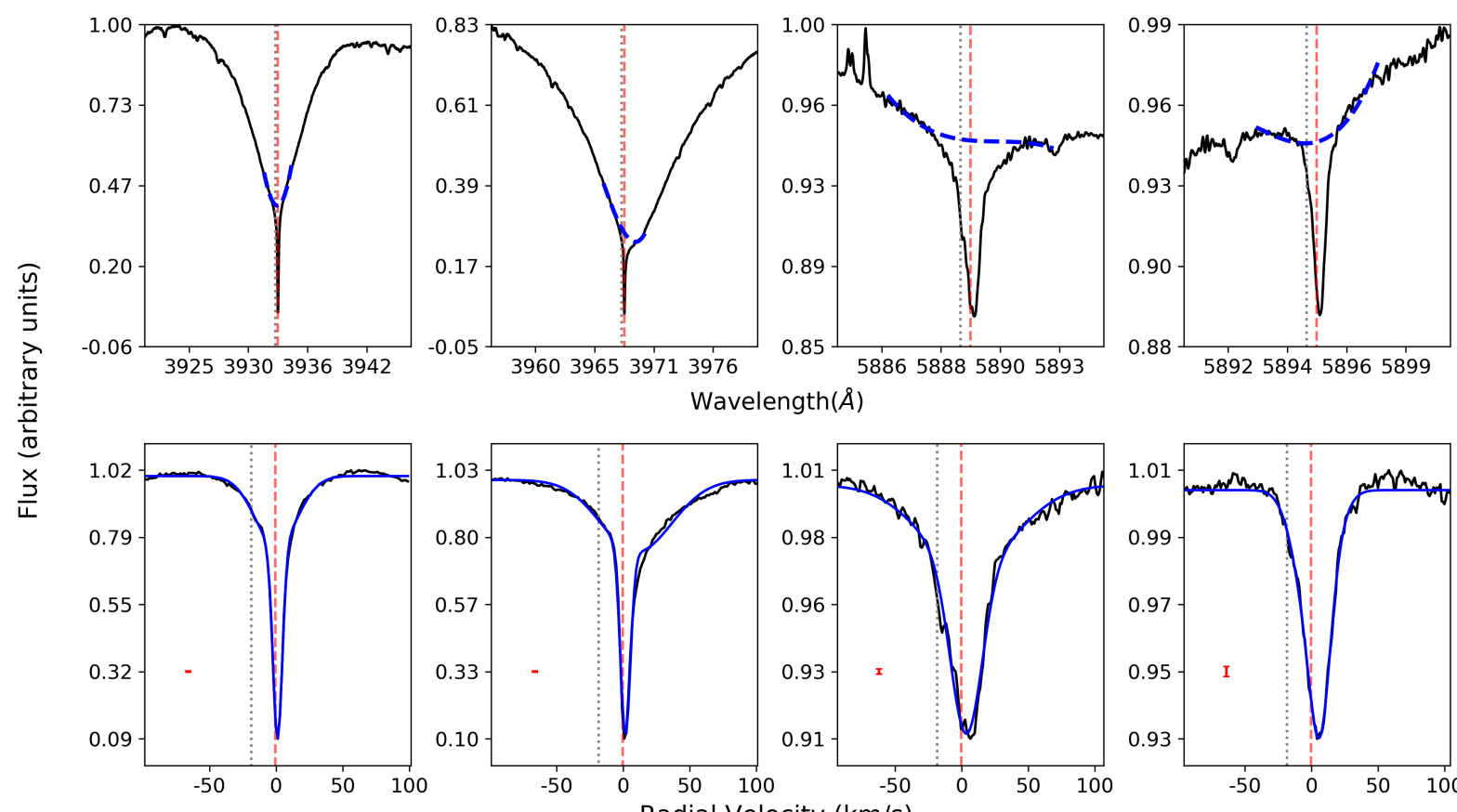

Wavelength $(\AA)$
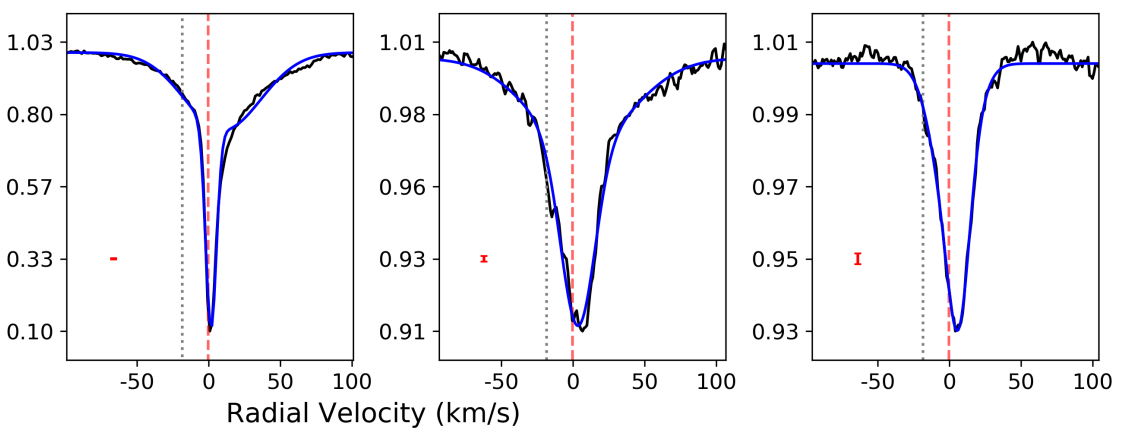

Fig. A.1. continued. 
HD 156623
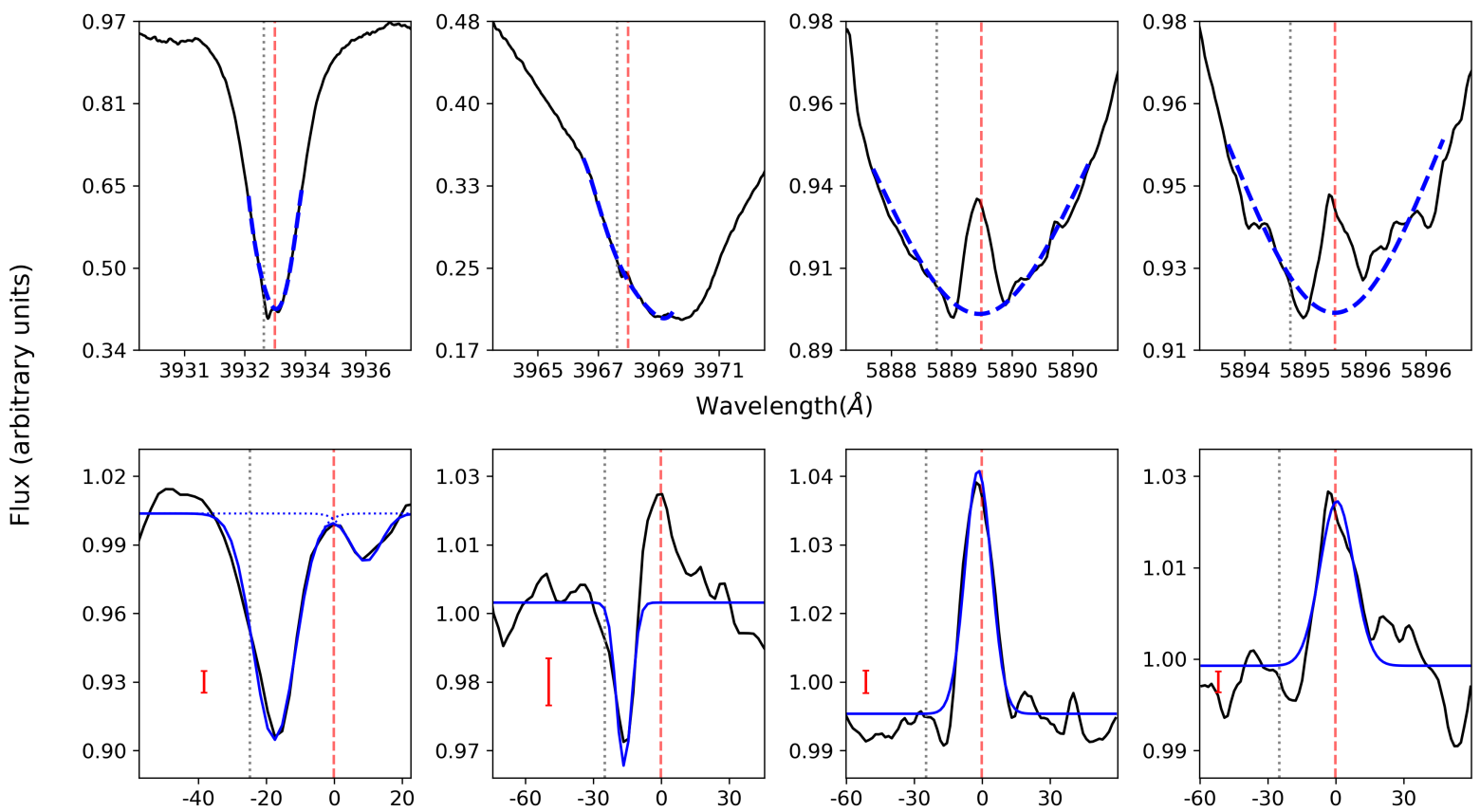
Wavelength $(\AA)$
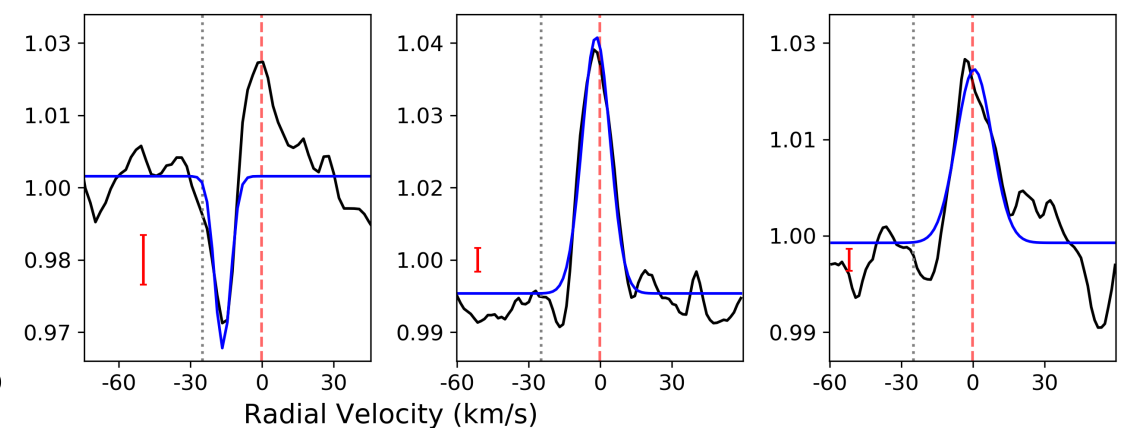

HD 158352
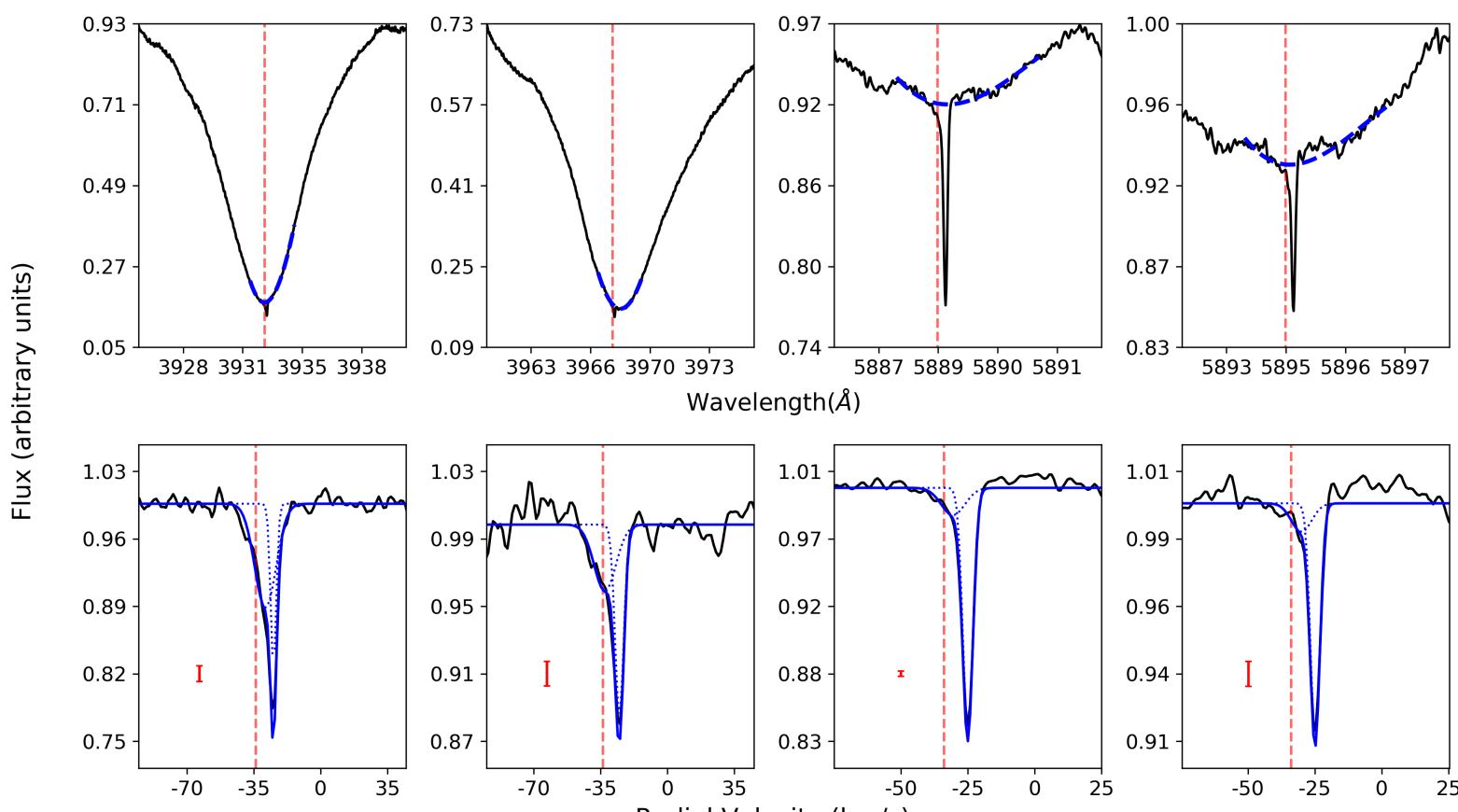

Wavelength $(\AA)$
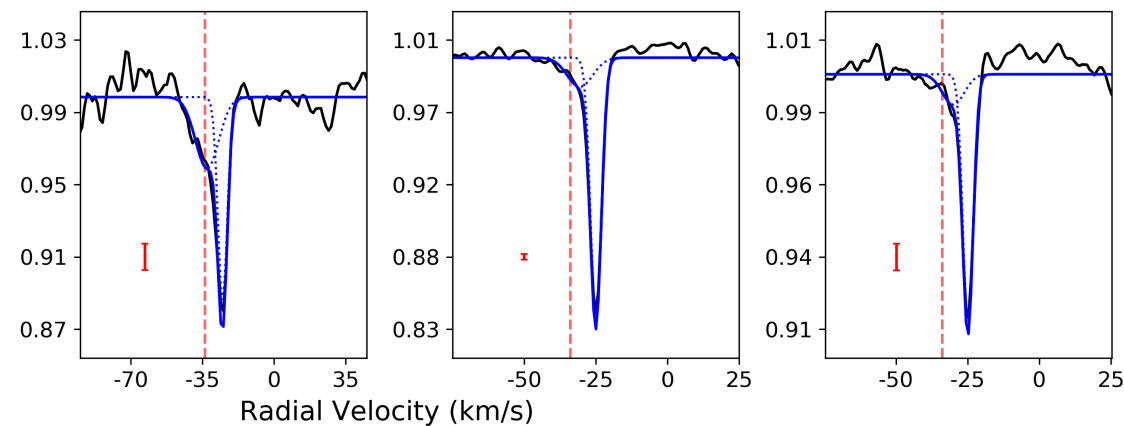

Fig. A.1. continued. 
HD 168646
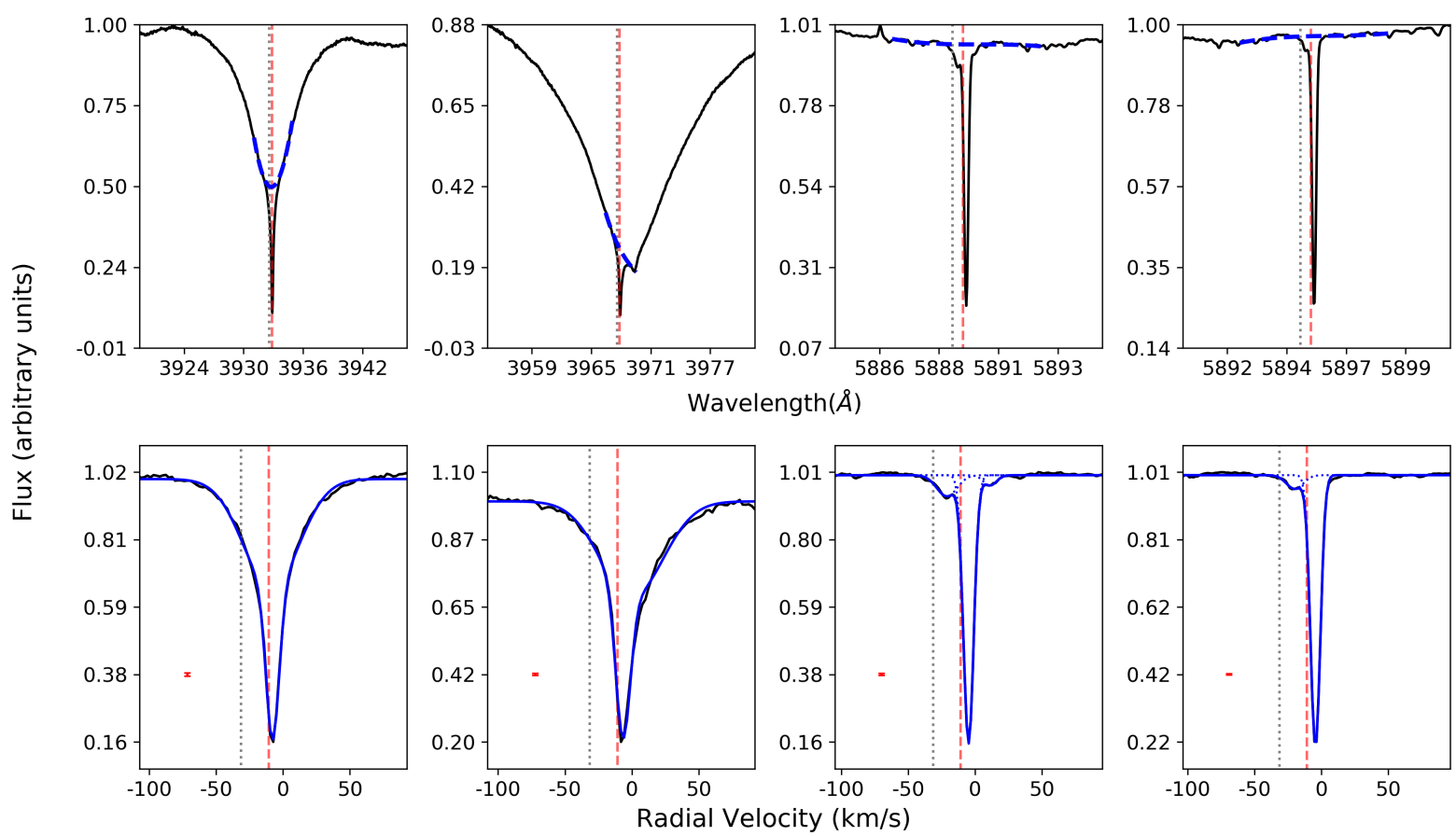

HD 172555
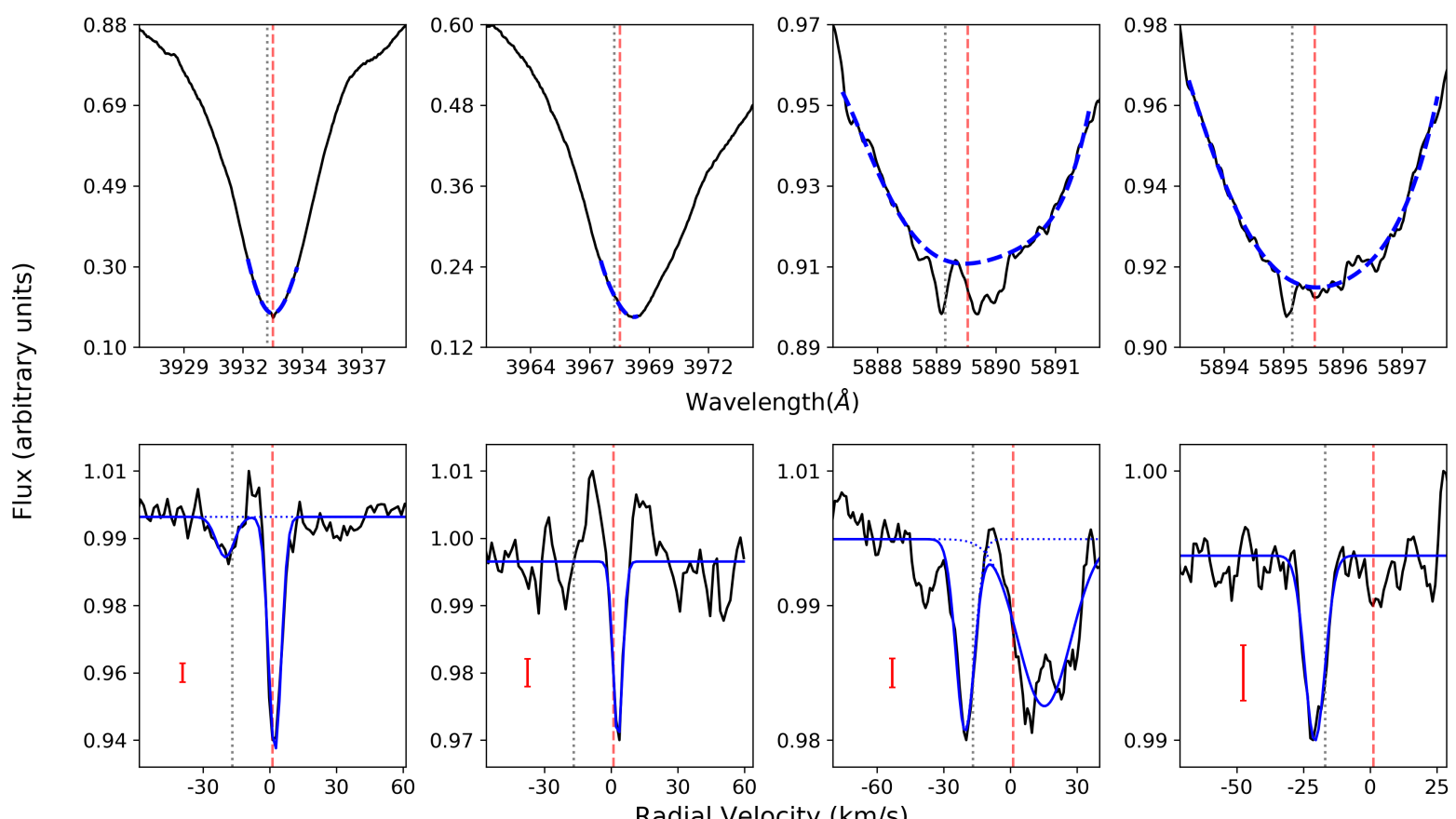

Wavelength $(\AA)$
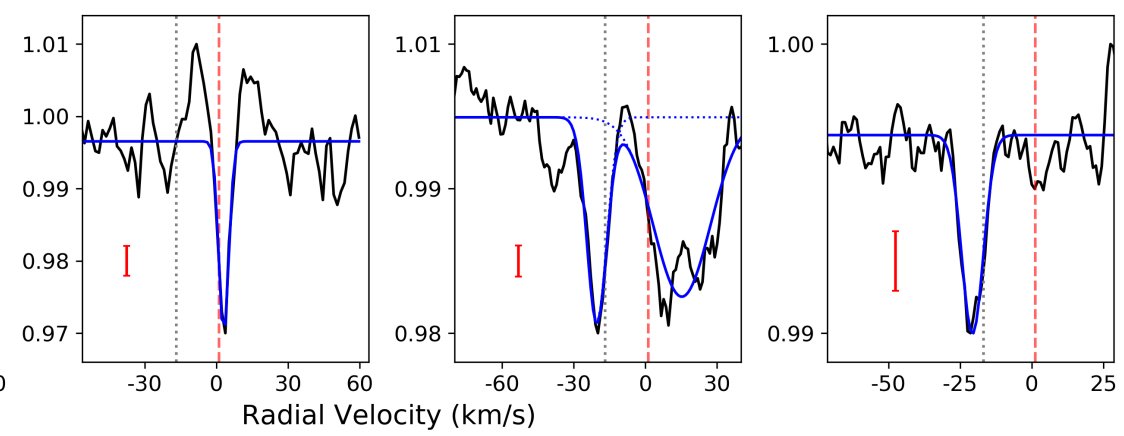

Fig. A.1. continued. 

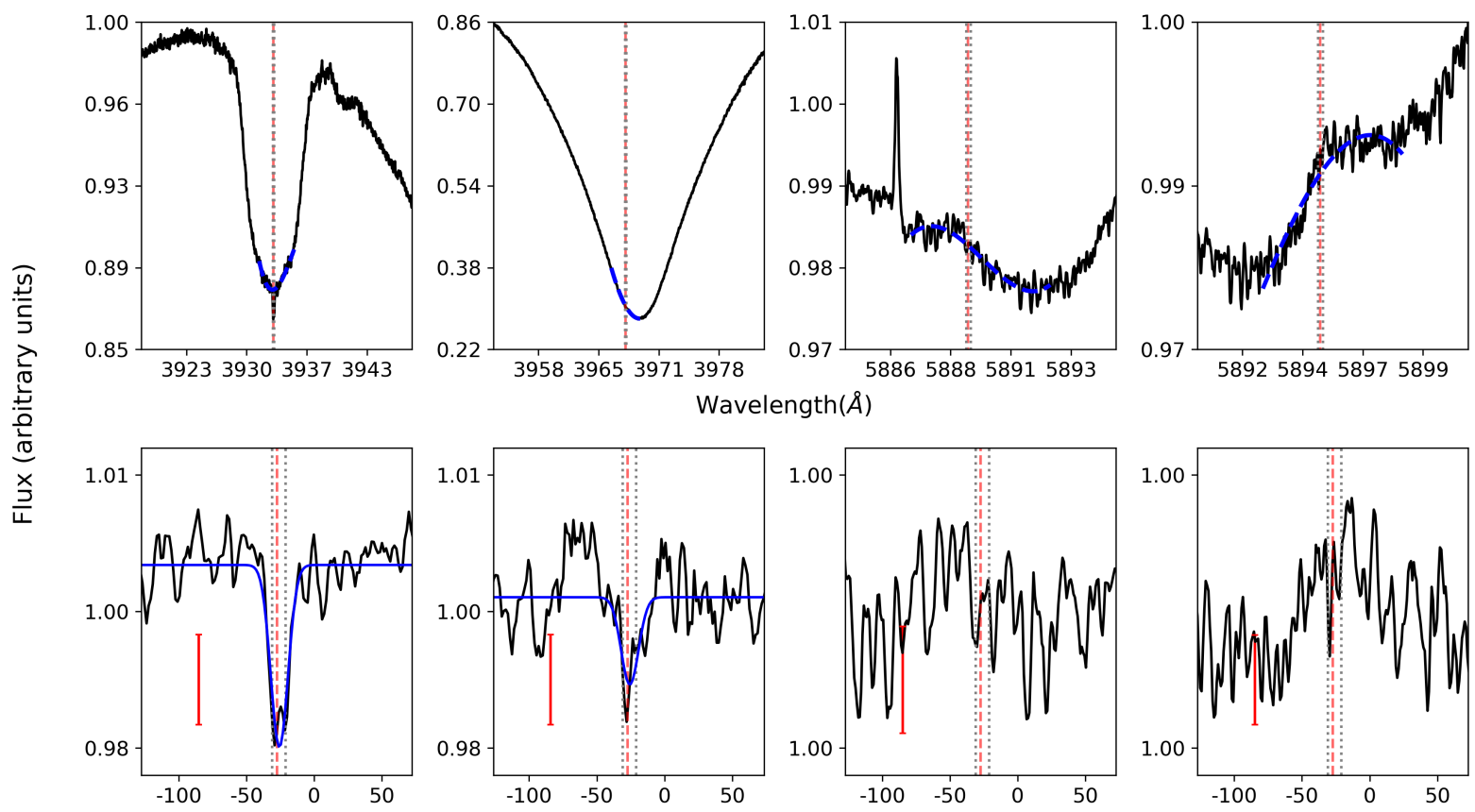
Wavelength $(\AA)$
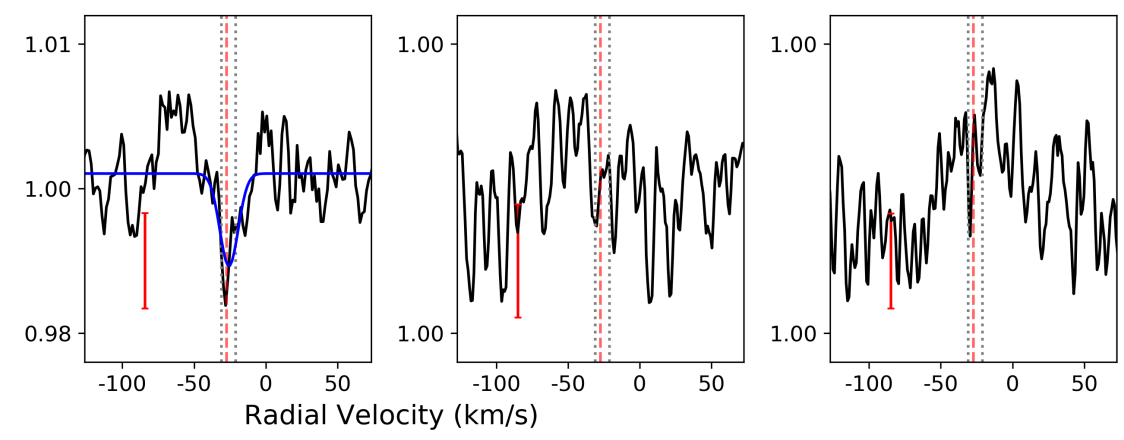

HD 181296
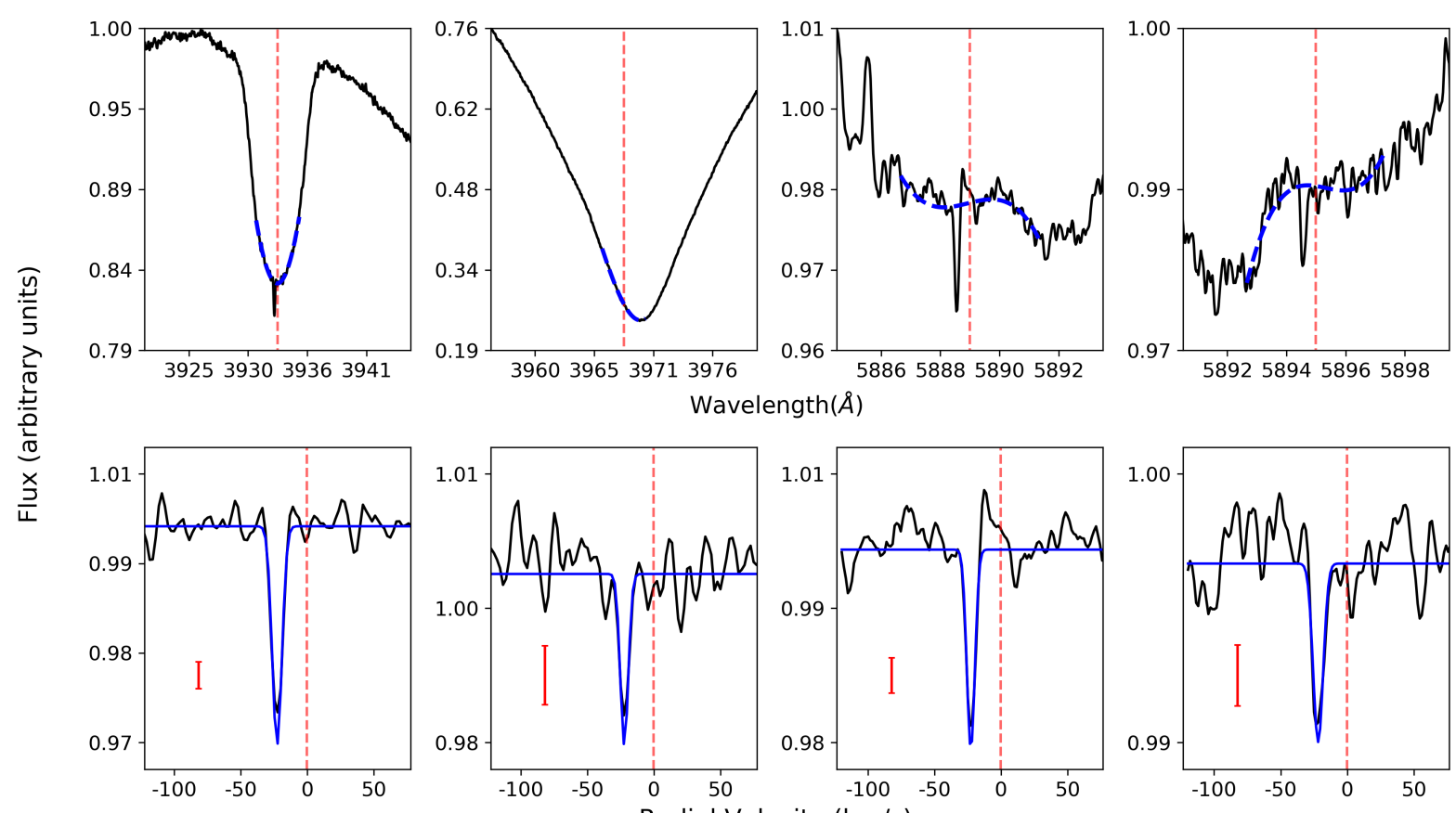
Wavelength $(\AA)$
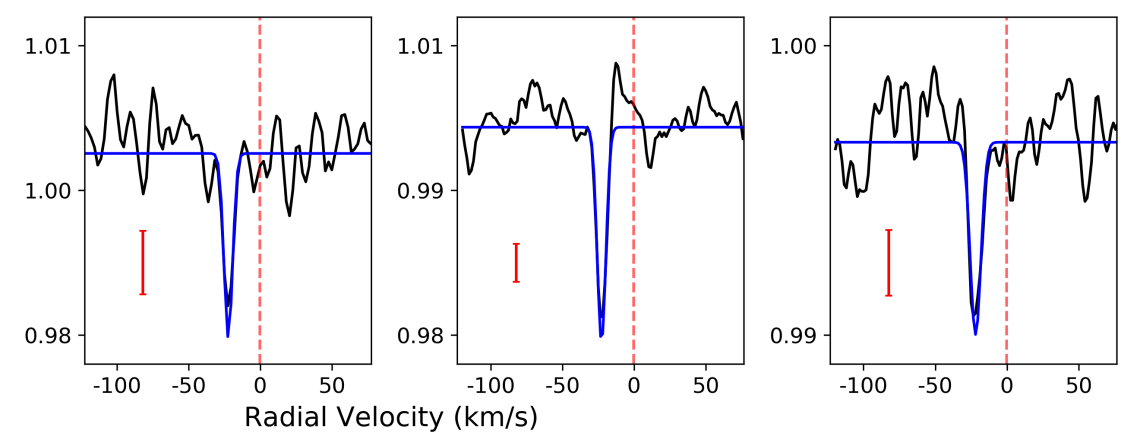

Fig. A.1. continued. 

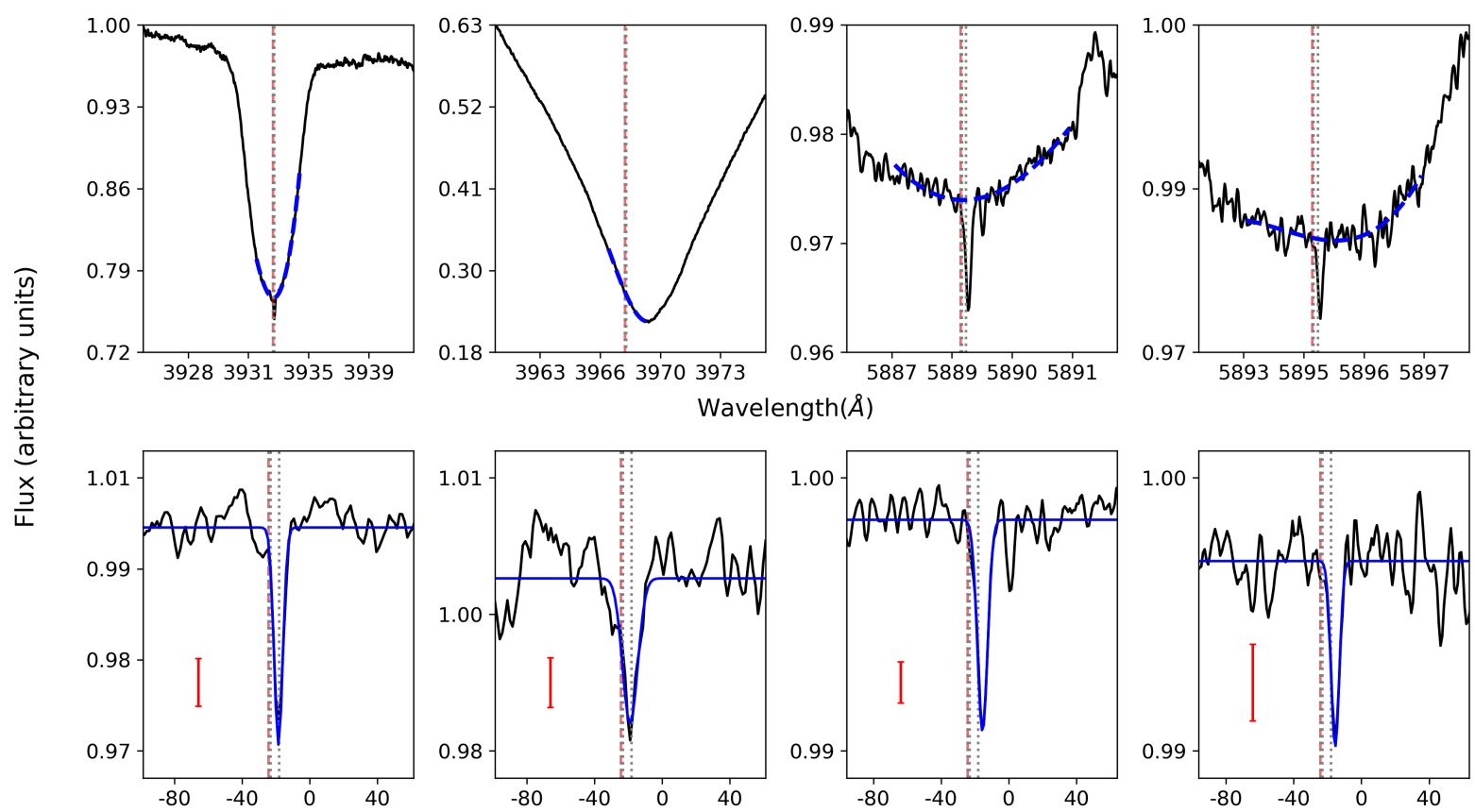
Wavelength $(\AA)$
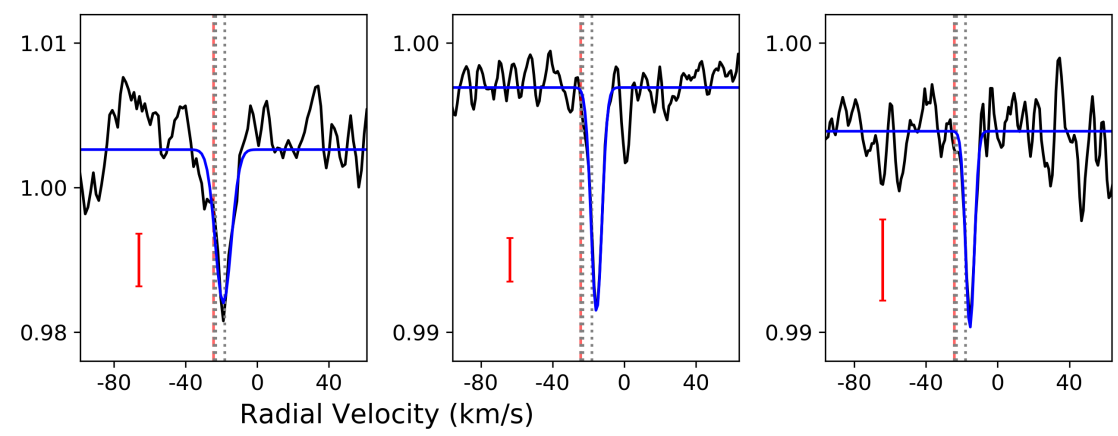

HD 188228
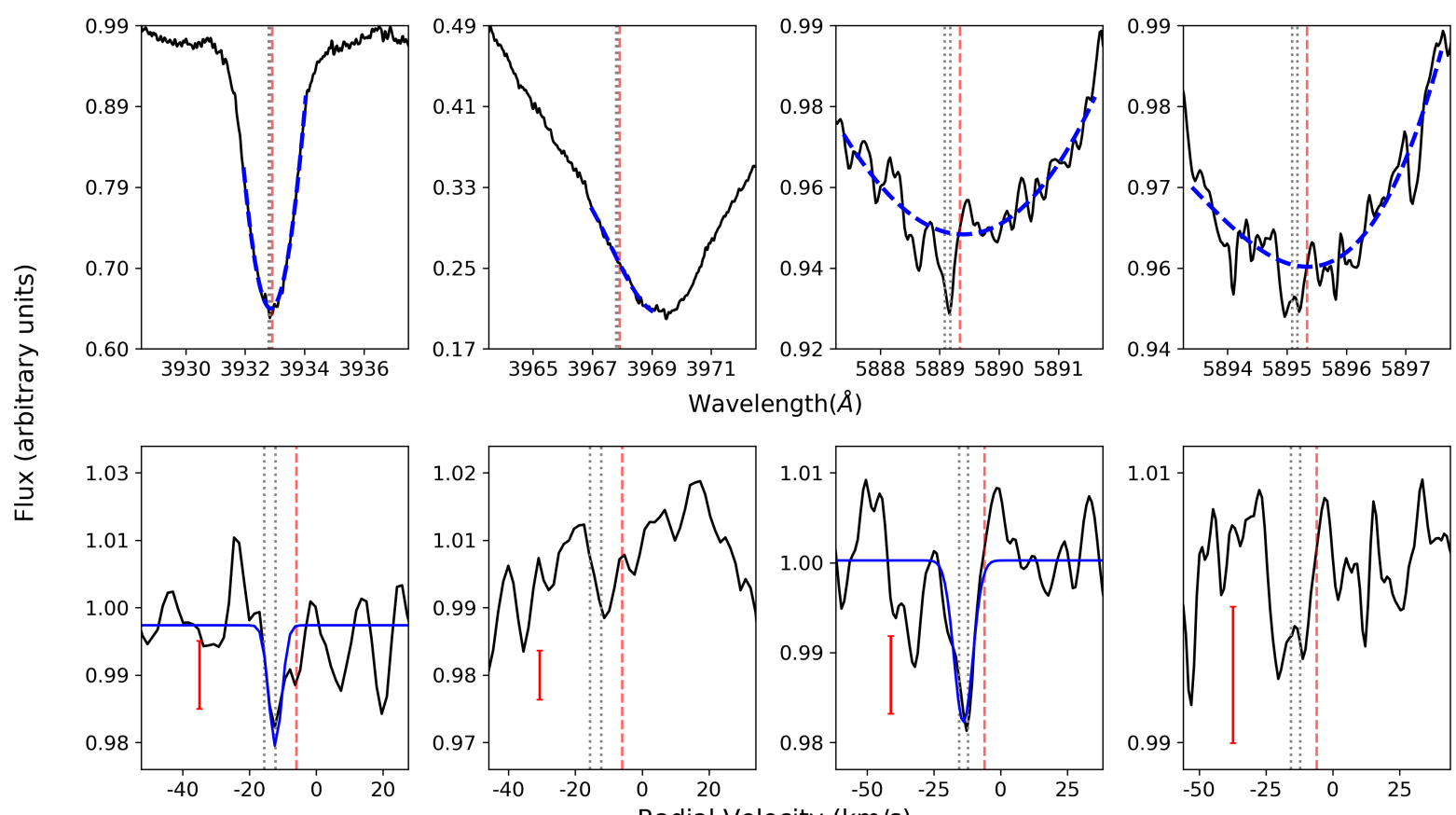
Wavelength $(\AA)$
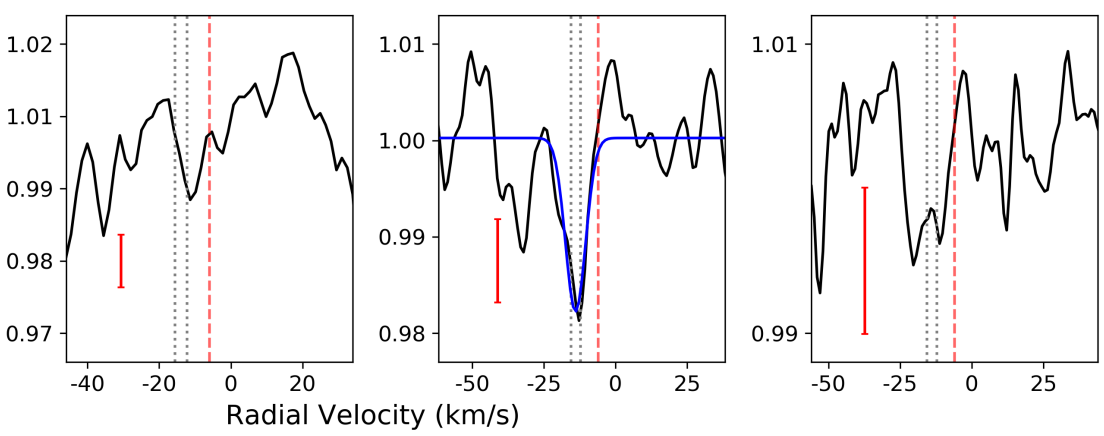

Fig. A.1. continued. 
I. Rebollido et al.: Exocomets: A spectroscopic survey

HD 192518
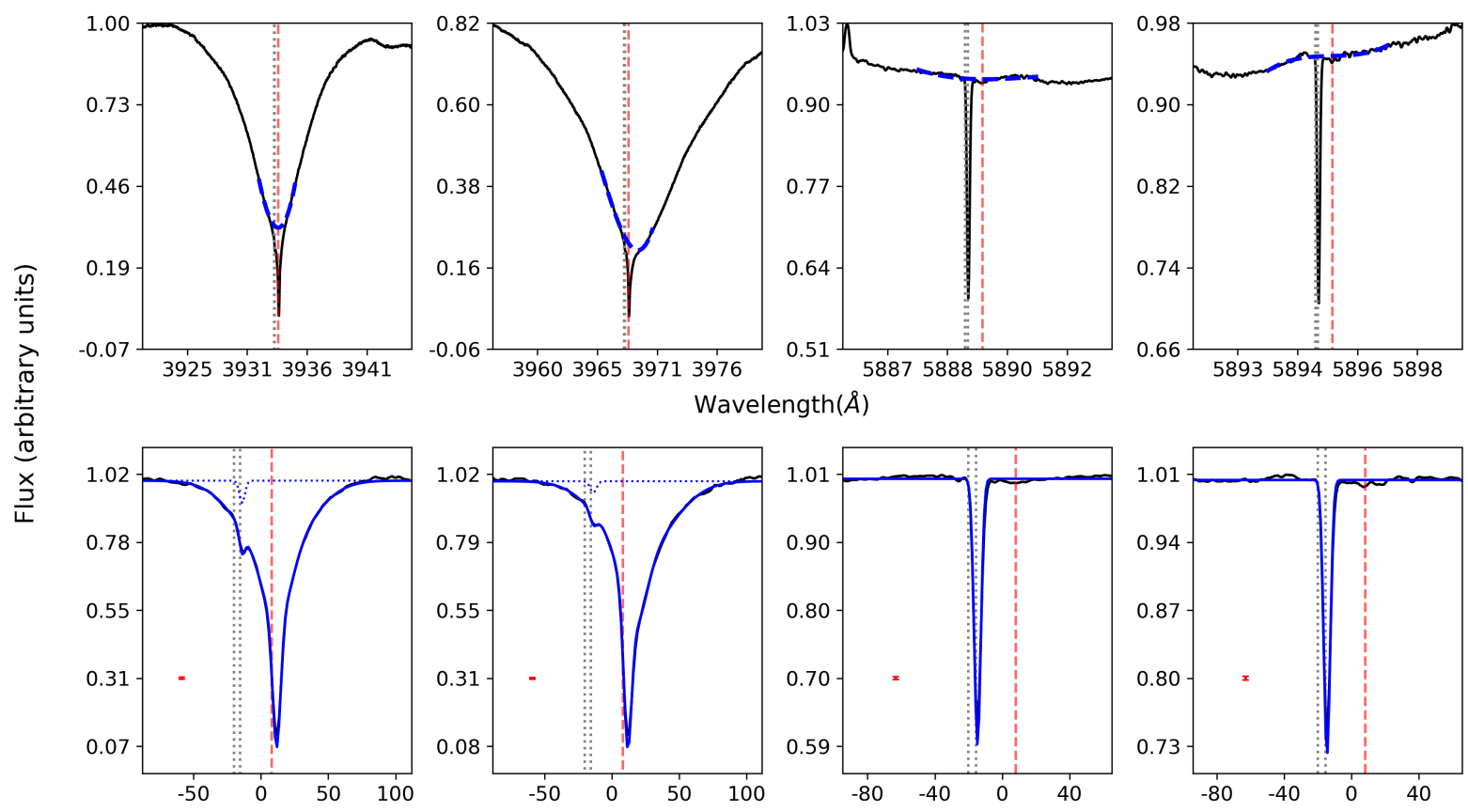

Wavelength $(\AA)$
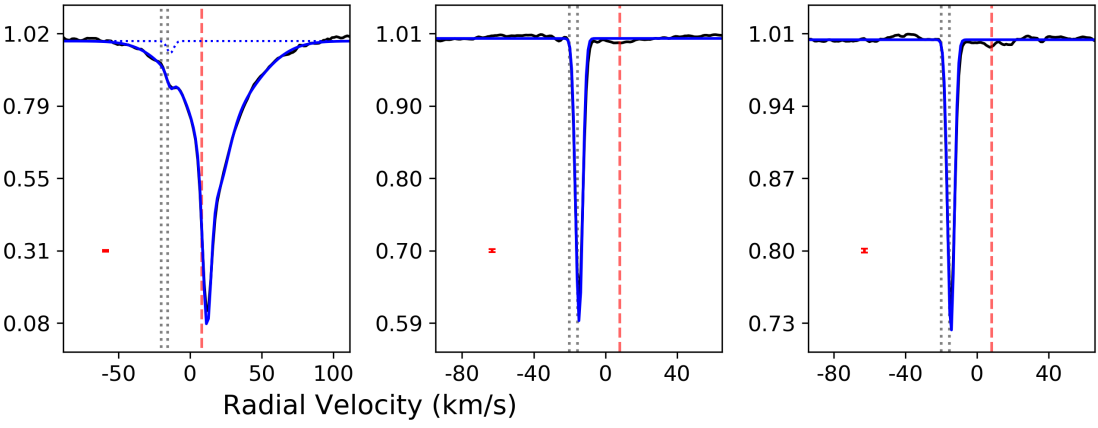

HD 196724
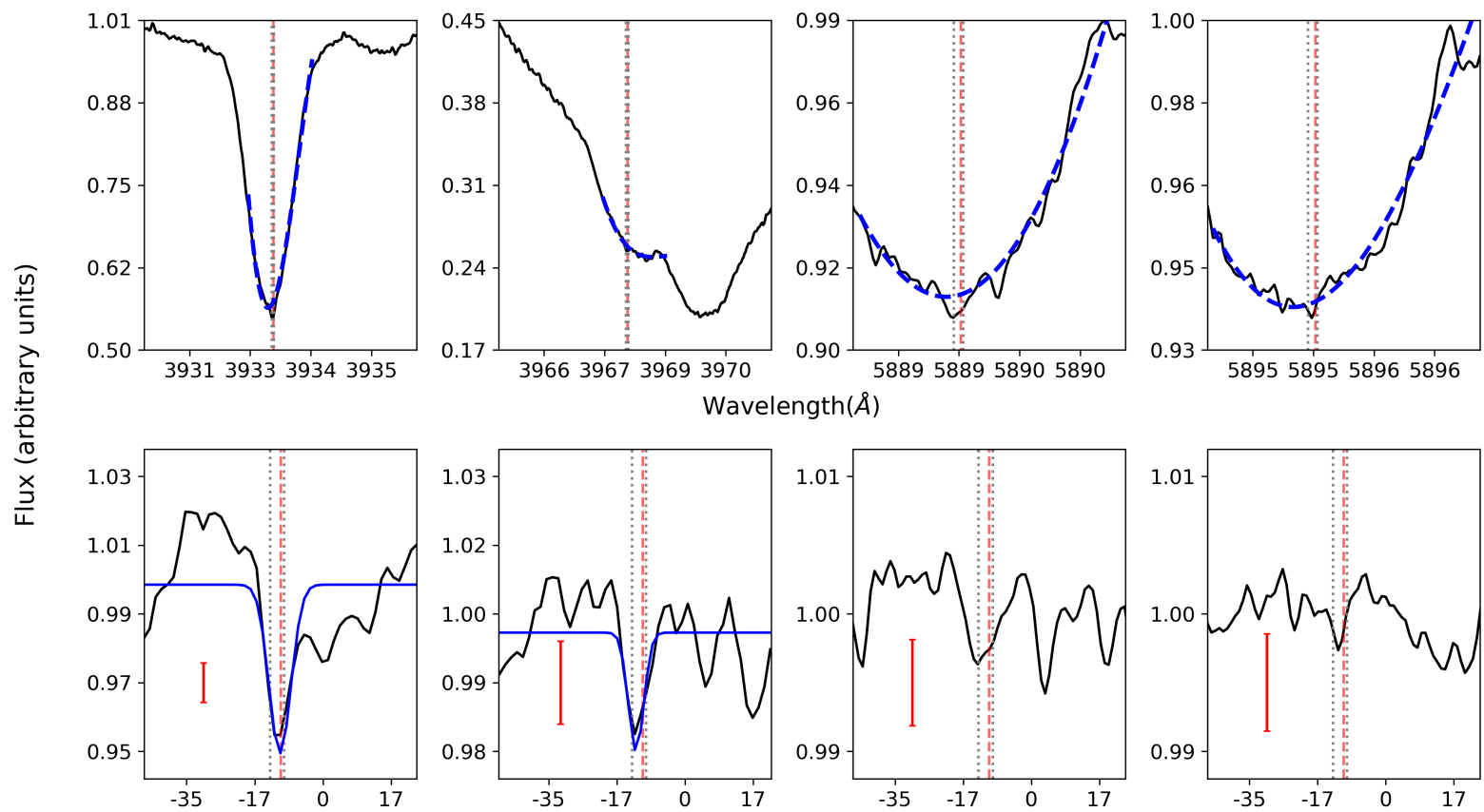
Wavelength $(\AA)$
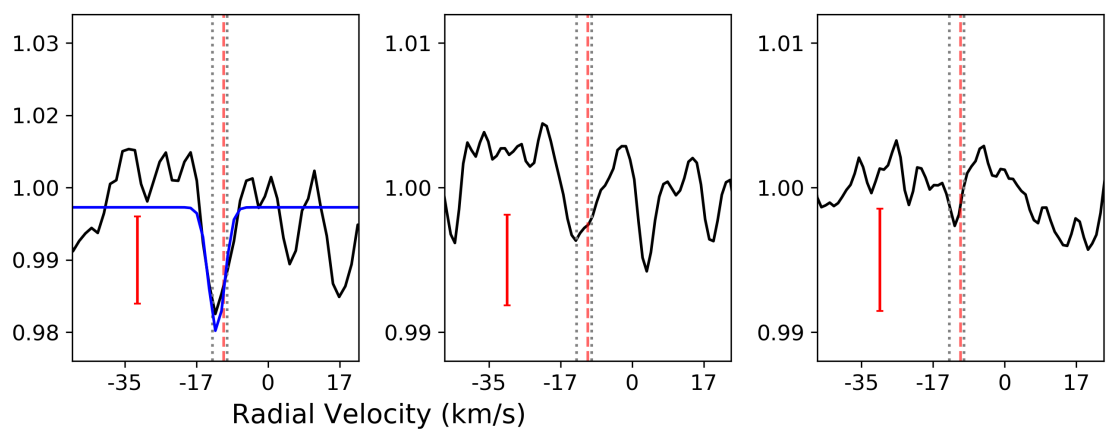

Fig. A.1. continued. 
HD 198160
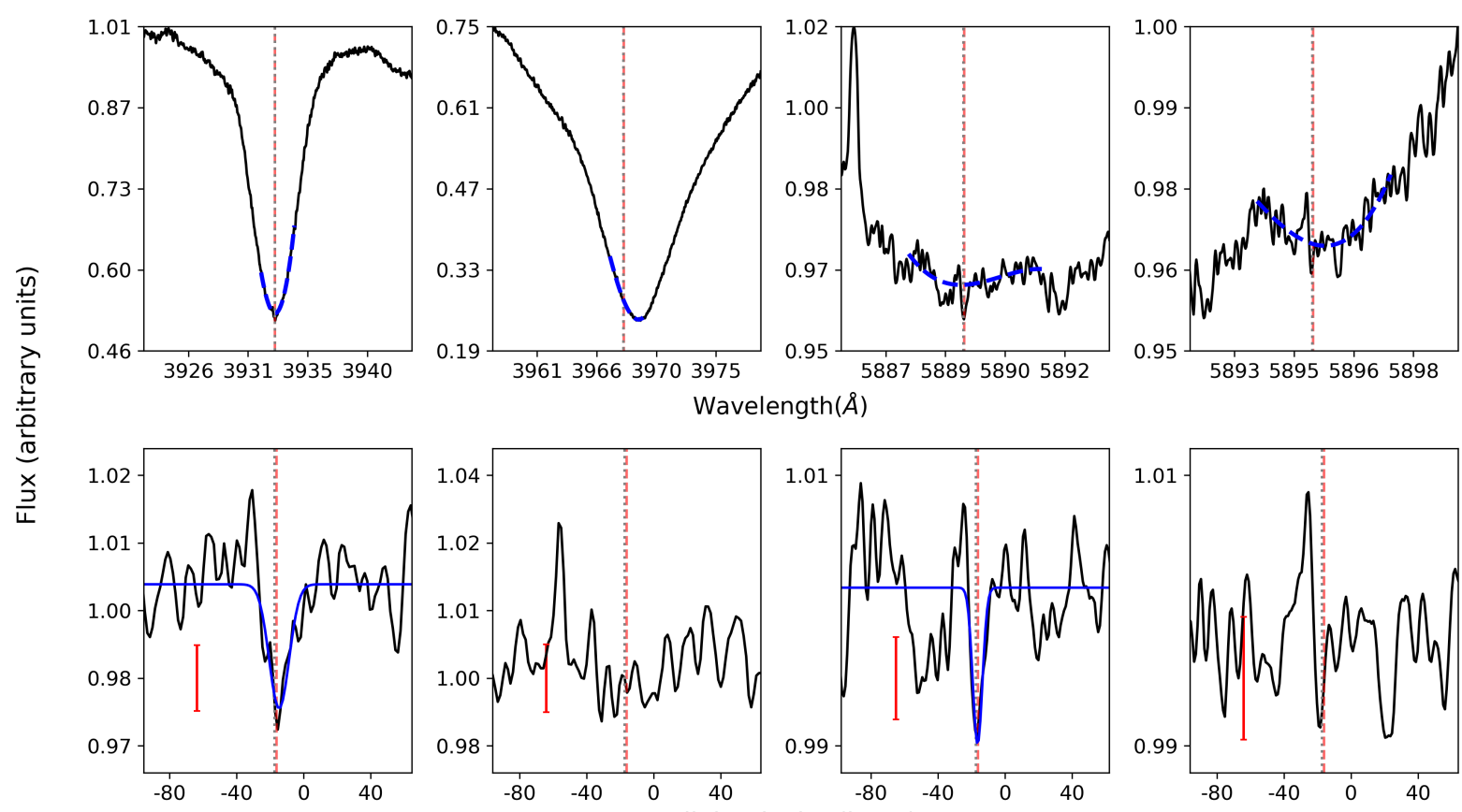

Wavelength $(\AA)$
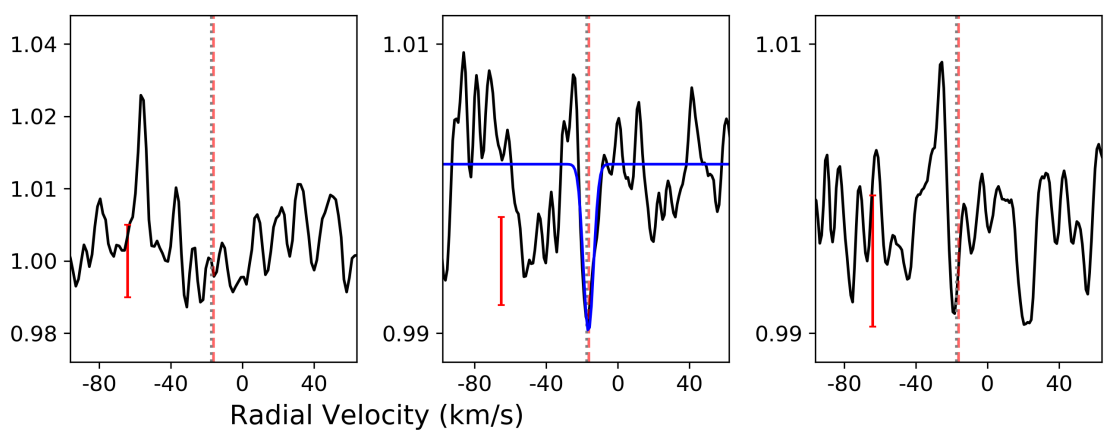

HD 198161
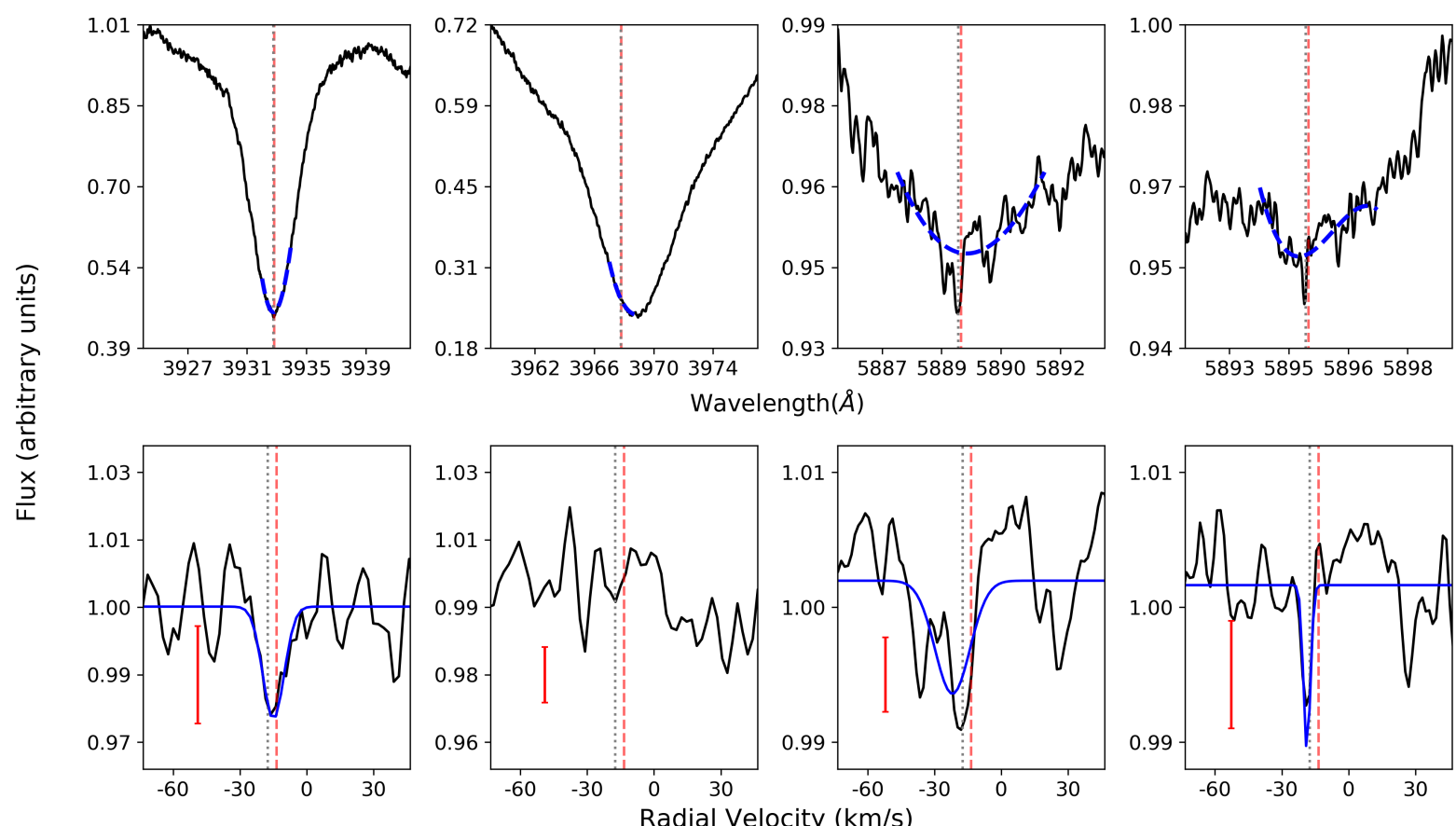

Wavelength $(\AA)$
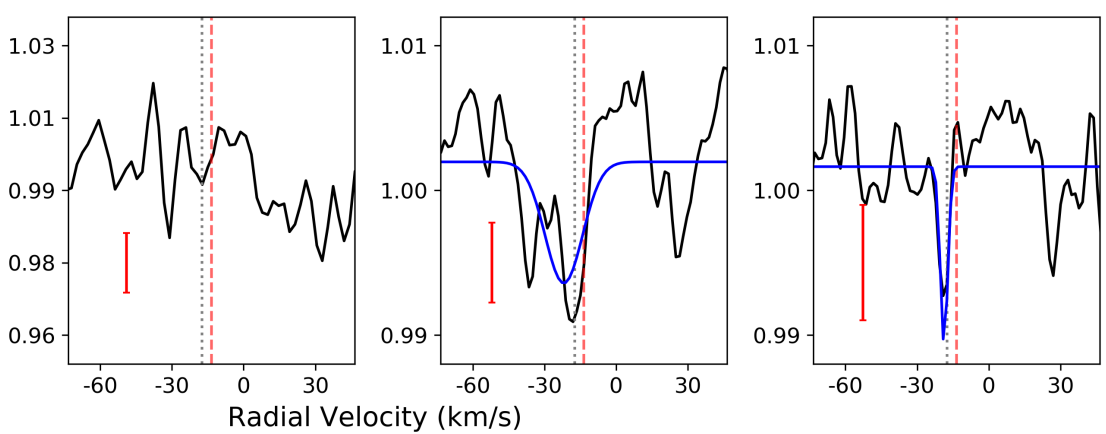

Fig. A.1. continued. 
I. Rebollido et al.: Exocomets: A spectroscopic survey

HD 210418
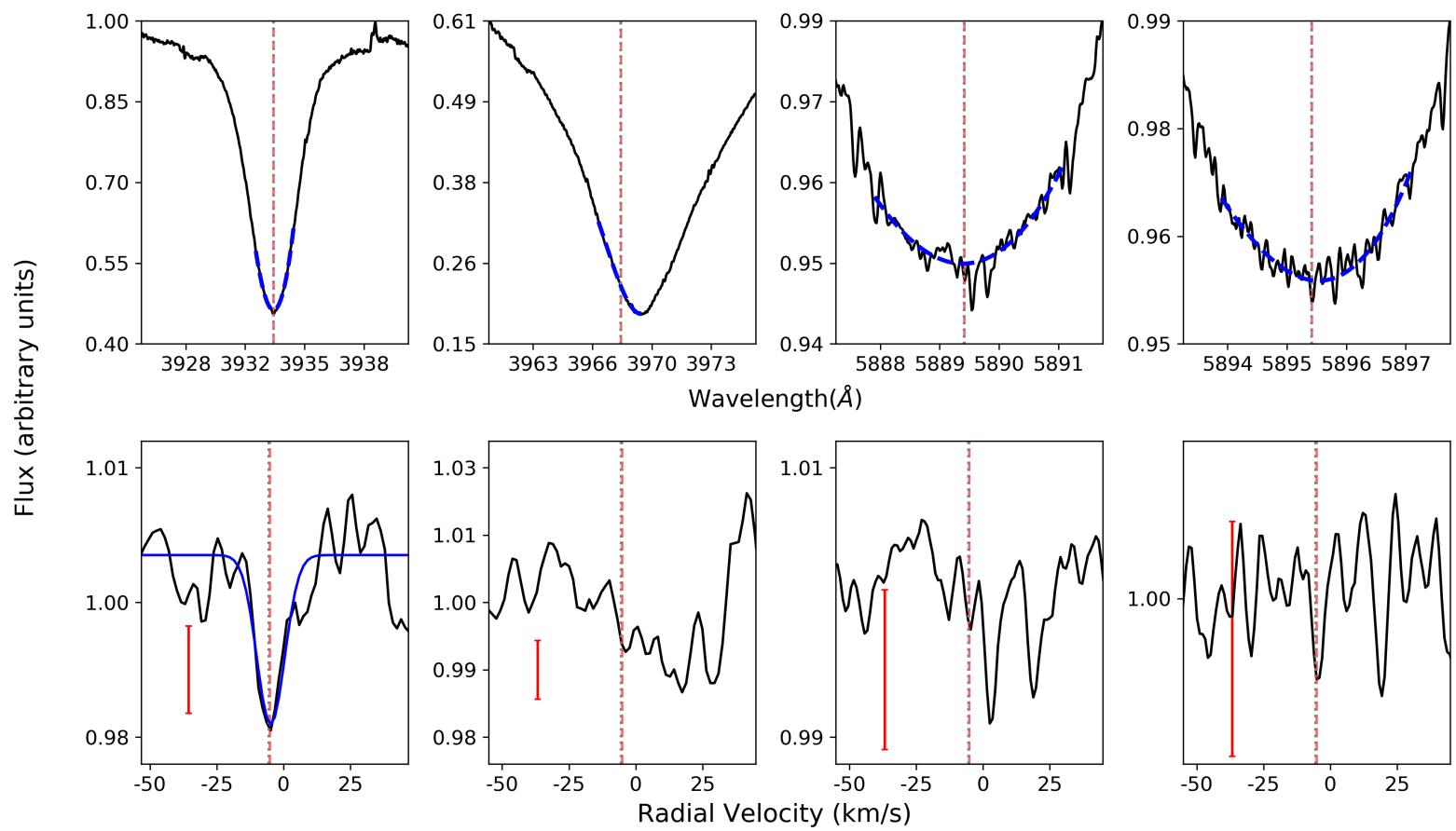

HD 217782
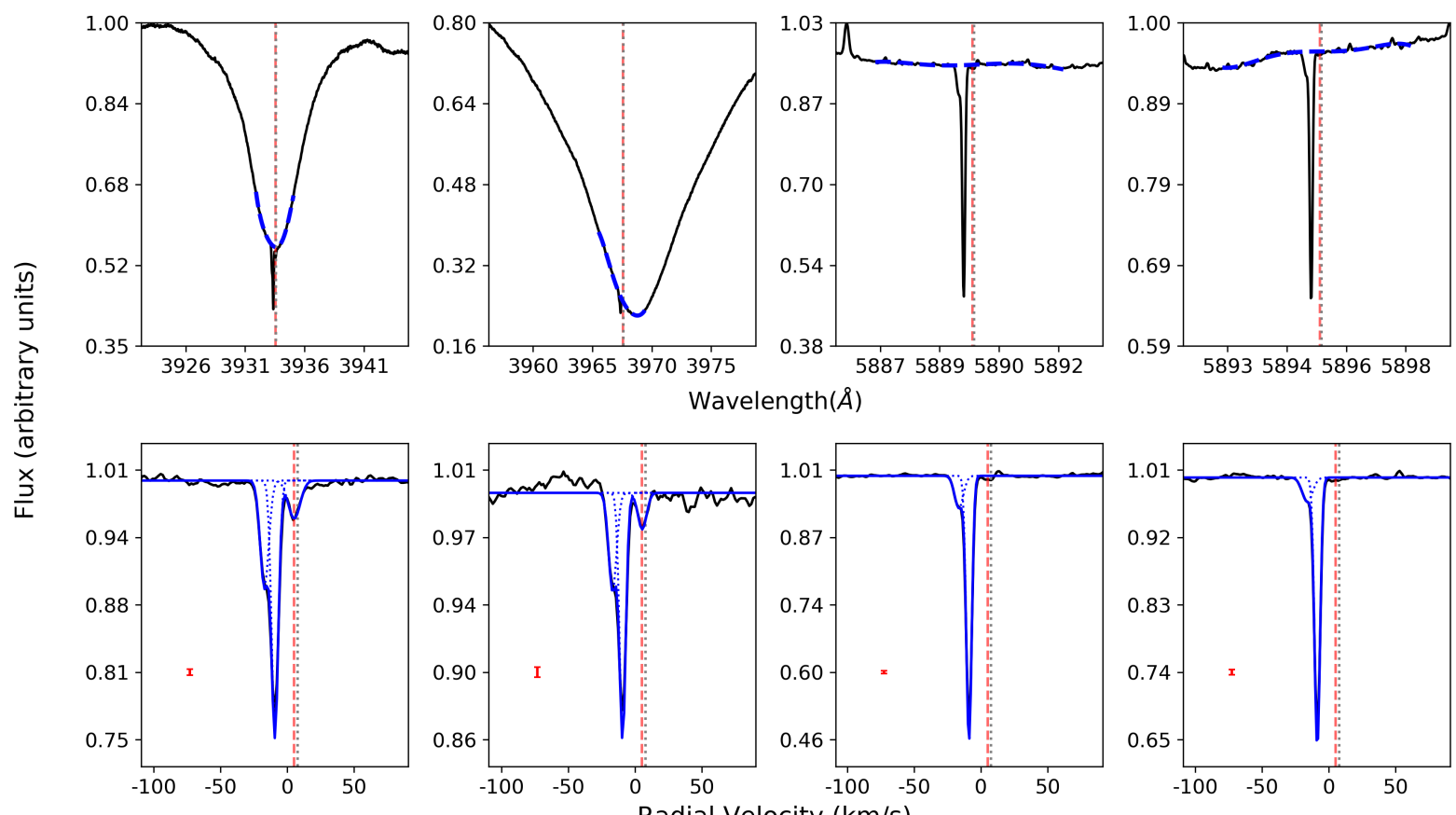

Wavelength $(\AA)$
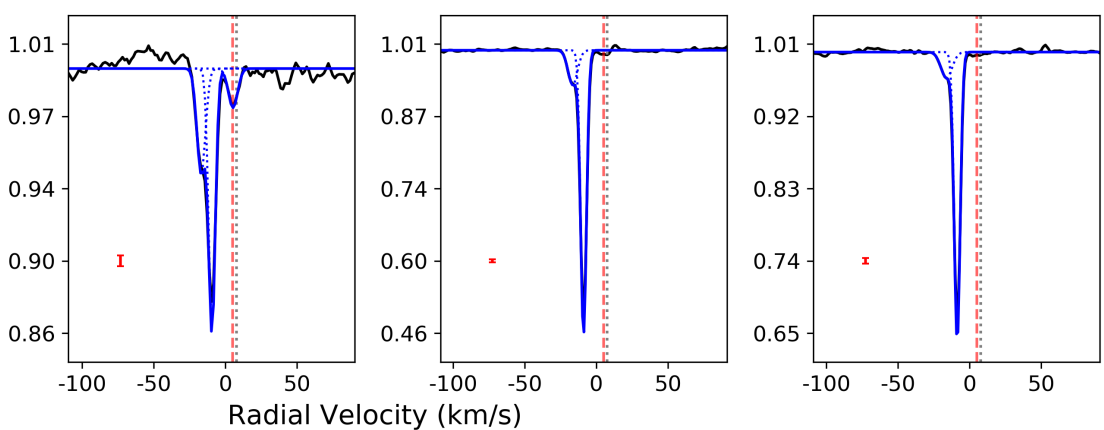

Fig. A.1. continued. 
HD 221756
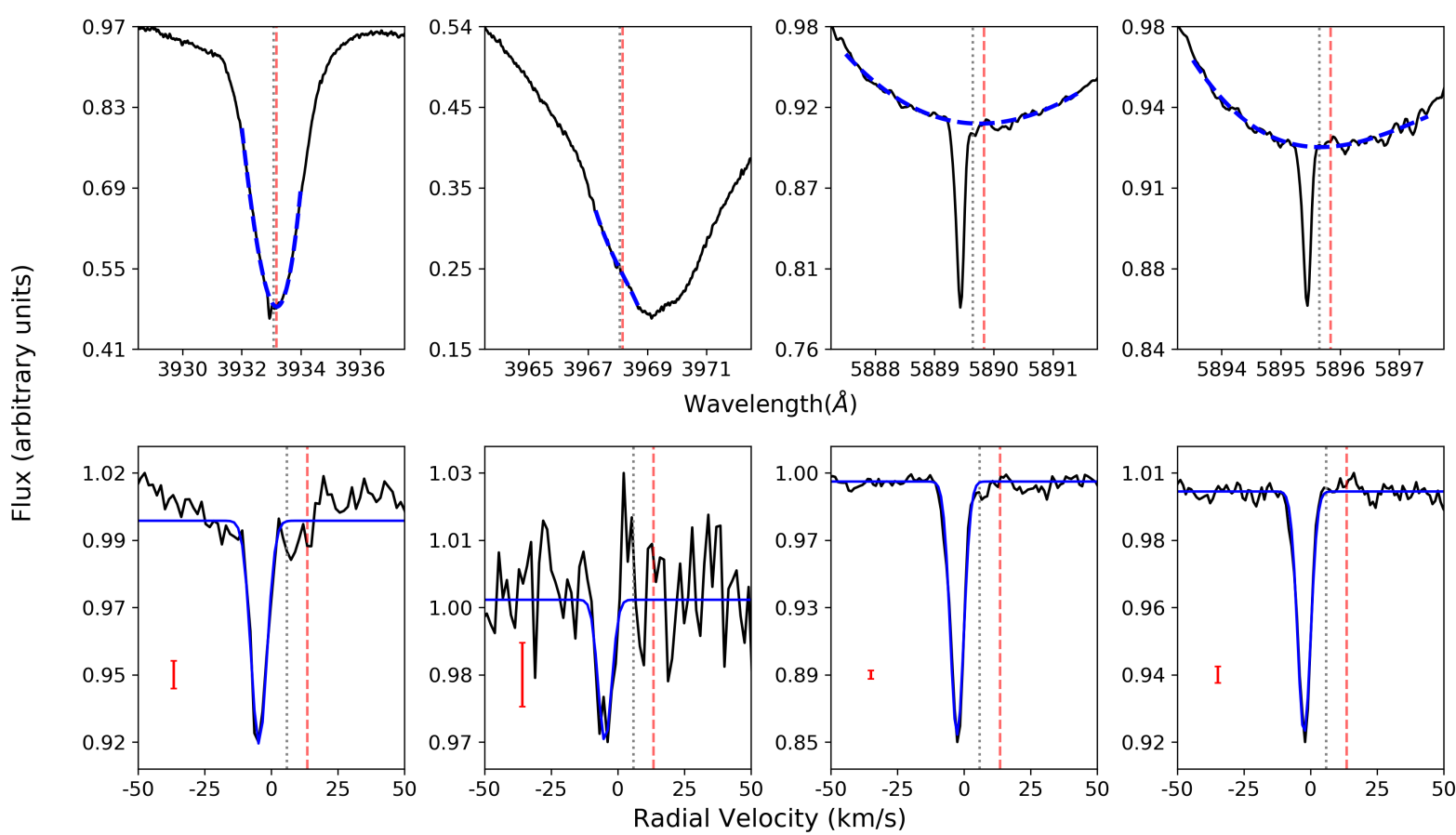

HD 224392
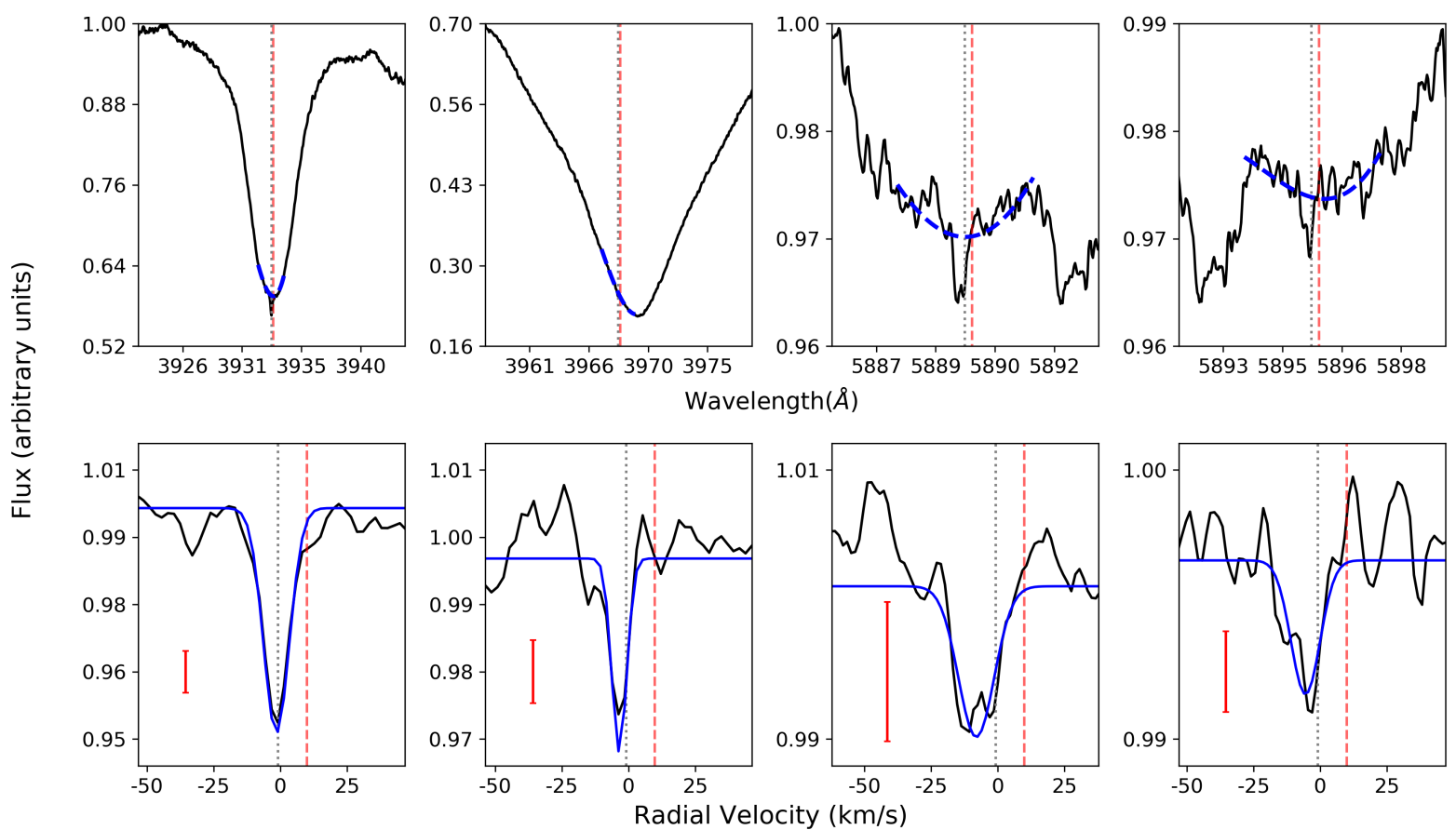

Fig. A.1. continued. 


\section{Appendix B: Additional tables}

Table B.1. Sample of observed stars.

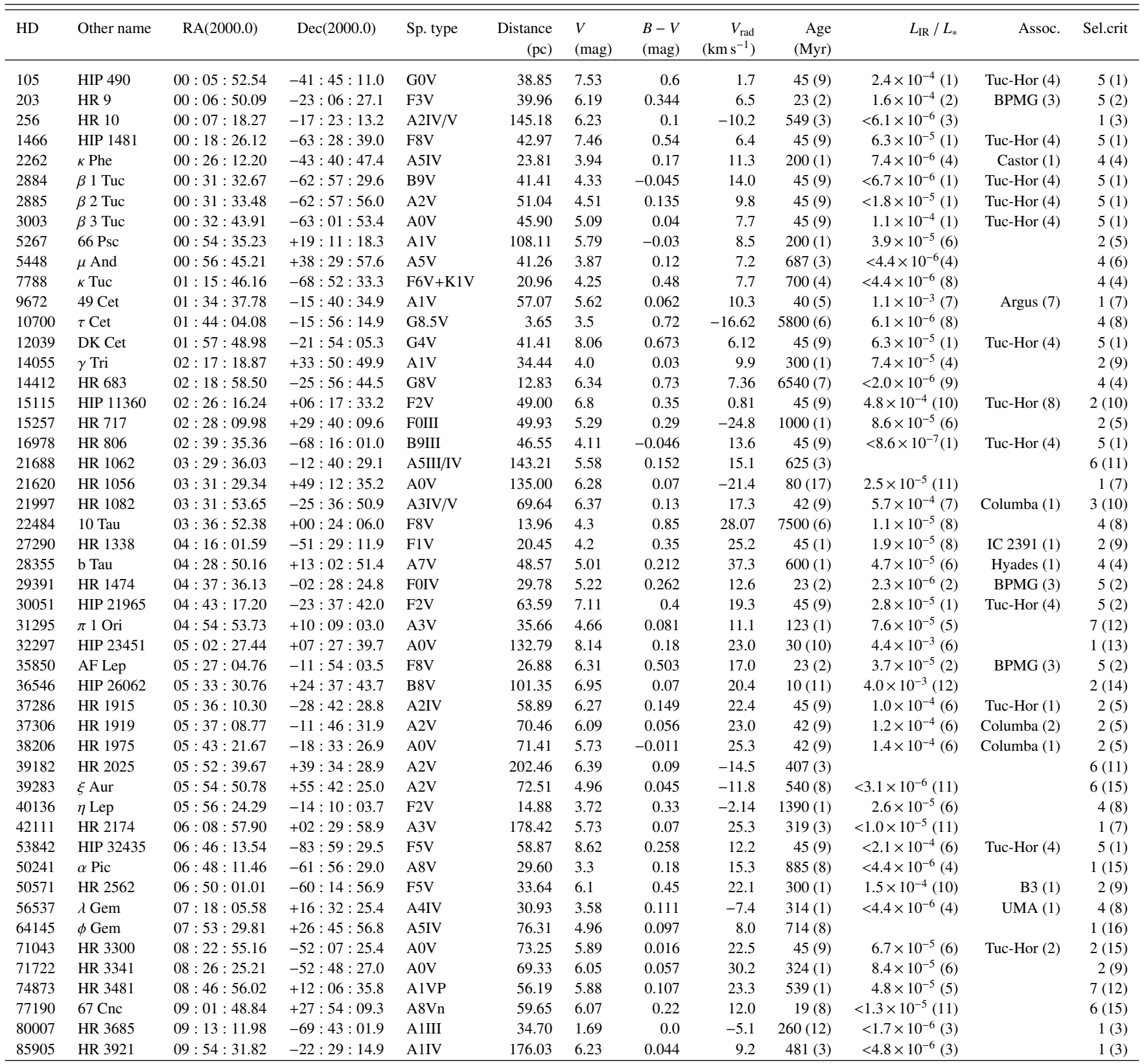

Notes. Columns are self-explanatory. Numbers within parenthesis in Cols. 10 to 13 denote the corresponding references. Column 13 specifies the primary selection criteria as described in the text: 1 . Previously detected exocomets; 2 . Debris discs; 3 . Debris discs with cold gas; 4 . Near-infrared excesses; 5. Young discs; 6. Shell stars; 7. $\lambda$ Boo stars.

References. For age: (1) Chen et al. (2014); (2) Mamajek \& Bell (2014); (3) Gontcharov (2012); (4) Gáspár et al. (2013); (5) Torres et al. (2008); (6) Maldonado et al. (2012); (7) Eiroa et al. (2013); (8) David \& Hillenbrand (2015); (9) Bell et al. (2015); (10) Kalas (2005); (11) Lisse et al. (2017); (12) Su et al. (2006); (13) Marino et al. (2017); (14) Pecaut \& Mamajek (2016); (15) Bochanski et al. (2018); (16) Pecaut et al. (2012); (17) Roberge \& Weinberger (2008); References for $L_{\mathrm{IR}} / L_{\star}$ (1) Donaldson et al. (2012); (2) Riviere-Marichalar et al. (2014); (3) Redfield et al. (2007) ; (4) Thureau et al. (2014); (5) Draper et al. (2016); (6) Chen et al. (2014); (7) Moór et al. (2015b); (8) Sibthorpe et al. (2018); (9) Eiroa et al. (2013); (10) Moór et al. (2011a); (11) Roberge \& Weinberger (2008); (12) Lisse et al. (2017); (13) Currie et al. (2011)5; (14) Duchêne et al. (2014); (15) Riviere-Marichalar et al. (2013); (16) Vican et al. (2016); (17) Moór et al. (2011b); (18) Moór et al. (2015a); (19) Donaldson et al., priv. comm.; (20) Cotten \& Song (2016); (21) Plavchan et al. (2009) (22) Cataldi et al. (2019) References for associations: (1) Chen et al. (2014); (2) Zuckerman \& Song (2012); (3) Riviere-Marichalar et al. (2013); (4) Donaldson et al. (2012); (5) Lieman-Sifry et al. (2016); (6) de Zeeuw et al. (1999);(7) Zuckerman (2019); (8) Desidera et al. (2015) References for selection criteria: (1) Donaldson et al. (2012); (2) Riviere-Marichalar et al. (2014); (3) Redfield et al. (2007) ; (4) Ertel et al. (2014); (5) Chen et al. (2014); (6) Nuñez et al. (2017); (7) Welsh \& Montgomery (2013); (8) Absil et al. (2013); (9) Pawellek et al. (2014); (10) Moór et al. (2011b); (11) Abt (2008); (12) Draper et al. (2016); (13) Redfield (2007); (14) Currie et al. (2017); (15) Roberge \& Weinberger (2008); (16) Welsh \& Montgomery (2015); (17) Marino et al. (2017); (18) Moór et al. (2017); (19) Lagrange-Henri et al. (1990b); (20) Donaldson et al., priv. comm.; (21) Lieman-Sifry et al. (2016); (22) Kiefer et al. (2014b); (23) Marino et al. (2016); (24) Chen et al. (2005) 
Table B.1. continued.

\begin{tabular}{|c|c|c|c|c|c|c|c|c|c|c|c|c|}
\hline HD & Other name & RA(2000.0) & $\operatorname{Dec}(2000.0)$ & Sp. type & $\begin{array}{r}\text { Distance } \\
(\mathrm{pc})\end{array}$ & $\begin{array}{l}V \\
\text { (mag) }\end{array}$ & $\begin{array}{l}B-V \\
(\mathrm{mag})\end{array}$ & $\begin{array}{r}V_{\text {rad }} \\
\left(\mathrm{km} \mathrm{s}^{-1}\right)\end{array}$ & $\begin{array}{r}\text { Age } \\
(\mathrm{Myr})\end{array}$ & $L_{\mathrm{IR}} / L_{*}$ & Assoc. & Sel.crit \\
\hline 95418 & $\beta \mathrm{UMa}$ & $11: 01: 50.48$ & $+56: 22: 56.7$ & A1IV & 24.45 & 2.37 & -0.02 & -13.1 & $320(1)$ & $1.4 \times 10^{-5}$ & UMA (1) & $2(9)$ \\
\hline 98058 & $\phi$ Leo & $11: 16: 39.70$ & $-03: 39: 05.8$ & A7IV & 56.47 & 4.47 & 0.198 & -3.0 & $921(8)$ & $<7.4 \times 10^{-5}(23)$ & & $6(11)$ \\
\hline 102647 & $\beta$ Leo & $11: 49: 03.58$ & $+14: 34: 19.4$ & $\mathrm{~A} 3 \mathrm{Va}$ & 11.00 & 2.13 & 0.09 & -0.2 & $45(1)$ & $2.2 \times 10^{-5}(4)$ & IC 2391 (1) & $4(8)$ \\
\hline 104731 & HR 4600 & $12: 03: 39.57$ & $-42: 26: 02.6$ & F5V & 24.73 & 5.15 & 0.41 & 38.5 & $360(6)$ & & CANE (1) & $4(4)$ \\
\hline 104860 & HIP 58876 & $12: 04: 33.73$ & $+66: 20: 11.7$ & F8 & 45.20 & 7.91 & 0.59 & -11.73 & $3140(1)$ & $3.0 \times 10^{-4}(6)$ & & $2(9)$ \\
\hline 105234 & EF Cha & $12: 07: 05.52$ & $-78: 44: 28.0$ & A9III/4 & 104.54 & 7.46 & 0.28 & 0.0 & $10(1)$ & $1.0 \times 10^{-3}(13)$ & & $2(5)$ \\
\hline 105850 & HR 4635 & $12: 11: 03.84$ & $-23: 36: 08.7$ & A1V & 58.81 & 5.47 & 0.05 & 11.0 & $45(9)$ & $6.2 \times 10^{-5}(6)$ & Tuc-Hor (2) & $2(5)$ \\
\hline 108767 & $\delta \mathrm{Crv}$ & $12: 29: 51.86$ & $-16: 30: 55.6$ & A0IV & 26.63 & 2.94 & -0.05 & 13.9 & $216(8)$ & $<4.4 \times 10^{-6}(4)$ & & $4(4)$ \\
\hline 109085 & $\eta \mathrm{Crv}$ & $12: 32: 04.23$ & $-16: 11: 45.6$ & $\mathrm{~F} 2 \mathrm{~V}$ & 18.28 & 4.31 & 0.38 & -2.8 & $1400(13)$ & $3.4 \times 10^{-4}(14)$ & & $3(17)$ \\
\hline 109573 & HR 4796 & $12: 36: 01.03$ & $-39: 52: 10.2$ & $\mathrm{~A} 0 \mathrm{~V}$ & 71.91 & 5.78 & 0.009 & 7.1 & $8(1)$ & $4.6 \times 10^{-3}(15)$ & TWA (1) & $2(5)$ \\
\hline 110058 & HIP 61782 & $12: 39: 46.20$ & $-49: 11: 55.5$ & $\mathrm{~A} 0 \mathrm{~V}$ & 129.98 & 7.97 & 0.15 & 5.0 & $15(14)$ & $1.4 \times 10^{-3}(6)$ & LCC (5) & $3(18)$ \\
\hline 110411 & $\rho$ Vir & $12: 41: 53.06$ & $+10: 14: 08.3$ & $\mathrm{~A} 3 \mathrm{~V}$ & 38.16 & 4.88 & 0.09 & -0.7 & $86(1)$ & $6.4 \times 10^{-5}$ & & $1(7)$ \\
\hline 118232 & $24 \mathrm{CVn}$ & $13: 34: 27.26$ & $+49: 00: 57.5$ & $\mathrm{~A} 4 \mathrm{~V}$ & 55.28 & 4.7 & 0.12 & -18.3 & $612(1)$ & $2.6 \times 10^{-5}(11)$ & & $6(15)$ \\
\hline 121191 & SAO 241295 & $13: 55: 18.86$ & $-53: 31: 43.0$ & A5IV/V & 132.11 & 8.16 & 0.24 & 12.0 & $16(14)$ & $4.7 \times 10^{-3}(16)$ & LCC/UCL (8) & $3(18)$ \\
\hline 121617 & SAO 224570 & $13: 57: 41.13$ & $-47: 00: 34.2$ & A1V & 116.87 & 7.29 & 0.07 & 7.8 & $16(14)$ & $4.8 \times 10^{-3}(17)$ & UCL (9) & $3(18)$ \\
\hline 125162 & $\lambda B \ddot{o} o$ & $14: 16: 23.02$ & $+46: 05: 17.9$ & A3 & 30.36 & 4.18 & 0.08 & 7.9 & $313(1)$ & $4.4 \times 10^{-5}$ & & $7(12)$ \\
\hline 131488 & SAO 225290 & $14: 55: 08.03$ & $-41: 07: 13.4$ & A1V & 154.62 & 8.0 & 0.09 & 5.8 & $16(14)$ & $5.5 \times 10^{-3}(16)$ & UCL (8) & $3(18)$ \\
\hline 131835 & HIP 73145 & $14: 56: 54.47$ & $-35: 41: 43.7$ & A2IV & 133.65 & 7.86 & 0.19 & 0.5 & $16(14)$ & $3.0 \times 10^{-3}(18)$ & UCL (5) & $3(18)$ \\
\hline 138629 & HR 5774 & $15: 31: 46.98$ & $+40: 53: 57.6$ & $\mathrm{~A} 5 \mathrm{~V}$ & 127.20 & 4.98 & 0.96 & -16.0 & - & & & $1(19)$ \\
\hline 139006 & $\alpha \mathrm{CrB}$ & $15: 34: 41.27$ & $+26: 42: 52.9$ & $\mathrm{~A} 0 \mathrm{~V}$ & 23.01 & 2.24 & -0.02 & 1.4 & $314(1)$ & $1.5 \times 10^{-5}(4)$ & UMA (1) & $2(9)$ \\
\hline 138813 & HIP 76310 & $15: 35: 16.11$ & $-25: 44: 03.0$ & $\mathrm{~A} 0 \mathrm{~V}$ & 137.41 & 7.3 & 0.07 & 4.4 & $10(14)$ & $9.0 \times 10^{-4}(6)$ & US (6) & $3(18)$ \\
\hline 142097 & HIP 77815 & $15: 53: 21.93$ & $-21: 58: 16.7$ & A5V & 140.92 & 8.39 & 0.41 & -0.1 & $11(16)$ & $<3.0 \times 10^{-4}(19)$ & US (6) & $5(20)$ \\
\hline 142315 & HIP 77911 & $15: 54: 41.60$ & $-22: 45: 58.5$ & B9V & 145.34 & 6.87 & 0.034 & -7.4 & $11(1)$ & $3.8 \times 10^{-4}(19)$ & US (6) & $5(20)$ \\
\hline 142705 & HIP 78099 & $15: 56: 47.85$ & $-23: 11: 02.7$ & $\mathrm{~A} 0 \mathrm{~V}$ & 144.33 & 7.74 & 0.18 & -6.5 & $11(16)$ & $<1.7 \times 10^{-4}(19)$ & US (6) & $5(20)$ \\
\hline 144587 & HIP 78996 & $16: 07: 29.93$ & $-23: 57: 02.4$ & A9V & 144.01 & 8.31 & 0.42 & 0.0 & $11(1)$ & $3.2 \times 10^{-4}(19)$ & US (6) & $5(20)$ \\
\hline 144981 & HIP 79156 & $16: 09: 20.89$ & $-19: 27: 25.9$ & $\mathrm{~A} 0 \mathrm{~V}$ & 150.59 & 8.04 & 0.18 & -1.3 & $11(1)$ & $1.2 \times 10^{-4}(19)$ & US (6) & $5(20)$ \\
\hline 145554 & HIP 79410 & $16: 12: 21.83$ & $-19: 34: 44.6$ & B9V & 136.83 & 7.64 & 0.13 & -9.4 & $11(1)$ & $1.4 \times 10^{-4}(19)$ & US (6) & $5(20)$ \\
\hline 145631 & HIP 79439 & $16: 12: 44.10$ & $-19: 30: 10.3$ & B9V & 140.72 & 7.6 & 0.13 & -9.5 & $11(1)$ & $6.3 \times 10^{-5}(19)$ & US (6) & $5(20)$ \\
\hline 145964 & HR 6051 & $16: 14: 28.88$ & $-21: 06: 27.5$ & B9V & 112.18 & 6.41 & 0.001 & -7.8 & $11(1)$ & $1.5 \times 10^{-5}(6)$ & US (6) & $1(7)$ \\
\hline 145689 & HIP 79797 & $16: 17: 05.41$ & $-67: 56: 28.6$ & $\mathrm{~A} 6 \mathrm{~V}$ & 55.55 & 5.95 & 0.148 & -9.0 & $40(1)$ & $4.9 \times 10^{-5}(6)$ & Argus (1) & $5(21)$ \\
\hline 146606 & HIP 79878 & $16: 18: 16.16$ & $-28: 02: 30.2$ & $\mathrm{~A} 0 \mathrm{~V}$ & 137.27 & 7.06 & -0.01 & 0.8 & $11(1)$ & $9.5 \times 10^{-5}(19)$ & US (6) & $5(20)$ \\
\hline 146624 & d Sco & $16: 18: 17.90$ & $-28: 36: 50.5$ & $\mathrm{~A} 0 \mathrm{~V}$ & 41.29 & 4.79 & 0.03 & -13.0 & $23(2)$ & $<5.0 \times 10^{-7}(2)$ & BPMG (3) & $5(2)$ \\
\hline 146897 & HIP 79977 & $16: 19: 29.24$ & $-21: 24: 13.3$ & $\mathrm{~F} 2 / 3 \mathrm{~V}$ & 131.50 & 9.11 & 0.47 & -1.1 & $15(16)$ & $5.6 \times 10^{-3}(6)$ & US (6) & $3(21)$ \\
\hline 147137 & HIP 80088 & $16: 20: 50.23$ & $-22: 35: 38.8$ & A9V & 143.92 & 9.03 & 0.39 & -0.8 & $11(1)$ & $5.7 \times 10^{-4}(19)$ & US (6) & $5(20)$ \\
\hline 147220 & HIP 80130 & $16: 21: 21.15$ & $-22: 06: 32.3$ & A9V & 158.88 & 8.59 & 0.45 & -1.0 & - & $<3.3 \times 10^{-4}(19)$ & US (6) & $5(20)$ \\
\hline 148283 & HR 6123 & $16: 25: 24.17$ & $+37: 23: 38.7$ & A5V & 77.45 & 5.54 & 0.17 & -1.3 & $854(8)$ & $<8.4 \times 10^{-6}(11)$ & & $6(11)$ \\
\hline 156623 & HIP 84881 & $17: 20: 50.62$ & $-45: 25: 15.0$ & $\mathrm{~A} 0 \mathrm{~V}$ & 111.75 & 7.26 & 0.09 & -0.2 & $16(14)$ & $7.8 \times 10^{-3}(20)$ & UCL (5) & $3(21)$ \\
\hline 157728 & $73 \mathrm{Her}$ & $17: 24: 06.59$ & $+22: 57: 37.0$ & A7V & 42.75 & 5.72 & 0.21 & -19.7 & $534(1)$ & $2.9 \times 10^{-4}(6)$ & & $2(5)$ \\
\hline 158352 & HR 6507 & $17: 28: 49.66$ & $+00: 19: 50.3$ & $\mathrm{~A} 8 \mathrm{Vp}$ & 63.46 & 5.41 & 0.23 & -36.1 & 890 (1) & $9.3 \times 10^{-5}(11)$ & & $2(5)$ \\
\hline 162003 & $\psi 1$ Dra A & $17: 41: 56.35$ & $+72: 08: 55.8$ & F5IV/V & 21.42 & 4.56 & 0.44 & -13.3 & $2326(8)$ & $<4.4 \times 10^{-6}(8)$ & & $4(6)$ \\
\hline 162917 & HR 6670 & $17: 53: 14.19$ & $+06: 06: 05.1$ & F4IV/V & 30.80 & 5.77 & 0.4 & -29.1 & $1210(1)$ & $6.5 \times 10^{-5}(6)$ & & $2(5)$ \\
\hline 164249 & HIP 88399 & $18: 03: 03.41$ & $-51: 38: 56.4$ & $\mathrm{~F} 6 \mathrm{~V}+\mathrm{M} 2 \mathrm{~V}$ & 49.61 & 7.01 & 0.456 & -0.4 & $23(2)$ & $8.4 \times 10^{-4}(2)$ & BPMG (3) & $5(2)$ \\
\hline 168646 & HR 6864 & $18: 22: 00.14$ & $-28: 25: 47.9$ & A3III & 191.33 & 6.15 & 0.242 & -11.5 & $662(3)$ & & & $6(11)$ \\
\hline 172555 & HR 7012 & $18: 45: 26.90$ & $-64: 52: 16.5$ & A7V & 28.55 & 4.78 & 0.191 & 2.0 & $23(2)$ & $7.1 \times 10^{-4}(4)$ & BPMG (3) & $1(22)$ \\
\hline 173667 & $110 \mathrm{Her}$ & $18: 45: 39.73$ & $+20: 32: 46.7$ & F6V & 19.21 & 4.19 & 0.46 & 23.05 & $2687(8)$ & $7.0 \times 10^{-7}(9)$ & & $4(8)$ \\
\hline 177724 & $\zeta$ Aql & $19: 05: 24.61$ & $+13: 51: 48.5$ & A0IV/Vn & 25.46 & 2.99 & 0.01 & -25.0 & $356(1)$ & $<1.6 \times 10^{-6}$ & & $4(8)$ \\
\hline 181296 & $\eta \mathrm{Tel}$ & $19: 22: 51.21$ & $-54: 25: 26.1$ & $\mathrm{~A} 0 \mathrm{~V}+\mathrm{M} 7 \mathrm{~V}$ & 48.22 & 5.02 & 0.015 & 13.0 & $23(2)$ & $2.4 \times 10^{-4}(6)$ & BPMG (3) & $3(2)$ \\
\hline 181327 & HIP 95270 & $19: 22: 58.94$ & $-54: 32: 17.0$ & F5/F6V & 48.21 & 7.04 & 0.46 & 0.2 & $23(2)$ & $2.0 \times 10^{-3}(6)$ & BPMG (3) & $3(23)$ \\
\hline 182640 & $\delta$ Aql & $19: 25: 29.90$ & $+03: 06: 53.2$ & F1IV & 15.53 & 3.36 & 0.32 & -34.0 & $1385(8)$ & & & $4(6)$ \\
\hline 182919 & $5 \mathrm{Vul}$ & $19: 26: 13.25$ & $+20: 05: 51.8$ & $\mathrm{~A} 0 \mathrm{~V}$ & 71.98 & 5.59 & 0.002 & -20.9 & $198(1)$ & $3.4 \times 10^{-5}(6)$ & & $1(7)$ \\
\hline 183324 & c Aql & $19: 29: 00.99$ & $+01: 57: 01.6$ & A0IV & 60.68 & 5.79 & 0.083 & 12.0 & $140(1)$ & $1.8 \times 10^{-5}$ & & $1(7)$ \\
\hline 187642 & $\alpha \mathrm{Aql}$ & $19: 50: 47.00$ & $+08: 52: 06.0$ & A7V & 5.13 & 0.76 & 0.22 & -26.6 & $991(8)$ & $<4.4 \times 10^{-6}(4)$ & & $4(8)$ \\
\hline 188228 & $\epsilon \mathrm{Pav}$ & $20: 00: 35.56$ & $-72: 54: 37.8$ & $\mathrm{~A} 0 \mathrm{~V}$ & 32.22 & 3.95 & -0.006 & -6.7 & $40(1)$ & $4.4 \times 10^{-6}(4)$ & Argus (7) & $2(9)$ \\
\hline 192518 & HR 7731 & $20: 14: 14.53$ & $+28: 41: 41.3$ & A5IV & 93.37 & 5.2 & 0.17 & 7.0 & $607(8)$ & & & $6(11)$ \\
\hline 196724 & $29 \mathrm{Vul}$ & $20: 38: 31.34$ & $+21: 12: 04.3$ & A0 & 64.00 & 4.82 & -0.02 & -17.1 & $224(8)$ & $<2.4 \times 10^{-6}(11)$ & & $6(15)$ \\
\hline 198160 & HR 7959 & $20: 51: 38.47$ & $-62: 25: 45.6$ & A2III & 74.00 & 6.21 & 0.14 & -16.0 & $735(8)$ & $2.0 \times 10^{-5}(5)$ & & $7(12)$ \\
\hline 198161 & HR 7960 & $20: 51: 38.76$ & $-62: 25: 44.9$ & A3III & 73.90 & 6.56 & 0.18 & -10.0 & $735(8)$ & & & $7(12)$ \\
\hline 199143 & HIP 103311 & $20: 55: 47.67$ & $-17: 06: 51.0$ & F8V & 45.66 & 7.32 & 0.52 & -4.5 & $23(2)$ & $<2.6 \times 10^{-6}(2)$ & BPMG & $5(2)$ \\
\hline 203280 & $\alpha$ Cep & $21: 18: 34.77$ & $+62: 35: 08.1$ & $\mathrm{~A} 8 \mathrm{~V}$ & 15.04 & 2.46 & 0.22 & -15.8 & $987(8)$ & $<2.1 \times 10^{-6}(24)$ & & $4(8)$ \\
\hline 202917 & HIP 105388 & $21: 20: 49.96$ & $-53: 02: 03.1$ & G7V & 46.85 & 8.67 & 0.65 & -0.9 & $45(9)$ & $2.5 \times 10^{-4}(1)$ & Tuc-Hor (4) & $5(1)$ \\
\hline 210302 & $\tau$ PsA & $22: 10: 08.78$ & $-32: 32: 54.3$ & F6V & 18.35 & 4.92 & 0.48 & -16.25 & $3530(7)$ & $<4.4 \times 10^{-6}(8)$ & & $4(4)$ \\
\hline 210418 & $\theta$ Peg & $22: 10: 11.99$ & $+06: 11: 52.3$ & A1V & 28.30 & 3.55 & 0.07 & -7.9 & $545(8)$ & $<4.4 \times 10^{-6}$ & & $4(6)$ \\
\hline 211336 & $\epsilon$ Cep & $22: 15: 02.20$ & $+57: 02: 36.9$ & F0IV & 26.30 & 4.19 & 0.28 & -4.7 & $1063(1)$ & $1.9 \times 10^{-5}(6)$ & & $4(8)$ \\
\hline 213617 & 39 Peg & $22: 32: 35.48$ & $+20: 13: 48.1$ & F1V & 53.58 & 6.44 & 0.33 & -18.9 & $930(1)$ & $6.5 \times 10^{-5}(6)$ & & $2(5)$ \\
\hline 217782 & 2 And & $23: 02: 36.38$ & $+42: 45: 28.1$ & $\mathrm{~A} 3 \mathrm{~V}$ & 129.20 & 5.1 & 0.08 & 2.1 & $478(3)$ & $<9.6 \times 10^{-6}(11)$ & & $1(7)$ \\
\hline 221756 & 15 And & $23: 34: 37.54$ & $+40: 14: 11.2$ & A1V & 77.28 & 5.56 & 0.089 & 13.1 & $613(8)$ & $1.5 \times 10^{-5}$ & & $7(12)$ \\
\hline 222368 & $\iota$ Psc & $23: 39: 57.04$ & $+05: 37: 34.6$ & F7V & 13.71 & 4.12 & 0.5 & 5.67 & 3287 (3) & $1.1 \times 10^{-6}(8)$ & & $4(6)$ \\
\hline 224392 & $\eta$ Tuc & $23: 57: 35.08$ & $-64: 17: 53.6$ & A1V & 47.07 & 5.0 & 0.056 & 32.5 & $45(9)$ & $<1.6 \times 10^{-5}(1)$ & Tuc-Hor (4) & $5(1)$ \\
\hline
\end{tabular}


Table B.2. Stellar parameters estimated according to Sect. 3.2.

\begin{tabular}{|c|c|c|c|c|c|c|c|c|}
\hline HD & $\mathrm{RA}(2000.0)$ & $\operatorname{Dec}(2000.0)$ & Sp. type & $\begin{array}{c}v_{\mathrm{rad}} \\
\left(\mathrm{km} \mathrm{s}^{-1}\right)\end{array}$ & $\begin{array}{l}T_{\text {eff }} \\
(\mathrm{K})\end{array}$ & $\begin{array}{l}\log g \\
{[\mathrm{cgs}]}\end{array}$ & {$[M / H]$} & $\begin{array}{c}v \sin i \\
\left(\mathrm{~km} \mathrm{~s}^{-1}\right)\end{array}$ \\
\hline 105 & $00: 05: 52.54$ & $-41: 45: 11.0$ & $\mathrm{G} 0 \mathrm{~V}$ & $+1.6 \pm 0.4$ & $6000 \pm 60$ & $4.38 \pm 0.14$ & -0.03 & 14.6 \\
\hline 203 & $00: 06: 50.09$ & $-23: 06: 27.1$ & F3V & $+7.1 \pm 1.7$ & $6850 \pm 200$ & $4.25 \pm 0.30$ & 0.00 & 170 \\
\hline 256 & $00: 07: 18.27$ & $-17: 23: 13.2$ & $\mathrm{~A} 2 \mathrm{IV} / \mathrm{V}$ & $-2.2 \pm 0.3$ & $8690 \pm 50$ & $3.48 \pm 0.20$ & 0.00 & 270 \\
\hline 1466 & $00: 18: 26.12$ & $-63: 28: 39.0$ & F8V & $+6.4 \pm 0.4$ & $6270 \pm 60$ & $4.45 \pm 0.15$ & -0.01 & 18 \\
\hline 2262 & $00: 26: 12.20$ & $-43: 40: 47.4$ & A5IV & $+11.3 \pm 3.7$ & $8110 \pm 30$ & $4.01 \pm 0.13$ & 0.00 & 200 \\
\hline 2884 & $00: 31: 32.67$ & $-62: 57: 29.6$ & B9V & $+8.3 \pm 1.6$ & $11620 \pm 30$ & $4.45 \pm 0.10$ & -0.05 & 135 \\
\hline 2885 & $00: 31: 33.48$ & $-62: 57: 56.0$ & $\mathrm{~A} 2 \mathrm{~V}$ & $+13.5 \pm 3.9$ & $9630 \pm 30$ & $4.27 \pm 0.10$ & +0.20 & 40 \\
\hline 3003 & $00: 32: 43.91$ & $-63: 01: 53.4$ & $\mathrm{~A} 0 \mathrm{~V}$ & $+8.2 \pm 2.3$ & $9490 \pm 50$ & $4.35 \pm 0.14$ & 0.00 & 95 \\
\hline 5267 & $00: 54: 35.23$ & $+19: 11: 18.3$ & A1V & $+9.5 \pm 1.8$ & $10450 \pm 50$ & $4.16 \pm 0.10$ & 0.00 & 144 \\
\hline 5448 & $00: 56: 45.21$ & $+38: 29: 57.6$ & A5V & $+6.4 \pm 0.6$ & $8590 \pm 70$ & $3.18 \pm 0.10$ & +0.20 & 65 \\
\hline 7788 & $01: 15: 46.16$ & $-68: 52: 33.3$ & $\mathrm{~F} 6 \mathrm{~V}+\mathrm{K} 1 \mathrm{~V}$ & $+12.4 \pm 3.3$ & $6550 \pm 200$ & $4.30 \pm 0.10$ & 0.00 & 70 \\
\hline 9672 & $01: 34: 37.78$ & $-15: 40: 34.9$ & A1V & $+10.9 \pm 2.5$ & $9120 \pm 50$ & $4.32 \pm 0.16$ & 0.00 & 186 \\
\hline 10700 & $01: 44: 04.08$ & $-15: 56: 14.9$ & $\mathrm{G} 8.5 \mathrm{~V}$ & $-16.8 \pm 0.1$ & $5330 \pm 10$ & $4.55 \pm 0.10$ & -0.51 & 1.6 \\
\hline 12039 & $01: 57: 48.98$ & $-21: 54: 05.3$ & G4V & $+5.8 \pm 0.4$ & $5700 \pm 40$ & $4.31 \pm 0.10$ & -0.06 & 15.9 \\
\hline 14055 & $02: 17: 18.87$ & $+33: 50: 49.9$ & A1V & $+7.3 \pm 0.2$ & $10040 \pm 50$ & $4.34 \pm 0.10$ & 0.00 & 254 \\
\hline 14412 & $02: 18: 58.50$ & $-25: 56: 44.5$ & G8V & $+7.3 \pm 0.2$ & $5310 \pm 20$ & $4.55 \pm 0.10$ & -0.53 & 0.90 \\
\hline 15115 & $02: 26: 16.24$ & $+06: 17: 33.2$ & $\mathrm{~F} 2 \mathrm{~V}$ & $+6.0 \pm 2.7$ & $6750 \pm 200$ & $4.25 \pm 0.30$ & 0.00 & 89.8 \\
\hline 15257 & $02: 28: 09.98$ & $+29: 40: 09.6$ & FOIII & $-20.6 \pm 0.2$ & $7100 \pm 200$ & $3.50 \pm 0.30$ & 0.00 & 65 \\
\hline 16978 & $02: 39: 35.36$ & $-68: 16: 01.0$ & B9III & $-24.8 \pm 0.8$ & $11330 \pm 100$ & $4.43 \pm 0.11$ & 0.00 & 96 \\
\hline 21688 & $03: 29: 36.03$ & $-12: 40: 29.1$ & A5III/IV & $+15.2 \pm 0.1$ & $7880 \pm 90$ & $4.52 \pm 0.30$ & 0.00 & 180 \\
\hline 21620 & $03: 31: 29.34$ & $+49: 12: 35.2$ & A0V & $-13.9 \pm 0.3$ & $9650 \pm 50$ & $3.90 \pm 0.10$ & 0.00 & 260 \\
\hline 21997 & $03: 31: 53.65$ & $-25: 36: 50.9$ & $\mathrm{~A} 3 \mathrm{IV} / \mathrm{V}$ & $+19.0 \pm 1.5$ & $8520 \pm 140$ & $4.27 \pm 0.31$ & 0.00 & 60 \\
\hline 22484 & $03: 36: 52.38$ & $+00: 24: 06.0$ & F8V & $+27.9 \pm 0.1$ & $5960 \pm 30$ & $3.93 \pm 0.10$ & -0.09 & 4 \\
\hline 27290 & $04: 16: 01.59$ & $-51: 29: 11.9$ & F1V & $+26.7 \pm 1.6$ & $7300 \pm 200$ & $4.25 \pm 0.25$ & 0.00 & 60 \\
\hline 28355 & $04: 28: 50.16$ & $+13: 02: 51.4$ & $\mathrm{~A} 7 \mathrm{~V}$ & $+39.8 \pm 0.3$ & $8730 \pm 50$ & $3.53 \pm 0.30$ & +0.50 & 105 \\
\hline 29391 & $04: 37: 36.13$ & $-02: 28: 24.8$ & FOIV & $+20.4 \pm 3.8$ & $7200 \pm 100$ & $4.25 \pm 0.25$ & 0.00 & 84 \\
\hline 30051 & $04: 43: 17.20$ & $-23: 37: 42.0$ & $\mathrm{~F} 2 \mathrm{~V}$ & $+15.9 \pm 5.6$ & $6900 \pm 200$ & $4.25 \pm 0.25$ & 0.00 & 50 \\
\hline 31295 & $04: 54: 53.73$ & $+10: 09: 03.0$ & $\mathrm{~A} 3 \mathrm{~V}$ & $+16.0 \pm 0.2$ & $9130 \pm 80$ & $4.25 \pm 0.26$ & -1.00 & 120 \\
\hline 32297 & $05: 02: 27.44$ & $+07: 27: 39.7$ & $\mathrm{~A} 0 \mathrm{~V}$ & $+21.0 \pm 0.9$ & $7980 \pm 100$ & $3.77 \pm 0.30$ & -0.50 & 90 \\
\hline 35850 & $05: 27: 04.76$ & $-11: 54: 03.5$ & F8V & $+21.1 \pm 3.3$ & $6400 \pm 200$ & $4.25 \pm 0.25$ & 0.00 & 50 \\
\hline 36546 & $05: 33: 30.76$ & $+24: 37: 43.7$ & B8V & $+14.7 \pm 0.6$ & $9510 \pm 50$ & $4.44 \pm 0.10$ & -1.00 & 150 \\
\hline 37286 & $05: 36: 10.30$ & $-28: 42: 28.8$ & A2IV & $+24.9 \pm 2.9$ & $8640 \pm 50$ & $4.11 \pm 0.12$ & 0.00 & 70 \\
\hline 37306 & $05: 37: 08.77$ & $-11: 46: 31.9$ & $\mathrm{~A} 2 \mathrm{~V}$ & $+25.1 \pm 1.3$ & $9600 \pm 50$ & $4.48 \pm 0.10$ & 0.00 & 144 \\
\hline 38206 & $05: 43: 21.67$ & $-18: 33: 26.9$ & $\mathrm{~A} 0 \mathrm{~V}$ & $+26.6 \pm 2.3$ & $10480 \pm 100$ & $4.50 \pm 0.10$ & 0.00 & 35 \\
\hline 39182 & $05: 52: 39.67$ & $+39: 34: 28.9$ & $\mathrm{~A} 2 \mathrm{~V}$ & $-22.9 \pm 1.0$ & $9020 \pm 80$ & $3.42 \pm 0.30$ & +0.20 & 238 \\
\hline 39283 & $05: 54: 50.78$ & $+55: 42: 25.0$ & $\mathrm{~A} 2 \mathrm{~V}$ & $-18.3 \pm 0.6$ & $9140 \pm 40$ & $3.91 \pm 0.15$ & -0.25 & 68 \\
\hline 40136 & $05: 56: 24.29$ & $-14: 10: 03.7$ & $\mathrm{~F} 2 \mathrm{~V}$ & $+1.6 \pm 0.8$ & $7150 \pm 200$ & $4.25 \pm 0.25$ & 0.00 & 18 \\
\hline 42111 & $06: 08: 57.90$ & $+02: 29: 58.9$ & $\mathrm{~A} 3 \mathrm{~V}$ & $+27.5 \pm 1.9$ & $9380 \pm 40$ & $3.48 \pm 0.10$ & 0.00 & 252 \\
\hline 53842 & $06: 46: 13.54$ & $-83: 59: 29.5$ & F5V & $+17.0 \pm 0.5$ & $6500 \pm 200$ & $4.25 \pm 0.25$ & 0.00 & 50 \\
\hline 50241 & $06: 48: 11.46$ & $-61: 56: 29.0$ & $\mathrm{~A} 8 \mathrm{~V}$ & $+13.0 \pm 2.9$ & $7580 \pm 90$ & $3.52 \pm 0.30$ & -0.25 & 206 \\
\hline 50571 & $06: 50: 01.01$ & $-60: 14: 56.9$ & F5V & $+26.2 \pm 3.1$ & $6450 \pm 100$ & $4.25 \pm 0.25$ & 0.00 & 50 \\
\hline 56537 & $07: 18: 05.58$ & $+16: 32: 25.4$ & A4IV & $-9.6 \pm 2.7$ & $8190 \pm 100$ & $4.10 \pm 0.39$ & 0.00 & 154 \\
\hline 64145 & $07: 53: 29.81$ & $+26: 45: 56.8$ & A5IV & $+4.2 \pm 3.2$ & $8320 \pm 50$ & $3.48 \pm 0.10$ & 0.00 & 165 \\
\hline 71043 & $08: 22: 55.16$ & $-52: 07: 25.4$ & $\mathrm{~A} 0 \mathrm{~V}$ & $+24.1 \pm 2.2$ & $10280 \pm 50$ & $4.51 \pm 0.10$ & 0.00 & 224 \\
\hline 71722 & $08: 26: 25.21$ & $-52: 48: 27.0$ & $\mathrm{~A} 0 \mathrm{~V}$ & $+31.0 \pm 2.6$ & $8870 \pm 50$ & $4.19 \pm 0.12$ & -1.00 & 220 \\
\hline 74873 & $08: 46: 56.02$ & $+12: 06: 35.8$ & A1VP & $+23.0 \pm 0.2$ & $8800 \pm 70$ & $4.02 \pm 0.37$ & -1.00 & 115 \\
\hline 77190 & $09: 01: 48.84$ & $+27: 54: 09.3$ & A8Vn & $+14.5 \pm 3.1$ & $8010 \pm 120$ & $3.41 \pm 0.30$ & 0.00 & 185 \\
\hline 80007 & $09: 13: 11.98$ & $-69: 43: 01.9$ & A1III & $+0.8 \pm 1.8$ & $9350 \pm 40$ & $3.47 \pm 0.10$ & 0.00 & 135 \\
\hline 85905 & $09: 54: 31.82$ & $-22: 29: 14.9$ & A1IV & $+9.9 \pm 2.5$ & $9040 \pm 40$ & $3.50 \pm 0.10$ & 0.00 & 285 \\
\hline 95418 & $11: 01: 50.48$ & $+56: 22: 56.7$ & A1IV & $-12.2 \pm 0.6$ & $9700 \pm 40$ & $4.01 \pm 0.19$ & 0.00 & 46 \\
\hline 98058 & $11: 16: 39.70$ & $-03: 39: 05.8$ & A7IV & $-0.3 \pm 0.8$ & $7500 \pm 50$ & $3.75 \pm 0.32$ & 0.00 & 230 \\
\hline 102647 & $11: 49: 03.58$ & $+14: 34: 19.4$ & A3Va & $-0.4 \pm 3.5$ & $8580 \pm 50$ & $4.14 \pm 0.32$ & 0.00 & 128 \\
\hline 104731 & $12: 03: 39.57$ & $-42: 26: 02.6$ & F5V & $+38.5 \pm 0.4$ & $6510 \pm 70$ & $3.87 \pm 0.10$ & -0.25 & 7 \\
\hline 104860 & $12: 04: 33.73$ & $+66: 20: 11.7$ & F8 & $-11.9 \pm 0.4$ & $6060 \pm 70$ & $4.48 \pm 0.14$ & -0.01 & 15 \\
\hline 105234 & $12: 07: 05.52$ & $-78: 44: 28.0$ & A9III/4 & $+15.3 \pm 1.9$ & $8590 \pm 50$ & $4.40 \pm 0.10$ & +0.50 & 85 \\
\hline 105850 & $12: 11: 03.84$ & $-23: 36: 08.7$ & A1V & $+15.0 \pm 3.6$ & $9160 \pm 50$ & $4.21 \pm 0.13$ & 0.00 & 128 \\
\hline 108767 & $12: 29: 51.86$ & $-16: 30: 55.6$ & A0IV & $+8.5 \pm 1.1$ & $10850 \pm 50$ & $4.35 \pm 0.10$ & 0.00 & 236 \\
\hline 109085 & $12: 32: 04.23$ & $-16: 11: 45.6$ & $\mathrm{~F} 2 \mathrm{~V}$ & $+0.0 \pm 0.5$ & $6950 \pm 100$ & $4.20 \pm 0.25$ & 0.00 & 60 \\
\hline
\end{tabular}


Table B.2. continued.

\begin{tabular}{|c|c|c|c|c|c|c|c|c|}
\hline HD & RA(2000.0) & $\operatorname{Dec}(2000.0)$ & Sp. type & $\begin{array}{c}v_{\mathrm{rad}} \\
\left(\mathrm{km} \mathrm{s}^{-1}\right)\end{array}$ & $\begin{array}{l}T_{\text {eff }} \\
(\mathrm{K})\end{array}$ & $\begin{array}{l}\log g \\
\text { [cgs] }\end{array}$ & {$[M / H]$} & $\begin{array}{c}v \sin i \\
\left(\mathrm{~km} \mathrm{~s}^{-1}\right)\end{array}$ \\
\hline 109573 & $12: 36: 01.03$ & $-39: 52: 10.2$ & $\mathrm{~A} 0 \mathrm{~V}$ & $+7.5 \pm 2.0$ & $10060 \pm 50$ & $4.44 \pm 0.10$ & -0.50 & 150 \\
\hline 110058 & $12: 39: 46.20$ & $-49: 11: 55.5$ & $\mathrm{~A} 0 \mathrm{~V}$ & $10.6 \pm 2.6$ & $9000 \pm 50$ & $4.13 \pm 0.15$ & -0.50 & 150 \\
\hline 110411 & $12: 41: 53.06$ & $+10: 14: 08.3$ & $\mathrm{~A} 3 \mathrm{~V}$ & $-7.9 \pm 1.1$ & $9240 \pm 50$ & $4.29 \pm 0.10$ & -1.00 & 154 \\
\hline 118232 & $13: 34: 27.26$ & $+49: 00: 57.5$ & $\mathrm{~A} 4 \mathrm{~V}$ & $-16.9 \pm 0.4$ & $8130 \pm 40$ & $3.49 \pm 0.10$ & 0.00 & 160 \\
\hline 121191 & $13: 55: 18.86$ & $-53: 31: 43.0$ & $\mathrm{~A} 5 \mathrm{IV} / \mathrm{V}$ & $21.5 \pm 1.0$ & $7970 \pm 90$ & $4.38 \pm 0.10$ & 0.00 & 65 \\
\hline 121617 & $13: 57: 41.13$ & $-47: 00: 34.2$ & A1V & $+7.0 \pm 3.7$ & $9160 \pm 70$ & $4.13 \pm 0.36$ & 0.00 & 90 \\
\hline 125162 & $14: 16: 23.02$ & $+46: 05: 17.9$ & A3 & $-10.1 \pm 1.5$ & $8660 \pm 50$ & $3.96 \pm 0.23$ & -2.00 & 100 \\
\hline 131488 & $14: 55: 08.03$ & $-41: 07: 13.4$ & A1V & $+4.8 \pm 0.2$ & $9130 \pm 60$ & $4.18 \pm 0.22$ & 0.00 & 120 \\
\hline 131835 & $14: 56: 54.47$ & $-35: 41: 43.7$ & A2IV & $+2.7 \pm 2.5$ & $8610 \pm 40$ & $4.24 \pm 0.18$ & 0.00 & 105 \\
\hline 138629 & $15: 31: 46.98$ & $+40: 53: 57.6$ & A5V & $-18.7 \pm 1.1$ & $8680 \pm 40$ & $3.54 \pm 0.10$ & +0.20 & 190 \\
\hline 139006 & $15: 34: 41.27$ & $+26: 42: 52.9$ & $\mathrm{~A} 0 \mathrm{~V}$ & $+9.9 \pm 01$ & $9780 \pm 40$ & $3.91 \pm 0.10$ & 0.00 & 130 \\
\hline 138813 & $15: 35: 16.11$ & $-25: 44: 03.0$ & $\mathrm{~A} 0 \mathrm{~V}$ & $+0.3 \pm 2.1$ & $9620 \pm 50$ & $4.30 \pm 0.11$ & 0.00 & 130 \\
\hline 142097 & $15: 53: 21.93$ & $-21: 58: 16.7$ & A5V & $-7.0 \pm 2.0$ & $8290 \pm 70$ & $3.67 \pm 0.10$ & +0.20 & 45 \\
\hline 142315 & $15: 54: 41.60$ & $-22: 45: 58.5$ & B9V & $-4.0 \pm 2.4$ & $12500 \pm 250$ & $4.50 \pm 0.25$ & 0.00 & 275 \\
\hline 142705 & $15: 56: 47.85$ & $-23: 11: 02.7$ & $\mathrm{~A} 0 \mathrm{~V}$ & $-5.6 \pm 3.0$ & $9300 \pm 50$ & $3.97 \pm 0.11$ & 0.00 & 300 \\
\hline 144587 & $16: 07: 29.93$ & $-23: 57: 02.4$ & A9V & $-10.7 \pm 4.7$ & $7390 \pm 250$ & $4.50 \pm 0.30$ & 0.00 & 112 \\
\hline 144981 & $16: 09: 20.89$ & $-19: 27: 25.9$ & $\mathrm{~A} 0 \mathrm{~V}$ & $-5.6 \pm 4.3$ & $9950 \pm 100$ & $4.32 \pm 0.22$ & 0.00 & 215 \\
\hline 145554 & $16: 12: 21.83$ & $-19: 34: 44.6$ & B9V & $-7.8 \pm 3.9$ & $12030 \pm 40$ & $4.44 \pm 0.10$ & 0.00 & 260 \\
\hline 145631 & $16: 12: 44.10$ & $-19: 30: 10.3$ & B9V & $-5.7 \pm 2.3$ & $11410 \pm 80$ & $4.36 \pm 0.10$ & 0.00 & 287 \\
\hline 145964 & $16: 14: 28.88$ & $-21: 06: 27.5$ & B9V & $-6.8 \pm 3.1$ & $12080 \pm 90$ & $4.47 \pm 0.13$ & 0.00 & 306 \\
\hline 145689 & $16: 17: 05.41$ & $-67: 56: 28.6$ & $\mathrm{~A} 6 \mathrm{~V}$ & $-7.1 \pm 1.7$ & $8340 \pm 50$ & $4.04 \pm 0.10$ & 0.00 & 105 \\
\hline 146606 & $16: 18: 16.16$ & $-28: 02: 30.2$ & $\mathrm{~A} 0 \mathrm{~V}$ & $-2.3 \pm 1.3$ & $10540 \pm 50$ & $4.39 \pm 0.10$ & 0.00 & 165 \\
\hline 146624 & $16: 18: 17.90$ & $-28: 36: 50.5$ & A0V & $-14.0 \pm 1.9$ & $9880 \pm 40$ & $4.50 \pm 0.10$ & 0.00 & 34 \\
\hline 146897 & $16: 19: 29.24$ & $-21: 24: 13.3$ & $\mathrm{~F} 2 / 3 \mathrm{~V}$ & $-3.1 \pm 0.9$ & $6700 \pm 200$ & $4.30 \pm 0.30$ & 0.00 & 55 \\
\hline 147137 & $16: 20: 50.23$ & $-22: 35: 38.8$ & A9V & $-6.6 \pm 1.9$ & $7670 \pm 40$ & $4.49 \pm 0.10$ & 0.00 & 82 \\
\hline 147220 & $16: 21: 21.15$ & $-22: 06: 32.3$ & A9V & $-49.3 \pm 2.0$ & $7560 \pm 80$ & $4.12 \pm 0.10$ & 0.00 & 80 \\
\hline 148283 & $16: 25: 24.17$ & $+37: 23: 38.7$ & $\mathrm{~A} 5 \mathrm{~V}$ & $-0.5 \pm 0.2$ & $8380 \pm 40$ & $4.46 \pm 0.30$ & 0.00 & 240 \\
\hline 156623 & $17: 20: 50.62$ & $-45: 25: 15.0$ & $\mathrm{~A} 0 \mathrm{~V}$ & $-0.2 \pm 2.1$ & $9430 \pm 80$ & $4.48 \pm 0.20$ & -0.30 & 75 \\
\hline 157728 & $17: 24: 06.59$ & $+22: 57: 37.0$ & A7V & $-21.2 \pm 0.3$ & $7690 \pm 46$ & $3.98 \pm 0.10$ & 0.00 & 60 \\
\hline 158352 & $17: 28: 49.66$ & $+00: 19: 50.3$ & $\mathrm{~A} 8 \mathrm{Vp}$ & $-33.9 \pm 0.6$ & $7470 \pm 140$ & $3.98 \pm 0.21$ & 0.00 & 150 \\
\hline 162003 & $17: 41: 56.35$ & $+72: 08: 55.8$ & F5IV/V & $-13.3 \pm 0.2$ & $6370 \pm 50$ & $3.62 \pm 0.10$ & -0.15 & 13 \\
\hline 162917 & $17: 53: 14.19$ & $+06: 06: 05.1$ & F4IV/V & $-29.1 \pm 0.9$ & $6620 \pm 220$ & $4.28 \pm 0.14$ & 0.00 & 28.4 \\
\hline 164249 & $18: 03: 03.41$ & $-51: 38: 56.4$ & $\mathrm{~F} 6 \mathrm{~V}+\mathrm{M} 2 \mathrm{~V}$ & $-0.4 \pm 0.5$ & $6570 \pm 90$ & $4.03 \pm 0.13$ & +0.02 & 21 \\
\hline 168646 & $18: 22: 00.14$ & $-28: 25: 47.9$ & A3III & $-10.8 \pm 2.6$ & $8750 \pm 100$ & $3.00 \pm 0.30$ & 0.00 & 260 \\
\hline 172555 & $18: 45: 26.90$ & $-64: 52: 16.5$ & A7V & $+1.2 \pm 2.9$ & $7994 \pm 60$ & $4.23 \pm 0.10$ & 0.00 & 107 \\
\hline 173667 & $18: 45: 39.73$ & $+20: 32: 46.7$ & F6V & $22.7 \pm 0.1$ & $6380 \pm 80$ & $3.67 \pm 0.12$ & -0.10 & 18 \\
\hline 177724 & $19: 05: 24.61$ & $+13: 51: 48.5$ & A0IV/Vn & $-27.3 \pm 2.5$ & $10260 \pm 50$ & $4.26 \pm 0.10$ & -0.15 & 290 \\
\hline 181296 & $19: 22: 51.21$ & $-54: 25: 26.1$ & $\mathrm{~A} 0 \mathrm{~V}+\mathrm{M} 7 \mathrm{~V}$ & $-5.0 \pm 1.5$ & $10500 \pm 170$ & $4.57 \pm 0.30$ & 0.00 & 230 \\
\hline 181327 & $19: 22: 58.94$ & $-54: 32: 17.0$ & F5/F6V & $+0.1 \pm 0.4$ & $6360 \pm 60$ & $4.09 \pm 0.10$ & -0.05 & 28 \\
\hline 182640 & $19: 25: 29.90$ & $+03: 06: 53.2$ & F1IV & $-37.2 \pm 1.8$ & $7000 \pm 200$ & $4.25 \pm 0.30$ & 0.00 & 91 \\
\hline 182919 & $19: 26: 13.25$ & $+20: 05: 51.8$ & $\mathrm{~A} 0 \mathrm{~V}$ & $-24.3 \pm 1.4$ & $10460 \pm 80$ & $4.47 \pm 0.10$ & 0.00 & 154 \\
\hline 183324 & $19: 29: 00.99$ & $+01: 57: 01.6$ & A0IV & $17.4 \pm 0.4$ & $9830 \pm 40$ & $4.50 \pm 0.10$ & -1.00 & 100 \\
\hline 187642 & $19: 50: 47.00$ & $+08: 52: 06.0$ & A7V & $-27.2 \pm 0.4$ & $7930 \pm 40$ & $4.21 \pm 0.10$ & 0.00 & 205 \\
\hline 188228 & $20: 00: 35.56$ & $-72: 54: 37.8$ & $\mathrm{~A} 0 \mathrm{~V}$ & $-6.0 \pm 3.5$ & $10620 \pm 50$ & $4.47 \pm 0.10$ & 0.00 & 85 \\
\hline 192518 & $20: 14: 14.53$ & $+28: 41: 41.3$ & A5IV & $+8.1 \pm 0.3$ & $8020 \pm 40$ & $4.61 \pm 0.10$ & 0.00 & 220 \\
\hline 196724 & $20: 38: 31.34$ & $+21: 12: 04.3$ & A0 & $-10.8 \pm 2.4$ & $10820 \pm 80$ & $4.35 \pm 0.12$ & 0.00 & 52 \\
\hline 198160 & $20: 51: 38.47$ & $-62: 25: 45.6$ & A2III & $-16.2 \pm 1.9$ & $8300 \pm 40$ & $4.15 \pm 0.30$ & -0.50 & 200 \\
\hline 198161 & $20: 51: 38.76$ & $-62: 25: 44.9$ & A3III & $-13.5 \pm 3.6$ & $8160 \pm 40$ & $3.79 \pm 0.30$ & -0.50 & 180 \\
\hline 199143 & $20: 55: 47.67$ & $-17: 06: 51.0$ & F8V & $-4.5 \pm 2.1$ & $6200 \pm 150$ & $4.25 \pm 0.30$ & 0.00 & 155 \\
\hline 203280 & $21: 18: 34.77$ & $+62: 35: 08.1$ & $\mathrm{~A} 8 \mathrm{~V}$ & $-11.8 \pm 1.1$ & $7800 \pm 100$ & $3.72 \pm 0.26$ & 0.00 & 210 \\
\hline 202917 & $21: 20: 49.96$ & $-53: 02: 03.1$ & G7V & $+0.1 \pm 0.2$ & $5520 \pm 60$ & $4.42 \pm 0.14$ & -0.04 & 14 \\
\hline 210302 & $22: 10: 08.78$ & $-32: 32: 54.3$ & F6V & $-16.4 \pm 0.2$ & $6460 \pm 100$ & $4.17 \pm 0.17$ & +0.18 & 13.6 \\
\hline 210418 & $22: 10: 11.99$ & $+06: 11: 52.3$ & $\mathrm{~A} 1 \mathrm{~V}$ & $-5.1 \pm 0.8$ & $8820 \pm 60$ & $4.18 \pm 0.18$ & -0.25 & 140 \\
\hline 211336 & $22: 15: 02.20$ & $+57: 02: 36.9$ & FOIV & $-5.5 \pm 0.5$ & $7400 \pm 200$ & $4.25 \pm 0.30$ & 0.20 & 91 \\
\hline 213617 & $22: 32: 35.48$ & $+20: 13: 48.1$ & F1V & $-13.1 \pm 0.7$ & $7200 \pm 200$ & $4.25 \pm 0.30$ & 0.00 & 94 \\
\hline 217782 & $23: 02: 36.38$ & $+42: 45: 28.1$ & $\mathrm{~A} 3 \mathrm{~V}$ & $+5.1 \pm 1.1$ & $8830 \pm 40$ & $3.45 \pm 0.10$ & 0.00 & 212 \\
\hline 221756 & $23: 34: 37.54$ & $+40: 14: 11.2$ & A1V & $13.5 \pm 0.3$ & $9050 \pm 60$ & $4.11 \pm 0.20$ & -0.50 & 100 \\
\hline 222368 & $23: 39: 57.04$ & $+05: 37: 34.6$ & F7V & $+5.6 \pm 0.1$ & $6130 \pm 40$ & $3.90 \pm 0.10$ & -0.16 & 6.8 \\
\hline 224392 & $23: 57: 35.08$ & $-64: 17: 53.6$ & A1V & $+9.9 \pm 2.3$ & $9400 \pm 40$ & $4.25 \pm 0.10$ & +0.10 & 195 \\
\hline
\end{tabular}




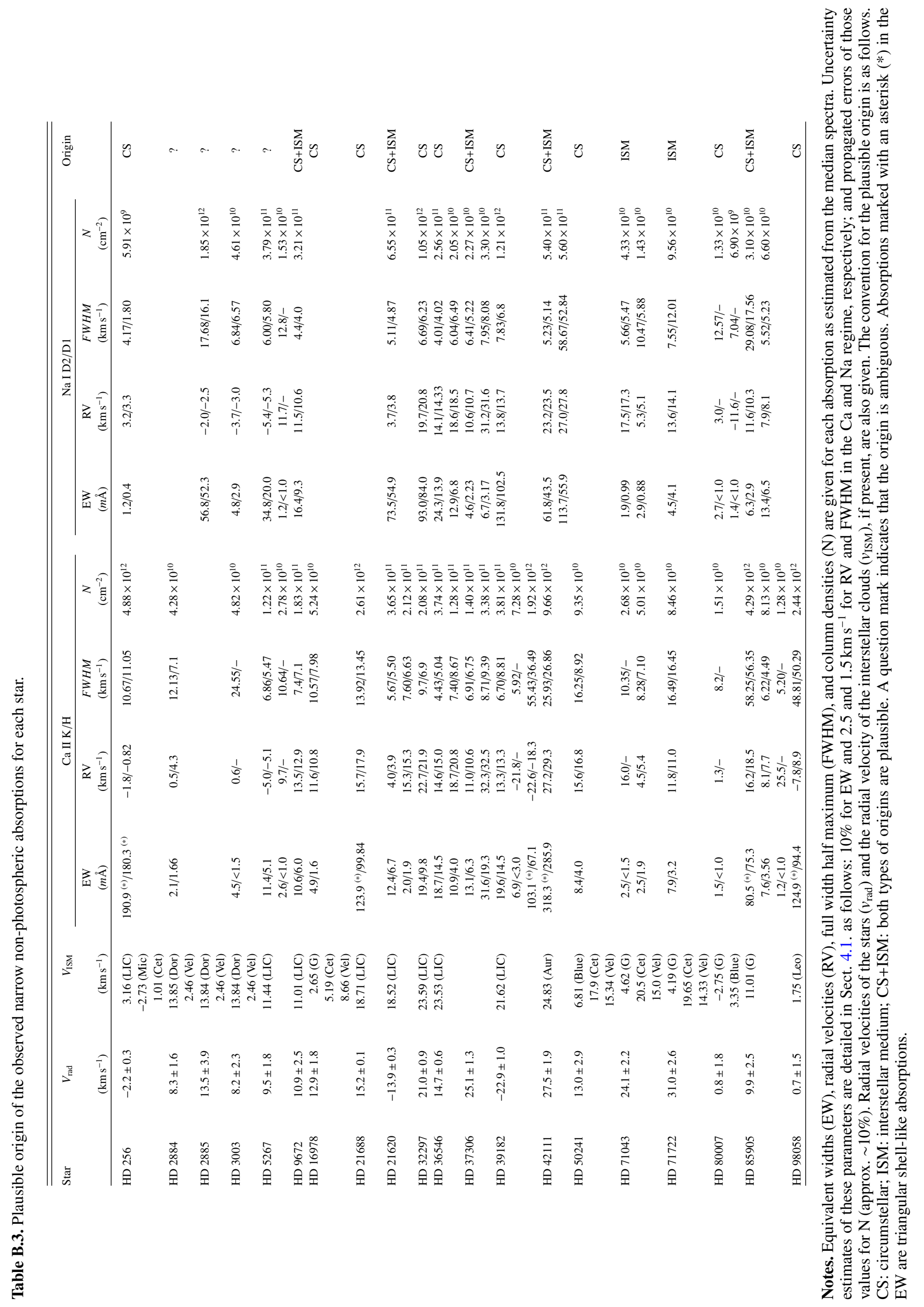




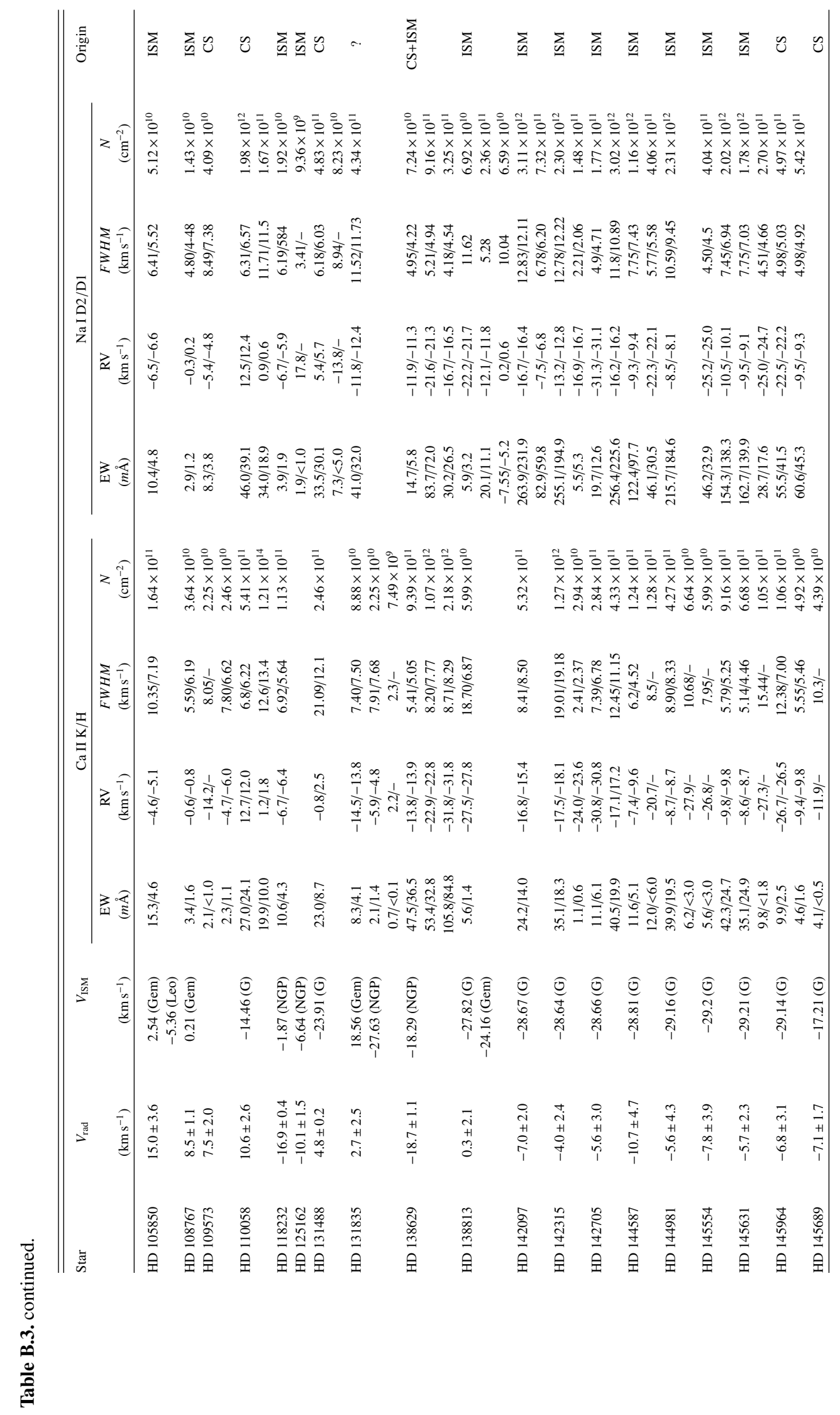




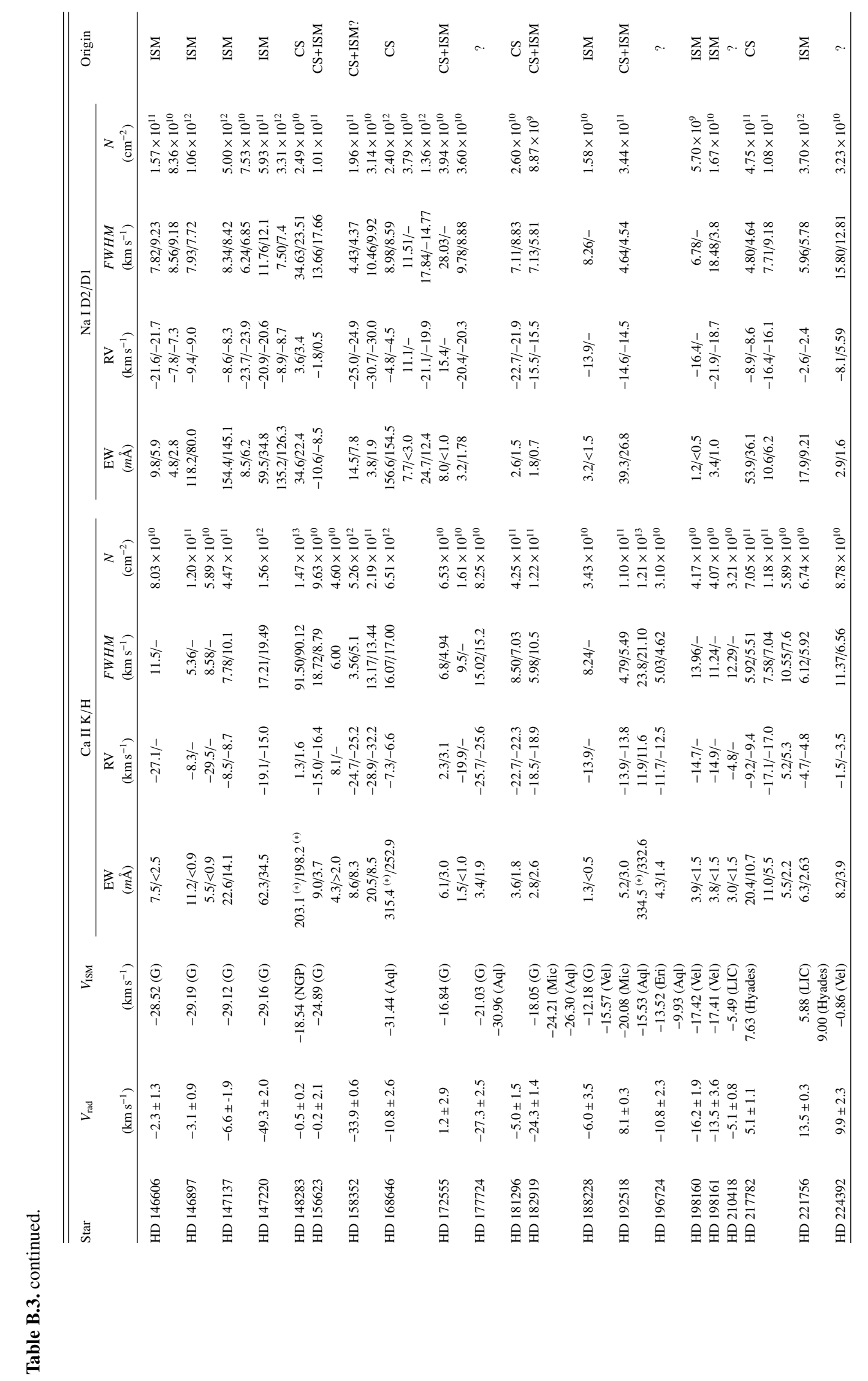

